\title{
Cytotoxic Nitrogenated Azaphilones from the Deep-Sea Derived Fungus Chaetomium globosum MP4-S01-7
}

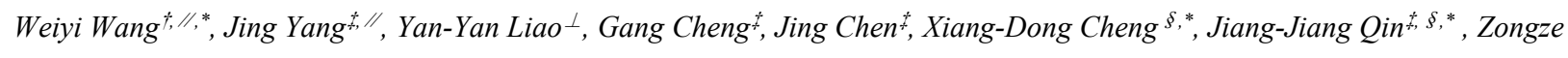
Shao ${ }^{+}$

${ }^{\dagger}$ Key Laboratory of Marine Biogenetic Resources, Third Institute of Oceanography, Ministry of Natural Resources, 184 Daxue Road, Xiamen 361005, PR China

† College of Pharmaceutical Science, Zhejiang Chinese Medical University, 548 Binwen Road, Hangzhou 310053, PR China

$\S$ Institute of Cancer and Basic Medicine, Chinese Academy of Sciences; Cancer Hospital of the University of Chinese Academy of Sciences; Zhejiang Cancer Hospital, Banshan Road 1\#, Hangzhou 310022, PR China

${ }^{\perp}$ Key Laboratory of Urban Environment and Health, Institute of Urban Environment, Chinese Academy of Sciences, 1799 Jimei Road, Xiamen 361021, PR China 


\section{Table of Content}

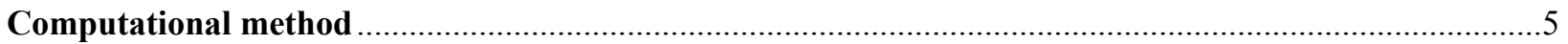

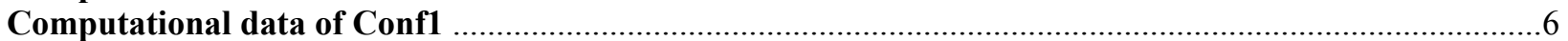

Figure S1. Optimized geometries of 8 dominant conformers of Conf1 (Conf1-1 to Conf1-8, respectively) at the B3LYP/6-31G(d) level of theory in the gas phase, and the superimposition of 8 dominant conformers........6

Table S1. Parameters of the B3LYP/6-31G(d) optimized conformers of Conf1 in the gas phase ....................6

Table S2. Conformational analysis of the B3LYP/6-31G(d) optimized conformers of Conf1 ( $\mathrm{T}=298.15 \mathrm{~K})$.....6

Table S3. Optimized Z-matrixes of Conf1-1 in the gas phase $(\AA)$ at the B3LYP/6-31G(d) level of theory........7

Table S4. Optimized Z-matrixes of Conf1-2 in the gas phase $(\AA)$ at the B3LYP/6-31G(d) level of theory........8

Table S5. Optimized Z-matrixes of Conf1-3 in the gas phase $(\AA)$ at the B3LYP/6-31G(d) level of theory........9

Table S6. Optimized Z-matrixes of Conf1-4 in the gas phase $(\AA)$ at the B3LYP/6-31G(d) level of theory......10

Table S7. Optimized Z-matrixes of Conf1-5 in the gas phase $(\AA)$ at the B3LYP/6-31G(d) level of theory......11

Table S8. Optimized Z-matrixes of Conf1-6 in the gas phase $(\AA)$ at the B3LYP/6-31G(d) level of theory......12

Table S9. Optimized Z-matrixes of Conf1-7 in the gas phase $(\AA)$ at the B3LYP/6-31G(d) level of theory......13

Table S10. Optimized Z-matrixes of Conf1-8 in the gas phase $(\AA)$ at the B3LYP/6-31G(d) level of theory ....14

Computational data of Conf2

Figure S2. Optimized geometries of 7 dominant conformers of Conf2 (Conf2-1 to Conf2-7, respectively) at the B3LYP/6-31G(d) level of theory in the gas phase, and the superimposition of 7 dominant conformers......15

Table S11. Parameters of the B3LYP/6-31G(d) optimized conformers of Conf2 in the gas phase .................15

Table S12. Conformational analysis of the B3LYP/6-31G(d) optimized conformers of Conf2 (T=298.15 K). 15

Table S13. Optimized Z-matrixes of Conf2-1 in the gas phase $(\AA)$ at the B3LYP/6-31G(d) level of theory....15

Table S14. Optimized Z-matrixes of Conf2-2 in the gas phase $(\AA)$ at the B3LYP/6-31G(d) level of theory....16

Table S15. Optimized Z-matrixes of Conf2-3 in the gas phase $(\AA)$ at the B3LYP/6-31G(d) level of theory....17

Table S16. Optimized Z-matrixes of Conf2-4 in the gas phase $(\AA)$ at the B3LYP/6-31G(d) level of theory....18

Table S17. Optimized Z-matrixes of Conf2-5 in the gas phase $(\AA)$ at the B3LYP/6-31G(d) level of theory....19

Table S18. Optimized Z-matrixes of Conf2-6 in the gas phase $(\AA)$ at the B3LYP/6-31G(d) level of theory....20

Table S19. Optimized Z-matrixes of Conf2-7 in the gas phase $(\AA)$ at the B3LYP/6-31G(d) level of theory....21

Computational data of compound 1

Table S20. Experimental and calculated ${ }^{13} \mathrm{C}-\mathrm{NMR}$ chemical shifts of Conf1 and Conf2 .............................22

Table S21. Experimental and calculated ${ }^{1} \mathrm{H}-\mathrm{NMR}$ chemical shifts of Conf1 and Conf2 ..............................23

Figure S3. Linear regression analysis between the exptl. and calcd. NMR data of Conf1 and Conf2 .............24

Figure S4. Detailed DP4+ probability (calculated at mPW1PW91/6-31+G(d,p) level) for compound 1. Isomer

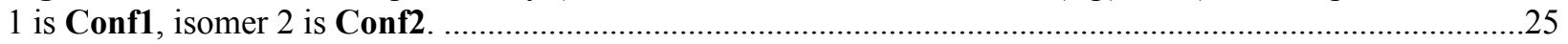

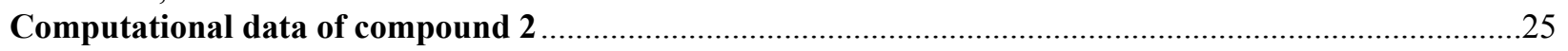

Table S22. Experimental and calculated ${ }^{13} \mathrm{C}-\mathrm{NMR}$ chemical shifts of Conf1 and Conf2 …...........................25

Table S23. Experimental and calculated 1H-NMR chemical shifts of Conf1 and Conf2 ..............................26

Figure S5. Linear regression analysis between the exptl. and calcd. NMR data of Conf1 and Conf2 .............27

Figure S6. Detailed DP4+ probability (calculated at mPW1PW91/6-31+G(d,p) level) for compound 2. Isomer

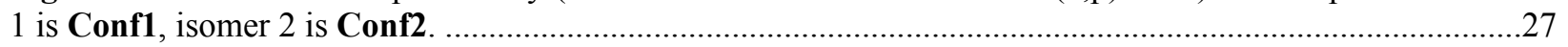

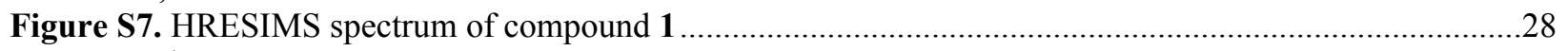

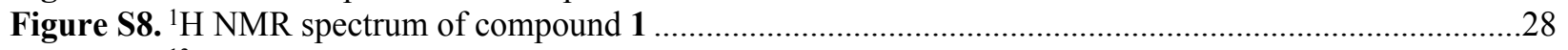

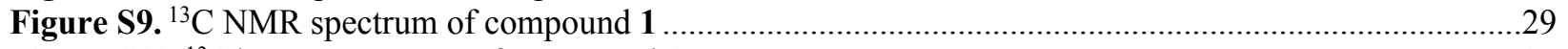

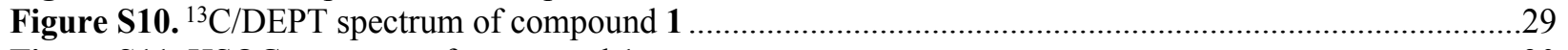

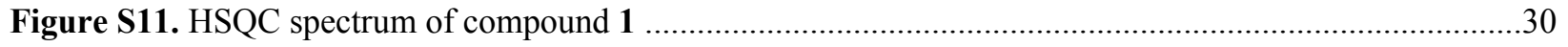

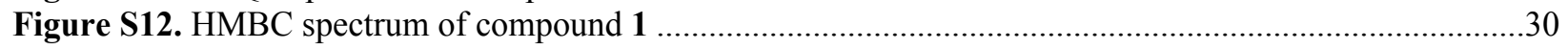

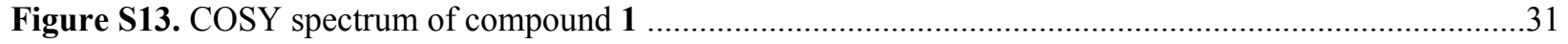

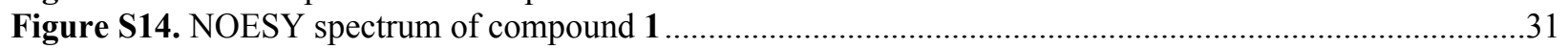

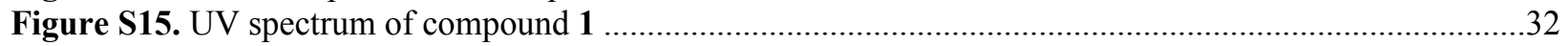

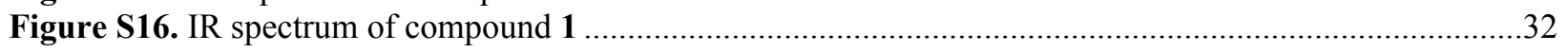

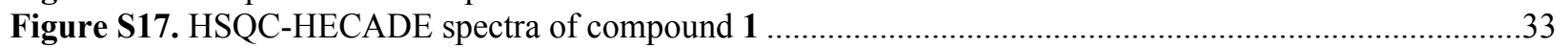

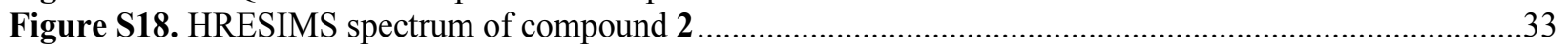

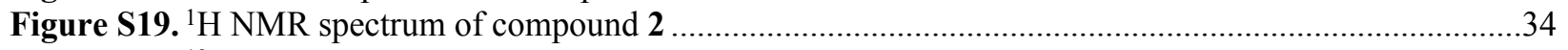

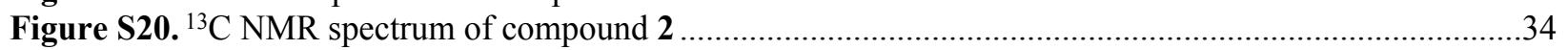




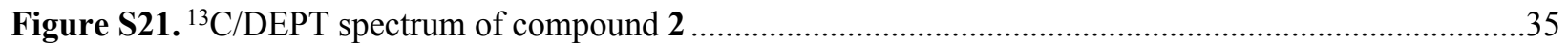

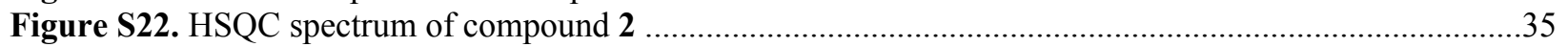

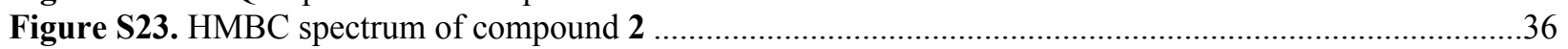

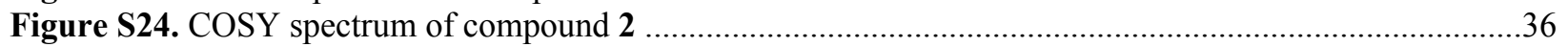

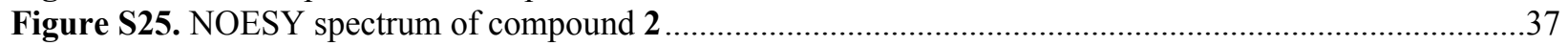

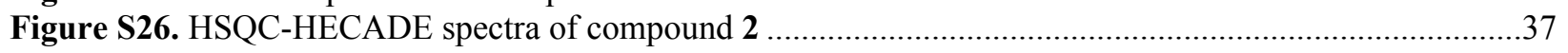

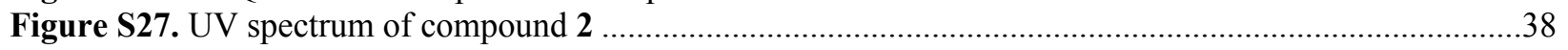

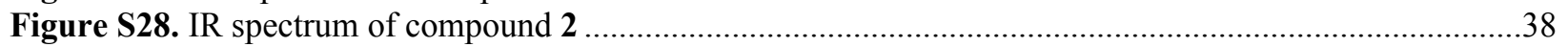

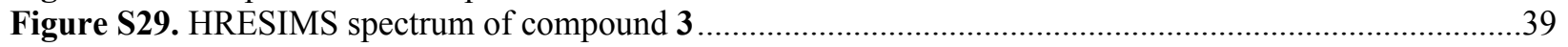

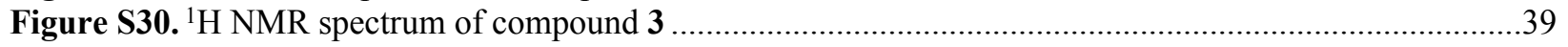

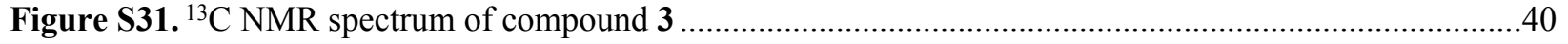

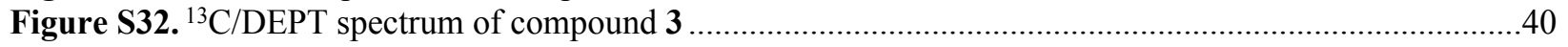

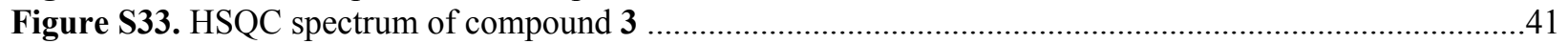

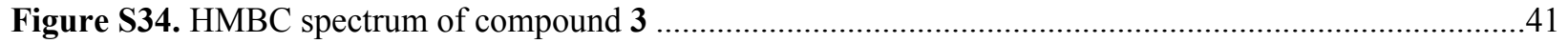

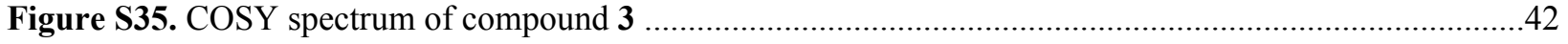

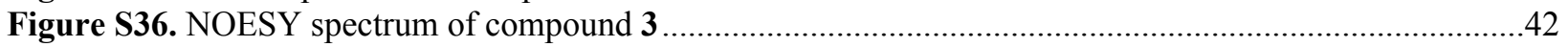

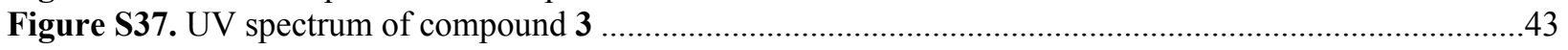

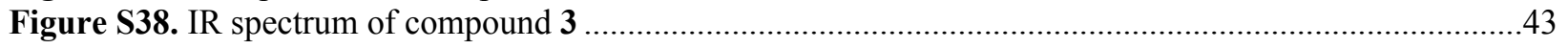

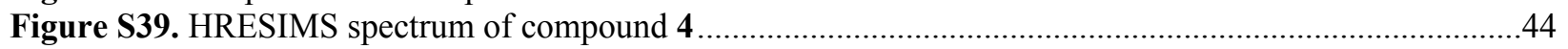

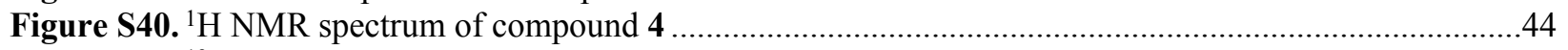

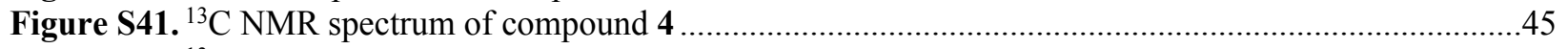

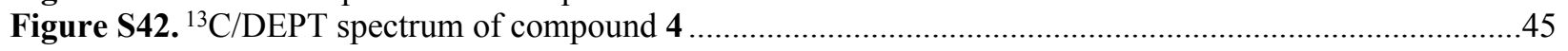

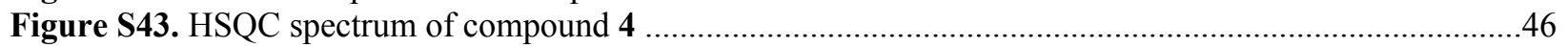

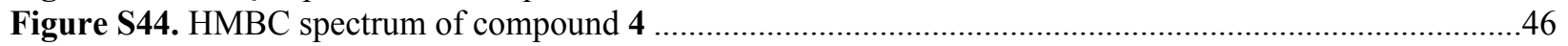

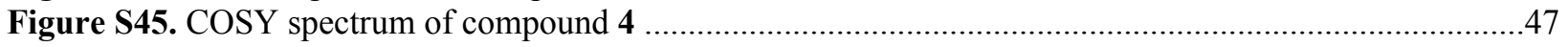

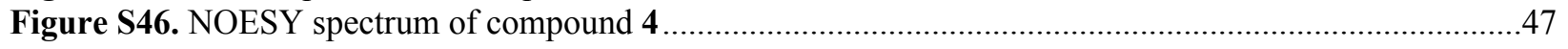

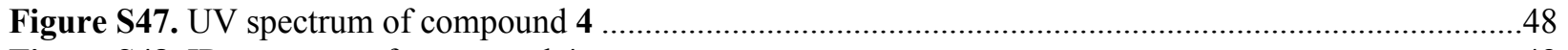

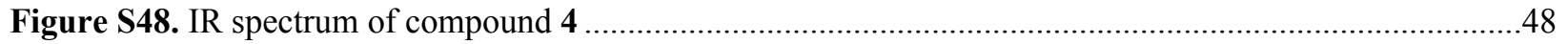

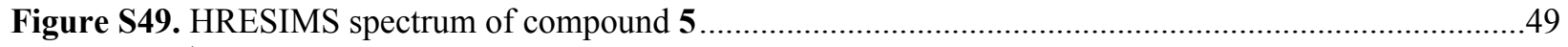

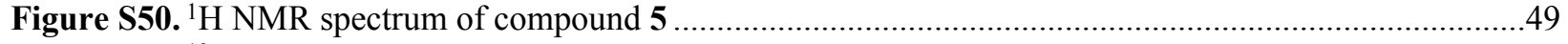

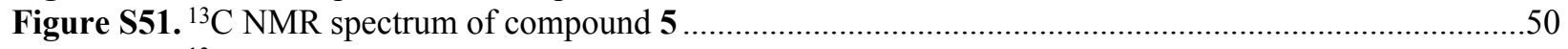

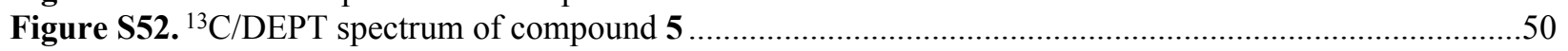

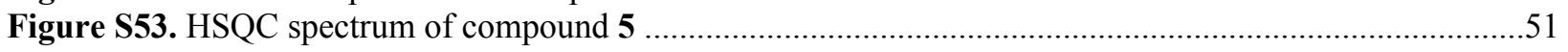

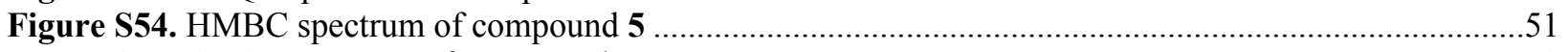

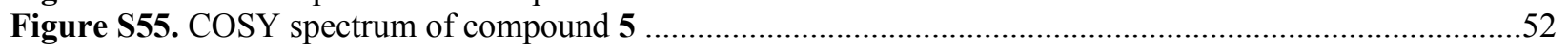

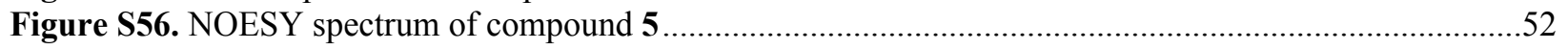

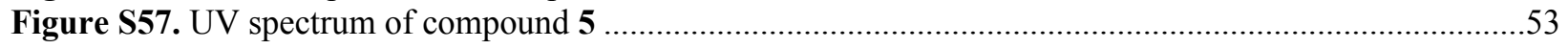

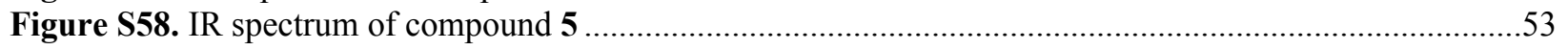

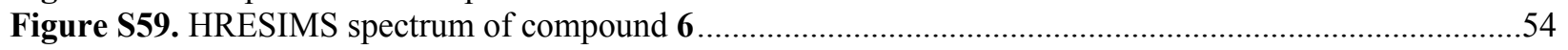

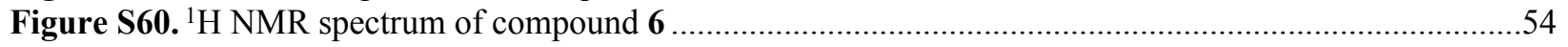

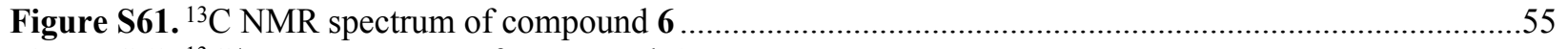

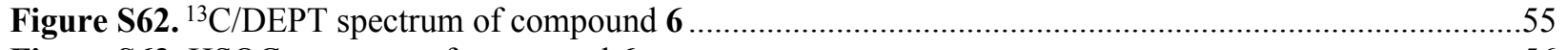

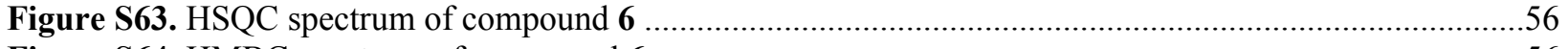

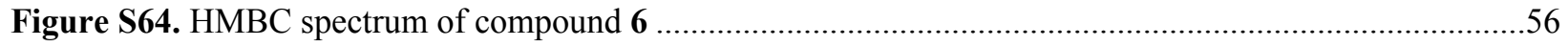

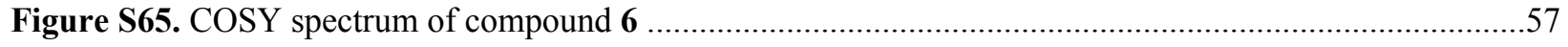

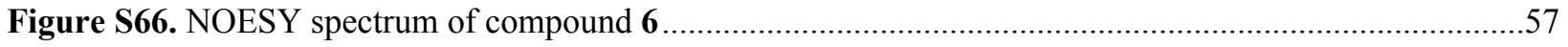

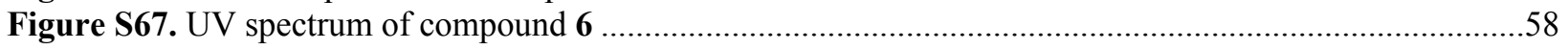

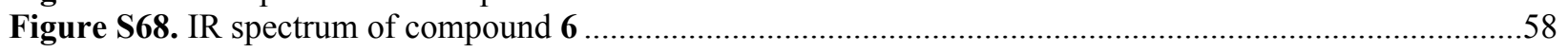

Figure S69. HRESIMS spectrum of compound 7 …...........................................................................5

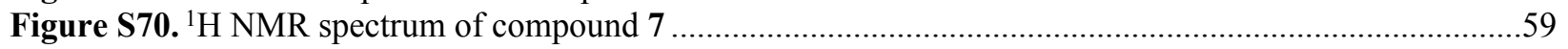

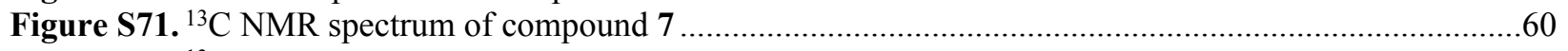

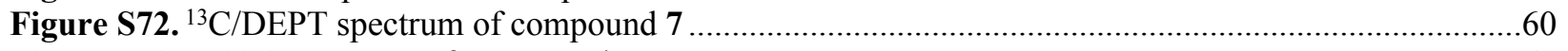

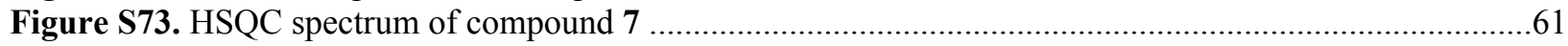




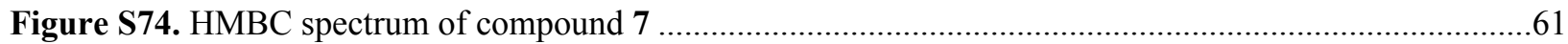

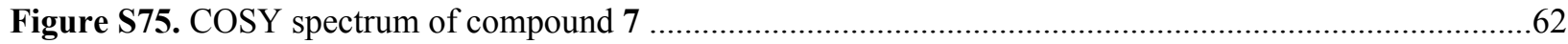

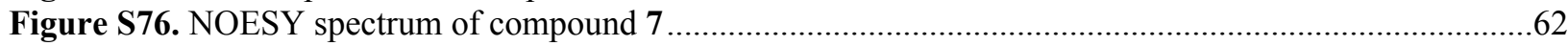

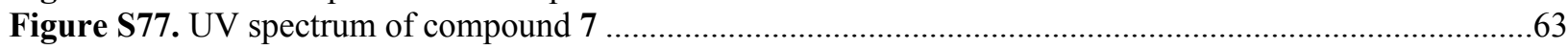

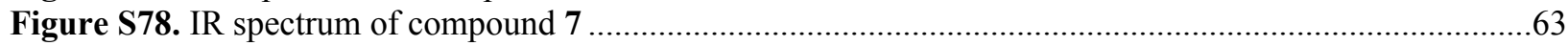

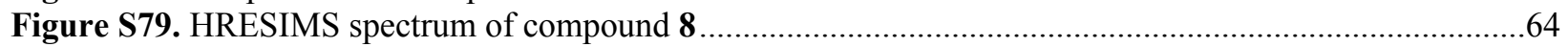

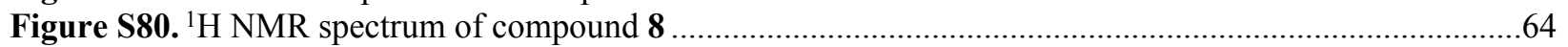

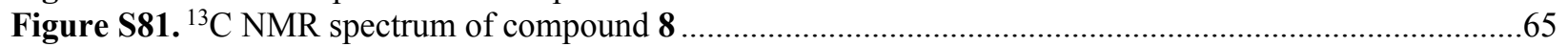

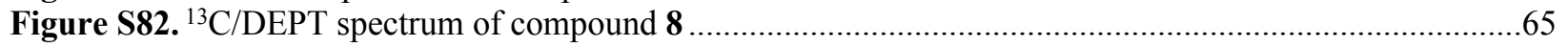

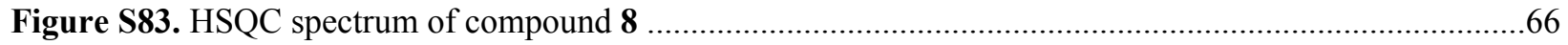

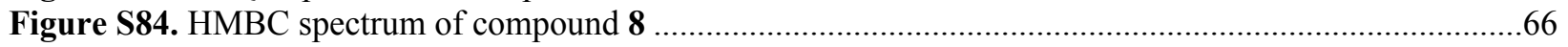

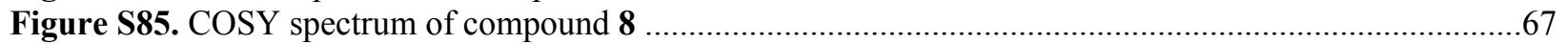

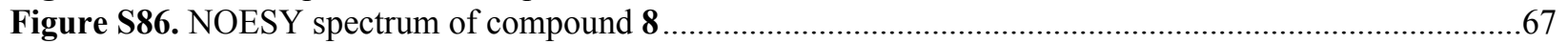

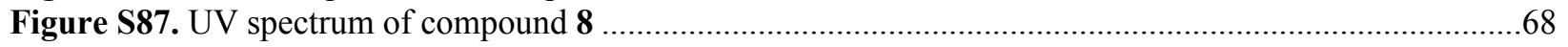

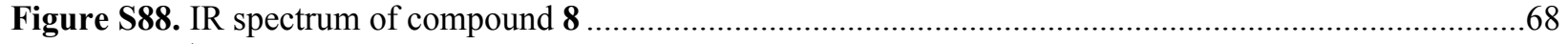

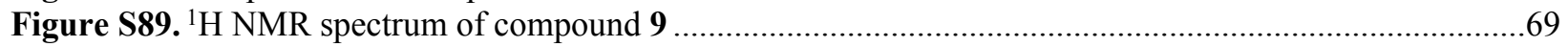

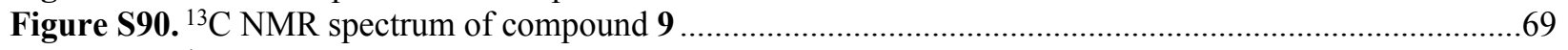

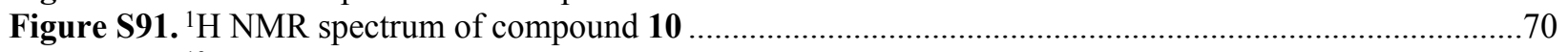

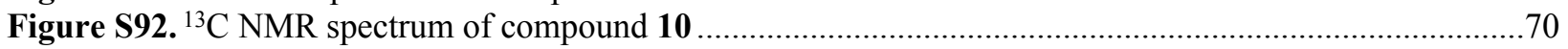

Table S24. NMR data for compound 9 in chloroform- $d$ (measured in $850 \mathrm{MHz}$ )........................................71

Figure S93. ${ }^{1} \mathrm{H}$ NMR spectrum of 2-methylbutyric acid..............................................................................

Figure S94. ${ }^{13} \mathrm{C}$ NMR spectrum of 2-methylbutyric acid ........................................................................72

Figure S95. Optical rotation of $(S)-(+)-2$-methylbutyric acid at the concentration of $1 \mathrm{mg} / \mathrm{ml}$ in chloroform. .73

Table S25. NMR data for 2-methylbutyric acid in chloroform- $d$ (measured in $850 \mathrm{MHz}$ ) ............................73

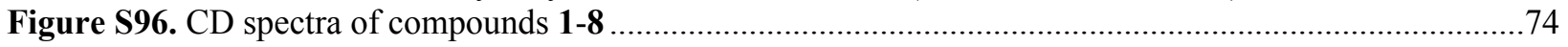

Figure S97. HRESIMS spectrum of (S)-MTPA ester of 1 (1a) …................................................................

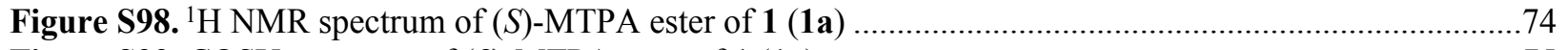

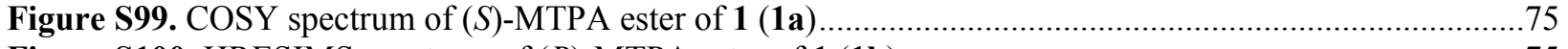

Figure S100. HRESIMS spectrum of $(R)$-MTPA ester of 1 (1b) ...............................................................

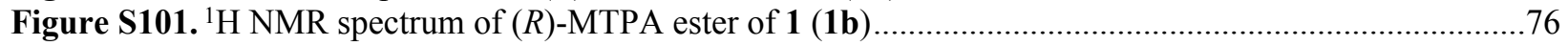

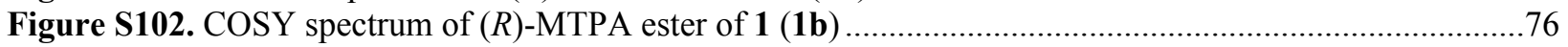

Figure S103. MTPA ester analysis (value was $\Delta \delta_{H}=\delta_{S}-\delta_{R}$ ) of compound $\mathbf{1}$ at C-4'..................................77

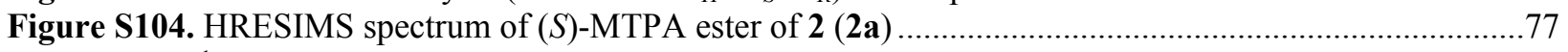

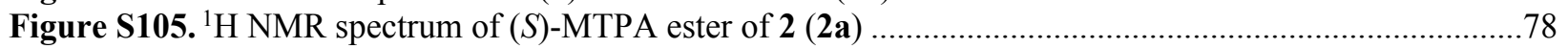

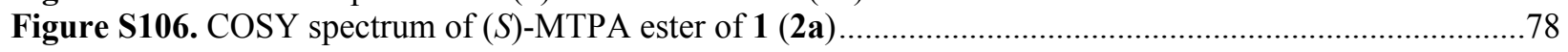

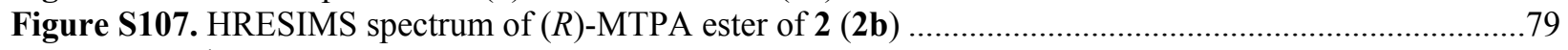

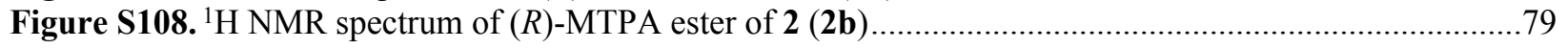

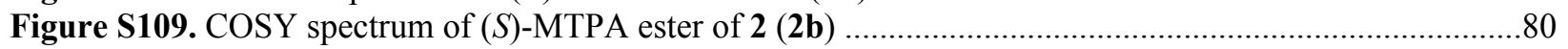

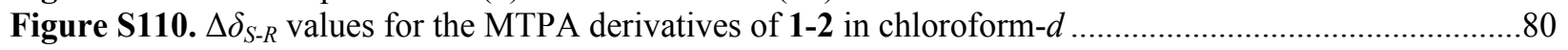

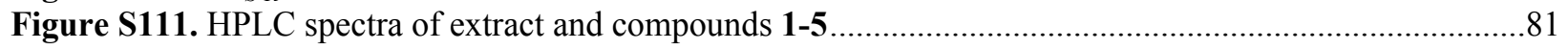

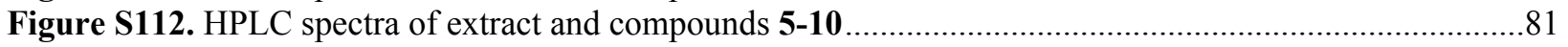

Figure S113. Preparative HPLC spectrum of $(S)$-2-methylbutyric acid from the reaction mixture of chromium trioxide oxidation of compound 1 


\section{Computational method}

The conformational search was carried out using conformer rotamer ensemble sampling tool (crest) ${ }^{1}$. The conformers within an energy window of $4 \mathrm{kcal} / \mathrm{mol}$ were optimized with DFT calculations at B3LYP/6-31G(d) level of theory with Grimme's D3 dispersion correction ("EmpiricalDispersion=GD3BJ" key words in input files) using Gaussian 09 program. ${ }^{2}$ Frequency analysis of all optimized conformations was undertaken at the same level of theory to ensure they were true local minima on the potential energy surface. Then, energies of all optimized conformations were evaluated by B3LYP/def2-TZVP with D3 dispersion correction. Gibbs free energies of each conformers were calculated by adding "Thermal correction to Gibbs Free Energy" obtained by frequency analysis to electronic energies obtained at B3LYP/def2-TZVP. Room-temperature (298.15 K) equilibrium populations were calculated according to Boltzmann distribution law. Those conformers accounting for over $99 \%$ population were subjected to subsequent calculations.

NMR shielding constants were calculated with the GIAO method at mPW1PW91-SCRF/6-31+G(d,p) level with IEFPCM solvent model in chloroform solvent. The shielding constants obtained were converted into chemical shifts by referencing to TMS at $0 \mathrm{ppm}\left(\delta_{\text {cal }}=\sigma_{\mathrm{TMS}}-\sigma_{\text {cal }}\right)$, where the $\sigma \mathrm{TMS}$ was the shielding constant of TMS calculated at the same level ${ }^{3}$. The DP4+ probabilities of each possible candidate were calculated with the EXCEL spreadsheet provided by Sarotti et $a l^{4}$.

\section{References}

(1) Grimme, S. J. Chem. Theory Comput. 2019, 15, 2847-2862.

(2) Frisch, M. J.; Trucks, G. W.; Schlegel, H. B.; Scuseria, G. E.; Robb, M. A.; Cheeseman, J. R.; Scalmani, G.; Barone, V.; Mennucci, B.; Petersson, G. A.; Nakatsuji, H.; Caricato, M.; Li, X.; Hratchian, H. P.; Izmaylov, A. F.; Bloino, J.; Zheng, G.; Sonnenberg, J. L.; Hada, M.; Ehara, M.; Toyota, K.; Fukuda, R.; Hasegawa, J.; Ishida, M.; Nakajima, T.; Honda, Y.; Kitao, O.; Nakai, H.; Vreven, T.; Montgomery, J. A.; Jr., J. E. P.; Ogliaro, F.; Bearpark, M.; Heyd, J. J.; Brothers, E.; Kudin, K. N.; Staroverov, V. N.; Keith, T.; Kobayashi, R.; Normand, J.; Raghavachari, K.; Rendell, A.; Burant, J. C.; Iyengar, S. S.; Tomasi, J.; Cossi, M.; Rega, N.; Millam, J. M.; Klene, M.; Knox, J. E.; Cross, J. B.; Bakken, V.; Adamo, C.; Jaramillo, J.; Gomperts, R.; Stratmann, R. E.; Yazyev, O.; Austin, A. J.; Cammi, R.; Pomelli, C.; Ochterski, J. W.; Martin, R. L.; Morokuma, K.; Zakrzewski, V. G.; Voth, G. A.; Salvador, P.; Dannenberg, J. J.; Dapprich, S.; Daniels, A. D.; Farkas, O.; Foresman, J. B.; Ortiz, J. V.; Cioslowski, J.; Fox, D. J. Gaussian 09; Gaussian, Inc., Wallingford CT 2010.

(3) Willoughby, P. H.; Jansma, M. J.; Hoye, T. R. Nat. Protoc. 2014, 9, 643-660.

(4) Grimblat, N.; Zanardi, M. M.; Sarotti, A. M. J. Org. Chem. 2015, 80, 12526-12534. 


\section{Computational data of Conf1}

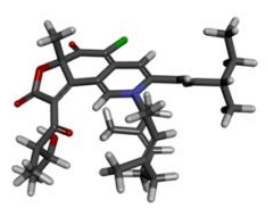

Conf1-1

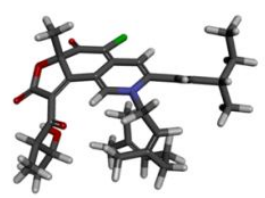

Conf1-4

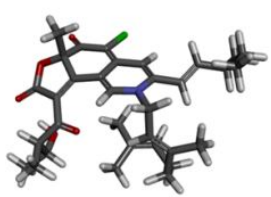

Conf1-7

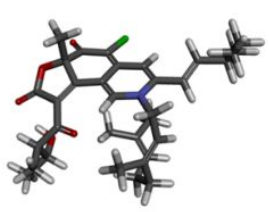

Conf1-2

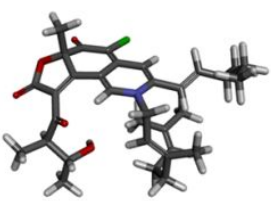

Conf1-5

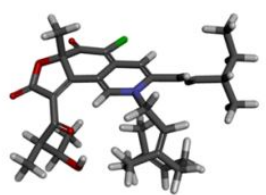

Conf1-8

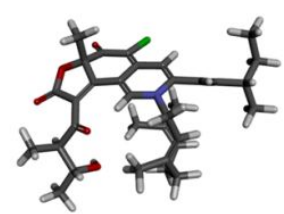

Conf1-3

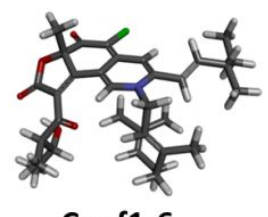

Conf1-6

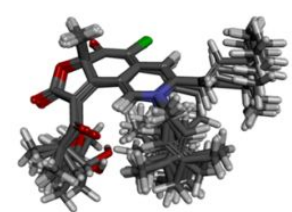

Superimposition of 8 conformers

Figure S1. Optimized geometries of 8 dominant conformers of Conf1 (Conf1-1 to Conf1-8, respectively) at the B3LYP/6-31G(d) level of theory in the gas phase, and the superimposition of 8 dominant conformers.

Table S1. Parameters of the B3LYP/6-31G(d) optimized conformers of Conf1 in the gas phase

\begin{tabular}{|c|c|c|c|}
\hline Conformers & $\mathrm{E}^{a}($ Hartree $)$ & $\mathrm{C}^{b}($ Hartree $)$ & $\mathrm{G}^{c}(\mathrm{kcal} / \mathrm{mol})$ \\
\hline Conf1-1 & -2174.708685 & 0.618219 & -1364241.767 \\
\hline Conf1-2 & -2174.707232 & 0.617148 & -1364241.528 \\
\hline Conf1-3 & -2174.709139 & 0.619419 & -1364241.299 \\
\hline Conf1-4 & -2174.70698 & 0.617789 & -1364240.967 \\
\hline Conf1-5 & -2174.705077 & 0.616506 & -1364240.579 \\
\hline Conf1-6 & -2174.705169 & 0.617273 & -1364240.155 \\
\hline Conf1-8 & -2174.705647 & 0.617962 & -1364240.023 \\
\hline Conf1-8 & -2174.706376 & 0.619223 & -1364239.689 \\
\hline
\end{tabular}

${ }^{a}$ Electronic energy obtained at B3LYP/ def2-TZVP level of theory; ${ }^{b}$ Thermal correction to Gibbs free energy obtained at B3LYP/6-31G(d) level of theory; ${ }^{C}$ Gibbs free energy $(\mathrm{E}+\mathrm{C})$.

Table S2. Conformational analysis of the B3LYP/6-31G(d) optimized conformers of Conf1 (T=298.15 K)

\begin{tabular}{|c|c|c|}
\hline Conformers & $\Delta \mathrm{G}(\mathrm{kcal} / \mathrm{mol})^{a}$ & Population $^{b}$ \\
\hline Conf1-1 & 0 & $37.6 \%$ \\
\hline Conf1-2 & 0.23949165 & $25.1 \%$ \\
\hline Conf1-3 & 0.468221675 & $17.0 \%$ \\
\hline Conf1-4 & 0.800188 & $9.7 \%$ \\
\hline Conf1-5 & 1.188805025 & $5.0 \%$ \\
\hline Conf1-6 & 1.61281305 & $2.5 \%$ \\
\hline
\end{tabular}




\begin{tabular}{|c|c|c|}
\hline Conf1-7 & 1.74478885 & $2.0 \%$ \\
\hline Conf1-8 & 2.07871925 & $1.1 \%$ \\
\hline
\end{tabular}

${ }^{a}$ The relative Gibbs free energy; ${ }^{b}$ The Boltzmann distribution of each conformer.

Table S3. Optimized Z-matrixes of Conf1-1 in the gas phase $(\AA)$ at the B3LYP/6-31G(d) level of theory.

\begin{tabular}{|c|c|c|c|c|c|c|c|}
\hline $\mathrm{C}$ & -1.66778 & -1.7509 & -2.3792 & $\mathrm{C}$ & -4.47849 & -0.0546 & -0.10204 \\
\hline $\mathrm{C}$ & -1.81479 & -2.7401 & -1.26057 & $\mathrm{C}$ & -5.91151 & -0.49218 & -0.02852 \\
\hline $\mathrm{C}$ & -0.91492 & -3.65898 & -0.88186 & $\mathrm{C}$ & -6.03912 & -1.68865 & 0.93146 \\
\hline $\mathrm{C}$ & 0.39002 & -3.94527 & -1.57573 & $\mathrm{H}$ & -7.0811 & -2.02033 & 1.00052 \\
\hline $\mathrm{H}$ & 0.44586 & -5.00864 & -1.84237 & $\mathrm{H}$ & -5.43015 & -2.53417 & 0.5942 \\
\hline $\mathrm{H}$ & 1.23745 & -3.73348 & -0.91437 & $\mathrm{H}$ & -5.70506 & -1.41535 & 1.93978 \\
\hline $\mathrm{H}$ & 0.55272 & -3.35848 & -2.48075 & $\mathrm{C}$ & -6.81652 & 0.67506 & 0.4216 \\
\hline $\mathrm{C}$ & -1.15321 & -4.44862 & 0.38482 & $\mathrm{C}$ & -6.8099 & 1.86393 & -0.54104 \\
\hline $\mathrm{C}$ & -0.5209 & -3.75491 & 1.62075 & $\mathrm{H}$ & -7.4859 & 2.65327 & -0.19629 \\
\hline $\mathrm{C}$ & -1.27888 & -2.53607 & 2.06688 & $\mathrm{H}$ & -7.13359 & 1.55966 & -1.5439 \\
\hline $\mathrm{C}$ & -0.82196 & -1.27987 & 2.17226 & $\mathrm{H}$ & -5.80803 & 2.29647 & -0.63551 \\
\hline $\mathrm{C}$ & 0.58276 & -0.85795 & 1.81211 & $\mathrm{H}$ & -7.83909 & 0.29201 & 0.53357 \\
\hline $\mathrm{H}$ & 1.27315 & -0.95958 & 2.65829 & $\mathrm{H}$ & -6.49931 & 1.0025 & 1.42165 \\
\hline $\mathrm{H}$ & 0.99396 & -1.43588 & 0.9808 & $\mathrm{H}$ & -6.23371 & -0.80879 & -1.03084 \\
\hline $\mathrm{H}$ & 0.60902 & 0.19647 & 1.52452 & $\mathrm{H}$ & -4.04109 & 0.29492 & 0.83374 \\
\hline $\mathrm{C}$ & -1.69432 & -0.18469 & 2.73221 & $\mathrm{H}$ & -4.11503 & -0.48768 & -2.1248 \\
\hline $\mathrm{H}$ & -1.25104 & 0.23834 & 3.6442 & $\mathrm{H}$ & -2.70937 & 2.10392 & -0.0783 \\
\hline $\mathrm{H}$ & -1.78704 & 0.64908 & 2.02565 & $\mathrm{Cl}$ & -1.36624 & 3.9114 & 1.28655 \\
\hline $\mathrm{H}$ & -2.69751 & -0.54751 & 2.98097 & $\mathrm{C}$ & 1.97946 & 3.69351 & -1.96272 \\
\hline $\mathrm{H}$ & -2.31175 & -2.72856 & 2.36555 & $\mathrm{H}$ & 0.95695 & 3.6698 & -2.34773 \\
\hline $\mathrm{H}$ & 0.52649 & -3.51354 & 1.41174 & $\mathrm{H}$ & 2.25313 & 4.72593 & -1.73097 \\
\hline $\mathrm{H}$ & -0.50819 & -4.48488 & 2.44315 & $\mathrm{H}$ & 2.66191 & 3.29621 & -2.71992 \\
\hline $\mathrm{H}$ & -2.22875 & -4.56872 & 0.56247 & $\mathrm{C}$ & 3.1906 & -0.75235 & -0.93575 \\
\hline $\mathrm{H}$ & -0.72901 & -5.45545 & 0.28344 & $\mathrm{O}$ & 2.38857 & -1.44222 & -1.56819 \\
\hline $\mathrm{H}$ & -2.71391 & -2.62015 & -0.66147 & $\mathrm{C}$ & 4.43282 & -1.4077 & -0.34478 \\
\hline $\mathrm{N}$ & -1.34952 & -0.39634 & -1.84849 & $\mathrm{C}$ & 4.75606 & -2.70713 & -1.09019 \\
\hline $\mathrm{C}$ & -0.04316 & -0.04176 & -1.76716 & $\mathrm{H}$ & 4.76109 & -2.54452 & -2.17177 \\
\hline $\mathrm{C}$ & 0.39953 & 1.076 & -1.10145 & $\mathrm{H}$ & 5.74028 & -3.08175 & -0.79741 \\
\hline $\mathrm{C}$ & 1.79653 & 1.40675 & -0.92493 & $\mathrm{H}$ & 4.01205 & -3.48226 & -0.88071 \\
\hline $\mathrm{C}$ & 2.95914 & 0.69663 & -0.76687 & $\mathrm{C}$ & 4.21789 & -1.65098 & 1.17755 \\
\hline $\mathrm{C}$ & 3.98535 & 1.65038 & -0.26295 & $\mathrm{C}$ & 5.44504 & -2.26623 & 1.84972 \\
\hline $\mathrm{O}$ & 5.12118 & 1.44504 & 0.11944 & $\mathrm{H}$ & 6.32728 & -1.63303 & 1.69262 \\
\hline $\mathrm{O}$ & 3.45537 & 2.89995 & -0.24078 & $\mathrm{H}$ & 5.26212 & -2.3389 & 2.92593 \\
\hline $\mathrm{C}$ & 2.09538 & 2.85878 & -0.67418 & $\mathrm{H}$ & 5.66893 & -3.267 & 1.46798 \\
\hline $\mathrm{C}$ & 1.1698 & 3.51163 & 0.38999 & $\mathrm{O}$ & 3.82336 & -0.46274 & 1.85257 \\
\hline $\mathrm{O}$ & 1.59012 & 4.41796 & 1.08873 & $\mathrm{H}$ & 4.51139 & 0.20579 & 1.68381 \\
\hline $\mathrm{C}$ & -0.19047 & 3.03265 & 0.32305 & $\mathrm{H}$ & 3.36433 & -2.33171 & 1.29004 \\
\hline $\mathrm{C}$ & -0.5744 & 1.93174 & -0.42419 & $\mathrm{H}$ & 5.26346 & -0.70145 & -0.43963 \\
\hline $\mathrm{C}$ & -1.93615 & 1.50137 & -0.53335 & $\mathrm{H}$ & 0.67125 & -0.71037 & -2.22203 \\
\hline
\end{tabular}




\begin{tabular}{|l|r|r|r|r|r|r|r|}
\hline $\mathrm{C}$ & -2.31132 & 0.36228 & -1.18778 & $\mathrm{H}$ & -2.5785 & -1.67233 & -2.97721 \\
\hline $\mathrm{C}$ & -3.70666 & -0.09376 & -1.19645 & $\mathrm{H}$ & -0.85746 & -2.01113 & -3.05894 \\
\hline
\end{tabular}

Table S4. Optimized Z-matrixes of Conf1-2 in the gas phase $(\AA)$ at the B3LYP/6-31G(d) level of theory.

\begin{tabular}{|c|c|c|c|c|c|c|c|}
\hline $\mathrm{C}$ & -1.94622 & -2.00227 & -1.59423 & $\mathrm{C}$ & -4.94975 & 0.37158 & -0.15962 \\
\hline $\mathrm{C}$ & -1.66063 & -3.0232 & -0.53385 & $\mathrm{C}$ & -6.3404 & -0.11286 & 0.13158 \\
\hline $\mathrm{C}$ & -0.62048 & -3.86884 & -0.49231 & $\mathrm{C}$ & -6.92211 & 0.64989 & 1.33503 \\
\hline $\mathrm{C}$ & 0.40932 & -4.043 & -1.57589 & $\mathrm{H}$ & -7.93167 & 0.29177 & 1.56512 \\
\hline $\mathrm{H}$ & 0.2015 & -3.46781 & -2.4807 & $\mathrm{H}$ & -6.29904 & 0.52012 & 2.22571 \\
\hline $\mathrm{H}$ & 0.47997 & -5.10137 & -1.85745 & $\mathrm{H}$ & -6.98737 & 1.72382 & 1.12248 \\
\hline $\mathrm{H}$ & 1.39818 & -3.72892 & -1.22434 & $\mathrm{C}$ & -7.24758 & 0.03776 & -1.10881 \\
\hline $\mathrm{C}$ & -0.37513 & -4.67551 & 0.76379 & $\mathrm{C}$ & -6.795 & -0.80024 & -2.3059 \\
\hline $\mathrm{C}$ & 0.43899 & -3.87083 & 1.81307 & $\mathrm{H}$ & -7.48257 & -0.68591 & -3.15046 \\
\hline $\mathrm{C}$ & -0.37159 & -2.79063 & 2.47321 & $\mathrm{H}$ & -6.75745 & -1.86576 & -2.0474 \\
\hline $\mathrm{C}$ & -0.09013 & -1.48266 & 2.56462 & $\mathrm{H}$ & -5.79506 & -0.50592 & -2.64321 \\
\hline $\mathrm{C}$ & 1.14965 & -0.84072 & 1.9934 & $\mathrm{H}$ & -8.26834 & -0.24648 & -0.82225 \\
\hline $\mathrm{H}$ & 1.965 & -0.80623 & 2.72652 & $\mathrm{H}$ & -7.29162 & 1.09915 & -1.39058 \\
\hline $\mathrm{H}$ & 1.52311 & -1.36327 & 1.11131 & $\mathrm{H}$ & -6.28814 & -1.18088 & 0.38703 \\
\hline $\mathrm{H}$ & 0.95431 & 0.1967 & 1.7065 & $\mathrm{H}$ & -4.85497 & 1.43046 & -0.40555 \\
\hline $\mathrm{C}$ & -1.0071 & -0.54528 & 3.30913 & $\mathrm{H}$ & -3.95356 & -1.45667 & 0.06179 \\
\hline $\mathrm{H}$ & -1.86809 & -1.06738 & 3.7392 & $\mathrm{H}$ & -2.78626 & 1.88267 & 0.74863 \\
\hline $\mathrm{H}$ & -0.47186 & -0.03863 & 4.12374 & $\mathrm{Cl}$ & -1.40853 & 3.95646 & 1.57793 \\
\hline $\mathrm{H}$ & -1.37808 & 0.24623 & 2.64513 & $\mathrm{C}$ & 1.30111 & 3.60079 & -2.18194 \\
\hline $\mathrm{H}$ & -1.28979 & -3.1444 & 2.94775 & $\mathrm{H}$ & 0.23133 & 3.46726 & -2.36017 \\
\hline $\mathrm{H}$ & 1.33995 & -3.46167 & 1.34383 & $\mathrm{H}$ & 1.52261 & 4.66799 & -2.10069 \\
\hline $\mathrm{H}$ & 0.78585 & -4.57956 & 2.57952 & $\mathrm{H}$ & 1.86498 & 3.17343 & -3.01642 \\
\hline $\mathrm{H}$ & -1.32778 & -4.97626 & 1.21692 & $\mathrm{C}$ & 3.07497 & -0.60862 & -1.11071 \\
\hline $\mathrm{H}$ & 0.16699 & -5.59631 & 0.51762 & $\mathrm{O}$ & 2.21673 & -1.41499 & -1.47653 \\
\hline $\mathrm{H}$ & -2.31676 & -2.98403 & 0.33341 & $\mathrm{C}$ & 4.48564 & -1.10726 & -0.82205 \\
\hline $\mathrm{N}$ & -1.59903 & -0.63925 & -1.10993 & $\mathrm{C}$ & 4.75446 & -2.41199 & -1.58015 \\
\hline $\mathrm{C}$ & -0.32419 & -0.21503 & -1.27739 & $\mathrm{H}$ & 4.17372 & -3.24151 & -1.16429 \\
\hline $\mathrm{C}$ & 0.13967 & 0.99198 & -0.80966 & $\mathrm{H}$ & 4.47728 & -2.31529 & -2.63392 \\
\hline $\mathrm{C}$ & 1.51248 & 1.42815 & -0.93432 & $\mathrm{H}$ & 5.81506 & -2.67189 & -1.53054 \\
\hline $\mathrm{C}$ & 2.74487 & 0.82392 & -0.97762 & $\mathrm{C}$ & 4.66878 & -1.28289 & 0.71392 \\
\hline $\mathrm{C}$ & 3.75899 & 1.8923 & -0.76097 & $\mathrm{C}$ & 6.08731 & -1.71383 & 1.08572 \\
\hline $\mathrm{O}$ & 4.96233 & 1.80773 & -0.60614 & $\mathrm{H}$ & 6.81942 & -0.98854 & 0.7091 \\
\hline $\mathrm{O}$ & 3.1328 & 3.09562 & -0.71298 & $\mathrm{H}$ & 6.17367 & -1.75416 & 2.17564 \\
\hline $\mathrm{C}$ & 1.72553 & 2.91573 & -0.86842 & $\mathrm{H}$ & 6.3402 & -2.69855 & 0.68105 \\
\hline $\mathrm{C}$ & 0.95779 & 3.61427 & 0.28553 & $\mathrm{O}$ & 4.29874 & -0.11029 & 1.4279 \\
\hline $\mathrm{O}$ & 1.42175 & 4.61168 & 0.81128 & $\mathrm{H}$ & 4.83843 & 0.62106 & 1.07707 \\
\hline $\mathrm{C}$ & -0.3517 & 3.04908 & 0.50991 & $\mathrm{H}$ & 3.95741 & -2.0493 & 1.04714 \\
\hline $\mathrm{C}$ & -0.76981 & 1.85336 & -0.05046 & $\mathrm{H}$ & 5.19062 & -0.33421 & -1.14201 \\
\hline $\mathrm{C}$ & -2.08769 & 1.32471 & 0.14156 & $\mathrm{H}$ & 0.34771 & -0.8848 & -1.79208 \\
\hline
\end{tabular}




\begin{tabular}{|l|l|l|l|l|l|l|r|}
\hline $\mathrm{C}$ & -2.49601 & 0.12465 & -0.36989 & $\mathrm{H}$ & -2.99895 & -1.98192 & -1.88613 \\
\hline $\mathrm{C}$ & -3.85059 & -0.39497 & -0.14788 & $\mathrm{H}$ & -1.36116 & -2.17148 & -2.49798 \\
\hline
\end{tabular}

Table S5. Optimized Z-matrixes of Conf1-3 in the gas phase $(\AA)$ at the B3LYP/6-31G(d) level of theory.

\begin{tabular}{|c|c|c|c|c|c|c|c|}
\hline $\mathrm{C}$ & -1.35945 & -1.77295 & -2.38275 & $\mathrm{C}$ & -4.43369 & -0.19847 & -0.36476 \\
\hline $\mathrm{C}$ & -1.59958 & -2.74664 & -1.26597 & $\mathrm{C}$ & -5.83805 & -0.72335 & -0.41605 \\
\hline $\mathrm{C}$ & -0.68145 & -3.54015 & -0.69582 & $\mathrm{C}$ & -5.97735 & -1.91444 & 0.54944 \\
\hline $\mathrm{C}$ & 0.74709 & -3.68903 & -1.14162 & $\mathrm{H}$ & -6.99651 & -2.31602 & 0.52495 \\
\hline $\mathrm{H}$ & 1.00076 & -3.12029 & -2.03672 & $\mathrm{H}$ & -5.28307 & -2.72008 & 0.28772 \\
\hline $\mathrm{H}$ & 0.96758 & -4.74741 & -1.3345 & $\mathrm{H}$ & -5.76125 & -1.60707 & 1.57989 \\
\hline $\mathrm{H}$ & 1.42585 & -3.3585 & -0.34874 & $\mathrm{C}$ & -6.85338 & 0.3874 & -0.07257 \\
\hline $\mathrm{C}$ & -1.03658 & -4.30503 & 0.55714 & $\mathrm{C}$ & -6.83719 & 1.55796 & -1.05734 \\
\hline $\mathrm{C}$ & -0.60416 & -3.53729 & 1.83515 & $\mathrm{H}$ & -7.58705 & 2.30934 & -0.78891 \\
\hline $\mathrm{C}$ & -1.47355 & -2.34658 & 2.12928 & $\mathrm{H}$ & -7.05446 & 1.21672 & -2.07687 \\
\hline $\mathrm{C}$ & -1.10726 & -1.07222 & 2.32983 & $\mathrm{H}$ & -5.85882 & 2.05013 & -1.07644 \\
\hline $\mathrm{C}$ & 0.30968 & -0.56042 & 2.24008 & $\mathrm{H}$ & -7.85498 & -0.06071 & -0.0427 \\
\hline $\mathrm{H}$ & 0.73097 & -0.41709 & 3.24608 & $\mathrm{H}$ & -6.64898 & 0.7518 & 0.94406 \\
\hline $\mathrm{H}$ & 0.97362 & -1.22297 & 1.68194 & $\mathrm{H}$ & -6.04512 & -1.07326 & -1.43752 \\
\hline $\mathrm{H}$ & 0.3293 & 0.42391 & 1.75991 & $\mathrm{H}$ & -4.10836 & 0.18944 & 0.6005 \\
\hline $\mathrm{C}$ & -2.12176 & -0.03538 & 2.74405 & $\mathrm{H}$ & -3.85668 & -0.64608 & -2.33291 \\
\hline $\mathrm{H}$ & -1.8788 & 0.37111 & 3.73576 & $\mathrm{H}$ & -2.76022 & 2.0168 & -0.16633 \\
\hline $\mathrm{H}$ & -2.12336 & 0.82098 & 2.05965 & $\mathrm{Cl}$ & -1.59723 & 3.8233 & 1.37595 \\
\hline $\mathrm{H}$ & -3.13397 & -0.45149 & 2.79102 & $\mathrm{C}$ & 1.92302 & 3.91372 & -1.63693 \\
\hline $\mathrm{H}$ & -2.53522 & -2.58289 & 2.23347 & $\mathrm{H}$ & 0.92323 & 3.85275 & -2.07379 \\
\hline $\mathrm{H}$ & 0.4515 & -3.26073 & 1.76023 & $\mathrm{H}$ & 2.12568 & 4.94659 & -1.34262 \\
\hline $\mathrm{H}$ & -0.67895 & -4.23813 & 2.68018 & $\mathrm{H}$ & 2.66311 & 3.59334 & -2.37617 \\
\hline $\mathrm{H}$ & -2.11761 & -4.48627 & 0.60057 & $\mathrm{C}$ & 3.35861 & -0.48674 & -0.84422 \\
\hline $\mathrm{H}$ & -0.54341 & -5.28526 & 0.54948 & $\mathrm{O}$ & 2.74117 & -1.15175 & -1.67491 \\
\hline $\mathrm{H}$ & -2.59455 & -2.71144 & -0.82989 & $\mathrm{C}$ & 4.53226 & -1.1198 & -0.09699 \\
\hline $\mathrm{N}$ & -1.14936 & -0.39791 & -1.84509 & $\mathrm{C}$ & 5.75348 & -1.10547 & -1.03621 \\
\hline $\mathrm{C}$ & 0.12523 & 0.03547 & -1.69068 & $\mathrm{H}$ & 5.97147 & -0.08316 & -1.35388 \\
\hline $\mathrm{C}$ & 0.45908 & 1.16701 & -0.9841 & $\mathrm{H}$ & 6.64139 & -1.48549 & -0.5238 \\
\hline $\mathrm{C}$ & 1.82197 & 1.5726 & -0.73366 & $\mathrm{H}$ & 5.56226 & -1.72204 & -1.92134 \\
\hline $\mathrm{C}$ & 3.02044 & 0.92594 & -0.59081 & $\mathrm{C}$ & 4.13574 & -2.53213 & 0.36007 \\
\hline $\mathrm{C}$ & 3.99239 & 1.90527 & -0.02957 & $\mathrm{C}$ & 5.25146 & -3.27483 & 1.08819 \\
\hline $\mathrm{O}$ & 5.15208 & 1.75075 & 0.27872 & $\mathrm{H}$ & 5.62517 & -2.68698 & 1.93721 \\
\hline $\mathrm{O}$ & 3.36543 & 3.1111 & 0.1077 & $\mathrm{H}$ & 4.86815 & -4.22594 & 1.4699 \\
\hline $\mathrm{C}$ & 2.02936 & 3.0212 & -0.38378 & $\mathrm{H}$ & 6.09788 & -3.48492 & 0.42788 \\
\hline $\mathrm{C}$ & 1.01416 & 3.56405 & 0.65537 & $\mathrm{O}$ & 2.95989 & -2.46866 & 1.1859 \\
\hline $\mathrm{O}$ & 1.34483 & 4.43847 & 1.4385 & $\mathrm{H}$ & 3.19523 & -1.99881 & 2.00264 \\
\hline $\mathrm{C}$ & -0.31801 & 3.03567 & 0.46567 & $\mathrm{H}$ & 3.81492 & -3.10259 & -0.51526 \\
\hline $\mathrm{C}$ & -0.59888 & 1.94743 & -0.34424 & $\mathrm{H}$ & 4.77511 & -0.51421 & 0.78165 \\
\hline $\mathrm{C}$ & -1.92597 & 1.45672 & -0.56436 & $\mathrm{H}$ & 0.90641 & -0.56736 & -2.13257 \\
\hline
\end{tabular}




\begin{tabular}{|l|r|r|r|r|r|r|r|}
\hline $\mathrm{C}$ & -2.19385 & 0.30724 & -1.25278 & $\mathrm{H}$ & -2.19682 & -1.73859 & -3.08346 \\
\hline $\mathrm{C}$ & -3.55948 & -0.21409 & -1.3798 & $\mathrm{H}$ & -0.46642 & -2.01322 & -2.95873 \\
\hline
\end{tabular}

Table S6. Optimized Z-matrixes of Conf1-4 in the gas phase $(\AA)$ at the B3LYP/6-31G(d) level of theory.

\begin{tabular}{|c|c|c|c|c|c|c|c|}
\hline $\mathrm{C}$ & -1.67721 & -1.33471 & -2.67548 & $\mathrm{C}$ & -4.44423 & 0.17057 & -0.18944 \\
\hline $\mathrm{C}$ & -2.15734 & -2.39143 & -1.71711 & $\mathrm{C}$ & -5.88055 & -0.263 & -0.18667 \\
\hline $\mathrm{C}$ & -1.38381 & -3.13008 & -0.90465 & $\mathrm{C}$ & -6.03229 & -1.54272 & 0.65575 \\
\hline $\mathrm{C}$ & 0.11967 & -3.11427 & -0.90727 & $\mathrm{H}$ & -5.73222 & -1.36328 & 1.69514 \\
\hline $\mathrm{H}$ & 0.55929 & -2.60947 & -1.76811 & $\mathrm{H}$ & -7.0742 & -1.88172 & 0.65991 \\
\hline $\mathrm{H}$ & 0.50189 & -4.14253 & -0.88469 & $\mathrm{H}$ & -5.40779 & -2.35245 & 0.26323 \\
\hline $\mathrm{H}$ & 0.49753 & -2.62788 & -0.00094 & $\mathrm{C}$ & -6.79597 & 0.86092 & 0.34367 \\
\hline $\mathrm{C}$ & -2.05282 & -3.98589 & 0.14791 & $\mathrm{C}$ & -6.77269 & 2.12592 & -0.51628 \\
\hline $\mathrm{C}$ & -2.20084 & -3.23954 & 1.50524 & $\mathrm{H}$ & -5.76848 & 2.56131 & -0.55916 \\
\hline $\mathrm{C}$ & -0.94065 & -3.13833 & 2.32178 & $\mathrm{H}$ & -7.45264 & 2.88603 & -0.11777 \\
\hline $\mathrm{C}$ & -0.17019 & -2.0602 & 2.536 & $\mathrm{H}$ & -7.08143 & 1.90666 & -1.54573 \\
\hline $\mathrm{C}$ & -0.39618 & -0.71069 & 1.90471 & $\mathrm{H}$ & -7.81987 & 0.46985 & 0.40375 \\
\hline $\mathrm{H}$ & -1.21996 & -0.7035 & 1.1885 & $\mathrm{H}$ & -6.49831 & 1.10326 & 1.3735 \\
\hline $\mathrm{H}$ & -0.5968 & 0.05678 & 2.66423 & $\mathrm{H}$ & -6.17834 & -0.48708 & -1.22105 \\
\hline $\mathrm{H}$ & 0.51305 & -0.3887 & 1.38118 & $\mathrm{H}$ & -4.02513 & 0.43443 & 0.7828 \\
\hline $\mathrm{C}$ & 1.02407 & -2.12808 & 3.45493 & $\mathrm{H}$ & -4.04397 & -0.10228 & -2.23148 \\
\hline $\mathrm{H}$ & 1.18522 & -3.14132 & 3.83758 & $\mathrm{H}$ & -2.57784 & 2.21265 & 0.13278 \\
\hline $\mathrm{H}$ & 1.93534 & -1.78514 & 2.95035 & $\mathrm{Cl}$ & -1.15531 & 3.71956 & 1.7659 \\
\hline $\mathrm{H}$ & 0.87962 & -1.46096 & 4.31677 & $\mathrm{C}$ & 2.21455 & 3.93278 & -1.46875 \\
\hline $\mathrm{H}$ & -0.6458 & -4.0684 & 2.81089 & $\mathrm{H}$ & 1.19736 & 4.03995 & -1.85407 \\
\hline $\mathrm{H}$ & -2.94631 & -3.78854 & 2.09727 & $\mathrm{H}$ & 2.54162 & 4.88616 & -1.04604 \\
\hline $\mathrm{H}$ & -2.63176 & -2.25268 & 1.30593 & $\mathrm{H}$ & 2.88351 & 3.64482 & -2.28508 \\
\hline $\mathrm{H}$ & -3.05491 & -4.26713 & -0.19743 & $\mathrm{C}$ & 3.1835 & -0.65726 & -1.38108 \\
\hline $\mathrm{H}$ & -1.49133 & -4.91538 & 0.30611 & $\mathrm{O}$ & 2.43971 & -1.10613 & -2.25553 \\
\hline $\mathrm{H}$ & -3.23562 & -2.48767 & -1.62327 & $\mathrm{C}$ & 4.28727 & -1.5379 & -0.81061 \\
\hline $\mathrm{N}$ & -1.31093 & -0.07051 & -1.97345 & $\mathrm{C}$ & 4.57231 & -2.71515 & -1.74804 \\
\hline $\mathrm{C}$ & -0.00172 & 0.26872 & -1.90214 & $\mathrm{H}$ & 3.73747 & -3.42309 & -1.75945 \\
\hline $\mathrm{C}$ & 0.4822 & 1.26487 & -1.08782 & $\mathrm{H}$ & 4.71729 & -2.36671 & -2.7743 \\
\hline $\mathrm{C}$ & 1.89151 & 1.5093 & -0.88938 & $\mathrm{H}$ & 5.47612 & -3.2457 & -1.43735 \\
\hline $\mathrm{C}$ & 3.01627 & 0.72645 & -0.89406 & $\mathrm{C}$ & 3.88067 & -2.01673 & 0.61315 \\
\hline $\mathrm{C}$ & 4.07785 & 1.49117 & -0.1867 & $\mathrm{C}$ & 4.96476 & -2.86098 & 1.2807 \\
\hline $\mathrm{O}$ & 5.18314 & 1.13493 & 0.173 & $\mathrm{H}$ & 5.9074 & -2.30194 & 1.33499 \\
\hline $\mathrm{O}$ & 3.619 & 2.74195 & 0.08061 & $\mathrm{H}$ & 4.65109 & -3.10651 & 2.29978 \\
\hline $\mathrm{C}$ & 2.26443 & 2.86602 & -0.35975 & $\mathrm{H}$ & 5.15144 & -3.79487 & 0.7423 \\
\hline $\mathrm{C}$ & 1.36187 & 3.3626 & 0.80171 & $\mathrm{O}$ & 3.51576 & -0.92422 & 1.45366 \\
\hline $\mathrm{O}$ & 1.81986 & 4.10666 & 1.65251 & $\mathrm{H}$ & 4.28438 & -0.32864 & 1.50782 \\
\hline $\mathrm{C}$ & -0.02061 & 2.97096 & 0.65433 & $\mathrm{H}$ & 2.96342 & -2.6116 & 0.52178 \\
\hline $\mathrm{C}$ & -0.45179 & 2.02279 & -0.25703 & $\mathrm{H}$ & 5.18215 & -0.91728 & -0.69518 \\
\hline $\mathrm{C}$ & -1.82841 & 1.65602 & -0.41211 & $\mathrm{H}$ & 0.68807 & -0.30971 & -2.49853 \\
\hline
\end{tabular}




\begin{tabular}{|l|r|r|r|l|r|r|r|}
\hline $\mathrm{C}$ & -2.24371 & 0.6243 & -1.20532 & $\mathrm{H}$ & -2.44055 & -1.10501 & -3.42351 \\
\hline $\mathrm{C}$ & -3.64872 & 0.20934 & -1.26676 & $\mathrm{H}$ & -0.77999 & -1.64374 & -3.21426 \\
\hline
\end{tabular}

Table S7. Optimized Z-matrixes of Conf1-5 in the gas phase $(\AA)$ at the B3LYP/6-31G(d) level of theory.

\begin{tabular}{|c|c|c|c|c|c|c|c|}
\hline $\mathrm{C}$ & 1.50994 & 1.28463 & -2.11102 & $\mathrm{C}$ & 4.04278 & -1.45511 & -0.54366 \\
\hline $\mathrm{C}$ & 1.48276 & 2.38445 & -1.08845 & $\mathrm{C}$ & 5.48885 & -1.18296 & -0.24019 \\
\hline $\mathrm{C}$ & 2.5228 & 3.07679 & -0.60644 & $\mathrm{C}$ & 5.96678 & -2.0832 & 0.91143 \\
\hline C & 3.9435 & 2.95324 & -1.08975 & $\mathrm{H}$ & 7.01775 & -1.8826 & 1.1478 \\
\hline $\mathrm{H}$ & 4.07591 & 2.24969 & -1.91505 & $\mathrm{H}$ & 5.37316 & -1.9163 & 1.81589 \\
\hline $\mathrm{H}$ & 4.59701 & 2.63958 & -0.26394 & $\mathrm{H}$ & 5.88013 & -3.1427 & 0.64124 \\
\hline $\mathrm{H}$ & 4.31508 & 3.93075 & -1.42392 & $\mathrm{C}$ & 6.36493 & -1.37624 & -1.49703 \\
\hline C & 2.3122 & 4.04529 & 0.54776 & $\mathrm{C}$ & 6.03423 & -0.40149 & -2.62877 \\
\hline $\mathrm{C}$ & 1.69318 & 3.37044 & 1.79332 & $\mathrm{H}$ & 6.69305 & -0.5556 & -3.48982 \\
\hline $\mathrm{C}$ & 2.50202 & 2.19506 & 2.26985 & $\mathrm{H}$ & 6.15335 & 0.63784 & -2.29807 \\
\hline C & 2.05746 & 0.95866 & 2.53879 & $\mathrm{H}$ & 5.00014 & -0.52478 & -2.96889 \\
\hline $\mathrm{C}$ & 0.60802 & 0.54087 & 2.40943 & $\mathrm{H}$ & 7.41625 & -1.25908 & -1.20358 \\
\hline $\mathrm{H}$ & 0.51829 & -0.546 & 2.3284 & $\mathrm{H}$ & 6.25797 & -2.41101 & -1.85157 \\
\hline $\mathrm{H}$ & 0.03558 & 0.84247 & 3.29837 & $\mathrm{H}$ & 5.57965 & -0.13401 & 0.07636 \\
\hline $\mathrm{H}$ & 0.12388 & 0.98291 & 1.53495 & $\mathrm{H}$ & 3.78877 & -2.48624 & -0.79581 \\
\hline C & 2.99008 & -0.11165 & 3.04556 & $\mathrm{H}$ & 3.32911 & 0.50408 & -0.29465 \\
\hline $\mathrm{H}$ & 4.01695 & 0.25448 & 3.14849 & $\mathrm{H}$ & 1.62715 & -2.49056 & 0.53162 \\
\hline $\mathrm{H}$ & 2.66152 & -0.49001 & 4.02322 & $\mathrm{Cl}$ & -0.16111 & -4.05192 & 1.69846 \\
\hline $\mathrm{H}$ & 2.99854 & -0.97221 & 2.36393 & $\mathrm{C}$ & -3.25843 & -3.35932 & -1.76006 \\
\hline $\mathrm{H}$ & 3.56461 & 2.39632 & 2.42399 & $\mathrm{H}$ & -2.24714 & -3.61405 & -2.08832 \\
\hline $\mathrm{H}$ & 0.661 & 3.07932 & 1.57937 & $\mathrm{H}$ & -3.78289 & -4.27323 & -1.46995 \\
\hline $\mathrm{H}$ & 1.63882 & 4.12734 & 2.59093 & $\mathrm{H}$ & -3.79488 & -2.87398 & -2.5807 \\
\hline $\mathrm{H}$ & 3.27615 & 4.49132 & 0.82267 & $\mathrm{C}$ & -3.32478 & 1.32169 & -1.04815 \\
\hline $\mathrm{H}$ & 1.66848 & 4.87624 & 0.23178 & $\mathrm{O}$ & -2.6425 & 1.76145 & -1.96863 \\
\hline $\mathrm{H}$ & 0.49511 & 2.55432 & -0.66024 & $\mathrm{C}$ & -3.97534 & 2.28217 & -0.05499 \\
\hline $\mathrm{N}$ & 0.91417 & 0.05913 & -1.52692 & C & -5.12834 & 3.02952 & -0.74328 \\
\hline $\mathrm{C}$ & -0.43115 & -0.08872 & -1.61191 & $\mathrm{H}$ & -4.75612 & 3.59107 & -1.60666 \\
\hline $\mathrm{C}$ & -1.11509 & -1.08002 & -0.95685 & $\mathrm{H}$ & -5.88118 & 2.31305 & -1.08184 \\
\hline $\mathrm{C}$ & -2.55532 & -1.10892 & -0.8815 & $\mathrm{H}$ & -5.61362 & 3.72402 & -0.05139 \\
\hline $\mathrm{C}$ & -3.49805 & -0.12878 & -0.84115 & C & -2.85746 & 3.20896 & 0.46125 \\
\hline $\mathrm{C}$ & -4.76406 & -0.73874 & -0.36312 & $\mathrm{C}$ & -3.31089 & 4.16682 & 1.5556 \\
\hline $\mathrm{O}$ & -5.82724 & -0.20776 & -0.1384 & $\mathrm{H}$ & -3.74042 & 3.61539 & 2.40267 \\
\hline $\mathrm{O}$ & -4.5562 & -2.08048 & -0.18603 & $\mathrm{H}$ & -2.45517 & 4.74444 & 1.91879 \\
\hline C & -3.21098 & -2.41665 & -0.54453 & $\mathrm{H}$ & -4.06994 & 4.86684 & 1.19391 \\
\hline $\mathrm{C}$ & -2.49346 & -3.17762 & 0.60469 & $\mathrm{O}$ & -1.7409 & 2.41896 & 0.91296 \\
\hline $\mathrm{O}$ & -3.13162 & -3.91952 & 1.33335 & $\mathrm{H}$ & -2.00579 & 1.9658 & 1.73 \\
\hline $\mathrm{C}$ & -1.05544 & -3.00896 & 0.6021 & $\mathrm{H}$ & -2.45833 & 3.76616 & -0.39043 \\
\hline $\mathrm{C}$ & -0.38899 & -2.05194 & -0.14748 & $\mathrm{H}$ & -4.38348 & 1.71018 & 0.78594 \\
\hline C & 1.02987 & -1.85068 & -0.10248 & $\mathrm{H}$ & -0.96428 & 0.64015 & -2.20759 \\
\hline
\end{tabular}




\begin{tabular}{|l|r|r|r|r|r|r|r|}
\hline $\mathrm{C}$ & 1.65485 & -0.8086 & -0.7306 & $\mathrm{H}$ & 2.51274 & 1.04171 & -2.46199 \\
\hline $\mathrm{C}$ & 3.08065 & -0.52491 & -0.53335 & $\mathrm{H}$ & 0.9019 & 1.52976 & -2.98757 \\
\hline
\end{tabular}

Table S8. Optimized Z-matrixes of Conf1-6 in the gas phase $(\AA)$ at the B3LYP/6-31G(d) level of theory.

\begin{tabular}{|c|c|c|c|c|c|c|c|}
\hline $\mathrm{C}$ & 1.70131 & 1.5423 & -2.36291 & C & 4.54043 & -0.9424 & -0.7211 \\
\hline C & 1.28045 & 2.694 & -1.49691 & C & 5.9606 & -0.58645 & -0.3744 \\
\hline $\mathrm{C}$ & 2.05195 & 3.59217 & -0.87221 & $\mathrm{C}$ & 6.24311 & 0.91871 & -0.39219 \\
\hline C & 3.54959 & 3.69115 & -0.98658 & $\mathrm{H}$ & 7.30285 & 1.10792 & -0.19365 \\
\hline $\mathrm{H}$ & 4.02393 & 3.56534 & -0.00403 & $\mathrm{H}$ & 5.99501 & 1.36429 & -1.36151 \\
\hline $\mathrm{H}$ & 3.83844 & 4.68771 & -1.34449 & $\mathrm{H}$ & 5.66461 & 1.44036 & 0.37924 \\
\hline $\mathrm{H}$ & 3.98823 & 2.95804 & -1.66764 & C & 6.3467 & -1.19001 & 1.00113 \\
\hline C & 1.4047 & 4.55518 & 0.09748 & C & 6.21491 & -2.7117 & 1.09926 \\
\hline $\mathrm{C}$ & 1.35973 & 3.98057 & 1.53898 & $\mathrm{H}$ & 6.60665 & -3.07225 & 2.05602 \\
\hline $\mathrm{C}$ & 0.44104 & 2.798 & 1.67318 & $\mathrm{H}$ & 6.77672 & -3.21148 & 0.30053 \\
\hline $\mathrm{C}$ & 0.73885 & 1.55009 & 2.06427 & $\mathrm{H}$ & 5.17195 & -3.03855 & 1.0311 \\
\hline $\mathrm{C}$ & 2.12145 & 1.07758 & 2.43956 & $\mathrm{H}$ & 7.38527 & -0.90164 & 1.20939 \\
\hline $\mathrm{H}$ & 2.15861 & 0.79806 & 3.50143 & $\mathrm{H}$ & 5.72809 & -0.71256 & 1.77269 \\
\hline $\mathrm{H}$ & 2.38159 & 0.17545 & 1.87267 & $\mathrm{H}$ & 6.6063 & -1.06782 & -1.12572 \\
\hline $\mathrm{H}$ & 2.90046 & 1.82328 & 2.26219 & $\mathrm{H}$ & 4.34828 & -1.99695 & -0.91145 \\
\hline $\mathrm{C}$ & -0.33508 & 0.50115 & 2.21054 & $\mathrm{H}$ & 3.66174 & 0.9685 & -0.61405 \\
\hline $\mathrm{H}$ & -1.31631 & 0.84108 & 1.87014 & $\mathrm{H}$ & 2.28642 & -2.22228 & 0.26394 \\
\hline $\mathrm{H}$ & -0.0762 & -0.40965 & 1.65968 & $\mathrm{Cl}$ & 0.762 & -4.14544 & 1.22332 \\
\hline $\mathrm{H}$ & -0.43737 & 0.20064 & 3.26276 & $\mathrm{C}$ & -2.48221 & -3.45985 & -2.1066 \\
\hline $\mathrm{H}$ & -0.60111 & 3.00688 & 1.42294 & $\mathrm{H}$ & -2.87664 & -4.46135 & -1.91726 \\
\hline $\mathrm{H}$ & 1.01037 & 4.78403 & 2.20355 & $\mathrm{H}$ & -3.10075 & -2.95848 & -2.8569 \\
\hline $\mathrm{H}$ & 2.37592 & 3.73744 & 1.86626 & $\mathrm{H}$ & -1.45682 & -3.54053 & -2.47683 \\
\hline $\mathrm{H}$ & 1.94917 & 5.50785 & 0.1101 & $\mathrm{C}$ & -3.15348 & 1.06525 & -0.89533 \\
\hline $\mathrm{H}$ & 0.37962 & 4.77464 & -0.22287 & $\mathrm{O}$ & -2.2422 & 1.6792 & -1.45217 \\
\hline $\mathrm{H}$ & 0.20717 & 2.73555 & -1.32078 & $\mathrm{C}$ & -4.32823 & 1.84197 & -0.31123 \\
\hline $\mathrm{N}$ & 1.2685 & 0.2678 & -1.73299 & $\mathrm{C}$ & -4.4764 & 3.19202 & -1.02132 \\
\hline $\mathrm{C}$ & -0.06028 & 0.00113 & -1.75478 & $\mathrm{H}$ & -3.63227 & 3.8512 & -0.79708 \\
\hline $\mathrm{C}$ & -0.6268 & -1.06825 & -1.11064 & $\mathrm{H}$ & -4.50732 & 3.05911 & -2.10687 \\
\hline $\mathrm{C}$ & -2.05288 & -1.25285 & -0.97862 & $\mathrm{H}$ & -5.40069 & 3.6883 & -0.71443 \\
\hline $\mathrm{C}$ & -3.12096 & -0.41159 & -0.80795 & $\mathrm{C}$ & -4.12318 & 2.0119 & 1.22123 \\
\hline $\mathrm{C}$ & -4.25857 & -1.25437 & -0.34668 & $\mathrm{C}$ & -5.30423 & 2.70601 & 1.8982 \\
\hline $\mathrm{O}$ & -5.36347 & -0.9282 & 0.04182 & $\mathrm{H}$ & -5.1331 & 2.72932 & 2.97856 \\
\hline $\mathrm{O}$ & -3.88085 & -2.56019 & -0.36747 & $\mathrm{H}$ & -5.438 & 3.73352 & 1.54638 \\
\hline $\mathrm{C}$ & -2.51936 & -2.66596 & -0.78859 & $\mathrm{H}$ & -6.23333 & 2.15403 & 1.70828 \\
\hline $\mathrm{C}$ & -1.6848 & -3.46477 & 0.25376 & $\mathrm{O}$ & -3.84585 & 0.76801 & 1.85528 \\
\hline $\mathrm{O}$ & -2.21084 & -4.36544 & 0.88509 & $\mathrm{H}$ & -4.59321 & 0.17426 & 1.66108 \\
\hline $\mathrm{C}$ & -0.28249 & -3.11579 & 0.25809 & $\mathrm{H}$ & -3.21494 & 2.60968 & 1.37218 \\
\hline $\mathrm{C}$ & 0.23428 & -2.0151 & -0.4053 & $\mathrm{H}$ & -5.23238 & 1.24112 & -0.44877 \\
\hline $\mathrm{C}$ & 1.61672 & -1.64027 & -0.3532 & $\mathrm{H}$ & -0.68156 & 0.70524 & -2.28383 \\
\hline
\end{tabular}




\begin{tabular}{|l|l|l|l|l|r|r|r|}
\hline $\mathrm{C}$ & 2.11599 & -0.52215 & -0.96411 & $\mathrm{H}$ & 2.77392 & 1.48892 & -2.539 \\
\hline $\mathrm{C}$ & 3.50565 & -0.09217 & -0.77633 & $\mathrm{H}$ & 1.21149 & 1.57974 & -3.34206 \\
\hline
\end{tabular}

Table S9. Optimized Z-matrixes of Conf1-7 in the gas phase $(\AA)$ at the B3LYP/6-31G(d) level of theory.

\begin{tabular}{|c|c|c|c|c|c|c|c|}
\hline $\mathrm{C}$ & 2.06158 & 1.22216 & -2.02708 & C & 4.57017 & -1.19781 & 0.01197 \\
\hline $\mathrm{C}$ & 1.78139 & 2.43875 & -1.19636 & $\mathrm{C}$ & 5.97023 & -0.81621 & 0.39957 \\
\hline $\mathrm{C}$ & 2.65476 & 3.24808 & -0.58245 & $\mathrm{C}$ & 6.41248 & -1.60999 & 1.64037 \\
\hline C & 4.15278 & 3.19136 & -0.7261 & $\mathrm{H}$ & 6.41765 & -2.68697 & 1.43305 \\
\hline $\mathrm{H}$ & 4.50133 & 2.40651 & -1.40223 & $\mathrm{H}$ & 7.42554 & -1.32189 & 1.9422 \\
\hline $\mathrm{H}$ & 4.6332 & 3.03722 & 0.24968 & $\mathrm{H}$ & 5.73886 & -1.43349 & 2.48523 \\
\hline $\mathrm{H}$ & 4.53054 & 4.14865 & -1.10791 & C & 6.94833 & -1.0354 & -0.77579 \\
\hline C & 2.12179 & 4.2516 & 0.41229 & C & 6.64413 & -0.16545 & -1.99688 \\
\hline $\mathrm{C}$ & 2.11239 & 3.68133 & 1.86042 & $\mathrm{H}$ & 7.37826 & -0.32917 & -2.79274 \\
\hline $\mathrm{C}$ & 1.37722 & 2.36806 & 2.00171 & $\mathrm{H}$ & 6.66761 & 0.8999 & -1.73497 \\
\hline $\mathrm{C}$ & 0.04741 & 2.19435 & 2.00551 & $\mathrm{H}$ & 5.65125 & -0.38621 & -2.4038 \\
\hline $\mathrm{C}$ & -0.93952 & 3.32354 & 1.85376 & $\mathrm{H}$ & 7.96495 & -0.82743 & -0.4178 \\
\hline $\mathrm{H}$ & -0.48939 & 4.31062 & 1.9915 & $\mathrm{H}$ & 6.93321 & -2.09685 & -1.06001 \\
\hline $\mathrm{H}$ & -1.3883 & 3.29098 & 0.85128 & $\mathrm{H}$ & 5.97718 & 0.2548 & 0.64806 \\
\hline $\mathrm{H}$ & -1.76346 & 3.21798 & 2.57035 & $\mathrm{H}$ & 4.39471 & -2.25789 & -0.17925 \\
\hline $\mathrm{C}$ & -0.57481 & 0.82879 & 2.13857 & $\mathrm{H}$ & 3.73469 & 0.72645 & 0.03203 \\
\hline $\mathrm{H}$ & -1.15319 & 0.7538 & 3.069 & $\mathrm{H}$ & 2.12422 & -2.32242 & 0.9319 \\
\hline $\mathrm{H}$ & -1.28951 & 0.64108 & 1.33 & $\mathrm{Cl}$ & 0.40529 & -4.13084 & 1.80372 \\
\hline $\mathrm{H}$ & 0.1763 & 0.03372 & 2.13391 & $\mathrm{C}$ & -2.30505 & -3.56243 & -1.98809 \\
\hline $\mathrm{H}$ & 2.00016 & 1.47717 & 2.07604 & $\mathrm{H}$ & -2.74885 & -4.54472 & -1.80766 \\
\hline $\mathrm{H}$ & 1.67444 & 4.44713 & 2.51302 & $\mathrm{H}$ & -2.79583 & -3.09124 & -2.8449 \\
\hline $\mathrm{H}$ & 3.14722 & 3.55169 & 2.20045 & $\mathrm{H}$ & -1.23959 & -3.68227 & -2.2007 \\
\hline $\mathrm{H}$ & 2.71872 & 5.17293 & 0.39657 & $\mathrm{C}$ & -3.02133 & 1.03985 & -1.07735 \\
\hline $\mathrm{H}$ & 1.09659 & 4.5232 & 0.14224 & $\mathrm{O}$ & -1.98344 & 1.62454 & -1.39147 \\
\hline $\mathrm{H}$ & 0.72067 & 2.59342 & -1.00744 & $\mathrm{C}$ & -4.29747 & 1.84105 & -0.84647 \\
\hline $\mathrm{N}$ & 1.46699 & 0.03712 & -1.35777 & $\mathrm{C}$ & -4.27084 & 3.13366 & -1.66925 \\
\hline $\mathrm{C}$ & 0.13569 & -0.1652 & -1.50947 & $\mathrm{H}$ & -3.47135 & 3.79929 & -1.33124 \\
\hline $\mathrm{C}$ & -0.54954 & -1.16472 & -0.86776 & $\mathrm{H}$ & -4.09455 & 2.91941 & -2.72804 \\
\hline $\mathrm{C}$ & -1.98579 & -1.30579 & -0.91846 & $\mathrm{H}$ & -5.22494 & 3.66128 & -1.58699 \\
\hline $\mathrm{C}$ & -3.04372 & -0.43523 & -0.94266 & $\mathrm{C}$ & -4.45409 & 2.131 & 0.67457 \\
\hline $\mathrm{C}$ & -4.25906 & -1.23293 & -0.61737 & $\mathrm{C}$ & -5.82882 & 2.70044 & 1.01899 \\
\hline $\mathrm{O}$ & -5.39964 & -0.86834 & -0.40657 & $\mathrm{H}$ & -6.61675 & 1.99886 & 0.7183 \\
\hline $\mathrm{O}$ & -3.91717 & -2.54504 & -0.52197 & $\mathrm{H}$ & -5.8969 & 2.8518 & 2.10065 \\
\hline $\mathrm{C}$ & -2.51255 & -2.69766 & -0.73212 & $\mathrm{H}$ & -6.01709 & 3.65856 & 0.52428 \\
\hline $\mathrm{C}$ & -1.85943 & -3.45295 & 0.46243 & $\mathrm{O}$ & -4.17704 & 0.97913 & 1.46564 \\
\hline $\mathrm{O}$ & -2.49659 & -4.30532 & 1.05711 & $\mathrm{H}$ & -4.84811 & 0.31307 & 1.23169 \\
\hline $\mathrm{C}$ & -0.46265 & -3.13393 & 0.64871 & $\mathrm{H}$ & -3.67765 & 2.85083 & 0.95456 \\
\hline $\mathrm{C}$ & 0.17574 & -2.09058 & -0.00044 & $\mathrm{H}$ & -5.14549 & 1.21928 & -1.14653 \\
\hline $\mathrm{C}$ & 1.55726 & -1.76246 & 0.20149 & $\mathrm{H}$ & -0.3824 & 0.52912 & -2.15071 \\
\hline
\end{tabular}




\begin{tabular}{|l|l|r|r|l|r|r|r|}
\hline $\mathrm{C}$ & 2.17652 & -0.72141 & -0.43121 & $\mathrm{H}$ & 3.12245 & 1.02756 & -2.18258 \\
\hline $\mathrm{C}$ & 3.55757 & -0.333 & -0.12645 & $\mathrm{H}$ & 1.58937 & 1.28375 & -3.01307 \\
\hline
\end{tabular}

Table S10. Optimized Z-matrixes of Conf1-8 in the gas phase $(\AA)$ at the B3LYP/6-31G(d) level of theory.

\begin{tabular}{|c|c|c|c|c|c|c|c|}
\hline $\mathrm{C}$ & -1.65451 & -0.90778 & -2.7099 & $\mathrm{C}$ & -4.16937 & 0.93492 & -0.12682 \\
\hline $\mathrm{C}$ & -2.50097 & -1.91008 & -1.9749 & $\mathrm{C}$ & -5.66629 & 0.85417 & -0.20051 \\
\hline $\mathrm{C}$ & -2.06367 & -2.98706 & -1.30387 & $\mathrm{C}$ & -6.16304 & -0.41462 & 0.51538 \\
\hline $\mathrm{C}$ & -0.63031 & -3.43141 & -1.2233 & $\mathrm{H}$ & -5.86747 & -0.40928 & 1.57136 \\
\hline $\mathrm{H}$ & -0.53004 & -4.43799 & -1.65069 & $\mathrm{H}$ & -7.25602 & -0.47823 & 0.4724 \\
\hline $\mathrm{H}$ & -0.32088 & -3.51238 & -0.17554 & $\mathrm{H}$ & -5.74737 & -1.31769 & 0.05669 \\
\hline $\mathrm{H}$ & 0.0696 & -2.77252 & -1.73986 & $\mathrm{C}$ & -6.3105 & 2.11931 & 0.40614 \\
\hline $\mathrm{C}$ & -3.07223 & -3.80603 & -0.5247 & $\mathrm{C}$ & -5.93852 & 3.40925 & -0.32733 \\
\hline $\mathrm{C}$ & -3.30717 & -3.24531 & 0.90689 & $\mathrm{H}$ & -6.23166 & 3.35942 & -1.38311 \\
\hline $\mathrm{C}$ & -2.17466 & -3.49198 & 1.86605 & $\mathrm{H}$ & -4.85894 & 3.5918 & -0.29376 \\
\hline $\mathrm{C}$ & -1.173 & -2.66183 & 2.19401 & $\mathrm{H}$ & -6.43974 & 4.27452 & 0.11867 \\
\hline $\mathrm{C}$ & -0.99004 & -1.28268 & 1.61394 & $\mathrm{H}$ & -7.40007 & 1.98685 & 0.39733 \\
\hline $\mathrm{H}$ & -0.91459 & -0.52663 & 2.40641 & $\mathrm{H}$ & -6.01846 & 2.1929 & 1.46315 \\
\hline $\mathrm{H}$ & -0.04768 & -1.23326 & 1.05115 & $\mathrm{H}$ & -5.95946 & 0.79613 & -1.25863 \\
\hline $\mathrm{H}$ & -1.79398 & -0.99268 & 0.93589 & $\mathrm{H}$ & -3.7464 & 1.04409 & 0.87302 \\
\hline $\mathrm{C}$ & -0.0939 & -3.09661 & 3.15264 & $\mathrm{H}$ & -3.75066 & 0.69756 & -2.1694 \\
\hline $\mathrm{H}$ & -0.33505 & -4.05389 & 3.62545 & $\mathrm{H}$ & -1.95928 & 2.56155 & 0.32778 \\
\hline $\mathrm{H}$ & 0.85817 & -3.20602 & 2.61956 & $\mathrm{Cl}$ & -0.27635 & 3.9271 & 1.86476 \\
\hline $\mathrm{H}$ & 0.05633 & -2.34998 & 3.94429 & $\mathrm{C}$ & 2.81952 & 3.59614 & -1.58405 \\
\hline $\mathrm{H}$ & -2.17314 & -4.48149 & 2.32501 & $\mathrm{H}$ & 1.78418 & 3.77214 & -1.88606 \\
\hline $\mathrm{H}$ & -4.21287 & -3.72248 & 1.30394 & $\mathrm{H}$ & 3.2652 & 4.53945 & -1.25857 \\
\hline $\mathrm{H}$ & -3.53158 & -2.17692 & 0.82039 & $\mathrm{H}$ & 3.38489 & 3.20166 & -2.4335 \\
\hline $\mathrm{H}$ & -4.03202 & -3.80085 & -1.05513 & $\mathrm{C}$ & 3.28483 & -1.10887 & -0.92326 \\
\hline $\mathrm{H}$ & -2.7463 & -4.85106 & -0.45532 & $\mathrm{O}$ & 2.3491 & -1.62995 & -1.54818 \\
\hline $\mathrm{H}$ & -3.56832 & -1.70753 & -1.96496 & $\mathrm{C}$ & 4.25891 & -2.00616 & -0.18416 \\
\hline $\mathrm{N}$ & -1.07394 & 0.15108 & -1.83382 & $\mathrm{C}$ & 4.712 & -3.17711 & -1.06442 \\
\hline $\mathrm{C}$ & 0.2735 & 0.25093 & -1.73549 & $\mathrm{H}$ & 3.86032 & -3.78249 & -1.38099 \\
\hline $\mathrm{C}$ & 0.90742 & 1.1591 & -0.92053 & $\mathrm{H}$ & 5.23492 & -2.81561 & -1.95571 \\
\hline $\mathrm{C}$ & 2.34127 & 1.24984 & -0.79219 & $\mathrm{H}$ & 5.40006 & -3.81647 & -0.50175 \\
\hline $\mathrm{C}$ & 3.37177 & 0.34841 & -0.77397 & $\mathrm{C}$ & 3.53397 & -2.51175 & 1.10089 \\
\hline $\mathrm{C}$ & 4.59199 & 1.07281 & -0.31356 & $\mathrm{C}$ & 3.16331 & -1.38149 & 2.06429 \\
\hline $\mathrm{O}$ & 5.71465 & 0.6631 & -0.13558 & $\mathrm{H}$ & 4.03823 & -0.76727 & 2.30551 \\
\hline $\mathrm{O}$ & 4.25896 & 2.38041 & -0.09534 & $\mathrm{H}$ & 2.39431 & -0.72791 & 1.63825 \\
\hline $\mathrm{C}$ & 2.88577 & 2.59729 & -0.41362 & $\mathrm{H}$ & 2.76467 & -1.79906 & 2.99276 \\
\hline $\mathrm{C}$ & 2.12611 & 3.23388 & 0.78713 & $\mathrm{O}$ & 2.39859 & -3.31361 & 0.77865 \\
\hline $\mathrm{O}$ & 2.71753 & 3.95986 & 1.56669 & $\mathrm{H}$ & 1.9905 & -2.93058 & -0.0192 \\
\hline $\mathrm{C}$ & 0.7 & 2.98933 & 0.74851 & $\mathrm{H}$ & 4.23714 & -3.18639 & 1.60425 \\
\hline $\mathrm{C}$ & 0.11005 & 2.06738 & -0.09868 & $\mathrm{H}$ & 5.12322 & -1.42052 & 0.12987 \\
\hline $\mathrm{C}$ & -1.30715 & 1.90023 & -0.2252 & $\mathrm{H}$ & 0.85687 & -0.43967 & -2.32394 \\
\hline
\end{tabular}




\begin{tabular}{|l|r|r|r|l|r|r|r|}
\hline $\mathrm{C}$ & -1.88196 & 0.98052 & -1.05718 & $\mathrm{H}$ & -2.23791 & -0.41169 & -3.49206 \\
\hline $\mathrm{C}$ & -3.33839 & 0.85632 & -1.17488 & $\mathrm{H}$ & -0.8022 & -1.38059 & -3.19924 \\
\hline
\end{tabular}

\section{Computational data of Conf2}

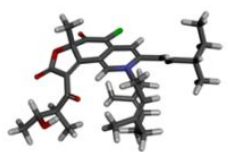

Conf2-1

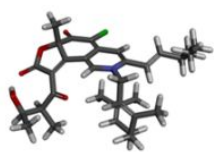

Conf2-2

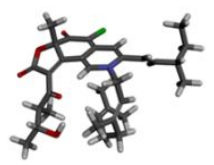

Conf2-3

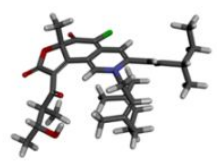

Conf2-4

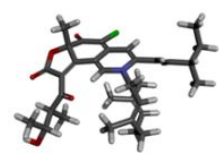

Conf2-5

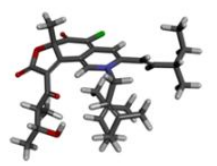

Conf2-6

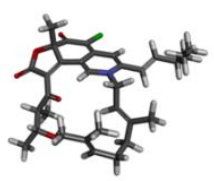

Conf2-7

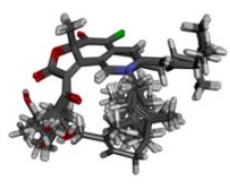

Superimposition of 7 conformers

Figure S2. Optimized geometries of 7 dominant conformers of Conf2 (Conf2-1 to Conf2-7, respectively) at the B3LYP/6-31G(d) level of theory in the gas phase, and the superimposition of 7 dominant conformers.

Table S11. Parameters of the B3LYP/6-31G(d) optimized conformers of Conf2 in the gas phase

\begin{tabular}{|c|c|c|c|}
\hline Conformers & $\mathrm{E}^{a}$ (Hartree) & $\mathrm{C}^{b}($ Hartree $)$ & $\mathrm{G}^{c}(\mathrm{kcal} / \mathrm{mol})$ \\
\hline Conf2-1 & -2174.707957 & 0.616238 & -1364242.553 \\
\hline Conf2-2 & -2174.708659 & 0.61697 & -1364242.535 \\
\hline Conf2-3 & -2174.708807 & 0.617705 & -1364242.166 \\
\hline Conf2-4 & -2174.710703 & 0.619837 & -1364242.018 \\
\hline Conf2-5 & -2174.705327 & 0.614621 & -1364241.918 \\
\hline Conf2-6 & -2174.708923 & 0.618618 & -1364241.667 \\
\hline Conf2-7 & -2174.705039 & 0.616186 & -1364240.755 \\
\hline
\end{tabular}

${ }^{a}$ Electronic energy obtained at B3LYP/ def2-TZVP level of theory; ${ }^{b}$ Thermal correction to Gibbs free energy obtained at B3LYP/6-31G(d) level of theory; ${ }^{C}$ Gibbs free energy $(E+C)$.

Table S12. Conformational analysis of the B3LYP/6-31G(d) optimized conformers of Conf2 (T=298.15 K)

\begin{tabular}{|c|c|c|}
\hline Conformers & $\Delta \mathrm{G}(\mathrm{kcal} / \mathrm{mol})^{a}$ & Population $^{b}$ \\
\hline Conf2-1 & 0 & $28.5 \%$ \\
\hline Conf2-2 & 0.0185489 & $27.6 \%$ \\
\hline Conf2-3 & 0.38697925 & $14.8 \%$ \\
\hline Conf2-4 & 0.53529515 & $11.5 \%$ \\
\hline Conf2-5 & 0.635713975 & $9.7 \%$ \\
\hline Conf2-6 & 0.88694615 & $6.4 \%$ \\
\hline Conf2-7 & 1.798069875 & $1.4 \%$ \\
\hline
\end{tabular}

${ }^{a}$ The relative Gibbs free energy; ${ }^{b}$ The Boltzmann distribution of each conformer.

Table S13. Optimized Z-matrixes of Conf2-1 in the gas phase $(\AA)$ at the B3LYP/6-31G(d) level of theory.

\begin{tabular}{|l|l|l|l|l|r|r|r|}
\hline $\mathrm{C}$ & -1.41442 & -1.8595 & -2.2681 & $\mathrm{C}$ & 3.82289 & -2.27031 & 1.38244 \\
\hline $\mathrm{C}$ & -1.65423 & -2.816 & -1.13739 & $\mathrm{H}$ & 2.98179 & -1.98053 & 2.02005 \\
\hline
\end{tabular}




\begin{tabular}{|c|c|c|c|c|c|c|c|}
\hline $\mathrm{C}$ & -0.76521 & -3.66827 & -0.60812 & $\mathrm{H}$ & 4.62637 & -2.62746 & 2.03219 \\
\hline $\mathrm{C}$ & 0.62976 & -3.90953 & -1.1183 & $\mathrm{H}$ & 3.51237 & -3.09755 & 0.74098 \\
\hline $\mathrm{H}$ & 1.37274 & -3.64066 & -0.35944 & $\mathrm{H}$ & 4.67963 & -0.28557 & 1.19021 \\
\hline $\mathrm{H}$ & 0.76813 & -4.97579 & -1.33877 & $\mathrm{C}$ & 3.89255 & 1.93189 & 0.07073 \\
\hline $\mathrm{H}$ & 0.88074 & -3.34166 & -2.01529 & $\mathrm{O}$ & 5.04834 & 1.80685 & 0.40136 \\
\hline $\mathrm{C}$ & -1.12252 & -4.41674 & 0.65528 & $\mathrm{O}$ & 3.27879 & 3.15208 & 0.02705 \\
\hline $\mathrm{C}$ & -0.67797 & -3.63773 & 1.92196 & $\mathrm{C}$ & 1.95053 & 3.01353 & -0.47457 \\
\hline $\mathrm{C}$ & -1.5362 & -2.43857 & 2.21308 & $\mathrm{C}$ & 0.91519 & 3.65314 & 0.4938 \\
\hline $\mathrm{C}$ & -1.15399 & -1.15817 & 2.32134 & $\mathrm{O}$ & 1.23295 & 4.60201 & 1.18928 \\
\hline $\mathrm{C}$ & 0.26521 & -0.68032 & 2.11787 & $\mathrm{C}$ & -0.4142 & 3.10131 & 0.34379 \\
\hline $\mathrm{H}$ & 0.81662 & -1.29736 & 1.40469 & $\mathrm{C}$ & -0.68584 & 1.95034 & -0.37698 \\
\hline $\mathrm{H}$ & 0.27658 & 0.34981 & 1.74958 & $\mathrm{C}$ & -2.00881 & 1.442 & -0.5837 \\
\hline $\mathrm{H}$ & 0.82126 & -0.68091 & 3.06574 & $\mathrm{C}$ & -2.26509 & 0.25852 & -1.21788 \\
\hline $\mathrm{C}$ & -2.13127 & -0.08768 & 2.73649 & $\mathrm{C}$ & -3.62748 & -0.27404 & -1.33541 \\
\hline $\mathrm{H}$ & -2.16601 & 0.72668 & 2.00292 & $\mathrm{C}$ & -4.51068 & -0.22078 & -0.32937 \\
\hline $\mathrm{H}$ & -3.14234 & -0.48773 & 2.86722 & $\mathrm{C}$ & -5.90933 & -0.76189 & -0.36901 \\
\hline $\mathrm{H}$ & -1.82447 & 0.37154 & 3.68644 & $\mathrm{C}$ & -6.04268 & -1.91335 & 0.64448 \\
\hline $\mathrm{H}$ & -2.58872 & -2.66866 & 2.39165 & $\mathrm{H}$ & -7.05695 & -2.32734 & 0.62849 \\
\hline $\mathrm{H}$ & -0.73808 & -4.3273 & 2.77642 & $\mathrm{H}$ & -5.33731 & -2.72119 & 0.42189 \\
\hline $\mathrm{H}$ & 0.37706 & -3.35963 & 1.83066 & $\mathrm{H}$ & -5.83895 & -1.561 & 1.66302 \\
\hline $\mathrm{H}$ & -0.64223 & -5.40291 & 0.65645 & $\mathrm{C}$ & -6.93916 & 0.35066 & -0.08003 \\
\hline $\mathrm{H}$ & -2.20506 & -4.58449 & 0.70645 & $\mathrm{C}$ & -6.93104 & 1.47629 & -1.11601 \\
\hline $\mathrm{H}$ & -2.62759 & -2.72627 & -0.66206 & $\mathrm{H}$ & -5.95797 & 1.97801 & -1.15324 \\
\hline $\mathrm{N}$ & -1.21075 & -0.47585 & -1.75581 & $\mathrm{H}$ & -7.14011 & 1.08743 & -2.12008 \\
\hline $\mathrm{C}$ & 0.06392 & -0.04822 & -1.57668 & $\mathrm{H}$ & -7.69007 & 2.23068 & -0.88455 \\
\hline $\mathrm{C}$ & 0.38326 & 1.11842 & -0.9261 & $\mathrm{H}$ & -6.74516 & 0.76192 & 0.92059 \\
\hline $\mathrm{C}$ & 1.73869 & 1.53752 & -0.6585 & $\mathrm{H}$ & -7.93551 & -0.10773 & -0.03565 \\
\hline $\mathrm{C}$ & 2.90983 & 0.89617 & -0.36015 & $\mathrm{H}$ & -6.10434 & -1.15686 & -1.37627 \\
\hline $\mathrm{C}$ & 3.19228 & -0.54535 & -0.3308 & $\mathrm{H}$ & -4.19782 & 0.21242 & 0.62082 \\
\hline $\mathrm{O}$ & 2.55547 & -1.3324 & -1.04637 & $\mathrm{H}$ & -3.91455 & -0.74938 & -2.27064 \\
\hline $\mathrm{C}$ & 4.3091 & -1.0808 & 0.5439 & $\mathrm{H}$ & -2.84987 & 2.02061 & -0.2285 \\
\hline $\mathrm{C}$ & 5.47028 & -1.46997 & -0.42868 & $\mathrm{Cl}$ & -1.70463 & 3.96024 & 1.16748 \\
\hline $\mathrm{O}$ & 5.12889 & -2.60901 & -1.20627 & $\mathrm{C}$ & 1.86606 & 3.77521 & -1.8111 \\
\hline $\mathrm{H}$ & 4.21706 & -2.47346 & -1.5217 & $\mathrm{H}$ & 2.61432 & 3.38104 & -2.50498 \\
\hline $\mathrm{C}$ & 6.76513 & -1.77859 & 0.3083 & $\mathrm{H}$ & 0.87179 & 3.67517 & -2.25344 \\
\hline $\mathrm{H}$ & 7.54167 & -2.04127 & -0.41656 & $\mathrm{H}$ & 2.07029 & 4.83156 & -1.6193 \\
\hline $\mathrm{H}$ & 6.63967 & -2.62582 & 0.98934 & $\mathrm{H}$ & 0.8499 & -0.69083 & -1.9452 \\
\hline $\mathrm{H}$ & 7.09586 & -0.90384 & 0.87653 & $\mathrm{H}$ & -2.24988 & -1.83978 & -2.97171 \\
\hline $\mathrm{H}$ & 5.65061 & -0.60121 & -1.08223 & $\mathrm{H}$ & -0.52053 & -2.1073 & -2.8395 \\
\hline
\end{tabular}

Table S14. Optimized Z-matrixes of Conf2-2 in the gas phase $(\AA)$ at the B3LYP/6-31G(d) level of theory.

\begin{tabular}{|l|r|r|r|l|r|r|r|}
\hline $\mathrm{C}$ & -1.7479 & -1.435 & -1.97675 & $\mathrm{C}$ & 3.44256 & -2.88505 & 1.52333 \\
\hline $\mathrm{C}$ & -1.53214 & -2.59244 & -1.04719 & $\mathrm{H}$ & 4.19479 & -3.50579 & 2.02247 \\
\hline
\end{tabular}




\begin{tabular}{|c|c|c|c|c|c|c|c|}
\hline $\mathrm{C}$ & -2.4546 & -3.35754 & -0.44908 & $\mathrm{H}$ & 2.75772 & -3.53685 & 0.97614 \\
\hline $\mathrm{C}$ & -3.94005 & -3.28908 & -0.68803 & $\mathrm{H}$ & 2.87137 & -2.3651 & 2.29954 \\
\hline $\mathrm{H}$ & -4.23601 & -2.53749 & -1.42395 & $\mathrm{H}$ & 4.6682 & -1.14746 & 1.17213 \\
\hline $\mathrm{H}$ & -4.30951 & -4.26062 & -1.04074 & $\mathrm{C}$ & 4.4146 & 1.11924 & -0.17166 \\
\hline $\mathrm{H}$ & -4.47507 & -3.07685 & 0.24787 & $\mathrm{O}$ & 5.54995 & 0.76773 & 0.08611 \\
\hline $\mathrm{C}$ & -2.0088 & -4.3282 & 0.62181 & $\mathrm{O}$ & 4.08957 & 2.43791 & -0.23617 \\
\hline $\mathrm{C}$ & -1.94977 & -3.65049 & 2.01725 & $\mathrm{C}$ & 2.71887 & 2.58458 & -0.61624 \\
\hline $\mathrm{C}$ & -0.88477 & -2.59252 & 2.0987 & $\mathrm{C}$ & 1.94773 & 3.4763 & 0.40128 \\
\hline $\mathrm{C}$ & -1.00024 & -1.31411 & 2.48687 & $\mathrm{O}$ & 2.52964 & 4.38103 & 0.97452 \\
\hline $\mathrm{C}$ & -2.27996 & -0.66347 & 2.94675 & $\mathrm{C}$ & 0.5291 & 3.19587 & 0.45356 \\
\hline $\mathrm{H}$ & -2.48513 & 0.23868 & 2.35693 & $\mathrm{C}$ & -0.0557 & 2.08823 & -0.1386 \\
\hline $\mathrm{H}$ & -3.15075 & -1.31982 & 2.87678 & $\mathrm{C}$ & -1.45487 & 1.78551 & -0.0547 \\
\hline $\mathrm{H}$ & -2.19075 & -0.33742 & 3.99211 & $\mathrm{C}$ & -2.00944 & 0.66672 & -0.61293 \\
\hline $\mathrm{C}$ & 0.20972 & -0.413 & 2.53709 & $\mathrm{C}$ & -3.41228 & 0.3002 & -0.39389 \\
\hline $\mathrm{H}$ & 0.48046 & -0.17757 & 3.57589 & $\mathrm{C}$ & -4.43552 & 1.16201 & -0.4418 \\
\hline $\mathrm{H}$ & 1.07651 & -0.87246 & 2.05389 & $\mathrm{C}$ & -5.86086 & 0.81079 & -0.1246 \\
\hline $\mathrm{H}$ & 0.01298 & 0.54568 & 2.04554 & $\mathrm{C}$ & -6.39229 & 1.7243 & 0.99297 \\
\hline $\mathrm{H}$ & 0.10805 & -2.93257 & 1.79853 & $\mathrm{H}$ & -5.78192 & 1.63829 & 1.89788 \\
\hline $\mathrm{H}$ & -2.93611 & -3.24684 & 2.26922 & $\mathrm{H}$ & -6.38143 & 2.7746 & 0.67741 \\
\hline $\mathrm{H}$ & -1.7416 & -4.43621 & 2.75828 & $\mathrm{H}$ & -7.42491 & 1.46145 & 1.24781 \\
\hline $\mathrm{H}$ & -1.01321 & -4.71938 & 0.38206 & $\mathrm{C}$ & -6.74688 & 0.90056 & -1.38613 \\
\hline $\mathrm{H}$ & -2.68908 & -5.18737 & 0.66965 & $\mathrm{C}$ & -6.35488 & -0.09547 & -2.47915 \\
\hline $\mathrm{H}$ & -0.48673 & -2.75026 & -0.78705 & $\mathrm{H}$ & -5.33139 & 0.08091 & -2.82809 \\
\hline $\mathrm{N}$ & -1.21297 & -0.19522 & -1.35851 & $\mathrm{H}$ & -6.40675 & -1.12628 & -2.10655 \\
\hline $\mathrm{C}$ & 0.12918 & -0.00787 & -1.3904 & $\mathrm{H}$ & -7.02292 & -0.01935 & -3.34337 \\
\hline $\mathrm{C}$ & 0.74796 & 1.06072 & -0.79475 & $\mathrm{H}$ & -6.7044 & 1.92524 & -1.78095 \\
\hline $\mathrm{C}$ & 2.18055 & 1.18842 & -0.68937 & $\mathrm{H}$ & -7.78873 & 0.72952 & -1.08592 \\
\hline $\mathrm{C}$ & 3.1999 & 0.30679 & -0.45558 & $\mathrm{H}$ & -5.88658 & -0.22883 & 0.23253 \\
\hline $\mathrm{C}$ & 3.09303 & -1.15941 & -0.27816 & $\mathrm{H}$ & -4.24811 & 2.19545 & -0.73835 \\
\hline $\mathrm{O}$ & 2.16117 & -1.78507 & -0.78392 & $\mathrm{H}$ & -3.59773 & $\begin{array}{l}-0.73559 \\
\end{array}$ & -0.12567 \\
\hline $\mathrm{C}$ & 4.12146 & -1.88575 & 0.58007 & $\mathrm{H}$ & -2.09228 & 2.42683 & 0.53791 \\
\hline $\mathrm{C}$ & 5.17097 & -2.56139 & -0.35747 & $\mathrm{Cl}$ & -0.44467 & 4.32874 & 1.37546 \\
\hline $\mathrm{O}$ & 5.64898 & -1.63523 & -1.32063 & $\mathrm{C}$ & 2.67544 & 3.29678 & -1.97953 \\
\hline $\mathrm{H}$ & 5.91646 & -0.82611 & -0.84257 & $\mathrm{H}$ & 1.64327 & 3.40139 & -2.32384 \\
\hline $\mathrm{C}$ & 4.64417 & -3.76244 & -1.131 & $\mathrm{H}$ & 3.11997 & 4.28869 & -1.86663 \\
\hline $\mathrm{H}$ & 5.43277 & -4.12185 & -1.79896 & $\mathrm{H}$ & 3.24666 & 2.72148 & -2.714 \\
\hline $\mathrm{H}$ & 3.77617 & -3.4859 & -1.7357 & $\mathrm{H}$ & 0.70927 & -0.76562 & -1.89246 \\
\hline $\mathrm{H}$ & 4.3596 & -4.57762 & -0.45918 & $\mathrm{H}$ & -2.79358 & -1.26208 & -2.22734 \\
\hline $\mathrm{H}$ & 5.99215 & -2.89797 & 0.29898 & $\mathrm{H}$ & -1.20182 & -1.56671 & -2.91677 \\
\hline
\end{tabular}

Table S15. Optimized Z-matrixes of Conf2-3 in the gas phase $(\AA)$ at the B3LYP/6-31G(d) level of theory.

\begin{tabular}{|l|r|r|r|l|r|r|r|}
\hline $\mathrm{C}$ & -1.44452 & -1.7116 & -2.35089 & $\mathrm{C}$ & 3.87594 & -0.63153 & 1.67332 \\
\hline $\mathrm{C}$ & -1.3516 & -2.70603 & -1.23272 & $\mathrm{H}$ & 4.73362 & -0.84486 & 2.31617 \\
\hline
\end{tabular}




\begin{tabular}{|c|c|c|c|c|c|c|c|}
\hline $\mathrm{C}$ & -0.40945 & -3.64038 & -1.04266 & $\mathrm{H}$ & 3.08402 & -1.34996 & 1.89834 \\
\hline $\mathrm{C}$ & 0.69732 & -3.97497 & -2.0083 & $\mathrm{H}$ & 3.52298 & 0.37244 & 1.92375 \\
\hline $\mathrm{H}$ & 1.64942 & -4.11256 & -1.48552 & $\mathrm{H}$ & 5.1401 & -0.04096 & 0.04502 \\
\hline $\mathrm{H}$ & 0.4822 & -4.92917 & -2.50823 & $\mathrm{C}$ & 3.75325 & 2.2902 & -0.16215 \\
\hline $\mathrm{H}$ & 0.84965 & -3.21992 & -2.78362 & $\mathrm{O}$ & 4.92106 & 2.21207 & 0.13716 \\
\hline $\mathrm{C}$ & -0.39819 & -4.4027 & 0.26198 & $\mathrm{O}$ & 3.07299 & 3.47473 & -0.12325 \\
\hline $\mathrm{C}$ & 0.64043 & -3.82323 & 1.26508 & $\mathrm{C}$ & 1.72697 & 3.28196 & -0.55642 \\
\hline $\mathrm{C}$ & 0.51701 & -2.33228 & 1.4409 & $\mathrm{C}$ & 0.71933 & 3.81455 & 0.50182 \\
\hline $\mathrm{C}$ & -0.22756 & -1.66684 & 2.33542 & $\mathrm{O}$ & 1.02486 & 4.75425 & 1.21499 \\
\hline $\mathrm{C}$ & -1.1154 & -2.331 & 3.35592 & $\mathrm{C}$ & -0.58082 & 3.18509 & 0.41667 \\
\hline $\mathrm{H}$ & -0.85891 & -2.00462 & 4.37273 & $\mathrm{C}$ & -0.83015 & 2.04622 & -0.32864 \\
\hline $\mathrm{H}$ & -2.16447 & -2.04401 & 3.19451 & $\mathrm{C}$ & -2.12836 & 1.45208 & -0.45239 \\
\hline $\mathrm{H}$ & -1.0601 & -3.42191 & 3.32524 & $\mathrm{C}$ & -2.35842 & 0.29153 & -1.13703 \\
\hline $\mathrm{C}$ & -0.23406 & -0.15952 & 2.37043 & $\mathrm{C}$ & -3.69057 & -0.32527 & -1.1777 \\
\hline $\mathrm{H}$ & 0.45364 & 0.26695 & 1.63567 & $\mathrm{C}$ & -4.48087 & -0.40588 & -0.09875 \\
\hline $\mathrm{H}$ & -1.23545 & 0.24405 & 2.16676 & $\mathrm{C}$ & -5.85935 & -0.99768 & -0.07088 \\
\hline $\mathrm{H}$ & 0.055 & 0.21427 & 3.36189 & $\mathrm{C}$ & -5.89629 & -2.19398 & 0.89609 \\
\hline $\mathrm{H}$ & 1.08044 & -1.73917 & 0.72483 & $\mathrm{H}$ & -5.64539 & -1.87876 & 1.91623 \\
\hline $\mathrm{H}$ & 0.51417 & -4.34985 & 2.21738 & $\mathrm{H}$ & -6.89631 & -2.64077 & 0.91955 \\
\hline $\mathrm{H}$ & 1.6564 & -4.04481 & 0.92177 & $\mathrm{H}$ & -5.18085 & -2.96751 & 0.59748 \\
\hline $\mathrm{H}$ & -0.17189 & -5.46317 & 0.08794 & $\mathrm{C}$ & -6.90357 & 0.06848 & 0.32793 \\
\hline $\mathrm{H}$ & -1.39088 & -4.35008 & 0.72296 & $\mathrm{C}$ & -7.00419 & 1.23084 & -0.66134 \\
\hline $\mathrm{H}$ & -2.1039 & -2.57158 & -0.45824 & $\mathrm{H}$ & -6.05696 & 1.77538 & -0.7384 \\
\hline $\mathrm{N}$ & -1.30829 & -0.33283 & -1.80733 & $\mathrm{H}$ & -7.26056 & 0.87114 & -1.66536 \\
\hline $\mathrm{C}$ & -0.05201 & 0.16822 & -1.6929 & $\mathrm{H}$ & -7.77716 & 1.9428 & -0.35395 \\
\hline $\mathrm{C}$ & 0.24446 & 1.31399 & -0.99754 & $\mathrm{H}$ & -6.65721 & 0.4494 & 1.329 \\
\hline $\mathrm{C}$ & 1.58949 & 1.80637 & -0.80205 & $\mathrm{H}$ & -7.87875 & -0.4271 & 0.41951 \\
\hline $\mathrm{C}$ & 2.81269 & 1.22425 & -0.61439 & $\mathrm{H}$ & -6.10591 & -1.3545 & -1.08107 \\
\hline $\mathrm{C}$ & 3.16836 & -0.20197 & -0.68804 & $\mathrm{H}$ & -4.105 & -0.03072 & 0.85469 \\
\hline $\mathrm{O}$ & 2.52865 & -0.96132 & -1.42955 & $\mathrm{H}$ & -4.03973 & -0.73311 & -2.12375 \\
\hline $\mathrm{C}$ & 4.29459 & -0.72395 & 0.18957 & $\mathrm{H}$ & -2.97236 & 1.94724 & 0.00665 \\
\hline $\mathrm{C}$ & 4.7123 & -2.14581 & -0.24624 & $\mathrm{Cl}$ & -1.85773 & 3.92507 & 1.3667 \\
\hline $\mathrm{O}$ & 3.63012 & -3.07053 & -0.1275 & $\mathrm{C}$ & 1.51834 & 4.0987 & -1.84527 \\
\hline $\mathrm{H}$ & 2.93651 & -2.68291 & -0.69077 & $\mathrm{H}$ & 0.50619 & 3.95826 & -2.2328 \\
\hline $\mathrm{C}$ & 5.87665 & -2.70048 & 0.56008 & $\mathrm{H}$ & 1.67145 & 5.15543 & -1.61248 \\
\hline $\mathrm{H}$ & 6.73541 & -2.02125 & 0.5268 & $\mathrm{H}$ & 2.24435 & 3.78379 & -2.60062 \\
\hline $\mathrm{H}$ & 6.17954 & -3.66885 & 0.15073 & $\mathrm{H}$ & 0.73897 & -0.40123 & -2.15685 \\
\hline $\mathrm{H}$ & 5.58829 & -2.85224 & 1.60433 & $\mathrm{H}$ & -2.39606 & -1.77188 & -2.88363 \\
\hline $\mathrm{H}$ & 5.01757 & -2.08177 & -1.30345 & $\mathrm{H}$ & -0.65458 & -1.83609 & -3.09192 \\
\hline
\end{tabular}

Table S16. Optimized Z-matrixes of Conf2-4 in the gas phase $(\AA)$ at the B3LYP/6-31G(d) level of theory.

\begin{tabular}{|l|r|r|r|r|r|r|r|}
\hline $\mathrm{C}$ & -1.28566 & -1.77614 & -2.42184 & $\mathrm{C}$ & 3.8087 & -1.29586 & 1.53479 \\
\hline $\mathrm{C}$ & -1.72331 & -2.74992 & -1.36086 & $\mathrm{H}$ & 3.36472 & -0.35313 & 1.86778 \\
\hline
\end{tabular}




\begin{tabular}{|c|c|c|c|c|c|c|c|}
\hline $\mathrm{C}$ & -0.90331 & -3.34494 & -0.48212 & $\mathrm{H}$ & 4.56868 & -1.58013 & 2.26697 \\
\hline $\mathrm{C}$ & 0.59338 & -3.18142 & -0.49457 & $\mathrm{H}$ & 3.03539 & -2.06911 & 1.52317 \\
\hline $\mathrm{H}$ & 1.09858 & -4.07899 & -0.12385 & $\mathrm{H}$ & 5.28678 & -0.46886 & 0.21141 \\
\hline $\mathrm{H}$ & 0.98356 & -2.96419 & -1.49114 & $\mathrm{C}$ & 4.08413 & 1.89819 & $\begin{array}{l}-0.03704 \\
\end{array}$ \\
\hline $\mathrm{H}$ & 0.90621 & -2.3541 & 0.15202 & $\mathrm{O}$ & 5.23335 & 1.74039 & 0.3017 \\
\hline $\mathrm{C}$ & -1.46183 & -4.17813 & 0.64244 & $\mathrm{O}$ & 3.46589 & 3.11303 & 0.0504 \\
\hline $\mathrm{C}$ & -1.17856 & -3.55039 & 2.03094 & $\mathrm{C}$ & 2.13059 & 3.01915 & -0.44344 \\
\hline $\mathrm{C}$ & -1.89053 & -2.24453 & 2.24985 & $\mathrm{C}$ & 1.11531 & 3.57851 & 0.58835 \\
\hline $\mathrm{C}$ & -1.36423 & -1.02987 & 2.46543 & $\mathrm{O}$ & 1.44536 & 4.46979 & 1.35182 \\
\hline $\mathrm{C}$ & 0.10995 & -0.71359 & 2.48251 & $\mathrm{C}$ & -0.2169 & 3.04181 & 0.41693 \\
\hline $\mathrm{H}$ & 0.35537 & 0.0104 & 1.69426 & $\mathrm{C}$ & -0.50095 & 1.9414 & $\begin{array}{l}-0.37409 \\
\end{array}$ \\
\hline $\mathrm{H}$ & 0.39358 & -0.24035 & 3.43173 & $\mathrm{C}$ & -1.82937 & 1.45165 & -0.59243 \\
\hline $\mathrm{H}$ & 0.74518 & -1.59044 & 2.34516 & $\mathrm{C}$ & -2.10018 & 0.29916 & -1.27411 \\
\hline $\mathrm{C}$ & -2.25026 & 0.15546 & 2.75556 & $\mathrm{C}$ & -3.46512 & -0.21662 & -1.41544 \\
\hline $\mathrm{H}$ & -2.04568 & 0.55529 & 3.75816 & $\mathrm{C}$ & -4.3682 & -0.16269 & $\begin{array}{l}-0.42769 \\
\end{array}$ \\
\hline $\mathrm{H}$ & -2.06345 & 0.97928 & 2.05749 & $\mathrm{C}$ & -5.76792 & -0.6969 & -0.50004 \\
\hline $\mathrm{H}$ & -3.31167 & -0.10974 & 2.71134 & $\mathrm{C}$ & -5.92003 & -1.86749 & 0.48898 \\
\hline $\mathrm{H}$ & -2.97961 & -2.3237 & 2.25049 & $\mathrm{H}$ & -5.73715 & -1.53377 & 1.5177 \\
\hline $\mathrm{H}$ & -1.51276 & -4.26914 & 2.79236 & $\mathrm{H}$ & -6.93304 & -2.28266 & 0.44526 \\
\hline $\mathrm{H}$ & -0.09822 & -3.44497 & 2.16753 & $\mathrm{H}$ & -5.20798 & -2.66944 & 0.26639 \\
\hline $\mathrm{H}$ & -1.01054 & -5.17985 & 0.61745 & $\mathrm{C}$ & -6.79932 & 0.41248 & -0.20563 \\
\hline $\mathrm{H}$ & -2.54385 & -4.30549 & 0.51629 & $\mathrm{C}$ & -6.76718 & 1.56108 & -1.2156 \\
\hline $\mathrm{H}$ & -2.79416 & -2.90073 & -1.26119 & $\mathrm{H}$ & -5.79122 & 2.05844 & -1.22312 \\
\hline $\mathrm{N}$ & -1.05574 & -0.41508 & -1.8585 & $\mathrm{H}$ & -6.95899 & 1.19601 & -2.23197 \\
\hline $\mathrm{C}$ & 0.21919 & 0.02389 & -1.7158 & $\mathrm{H}$ & -7.52705 & 2.31377 & -0.98133 \\
\hline $\mathrm{C}$ & 0.55538 & 1.15473 & -1.00966 & $\mathrm{H}$ & -6.62209 & 0.80071 & 0.80729 \\
\hline $\mathrm{C}$ & 1.9194 & 1.56478 & -0.76359 & $\mathrm{H}$ & -7.79815 & -0.04236 & -0.18968 \\
\hline $\mathrm{C}$ & 3.10903 & 0.91352 & -0.5906 & $\mathrm{H}$ & -5.94822 & -1.07226 & -1.5176 \\
\hline $\mathrm{C}$ & 3.42817 & -0.51201 & -0.79298 & $\mathrm{H}$ & -4.07214 & 0.2597 & 0.53194 \\
\hline $\mathrm{O}$ & 2.85231 & -1.15962 & $\begin{array}{l}-1.67599 \\
\end{array}$ & $\mathrm{H}$ & -3.73758 & -0.68258 & -2.35984 \\
\hline $\mathrm{C}$ & 4.44186 & -1.16155 & 0.13275 & $\mathrm{H}$ & -2.6626 & 2.01997 & -0.20405 \\
\hline $\mathrm{C}$ & 4.93692 & -2.50782 & -0.43801 & $\mathrm{Cl}$ & -1.49326 & 3.85006 & 1.31237 \\
\hline $\mathrm{O}$ & 3.87661 & -3.45447 & -0.55853 & $\mathrm{C}$ & 2.02773 & 3.8913 & -1.70996 \\
\hline $\mathrm{H}$ & 3.2607 & -3.04157 & -1.18827 & $\mathrm{H}$ & 2.76698 & 3.55735 & -2.44394 \\
\hline $\mathrm{C}$ & 6.02181 & -3.14763 & 0.41647 & $\mathrm{H}$ & 1.02771 & 3.82637 & -2.14597 \\
\hline $\mathrm{H}$ & 6.85798 & -2.45706 & 0.57022 & $\mathrm{H}$ & 2.23312 & 4.92816 & -1.43217 \\
\hline $\mathrm{H}$ & 6.39637 & -4.04882 & -0.07783 & $\mathrm{H}$ & 0.99639 & -0.57158 & -2.17316 \\
\hline $\mathrm{H}$ & 5.62421 & -3.44134 & 1.39268 & $\mathrm{H}$ & -2.02473 & -1.69707 & -3.22251 \\
\hline $\mathrm{H}$ & 5.35641 & -2.2997 & -1.43583 & $\mathrm{H}$ & -0.34184 & -2.06838 & -2.88493 \\
\hline
\end{tabular}

Table S17. Optimized Z-matrixes of Conf2-5 in the gas phase $(\AA)$ at the B3LYP/6-31G(d) level of theory.

\begin{tabular}{|l|l|l|l|l|r|r|r|}
\hline $\mathrm{C}$ & -1.30354 & -1.83788 & -2.28402 & $\mathrm{C}$ & 3.75756 & -1.28812 & 1.71905 \\
\hline $\mathrm{C}$ & -1.64607 & -2.77914 & -1.16441 & $\mathrm{H}$ & 3.42969 & -0.33507 & 2.14488 \\
\hline
\end{tabular}




\begin{tabular}{|c|c|c|c|c|c|c|c|}
\hline $\mathrm{C}$ & -0.79888 & -3.58479 & -0.5081 & $\mathrm{H}$ & 4.49235 & -1.74165 & 2.38697 \\
\hline $\mathrm{C}$ & 0.66372 & -3.76115 & -0.81946 & $\mathrm{H}$ & 2.89181 & -1.9573 & 1.65909 \\
\hline $\mathrm{H}$ & 1.04428 & -3.10695 & -1.60322 & $\mathrm{H}$ & 5.19522 & -0.35541 & 0.42304 \\
\hline $\mathrm{H}$ & 1.27302 & -3.56737 & 0.07165 & $\mathrm{C}$ & 3.97765 & 1.94664 & 0.05997 \\
\hline $\mathrm{H}$ & 0.86219 & -4.80149 & -1.10965 & $\mathrm{O}$ & 5.1259 & 1.81489 & 0.41537 \\
\hline $\mathrm{C}$ & -1.29688 & -4.36718 & 0.68483 & $\mathrm{O}$ & 3.35167 & 3.16042 & 0.09544 \\
\hline $\mathrm{C}$ & -0.96932 & -3.65417 & 2.02406 & $\mathrm{C}$ & 2.02521 & 3.04153 & -0.41526 \\
\hline $\mathrm{C}$ & -1.79966 & -2.42651 & 2.27774 & $\mathrm{C}$ & 0.98849 & 3.62576 & 0.58299 \\
\hline $\mathrm{C}$ & -1.36918 & -1.15764 & 2.31635 & $\mathrm{O}$ & 1.30147 & 4.5393 & 1.32704 \\
\hline $\mathrm{C}$ & 0.06575 & -0.75968 & 2.04269 & $\mathrm{C}$ & -0.33829 & 3.07772 & 0.40323 \\
\hline $\mathrm{H}$ & 0.49244 & -1.31558 & 1.20312 & $\mathrm{C}$ & -0.60251 & 1.95744 & -0.3667 \\
\hline $\mathrm{H}$ & 0.13674 & 0.30565 & 1.80496 & $\mathrm{C}$ & -1.92393 & 1.45466 & -0.59843 \\
\hline $\mathrm{H}$ & 0.70615 & -0.93466 & 2.91717 & $\mathrm{C}$ & -2.17438 & 0.27944 & -1.24977 \\
\hline $\mathrm{C}$ & -2.28777 & -0.03065 & 2.71179 & $\mathrm{C}$ & -3.53399 & -0.24976 & -1.40018 \\
\hline $\mathrm{H}$ & -2.29964 & 0.7609 & 1.95359 & $\mathrm{C}$ & -4.44786 & -0.18984 & -0.4225 \\
\hline $\mathrm{H}$ & -3.31286 & -0.37812 & 2.87831 & $\mathrm{C}$ & -5.84245 & -0.73711 & -0.50053 \\
\hline $\mathrm{H}$ & -1.93841 & 0.44508 & 3.63869 & $\mathrm{C}$ & -5.99684 & -1.88928 & 0.50955 \\
\hline $\mathrm{H}$ & -2.8543 & -2.61629 & 2.48558 & $\mathrm{H}$ & -7.00807 & -2.30884 & 0.46642 \\
\hline $\mathrm{H}$ & -1.14819 & -4.37258 & 2.83607 & $\mathrm{H}$ & -5.28085 & -2.6931 & 0.30738 \\
\hline $\mathrm{H}$ & 0.09902 & -3.41674 & 2.05979 & $\mathrm{H}$ & -5.82208 & -1.53536 & 1.53288 \\
\hline $\mathrm{H}$ & -0.83656 & -5.3637 & 0.69408 & $\mathrm{C}$ & -6.8855 & 0.36961 & -0.23901 \\
\hline $\mathrm{H}$ & -2.38157 & -4.51263 & 0.61777 & $\mathrm{C}$ & -6.851 & 1.49918 & -1.27018 \\
\hline $\mathrm{H}$ & -2.67755 & -2.73778 & -0.8258 & $\mathrm{H}$ & -5.87916 & 2.0046 & -1.27605 \\
\hline $\mathrm{N}$ & -1.11389 & -0.4469 & -1.78634 & $\mathrm{H}$ & -7.028 & 1.11338 & -2.28158 \\
\hline $\mathrm{C}$ & 0.15558 & 0.00148 & -1.6252 & $\mathrm{H}$ & -7.61967 & 2.24979 & -1.05898 \\
\hline $\mathrm{C}$ & 0.47064 & 1.15717 & -0.95331 & $\mathrm{H}$ & -6.72299 & 0.77816 & 0.76832 \\
\hline $\mathrm{C}$ & 1.82722 & 1.57635 & -0.68665 & $\mathrm{H}$ & -7.88087 & -0.09287 & -0.22581 \\
\hline $\mathrm{C}$ & 3.01451 & 0.93342 & -0.46371 & $\mathrm{H}$ & -6.00809 & -1.1333 & -1.51261 \\
\hline $\mathrm{C}$ & 3.32723 & -0.50563 & -0.58464 & $\mathrm{H}$ & -4.16636 & 0.24998 & 0.53393 \\
\hline $\mathrm{O}$ & 2.69842 & -1.22711 & -1.36215 & $\mathrm{H}$ & -3.79101 & -0.73425 & -2.33972 \\
\hline $\mathrm{C}$ & 4.39084 & -1.09231 & 0.3251 & $\mathrm{H}$ & -2.7682 & 2.03019 & -0.24561 \\
\hline $\mathrm{C}$ & 4.97106 & -2.40826 & -0.22826 & $\mathrm{Cl}$ & -1.63493 & 3.89438 & 1.26084 \\
\hline $\mathrm{O}$ & 5.84553 & -3.00668 & 0.73201 & $\mathrm{C}$ & 1.93741 & 3.873 & -1.70976 \\
\hline $\mathrm{H}$ & 6.61306 & -2.41815 & 0.82361 & $\mathrm{H}$ & 0.9451 & 3.78813 & -2.15968 \\
\hline $\mathrm{C}$ & 5.68789 & -2.21847 & -1.5641 & $\mathrm{H}$ & 2.13221 & 4.9194 & -1.4616 \\
\hline $\mathrm{H}$ & 6.13288 & -3.16535 & -1.8845 & $\mathrm{H}$ & 2.6905 & 3.52144 & -2.42115 \\
\hline $\mathrm{H}$ & 6.49146 & -1.47565 & -1.46755 & $\mathrm{H}$ & 0.94746 & -0.61393 & -2.02623 \\
\hline $\mathrm{H}$ & 4.99862 & -1.87485 & -2.33997 & $\mathrm{H}$ & -2.08454 & -1.82376 & -3.04855 \\
\hline $\mathrm{H}$ & 4.15841 & -3.13063 & -0.34953 & $\mathrm{H}$ & -0.37289 & -2.11104 & -2.78023 \\
\hline
\end{tabular}

Table S18. Optimized Z-matrixes of Conf2-6 in the gas phase $(\AA)$ at the B3LYP/6-31G(d) level of theory.

\begin{tabular}{|l|r|r|r|r|r|r|r|}
\hline $\mathrm{C}$ & -1.46071 & -1.47191 & -2.56845 & $\mathrm{C}$ & 3.89199 & -1.0128 & 1.62402 \\
\hline $\mathrm{C}$ & -0.75078 & -2.58957 & -1.85698 & $\mathrm{H}$ & 3.0192 & -1.60557 & 1.90842 \\
\hline
\end{tabular}




\begin{tabular}{|c|c|c|c|c|c|c|c|}
\hline $\mathrm{C}$ & -1.27324 & -3.41301 & -0.93779 & $\mathrm{H}$ & 3.69156 & 0.03865 & 1.84201 \\
\hline $\mathrm{C}$ & -2.71662 & -3.41474 & -0.50981 & $\mathrm{H}$ & 4.73417 & -1.31823 & 2.24986 \\
\hline $\mathrm{H}$ & -3.08677 & -4.44247 & -0.41087 & $\mathrm{H}$ & 5.18611 & -0.70936 & -0.0557 \\
\hline $\mathrm{H}$ & -2.81988 & -2.93865 & 0.47244 & $\mathrm{C}$ & 4.10005 & 1.83836 & -0.20022 \\
\hline $\mathrm{H}$ & -3.37414 & -2.87936 & -1.19684 & $\mathrm{O}$ & 5.25225 & 1.61349 & 0.08439 \\
\hline $\mathrm{C}$ & -0.37107 & -4.36024 & -0.18458 & $\mathrm{O}$ & 3.56091 & 3.09226 & -0.11939 \\
\hline $\mathrm{C}$ & -0.25257 & -3.97479 & 1.31329 & $\mathrm{C}$ & 2.19567 & 3.06816 & -0.53892 \\
\hline $\mathrm{C}$ & 0.16183 & -2.5416 & 1.49357 & $\mathrm{C}$ & 1.26072 & 3.6465 & 0.56565 \\
\hline $\mathrm{C}$ & -0.46762 & -1.57053 & 2.17098 & $\mathrm{O}$ & 1.67026 & 4.52156 & 1.30859 \\
\hline $\mathrm{C}$ & -1.76714 & -1.73944 & 2.91795 & $\mathrm{C}$ & -0.09468 & 3.1455 & 0.49132 \\
\hline $\mathrm{H}$ & -2.53795 & -1.0754 & 2.50077 & $\mathrm{C}$ & -0.47405 & 2.07371 & -0.29854 \\
\hline $\mathrm{H}$ & -2.15485 & -2.76059 & 2.89673 & $\mathrm{C}$ & -1.82248 & 1.60299 & -0.41041 \\
\hline $\mathrm{H}$ & -1.64916 & -1.44896 & 3.97053 & $\mathrm{C}$ & -2.17845 & 0.5106 & -1.15537 \\
\hline $\mathrm{C}$ & 0.11293 & -0.18126 & 2.26001 & $\mathrm{C}$ & -3.5754 & 0.05872 & -1.21332 \\
\hline $\mathrm{H}$ & -0.62779 & 0.57772 & 1.98327 & $\mathrm{C}$ & -4.35451 & -0.01649 & -0.1262 \\
\hline $\mathrm{H}$ & 0.42255 & 0.05033 & 3.2886 & $\mathrm{C}$ & -5.80434 & -0.40264 & -0.11364 \\
\hline $\mathrm{H}$ & 0.98345 & -0.05682 & 1.61067 & $\mathrm{C}$ & -6.01659 & -1.64475 & 0.76921 \\
\hline $\mathrm{H}$ & 1.09405 & -2.28941 & 0.99211 & $\mathrm{H}$ & -7.07566 & -1.92412 & 0.79273 \\
\hline $\mathrm{H}$ & -1.19616 & -4.18851 & 1.8244 & $\mathrm{H}$ & -5.44378 & -2.50073 & 0.39876 \\
\hline $\mathrm{H}$ & 0.50349 & -4.63313 & 1.76164 & $\mathrm{H}$ & -5.69853 & -1.44801 & 1.80033 \\
\hline $\mathrm{H}$ & 0.62961 & -4.35896 & -0.62953 & $\mathrm{C}$ & -6.67401 & 0.77575 & 0.37936 \\
\hline $\mathrm{H}$ & -0.75353 & -5.388 & -0.25486 & $\mathrm{C}$ & -6.60381 & 2.01219 & -0.51846 \\
\hline $\mathrm{H}$ & 0.3146 & -2.66046 & -2.06146 & $\mathrm{H}$ & -5.58688 & 2.41586 & -0.5667 \\
\hline $\mathrm{N}$ & -1.21094 & -0.16452 & -1.89124 & $\mathrm{H}$ & -6.91264 & 1.7717 & -1.54313 \\
\hline $\mathrm{C}$ & 0.08712 & 0.22844 & -1.80218 & $\mathrm{H}$ & -7.26262 & 2.8043 & -0.14788 \\
\hline $\mathrm{C}$ & 0.50625 & 1.28792 & -1.04135 & $\mathrm{H}$ & -6.36796 & 1.03798 & 1.40183 \\
\hline $\mathrm{C}$ & 1.89662 & 1.62986 & -0.84453 & $\mathrm{H}$ & -7.712 & 0.4258 & 0.44994 \\
\hline $\mathrm{C}$ & 3.04023 & 0.90321 & -0.67498 & $\mathrm{H}$ & -6.10986 & -0.64377 & -1.14189 \\
\hline $\mathrm{C}$ & 3.20805 & -0.55824 & -0.7592 & $\mathrm{H}$ & -3.91703 & 0.21647 & 0.84603 \\
\hline $\mathrm{O}$ & 2.49665 & -1.21826 & -1.52718 & $\mathrm{H}$ & -3.99057 & -0.19571 & -2.18693 \\
\hline $\mathrm{C}$ & 4.23875 & -1.22978 & 0.13598 & $\mathrm{H}$ & -2.60605 & 2.14768 & 0.09692 \\
\hline $\mathrm{C}$ & 4.39172 & -2.71979 & -0.24156 & $\mathrm{Cl}$ & -1.2705 & 3.94878 & 1.51827 \\
\hline $\mathrm{O}$ & 3.16113 & -3.42884 & -0.0803 & $\mathrm{C}$ & 2.05922 & 3.96745 & -1.77987 \\
\hline $\mathrm{H}$ & 2.55986 & -2.99506 & -0.71091 & $\mathrm{H}$ & 2.73763 & 3.6179 & -2.5637 \\
\hline $\mathrm{C}$ & 5.44312 & -3.44147 & 0.58776 & $\mathrm{H}$ & 1.03268 & 3.95376 & -2.15519 \\
\hline $\mathrm{H}$ & 6.40543 & -2.92013 & 0.54058 & $\mathrm{H}$ & 2.32551 & 4.98909 & -1.49767 \\
\hline $\mathrm{H}$ & 5.57644 & $\begin{array}{l}-4.45919 \\
\end{array}$ & 0.20919 & $\mathrm{H}$ & 0.80786 & -0.36496 & -2.34246 \\
\hline $\mathrm{H}$ & 5.13184 & -3.51018 & 1.6342 & $\mathrm{H}$ & -2.53525 & -1.62584 & -2.60568 \\
\hline $\mathrm{H}$ & 4.694 & -2.75606 & -1.30025 & $\mathrm{H}$ & -1.1026 & -1.36672 & -3.59754 \\
\hline
\end{tabular}

Table S19. Optimized Z-matrixes of Conf2-7 in the gas phase $(\AA)$ at the B3LYP/6-31G(d) level of theory.

\begin{tabular}{|l|r|r|r|r|r|r|r|}
\hline $\mathrm{C}$ & -1.93099 & 1.12671 & 1.04491 & $\mathrm{C}$ & 3.8726 & 0.57254 & -2.12811 \\
\hline $\mathrm{C}$ & -1.67124 & 2.081 & -0.08438 & $\mathrm{H}$ & 4.6829 & 0.86891 & -2.7989 \\
\hline
\end{tabular}




\begin{tabular}{|c|c|c|c|c|c|c|c|}
\hline $\mathrm{C}$ & -2.50983 & 2.98611 & -0.60532 & $\mathrm{H}$ & 2.92974 & 0.93617 & -2.54625 \\
\hline $\mathrm{C}$ & -3.93214 & 3.19825 & -0.15513 & $\mathrm{H}$ & 3.85368 & -0.52003 & -2.09728 \\
\hline $\mathrm{H}$ & -4.61888 & 3.1293 & -1.00859 & $\mathrm{H}$ & 5.07623 & 0.8294 & -0.36532 \\
\hline $\mathrm{H}$ & -4.26307 & 2.48553 & 0.60477 & $\mathrm{C}$ & 4.34279 & -1.66361 & 0.50223 \\
\hline $\mathrm{H}$ & -4.05773 & 4.20563 & 0.26355 & $\mathrm{O}$ & 5.48709 & -1.33034 & 0.30551 \\
\hline $\mathrm{C}$ & -1.99945 & 3.95153 & -1.65022 & $\mathrm{O}$ & 3.99155 & -2.96833 & 0.72125 \\
\hline $\mathrm{C}$ & -1.4213 & 5.24853 & -1.01294 & $\mathrm{C}$ & 2.58284 & -3.06789 & 0.9407 \\
\hline $\mathrm{C}$ & -0.11492 & 5.02022 & -0.30311 & $\mathrm{C}$ & 1.92955 & -4.09312 & -0.03272 \\
\hline $\mathrm{C}$ & 0.08225 & 4.84678 & 1.01307 & $\mathrm{O}$ & 2.56088 & -5.07304 & -0.38892 \\
\hline $\mathrm{C}$ & -1.01156 & 4.8613 & 2.04992 & $\mathrm{C}$ & 0.53413 & -3.82306 & -0.31484 \\
\hline $\mathrm{H}$ & -1.02915 & 3.90976 & 2.60001 & $\mathrm{C}$ & -0.10424 & -2.6416 & 0.0234 \\
\hline $\mathrm{H}$ & -0.8287 & 5.64469 & 2.79805 & $\mathrm{C}$ & -1.4785 & -2.35603 & -0.27988 \\
\hline $\mathrm{H}$ & -2.00653 & 5.01858 & 1.62894 & $\mathrm{C}$ & -2.08293 & -1.1717 & 0.04224 \\
\hline $\mathrm{C}$ & 1.46759 & 4.61954 & 1.5663 & $\mathrm{C}$ & -3.46019 & -0.84803 & -0.34764 \\
\hline $\mathrm{H}$ & 1.71207 & 5.37079 & 2.32989 & $\mathrm{C}$ & -4.48499 & -1.70611 & -0.26645 \\
\hline $\mathrm{H}$ & 1.54262 & 3.6371 & 2.052 & $\mathrm{C}$ & -5.88948 & -1.40389 & -0.70293 \\
\hline $\mathrm{H}$ & 2.22499 & 4.66233 & 0.78013 & $\mathrm{C}$ & -6.36906 & -2.45412 & -1.71908 \\
\hline $\mathrm{H}$ & 0.75926 & 4.9618 & -0.95215 & $\mathrm{H}$ & -7.38672 & -2.22773 & -2.05589 \\
\hline $\mathrm{H}$ & -1.26687 & 5.97603 & -1.81977 & $\mathrm{H}$ & -5.71539 & -2.48482 & -2.59677 \\
\hline $\mathrm{H}$ & -2.17161 & 5.68628 & -0.34547 & $\mathrm{H}$ & -6.37886 & -3.45516 & -1.27106 \\
\hline $\mathrm{H}$ & -2.81135 & 4.22445 & -2.33638 & $\mathrm{C}$ & -6.83927 & -1.33576 & 0.51355 \\
\hline $\mathrm{H}$ & -1.2139 & 3.47803 & -2.24972 & $\mathrm{C}$ & -6.49694 & -0.21451 & 1.49676 \\
\hline $\mathrm{H}$ & -0.65403 & 2.03684 & -0.46873 & $\mathrm{H}$ & -7.21521 & -0.17891 & 2.32253 \\
\hline $\mathrm{N}$ & -1.36442 & -0.20286 & 0.73736 & $\mathrm{H}$ & -5.49813 & -0.35294 & 1.92505 \\
\hline $\mathrm{C}$ & -0.04447 & -0.38943 & 0.98282 & $\mathrm{H}$ & -6.51251 & 0.76302 & 0.99816 \\
\hline $\mathrm{C}$ & 0.62429 & -1.53487 & 0.63913 & $\mathrm{H}$ & -6.82584 & -2.30462 & 1.03198 \\
\hline $\mathrm{C}$ & 2.05482 & -1.67698 & 0.75961 & $\mathrm{H}$ & -7.86272 & -1.20071 & 0.14046 \\
\hline $\mathrm{C}$ & 3.10669 & -0.83141 & 0.55237 & $\mathrm{H}$ & -5.89382 & -0.41779 & -1.18889 \\
\hline $\mathrm{C}$ & 3.05818 & 0.60486 & 0.22659 & $\mathrm{H}$ & -4.31737 & -2.69456 & 0.16479 \\
\hline $\mathrm{O}$ & 2.15077 & 1.31632 & 0.67631 & $\mathrm{H}$ & -3.62768 & 0.15153 & -0.74398 \\
\hline $\mathrm{C}$ & 4.09849 & 1.17256 & -0.72394 & $\mathrm{H}$ & -2.05025 & -3.0833 & -0.8395 \\
\hline $\mathrm{C}$ & 4.05844 & 2.71655 & -0.71259 & $\mathrm{Cl}$ & -0.32971 & -5.08297 & -1.17933 \\
\hline $\mathrm{O}$ & 2.81487 & 3.21307 & -1.21171 & $\mathrm{C}$ & 2.35309 & -3.5865 & 2.37145 \\
\hline $\mathrm{H}$ & 2.14669 & 2.85177 & -0.60235 & $\mathrm{H}$ & 2.8345 & -2.91438 & 3.08792 \\
\hline $\mathrm{C}$ & 5.16335 & 3.34682 & -1.54732 & $\mathrm{H}$ & 1.28381 & -3.64627 & 2.59108 \\
\hline $\mathrm{H}$ & 6.14788 & 2.98993 & -1.22638 & $\mathrm{H}$ & 2.79394 & -4.58313 & 2.45285 \\
\hline $\mathrm{H}$ & 5.13209 & 4.43499 & -1.43737 & $\mathrm{H}$ & 0.4794 & 0.43028 & 1.44951 \\
\hline $\mathrm{H}$ & 5.03321 & 3.1131 & -2.6082 & $\mathrm{H}$ & -2.9883 & 1.00567 & 1.27929 \\
\hline $\mathrm{H}$ & 4.18447 & 3.03733 & 0.3338 & $\mathrm{H}$ & -1.43378 & 1.46605 & 1.96102 \\
\hline
\end{tabular}

\section{Computational data of compound 1}

Table S20. Experimental and calculated ${ }^{13} \mathrm{C}-\mathrm{NMR}$ chemical shifts of Conf1 and Conf2

No.

$1-\delta_{\text {exptl }}$
Conf1- $\delta_{\text {calcd }}$. 


\begin{tabular}{|c|c|c|c|}
\hline 1 & 141.2 & 143.2 & 142.1 \\
\hline 3 & 147.5 & 145.7 & 146.9 \\
\hline 4 & 111.2 & 109.7 & 109.7 \\
\hline $4 a$ & 145.0 & 143.0 & 143.1 \\
\hline 5 & 99.8 & 107.0 & 107.7 \\
\hline 6 & 181.5 & 177.1 & 177.3 \\
\hline 7 & 88.9 & 90.4 & 90.7 \\
\hline 8 & 169.2 & 172.1 & 173.6 \\
\hline $8 a$ & 111.5 & 110.5 & 110.0 \\
\hline 9 & 119.4 & 120.8 & 121.5 \\
\hline 10 & 149.0 & 150.4 & 150.8 \\
\hline 11 & 39.3 & 43.9 & 44.4 \\
\hline 12 & 29.1 & 31.8 & 31.8 \\
\hline 13 & 11.8 & 14.6 & 14.7 \\
\hline 14 & 19.4 & 22.4 & 22.4 \\
\hline 15 & 27.6 & 29.2 & 29.2 \\
\hline $1^{\prime}$ & 168.9 & 169.8 & 167.2 \\
\hline $2^{\prime}$ & 122.7 & 120.5 & 119.3 \\
\hline $3^{\prime}$ & 201.5 & 201.1 & 202.5 \\
\hline $4^{\prime}$ & 50.7 & 53.5 & 49.7 \\
\hline $5^{\prime}$ & 70.7 & 73.0 & 70.0 \\
\hline $6^{\prime}$ & 21.3 & 23.6 & 19.5 \\
\hline $7^{\prime}$ & 13.7 & 16.0 & 13.4 \\
\hline $1 "$ & 52.5 & 53.0 & 53.2 \\
\hline $2^{\prime \prime}$ & 116.4 & 115.7 & 118.0 \\
\hline 3" & 144.5 & 146.7 & 144.2 \\
\hline 4" & 39.5 & 40.7 & 40.0 \\
\hline $5 "$ & 26.1 & 30.9 & 29.5 \\
\hline $6 "$ & 123.2 & 122.5 & 123.8 \\
\hline 7" & 132.4 & 135.7 & 133.7 \\
\hline $8^{\prime \prime}$ & 25.7 & 26.9 & 27.4 \\
\hline $9^{\prime \prime}$ & 17.8 & 20.0 & 19.3 \\
\hline $10^{\prime \prime}$ & 16.9 & 21.1 & 20.1 \\
\hline
\end{tabular}

Table S21. Experimental and calculated ${ }^{1} \mathrm{H}-\mathrm{NMR}$ chemical shifts of Conf1 and Conf2

\begin{tabular}{|c|c|c|c|}
\hline No. & $\mathbf{1}-\delta_{\text {exptl. }}$ & Conf1- $\delta_{\text {calcd. }}$ & Conf2- $\delta_{\text {calcd. }}$ \\
\hline 1 & 8.78 & 10.28 & 9.80 \\
\hline 4 & 6.88 & 6.80 & 6.76 \\
\hline 9 & 6.22 & 6.62 & 6.55 \\
\hline 10 & 6.40 & 6.62 & 6.69 \\
\hline 11 & 2.29 & 2.17 & 2.16 \\
\hline 12 & 1.47 & 1.32 & 1.31 \\
\hline 13 & 0.94 & 0.69 & 0.76 \\
\hline
\end{tabular}




\begin{tabular}{|l|l|l|l|}
\hline 14 & 1.12 & 1.00 & 1.01 \\
\hline 15 & 1.71 & 1.63 & 1.65 \\
\hline $4^{\prime}$ & 3.71 & 3.98 & 4.00 \\
\hline $5^{\prime}$ & 3.86 & 3.54 & 4.08 \\
\hline $6^{\prime}$ & 1.13 & 1.07 & 1.07 \\
\hline $7^{\prime}$ & 1.17 & 0.96 & 0.94 \\
\hline $1^{\prime \prime}$ & 4.50 & 4.37 & 4.34 \\
\hline $2^{\prime \prime}$ & 5.29 & 5.63 & 5.64 \\
\hline $4^{\prime \prime}$ & 2.16 & 1.91 & 1.98 \\
\hline $5^{\prime \prime}$ & 2.16 & 1.90 & 1.95 \\
\hline $8^{\prime \prime}$ & 5.07 & 5.02 & 4.93 \\
\hline $9^{\prime \prime}$ & 1.68 & 1.36 & 1.09 \\
\hline $1^{\prime \prime}$ & 1.60 & 0.81 & 0.98 \\
\hline
\end{tabular}
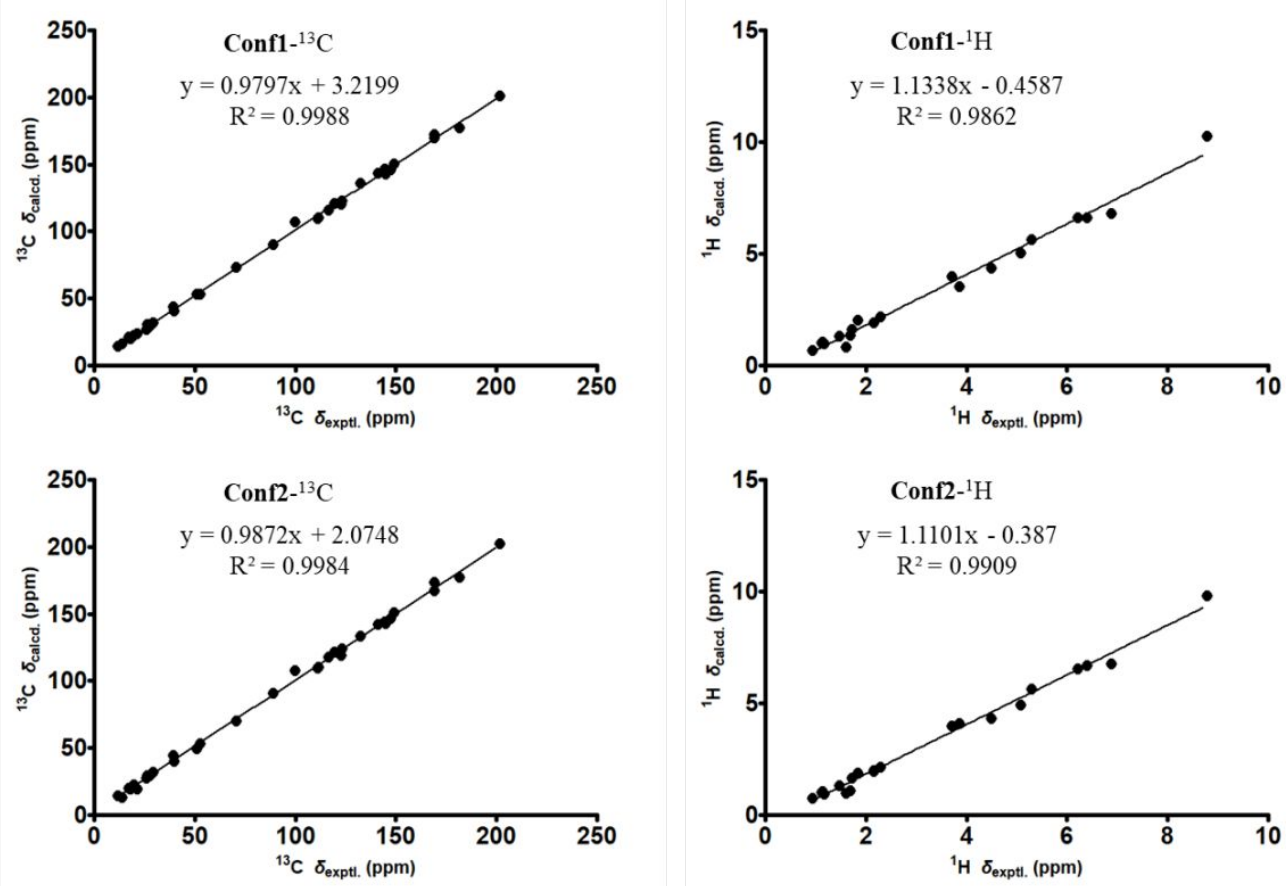

Figure S3. Linear regression analysis between the exptl. and calcd. NMR data of Conf1 and Conf2 


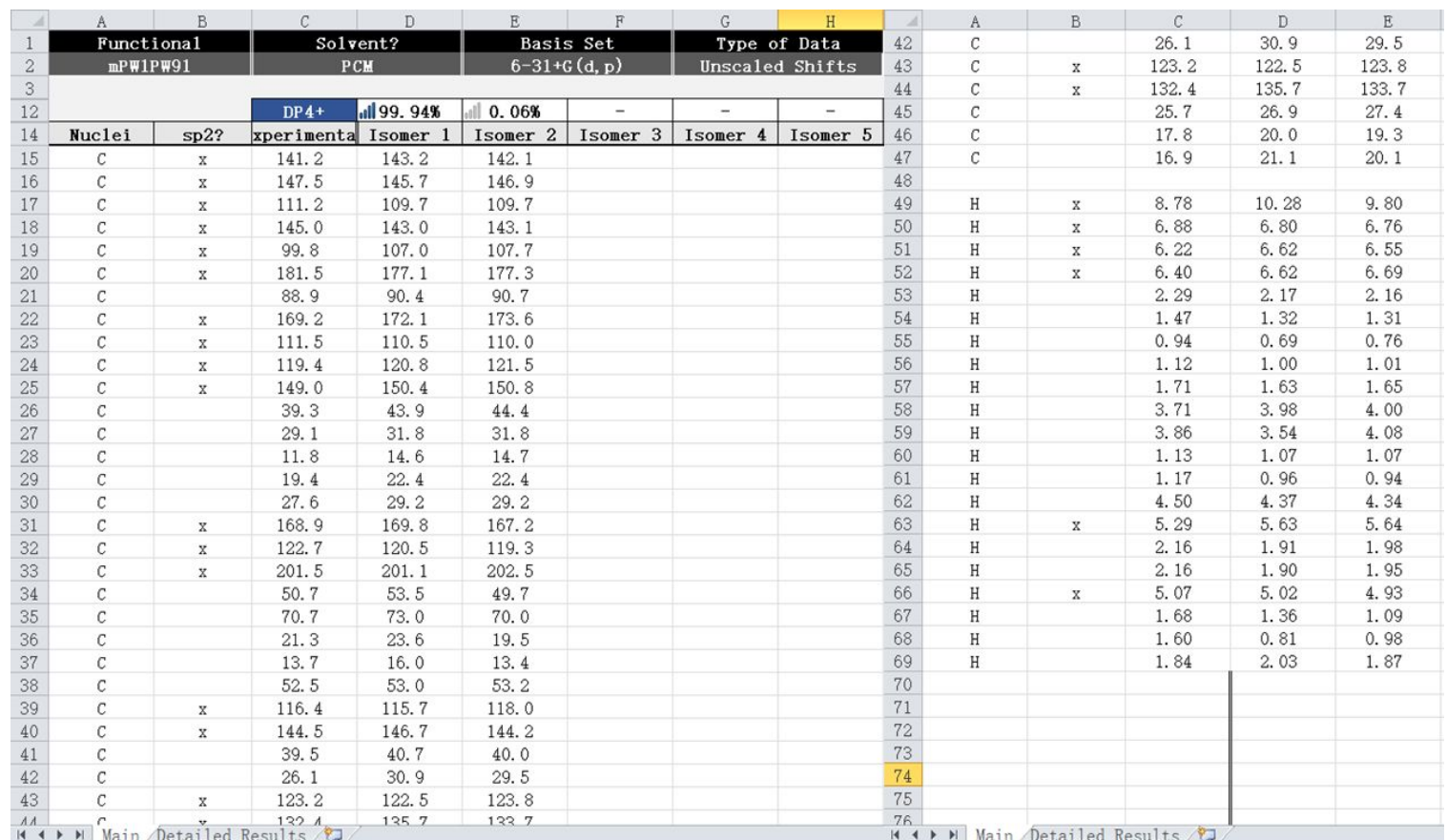

Figure S4. Detailed DP4+ probability (calculated at mPW1PW91/6-31+G(d,p) level) for compound 1. Isomer 1 is Conf1, isomer 2 is Conf2.

\section{Computational data of compound 2}

Table S22. Experimental and calculated ${ }^{13} \mathrm{C}-\mathrm{NMR}$ chemical shifts of Conf1 and Conf2

\begin{tabular}{|c|c|c|c|}
\hline No. & $\mathbf{1}-\delta_{\text {expt. }}$ & Conf1- $\delta_{\text {calcd. }}$ & Conf2- $\delta_{\text {calcd. }}$ \\
\hline 1 & 141.4 & 143.2 & 142.1 \\
\hline 3 & 147.6 & 145.7 & 146.9 \\
\hline 4 & 111.2 & 109.7 & 109.7 \\
\hline $4 a$ & 145.1 & 143.0 & 143.1 \\
\hline 5 & 99.8 & 107.0 & 107.7 \\
\hline 6 & 181.3 & 177.1 & 177.3 \\
\hline 7 & 89.1 & 90.4 & 90.7 \\
\hline 8 & 171.0 & 172.1 & 173.6 \\
\hline $8 \mathrm{a}$ & 111.4 & 110.5 & 110.0 \\
\hline 9 & 119.4 & 120.8 & 121.5 \\
\hline 10 & 149.2 & 150.4 & 150.8 \\
\hline 11 & 39.4 & 43.9 & 44.4 \\
\hline 12 & 29.1 & 31.8 & 31.8 \\
\hline 13 & 11.8 & 14.6 & 14.7 \\
\hline 14 & 19.4 & 22.4 & 22.4 \\
\hline 15 & 27.4 & 29.2 & 29.2 \\
\hline $1^{\prime}$ & 169.0 & 169.8 & 167.2 \\
\hline $2^{\prime}$ & 121.3 & 120.5 & 119.3 \\
\hline $3^{\prime}$ & 201.6 & 201.1 & 202.5 \\
\hline 4 & 48.2 & 53.5 & 49.7 \\
\hline
\end{tabular}




\begin{tabular}{|c|c|c|c|}
\hline $5^{\prime}$ & 67.6 & 73.0 & 70.0 \\
\hline $6^{\prime}$ & 19.3 & 23.6 & 19.5 \\
\hline $7^{\prime}$ & 10.1 & 16.0 & 13.4 \\
\hline $1^{\prime \prime}$ & 52.5 & 53.0 & 53.2 \\
\hline $2^{\prime \prime}$ & 116.3 & 115.7 & 118.0 \\
\hline $3^{\prime \prime}$ & 144.7 & 146.7 & 144.2 \\
\hline $4^{\prime \prime}$ & 39.5 & 40.7 & 40.0 \\
\hline $5^{\prime \prime}$ & 26.1 & 30.9 & 29.5 \\
\hline $6^{\prime \prime}$ & 123.1 & 122.5 & 123.8 \\
\hline $7^{\prime \prime}$ & 132.4 & 135.7 & 133.7 \\
\hline $8^{\prime \prime}$ & 25.7 & 26.9 & 27.4 \\
\hline $9^{\prime \prime}$ & 17.8 & 20.0 & 19.3 \\
\hline $10^{\prime \prime}$ & 16.9 & 21.1 & 20.1 \\
\hline
\end{tabular}

Table S23. Experimental and calculated 1H-NMR chemical shifts of Conf1 and Conf2

\begin{tabular}{|c|c|c|c|}
\hline No. & $\mathbf{1 -} \delta_{\text {exptl. }}$ & Conf1- $\delta_{\text {calcd. }}$ & Conf2- calcd. $_{\text {. }}$ \\
\hline 1 & 8.79 & 10.28 & 9.80 \\
\hline 4 & 6.89 & 6.80 & 6.76 \\
\hline 9 & 6.24 & 6.62 & 6.55 \\
\hline 10 & 6.41 & 6.62 & 2.16 \\
\hline 11 & 2.30 & 2.17 & 1.31 \\
\hline 12 & 1.48 & 1.32 & 0.76 \\
\hline 13 & 0.94 & 0.69 & 1.01 \\
\hline 14 & 1.12 & 1.00 & 1.65 \\
\hline 15 & 1.72 & 1.63 & 4.00 \\
\hline $4^{\prime}$ & 3.79 & 3.98 & 4.08 \\
\hline $5^{\prime}$ & 4.27 & 3.54 & 1.07 \\
\hline $6^{\prime}$ & 1.22 & 1.07 & 0.94 \\
\hline $7^{\prime}$ & 1.02 & 0.96 & 4.34 \\
\hline $1^{\prime \prime}$ & 4.52 & 4.37 & 5.64 \\
\hline $2^{\prime \prime}$ & 5.30 & 5.63 & 1.98 \\
\hline $4^{\prime \prime}$ & 2.16 & 1.91 & 1.95 \\
\hline $5^{\prime \prime}$ & 2.16 & 1.90 & 4.93 \\
\hline $6^{\prime \prime}$ & 5.06 & 5.02 & 1.09 \\
\hline $8^{\prime \prime}$ & 1.67 & 1.36 & 0.98 \\
\hline $9^{\prime \prime}$ & 1.60 & 0.81 & 1.87 \\
\hline $10^{\prime \prime}$ & 1.84 & 2.03 & \\
\hline
\end{tabular}



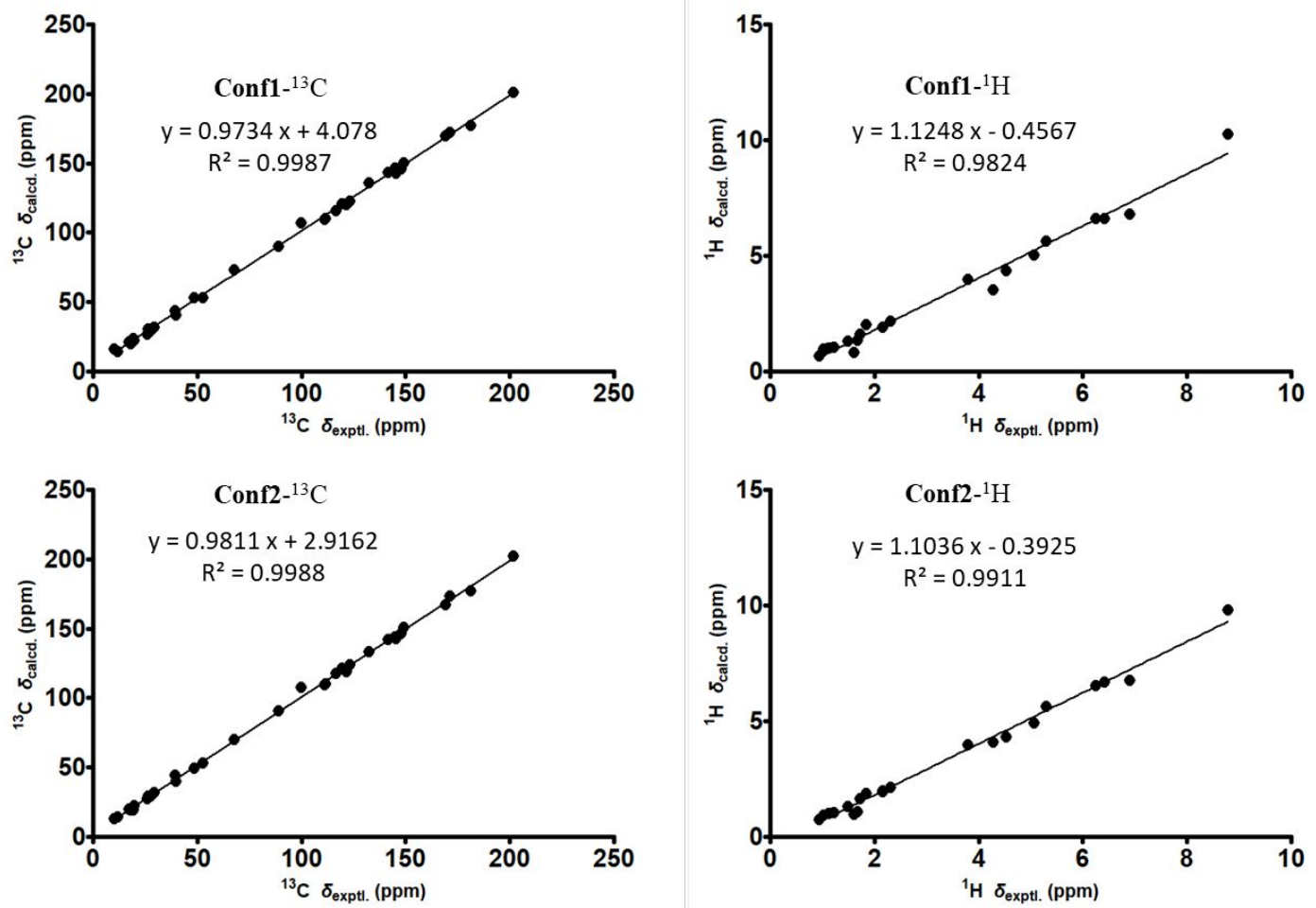

Figure S5. Linear regression analysis between the exptl. and calcd. NMR data of Conf1 and Conf2.

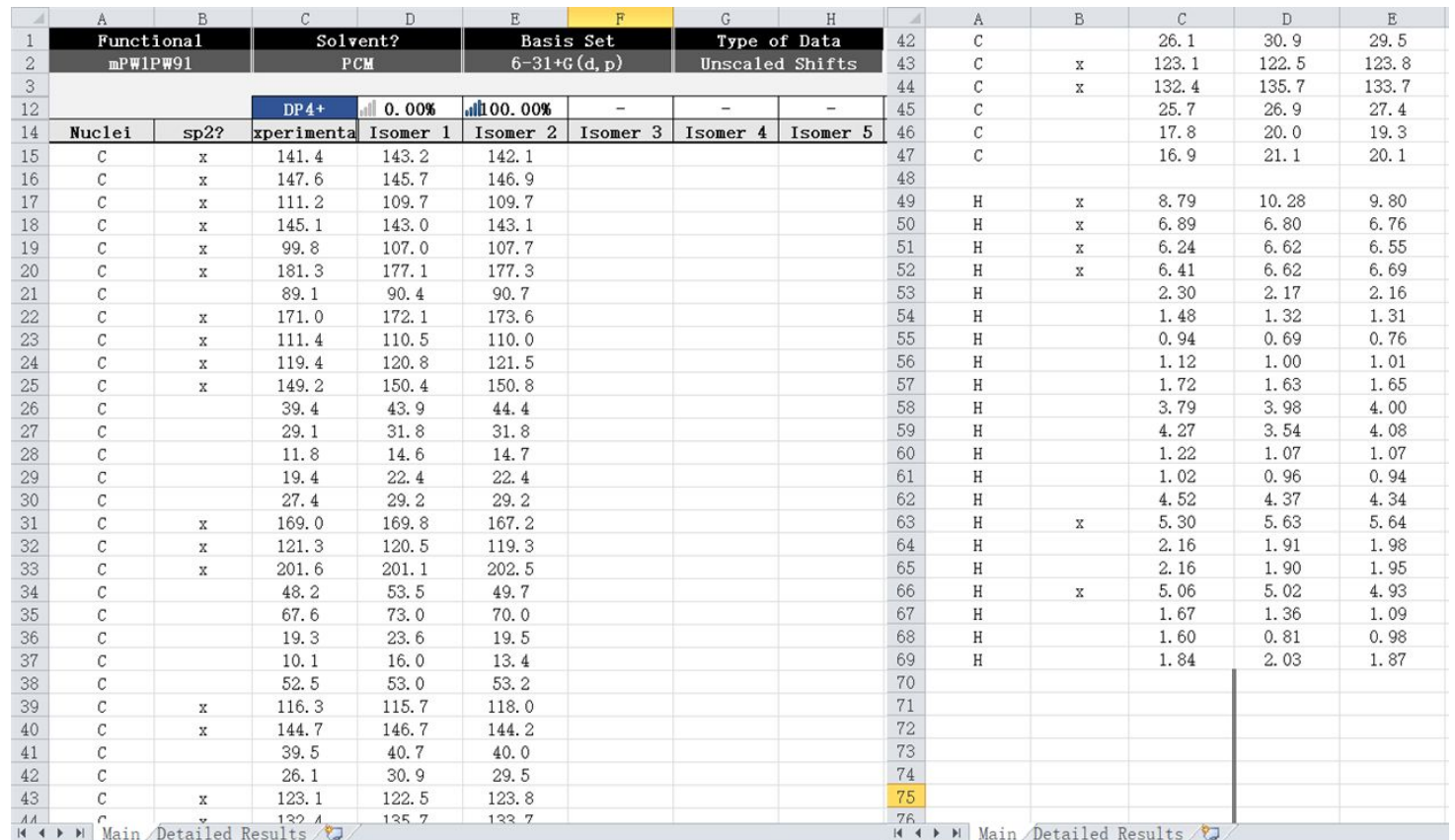

Figure S6. Detailed DP4+ probability (calculated at mPW1PW91/6-31+G(d,p) level) for compound 2. Isomer 1 is Conf1, isomer 2 is Conf2. 


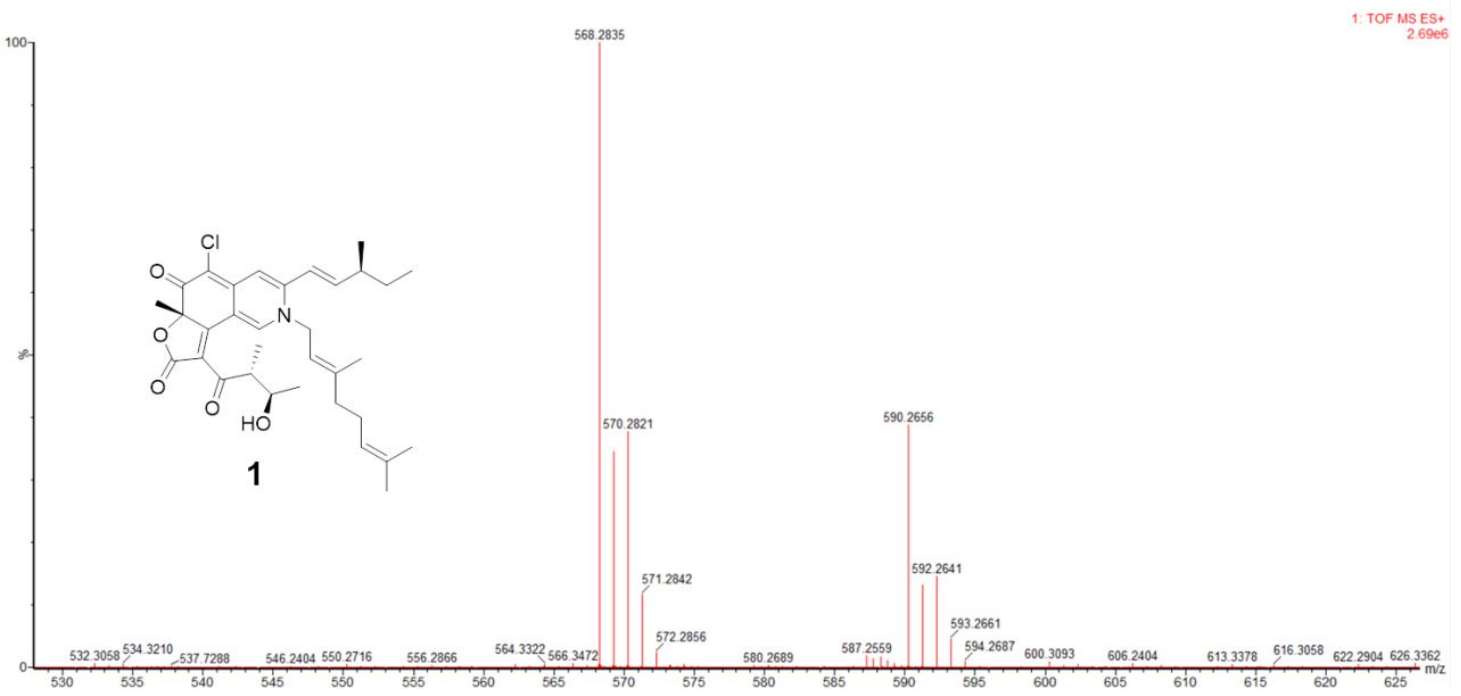

Figure S7. HRESIMS spectrum of compound 1

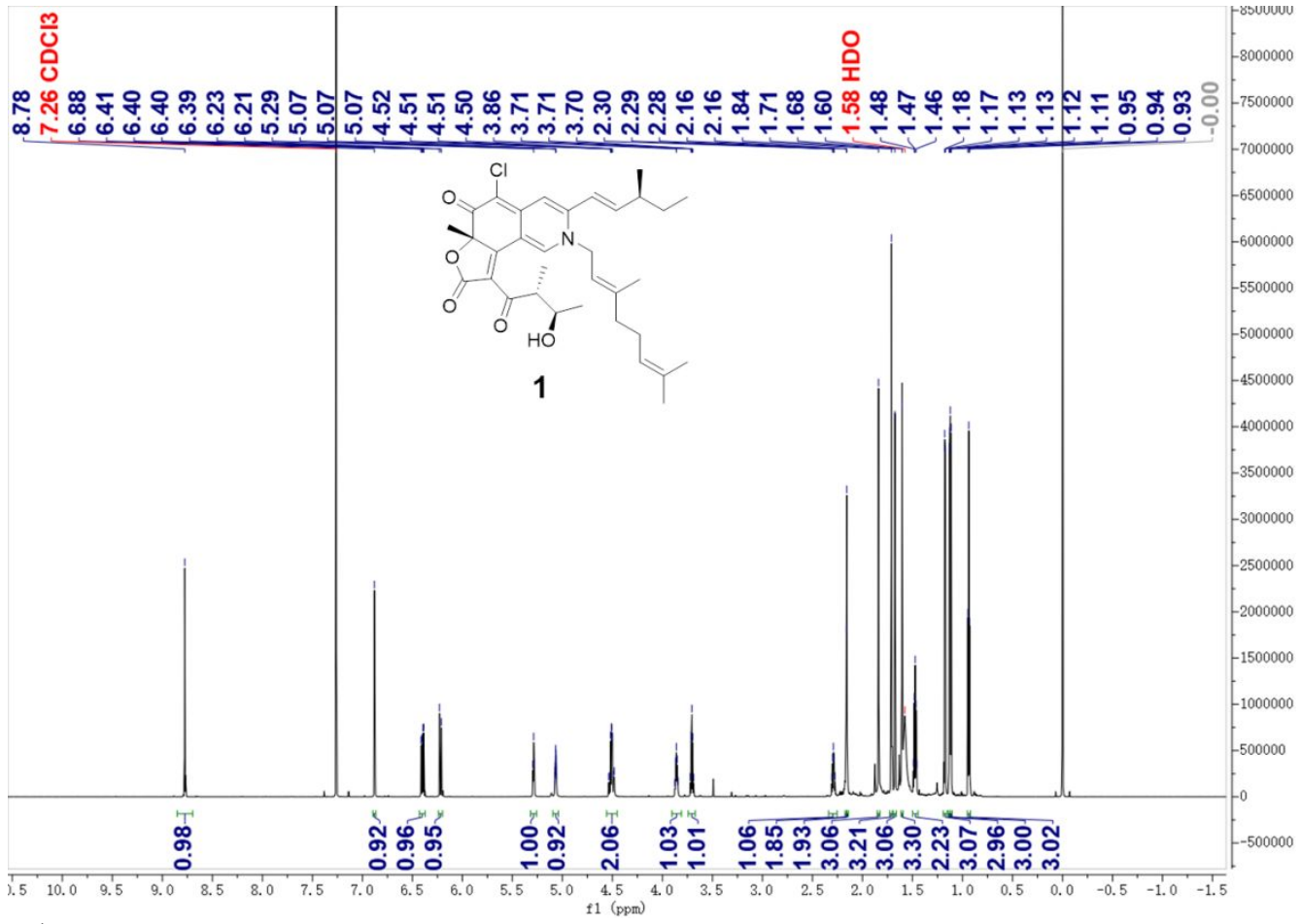

Figure S8. ${ }^{1} \mathrm{H}$ NMR spectrum of compound 1 


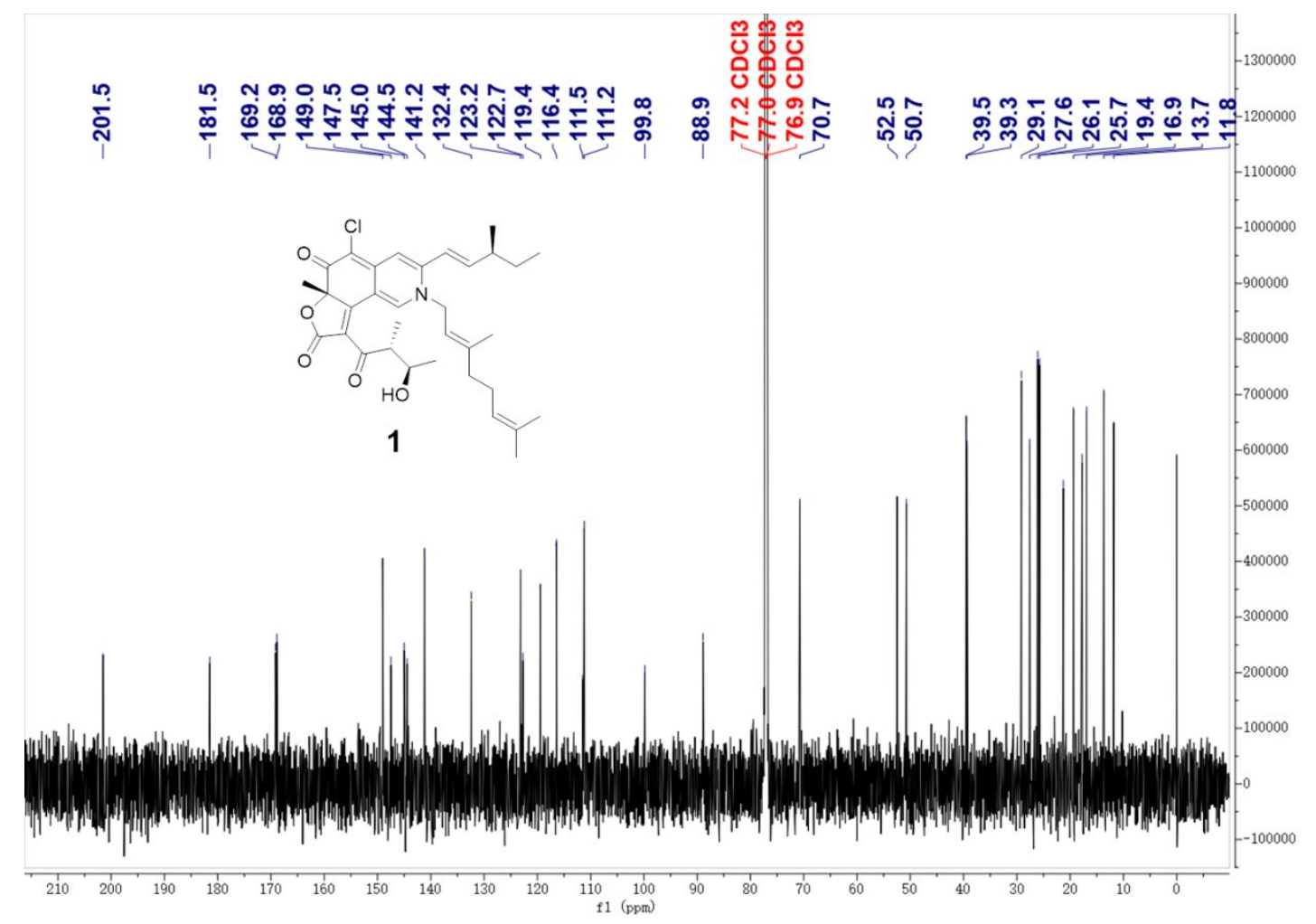

Figure S9. ${ }^{13} \mathrm{C}$ NMR spectrum of compound 1

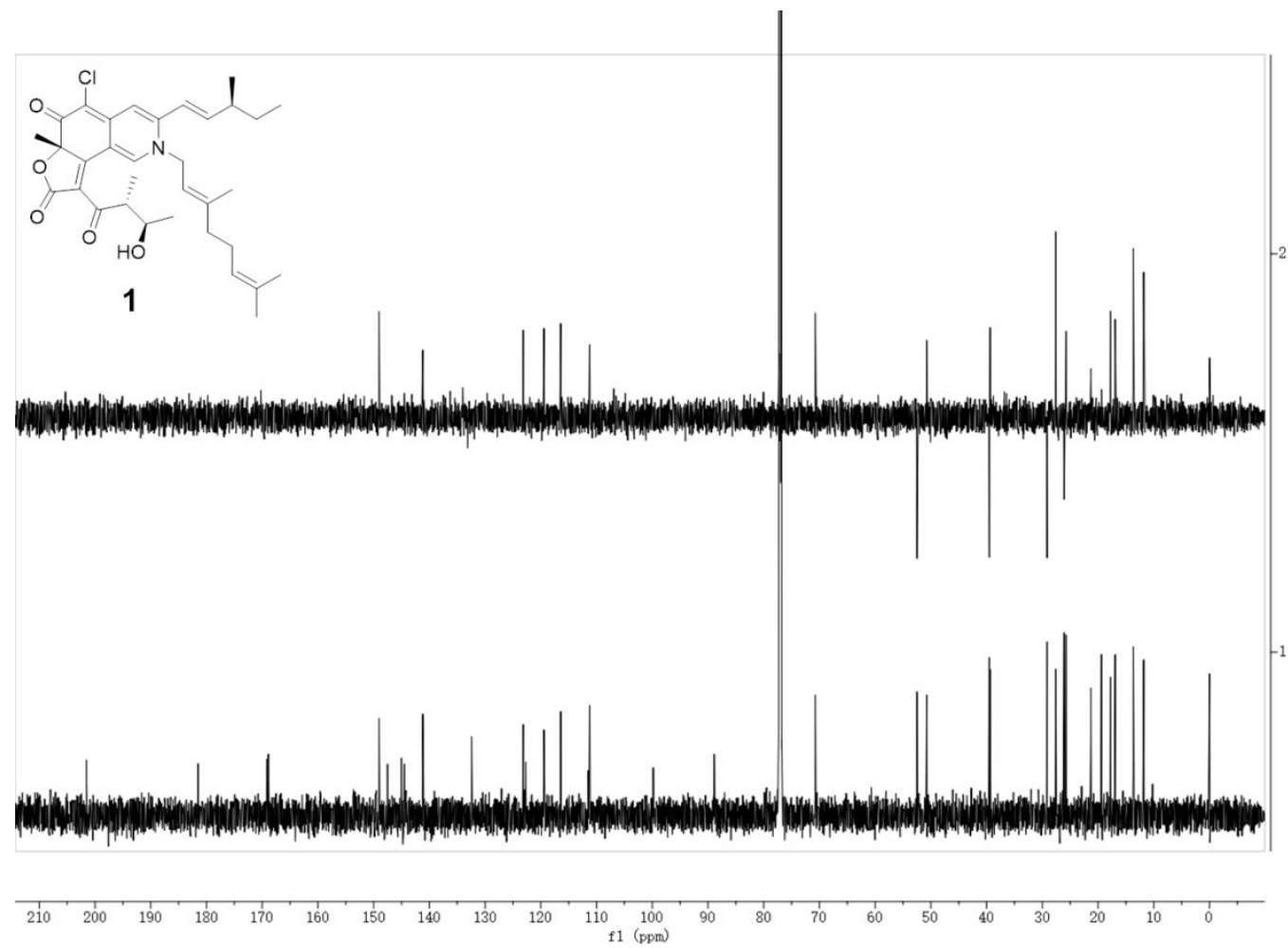

Figure S10. ${ }^{13} \mathrm{C} / \mathrm{DEPT}$ spectrum of compound $\mathbf{1}$ 


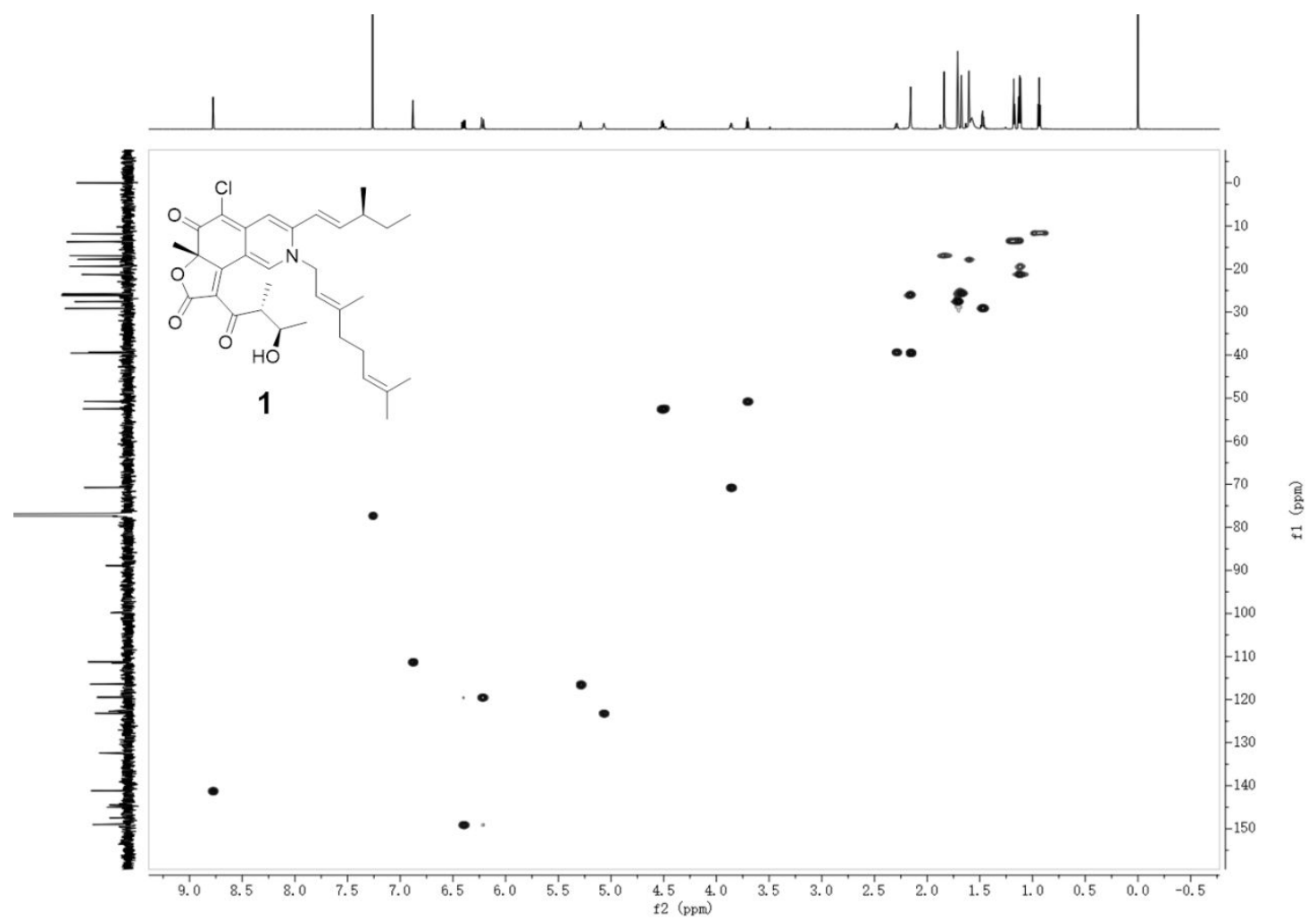

Figure S11. HSQC spectrum of compound 1

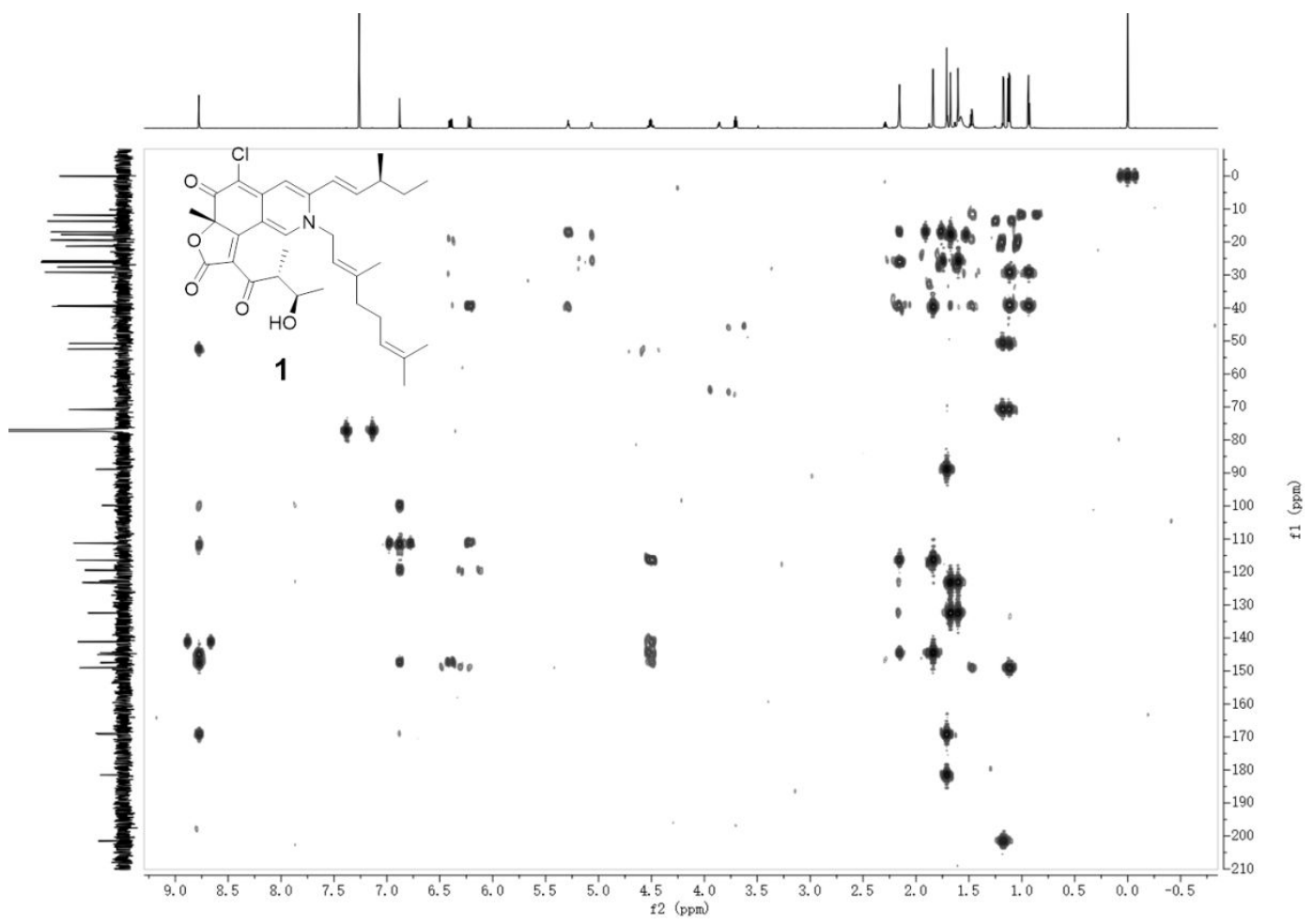

Figure S12. HMBC spectrum of compound 1 


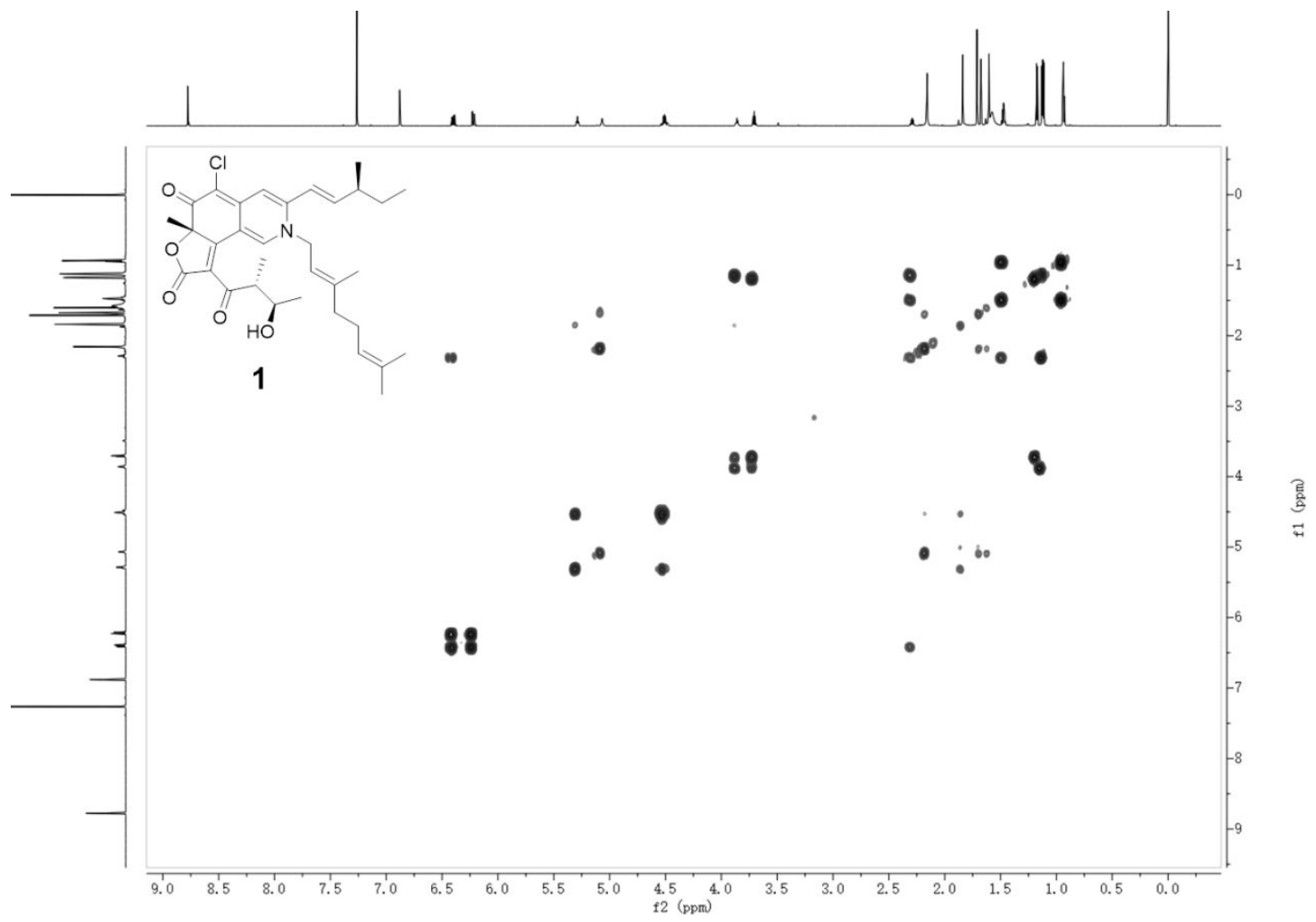

Figure S13. COSY spectrum of compound 1

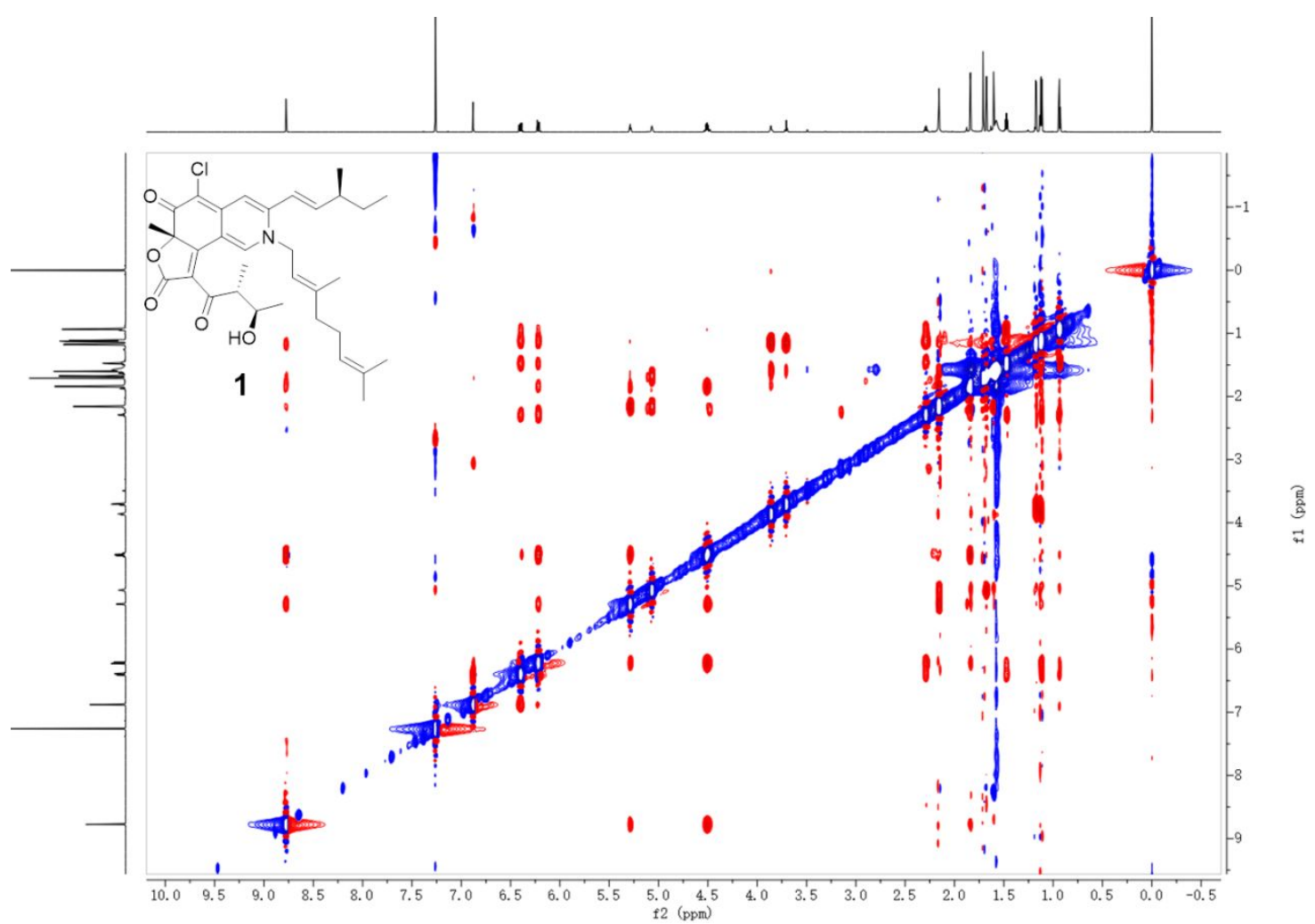

Figure S14. NOESY spectrum of compound 1 


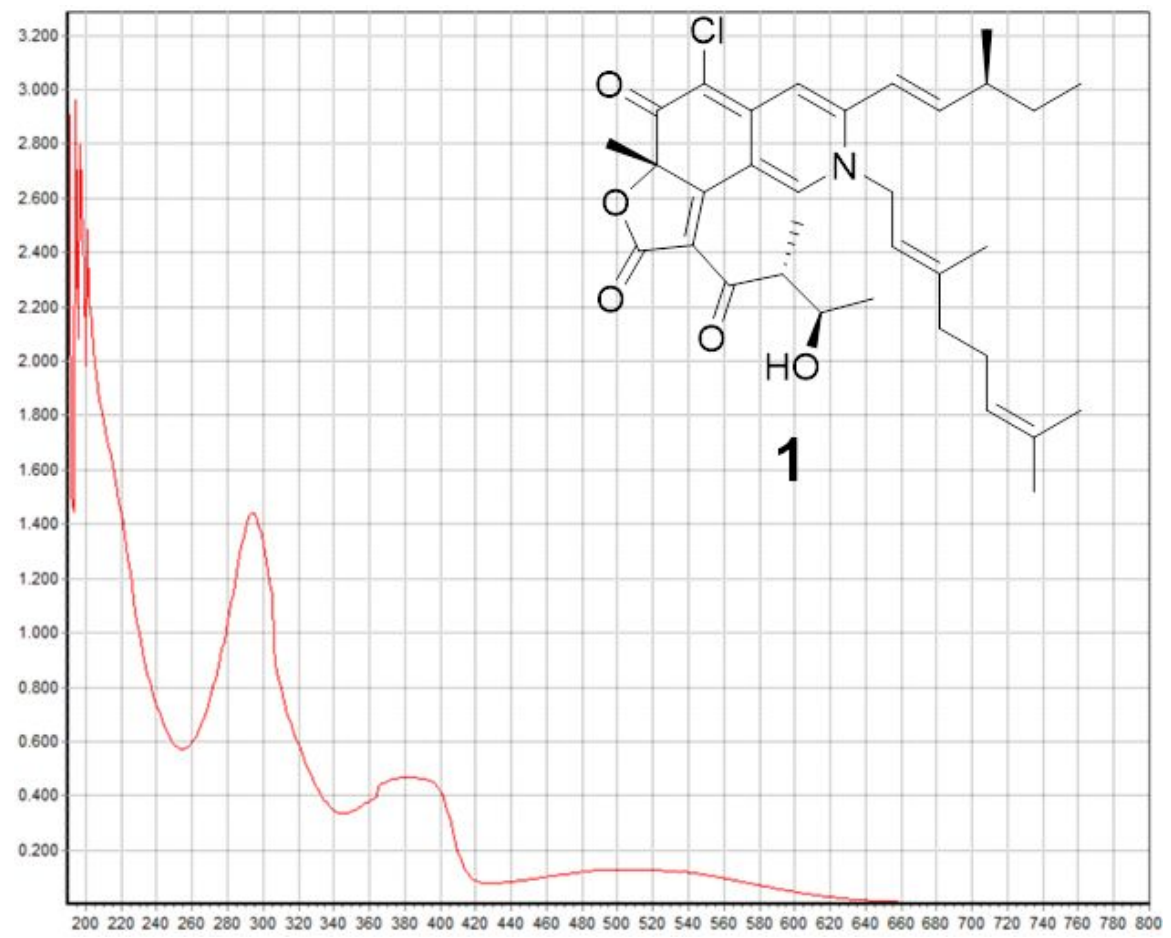

Figure S15. UV spectrum of compound 1

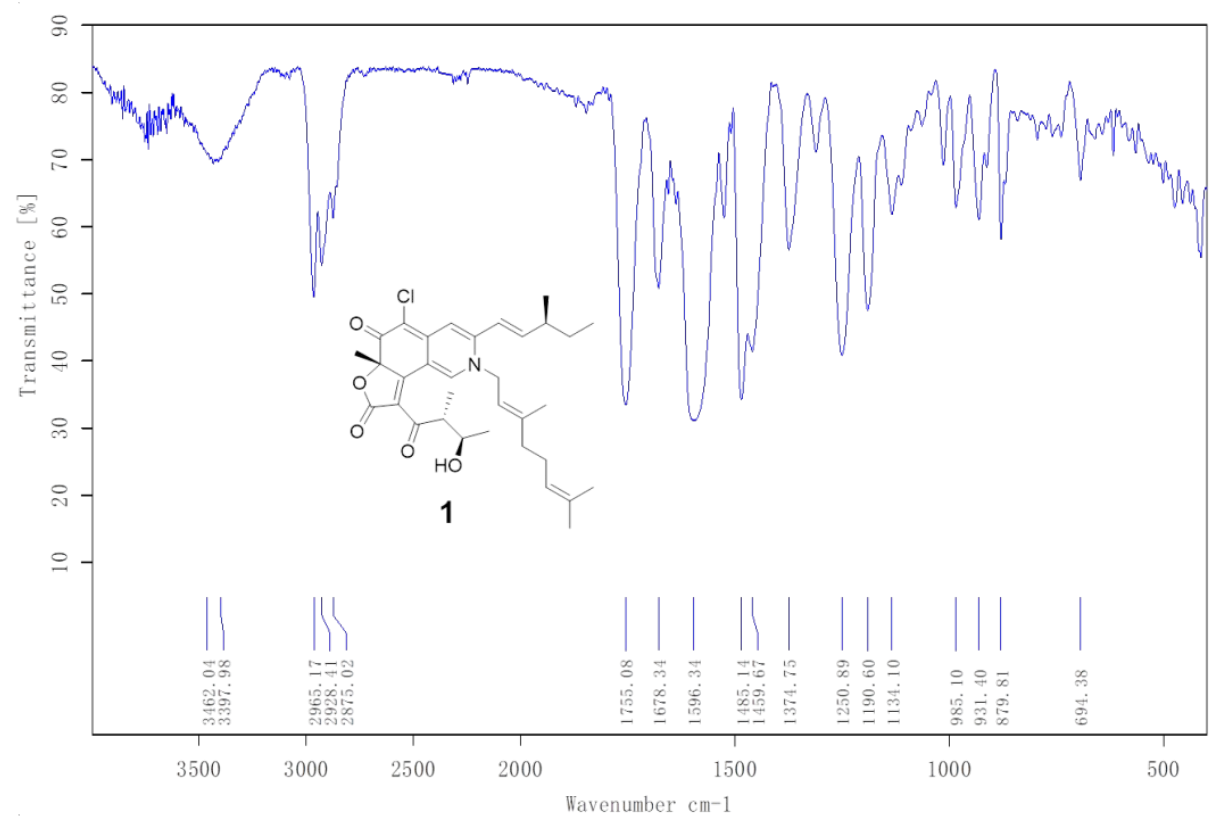

Figure S16. IR spectrum of compound 1 


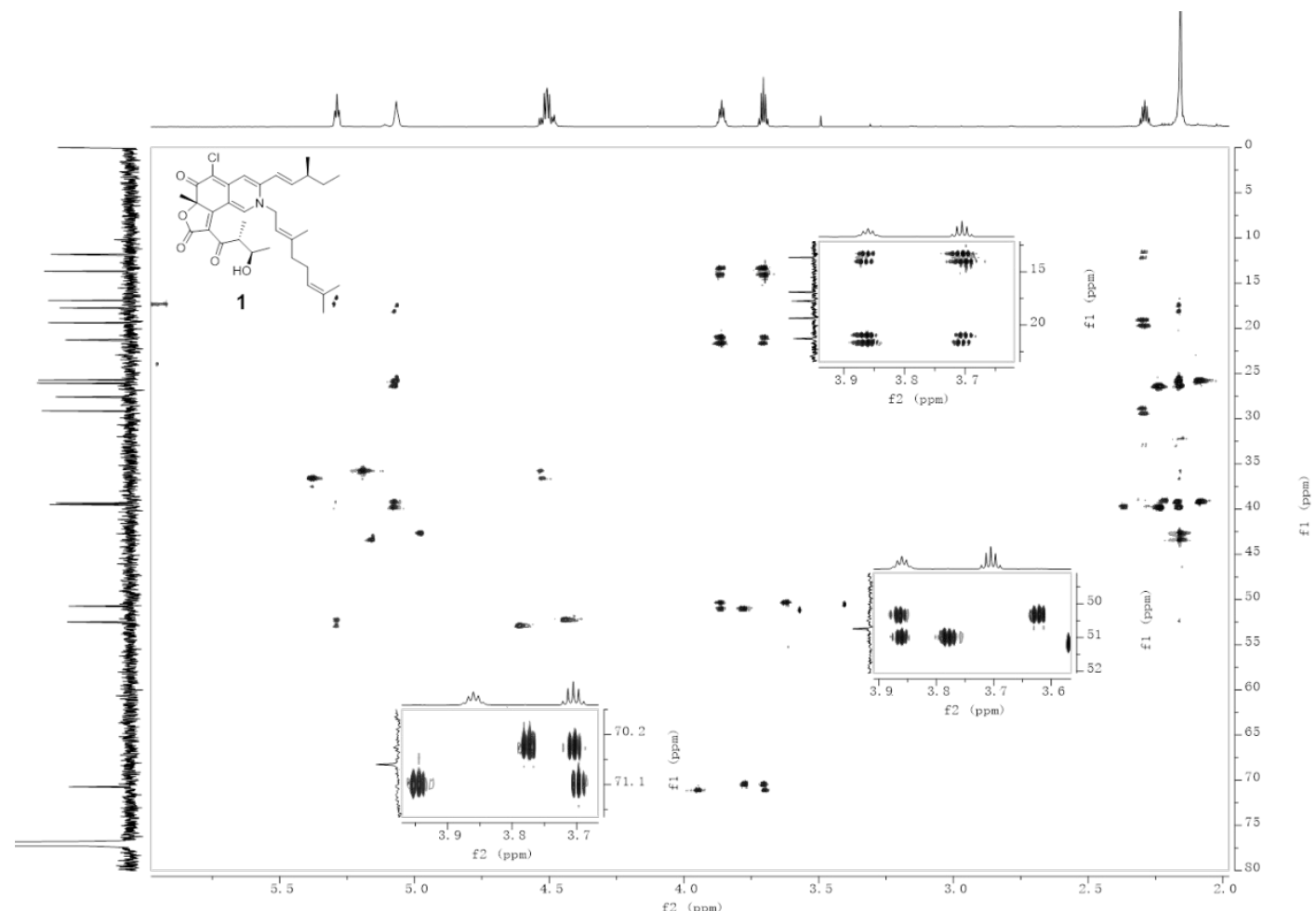

Figure S17. HSQC-HECADE spectra of compound 1

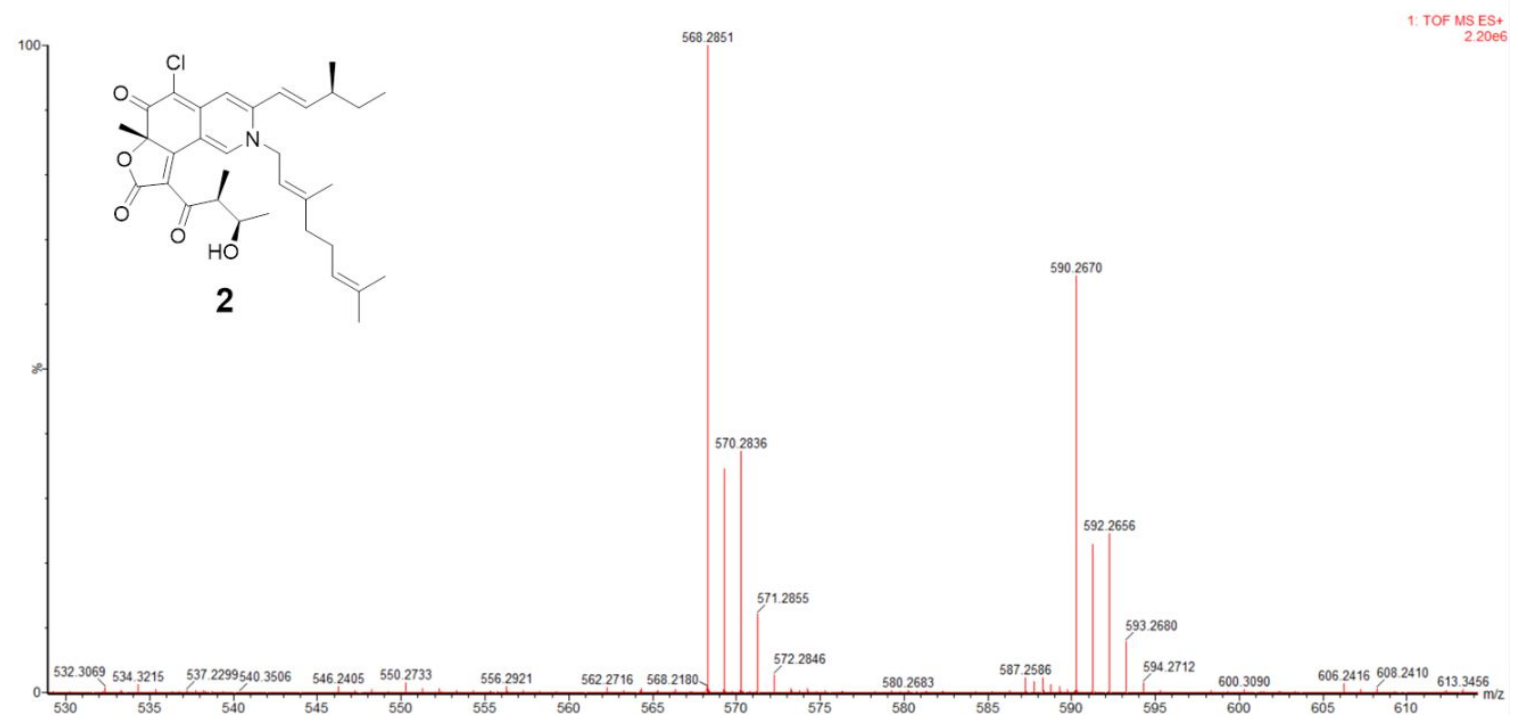

Figure S18. HRESIMS spectrum of compound 2 


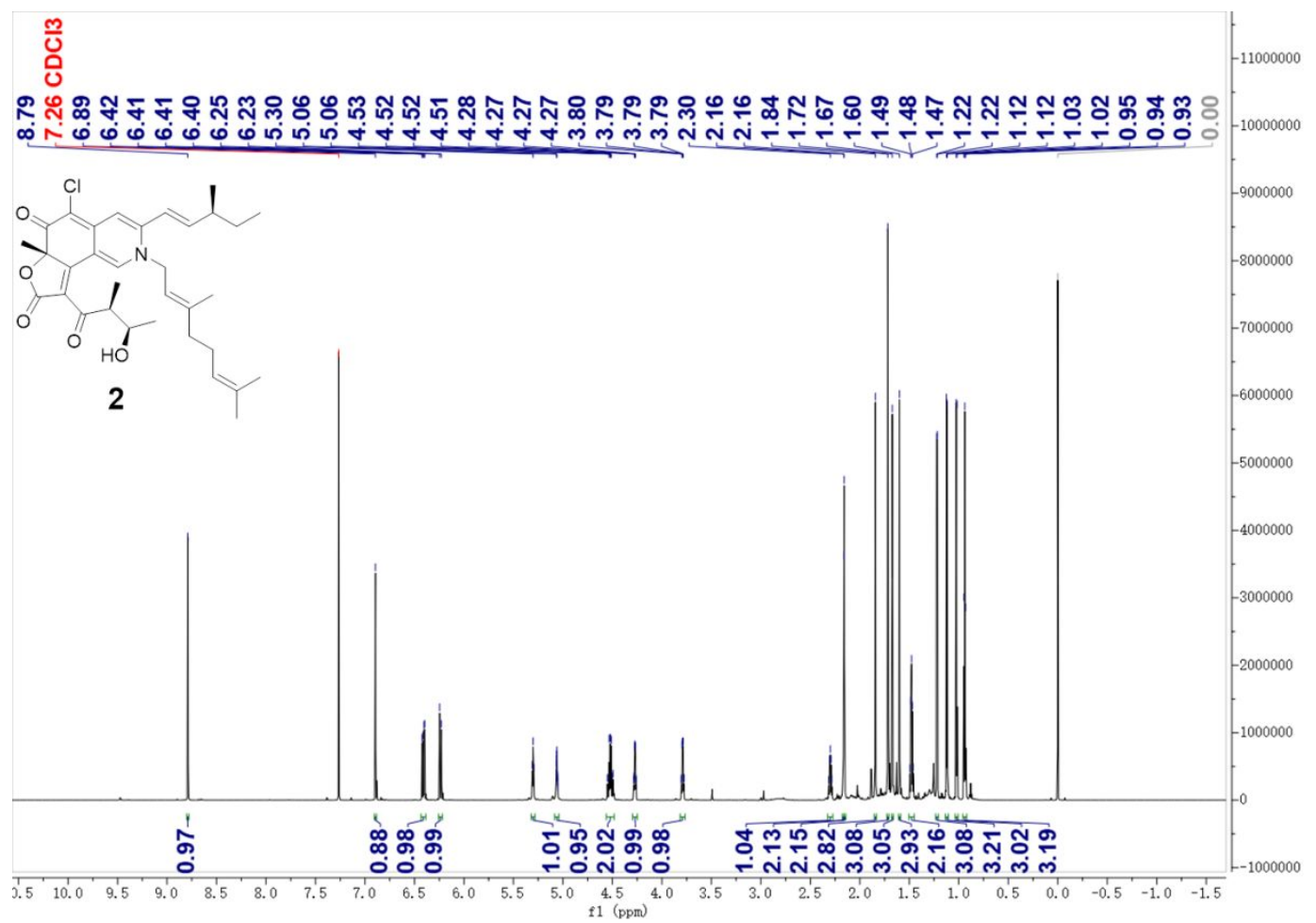

Figure S19. ${ }^{1} \mathrm{H}$ NMR spectrum of compound 2

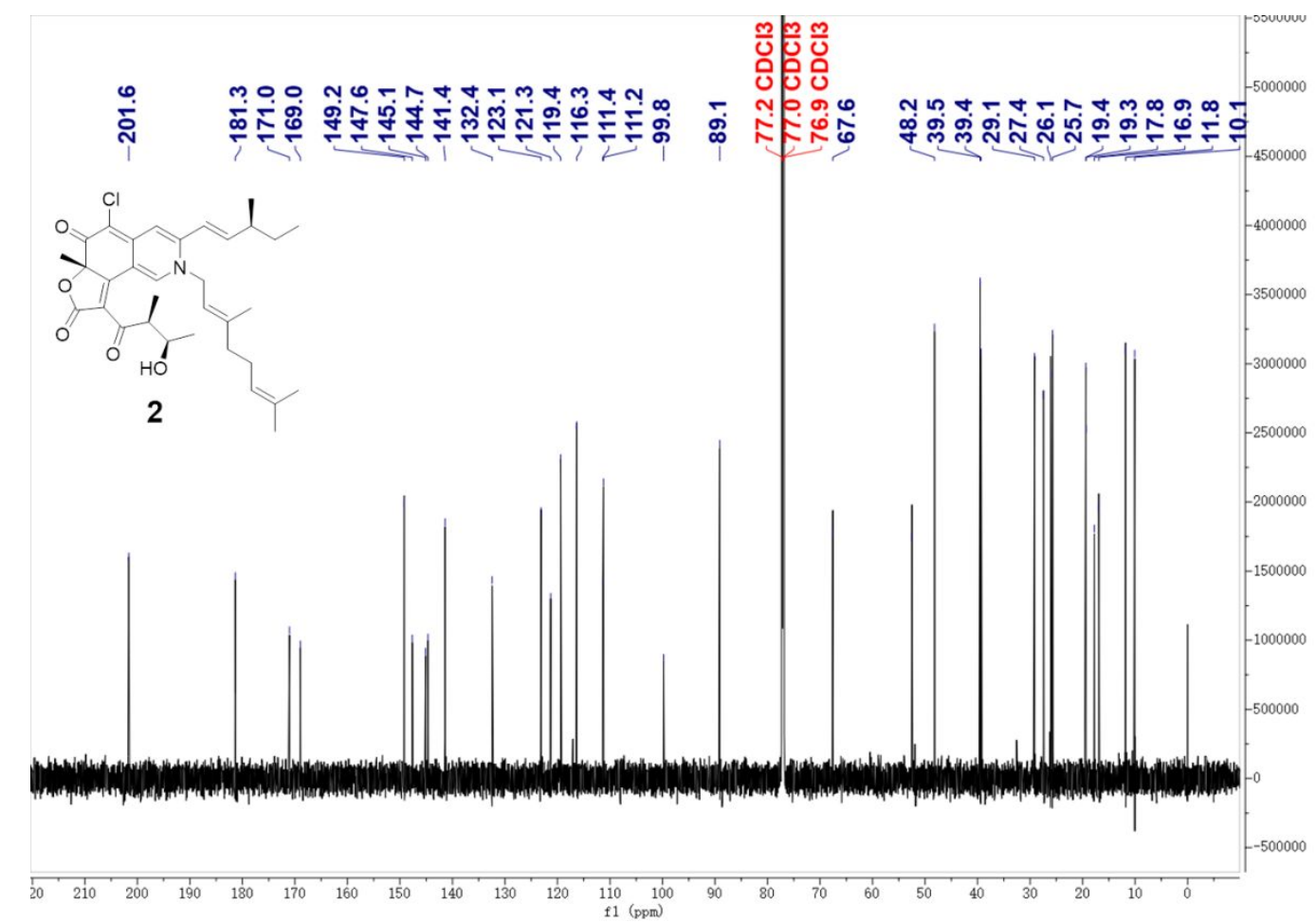

Figure S20. ${ }^{13} \mathrm{C}$ NMR spectrum of compound 2 


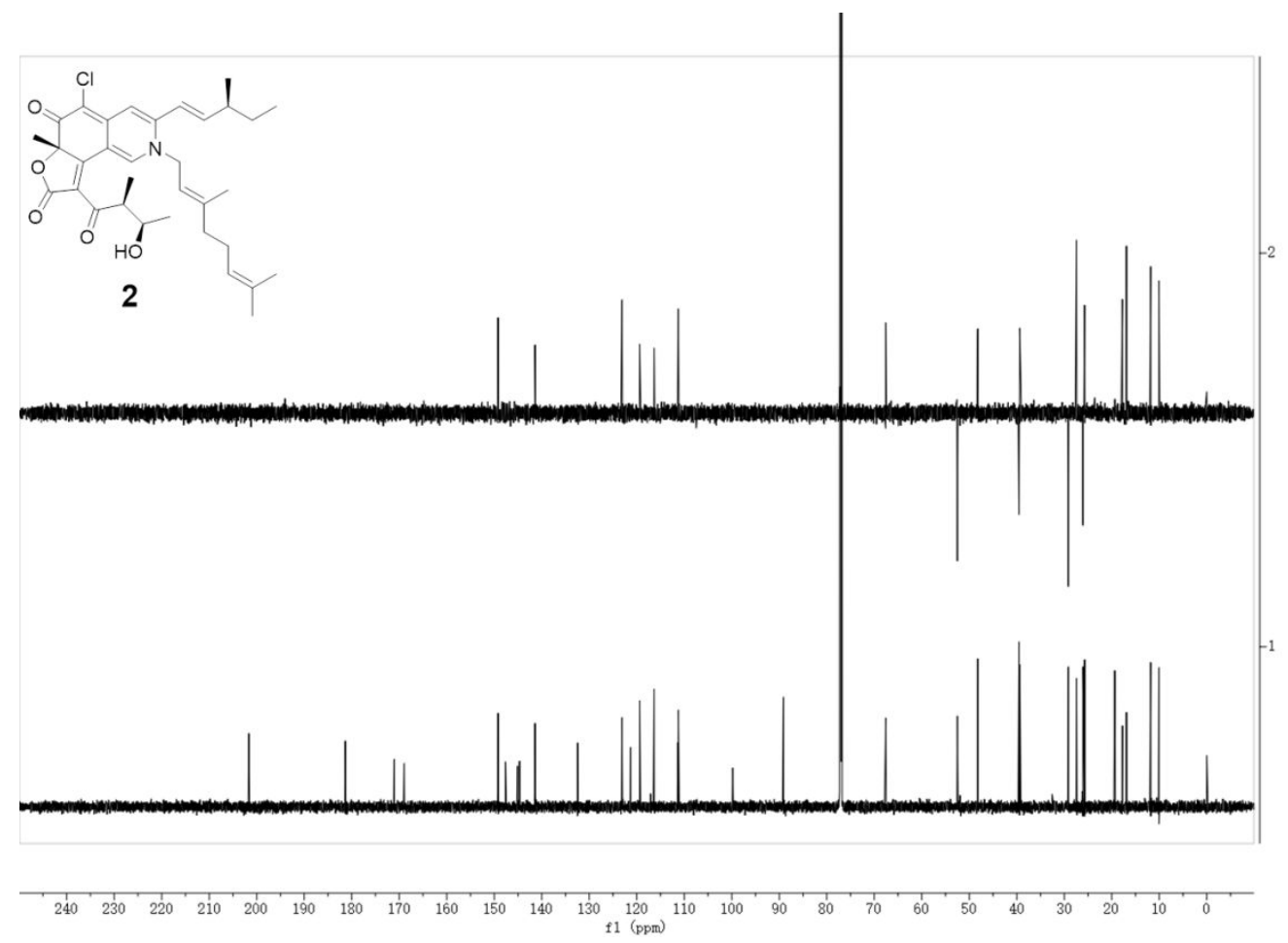

Figure S21. ${ }^{13} \mathrm{C} / \mathrm{DEPT}$ spectrum of compound 2

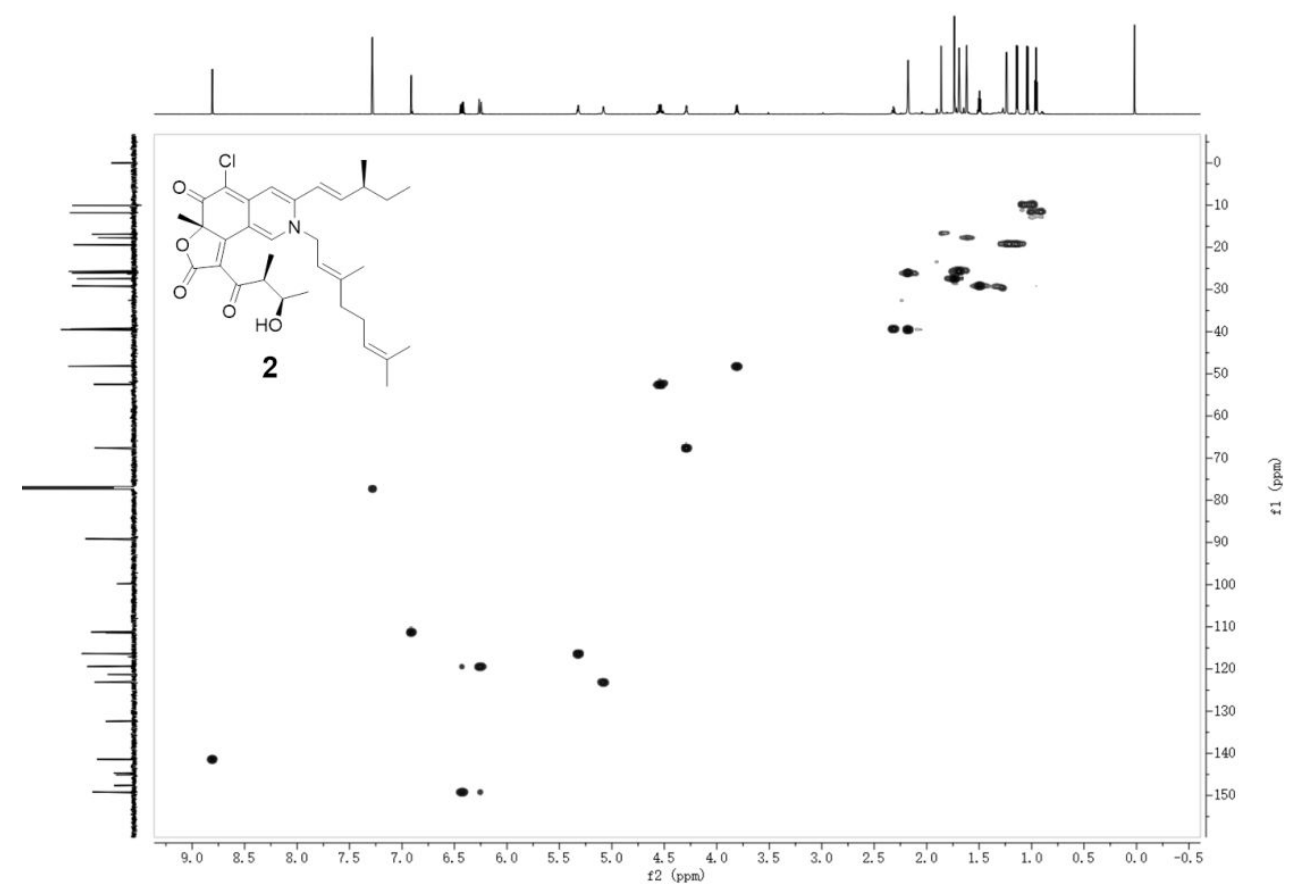

Figure S22. HSQC spectrum of compound 2 


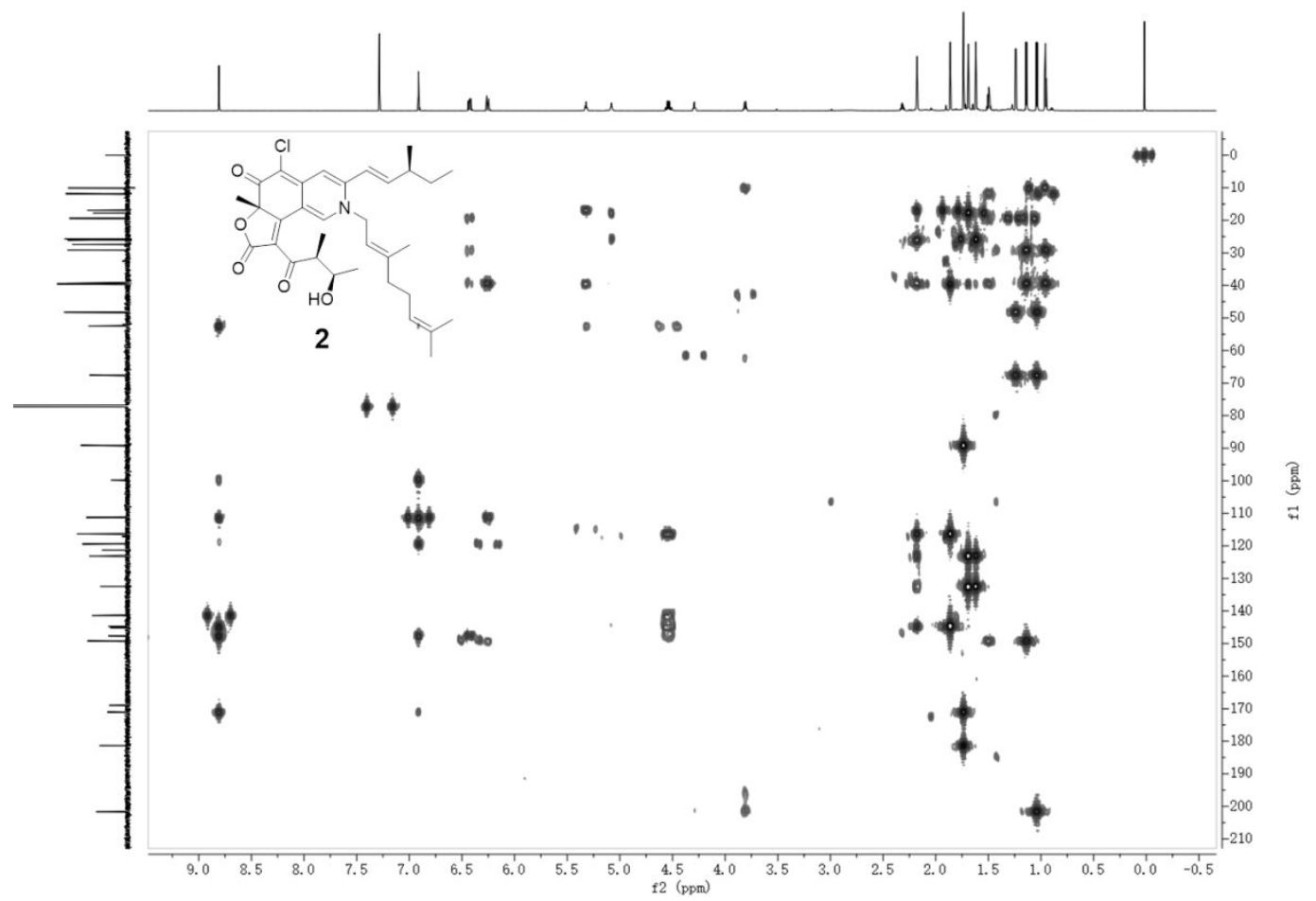

Figure S23. HMBC spectrum of compound 2

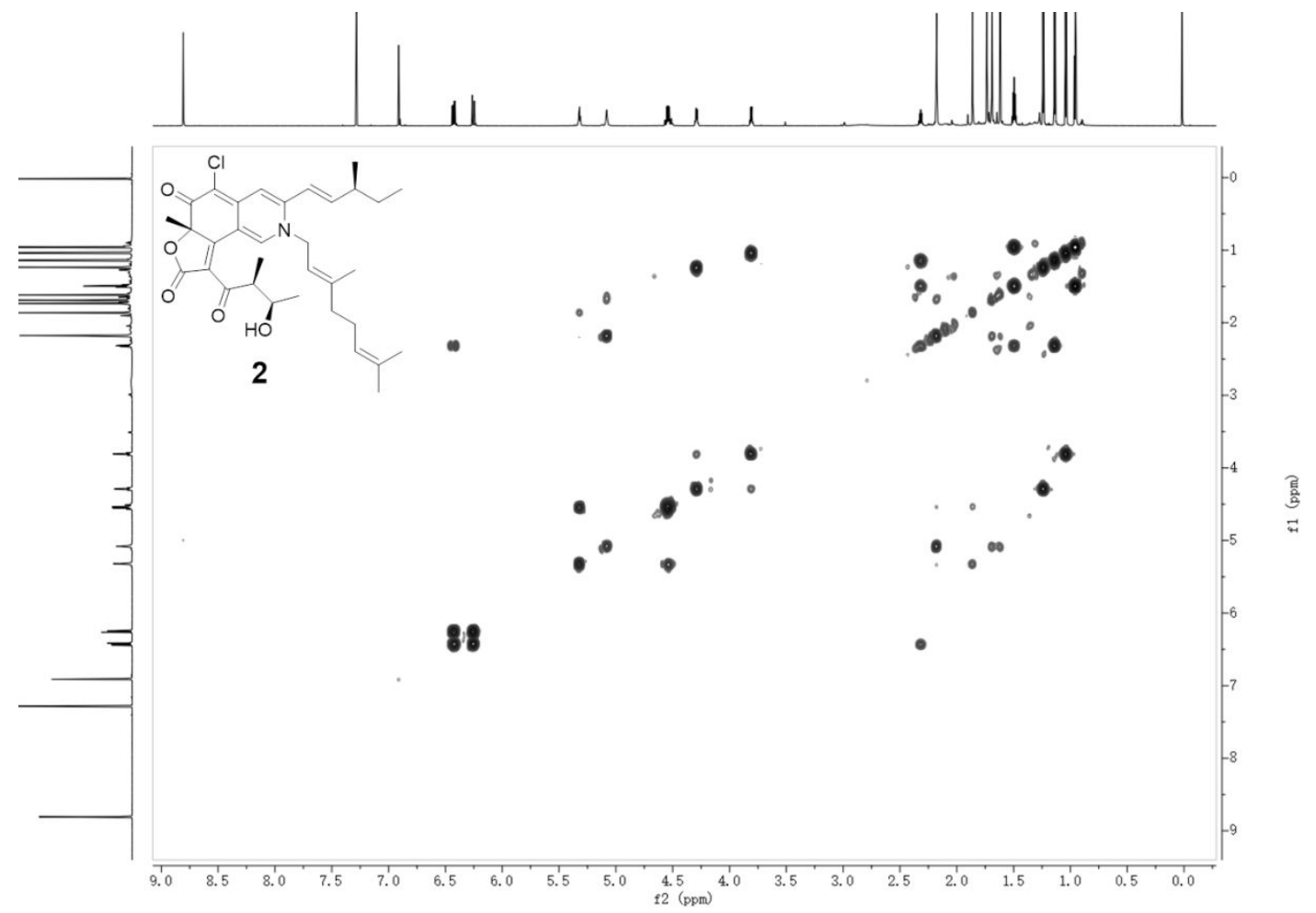

Figure S24. COSY spectrum of compound 2 


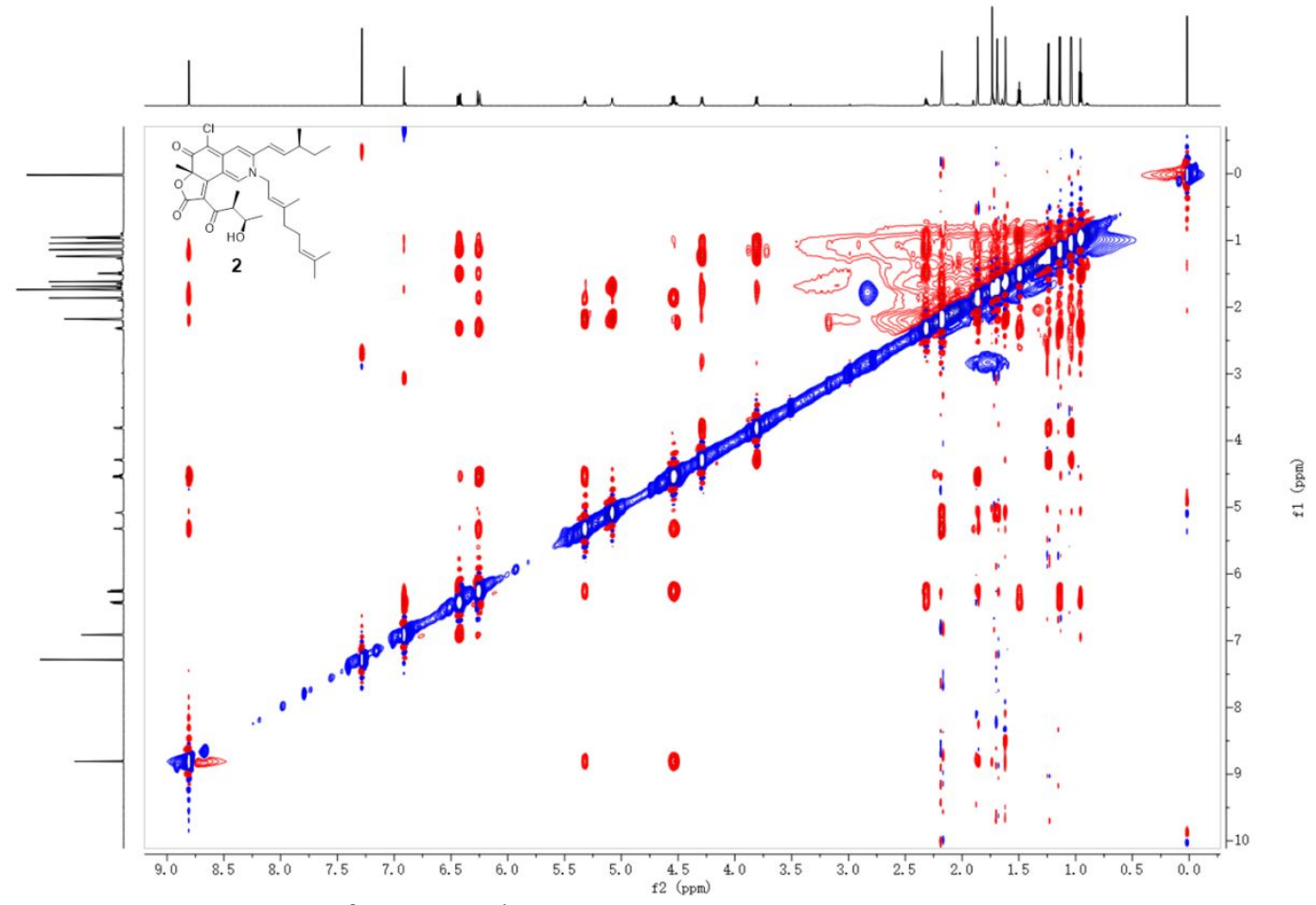

Figure S25. NOESY spectrum of compound 2

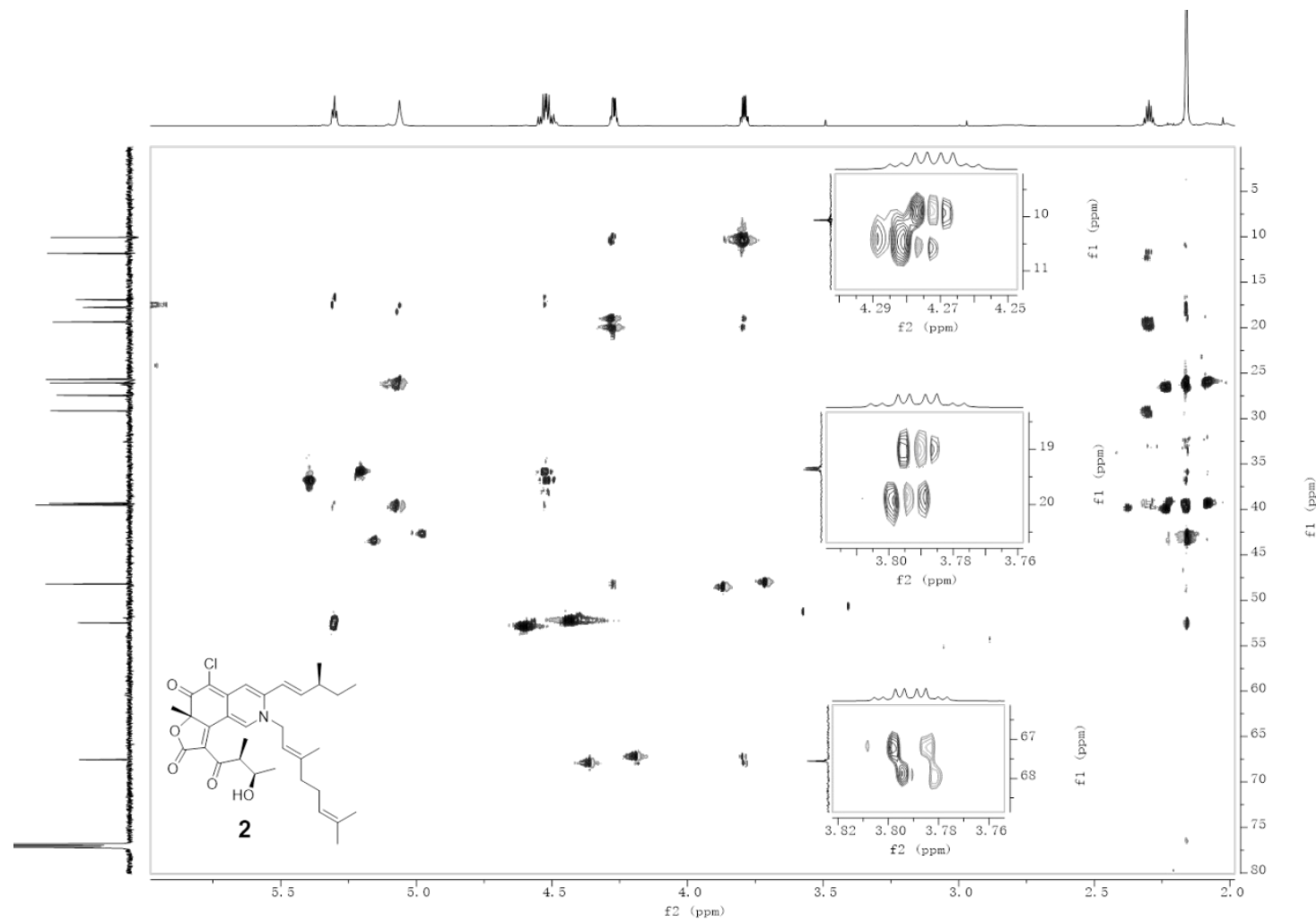

Figure S26. HSQC-HECADE spectra of compound 2 


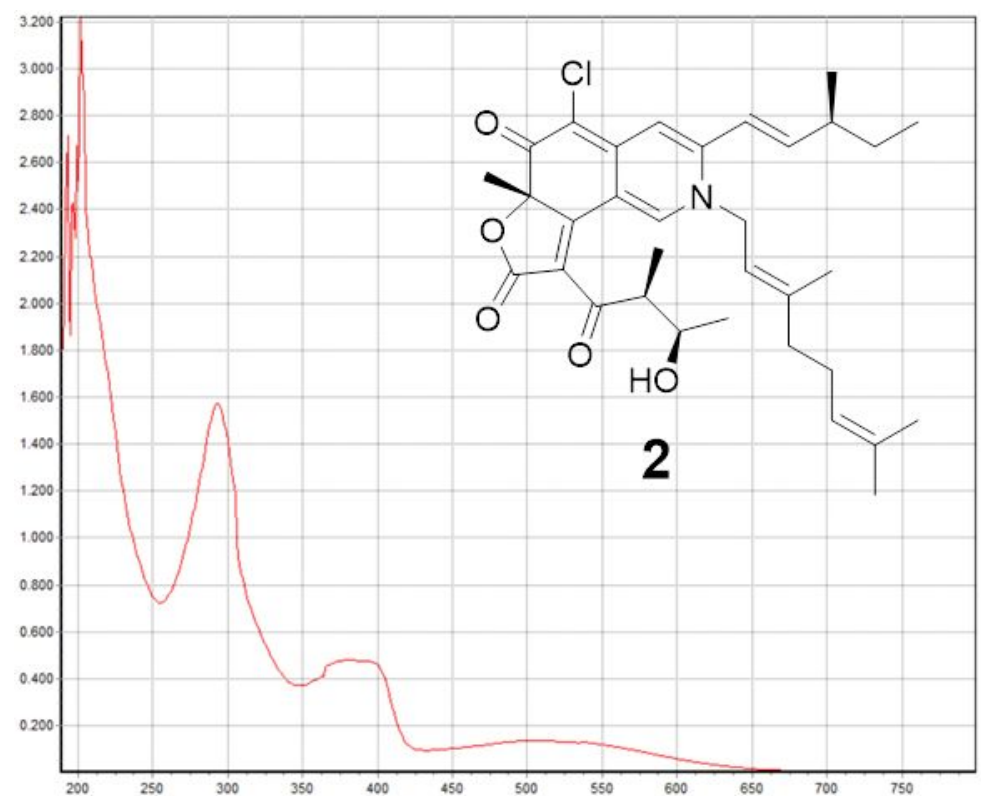

Figure S27. UV spectrum of compound 2

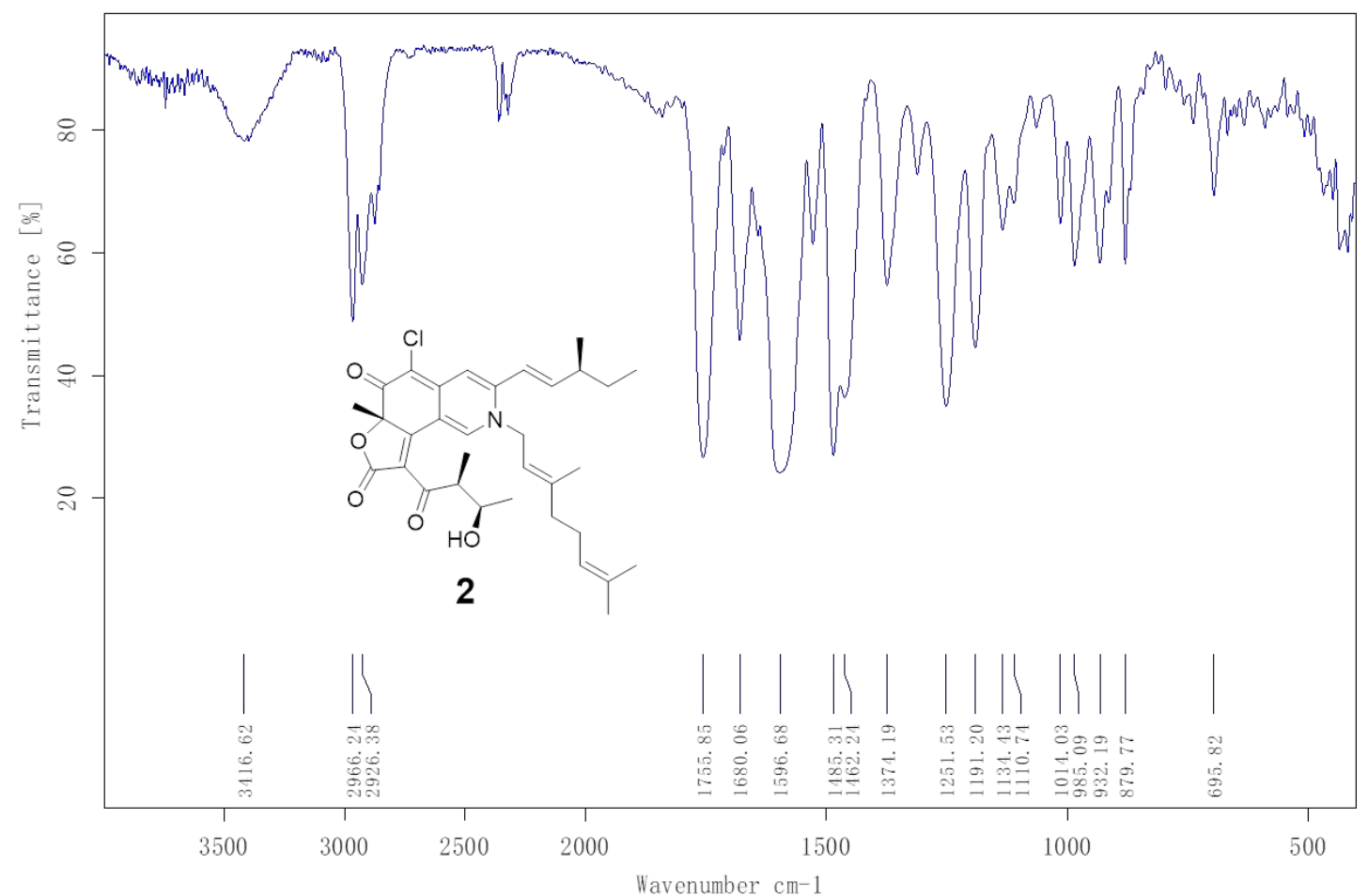

Figure S28. IR spectrum of compound 2 


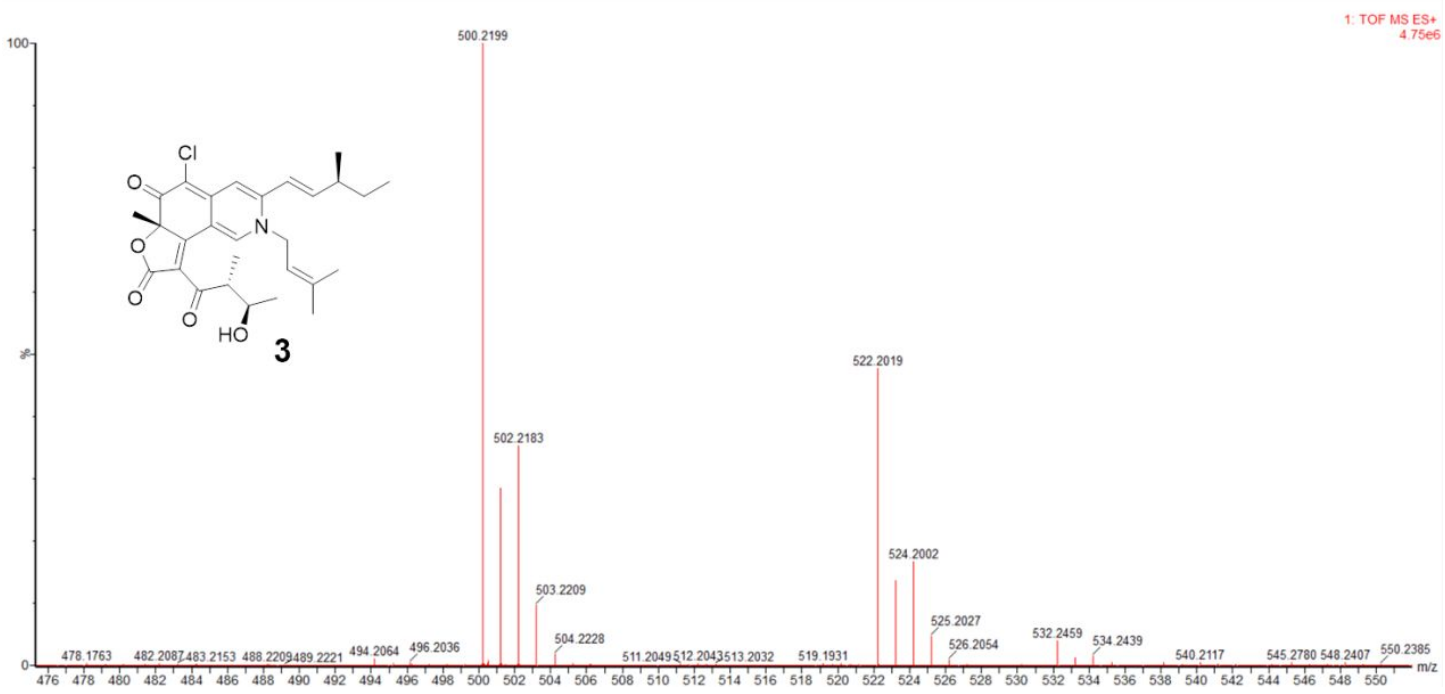

Figure S29. HRESIMS spectrum of compound $\mathbf{3}$

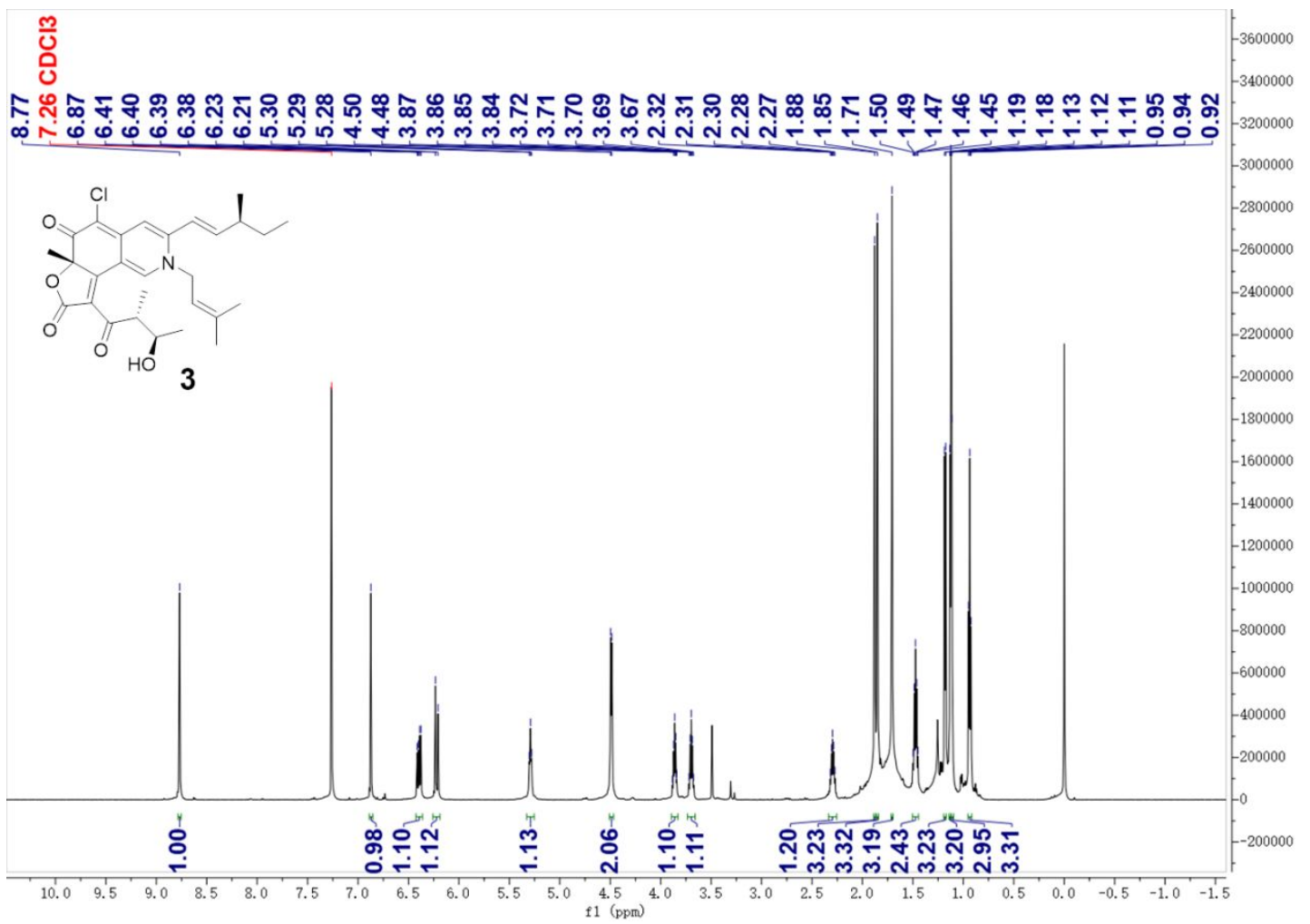

Figure S30. ${ }^{1} \mathrm{H}$ NMR spectrum of compound 3 


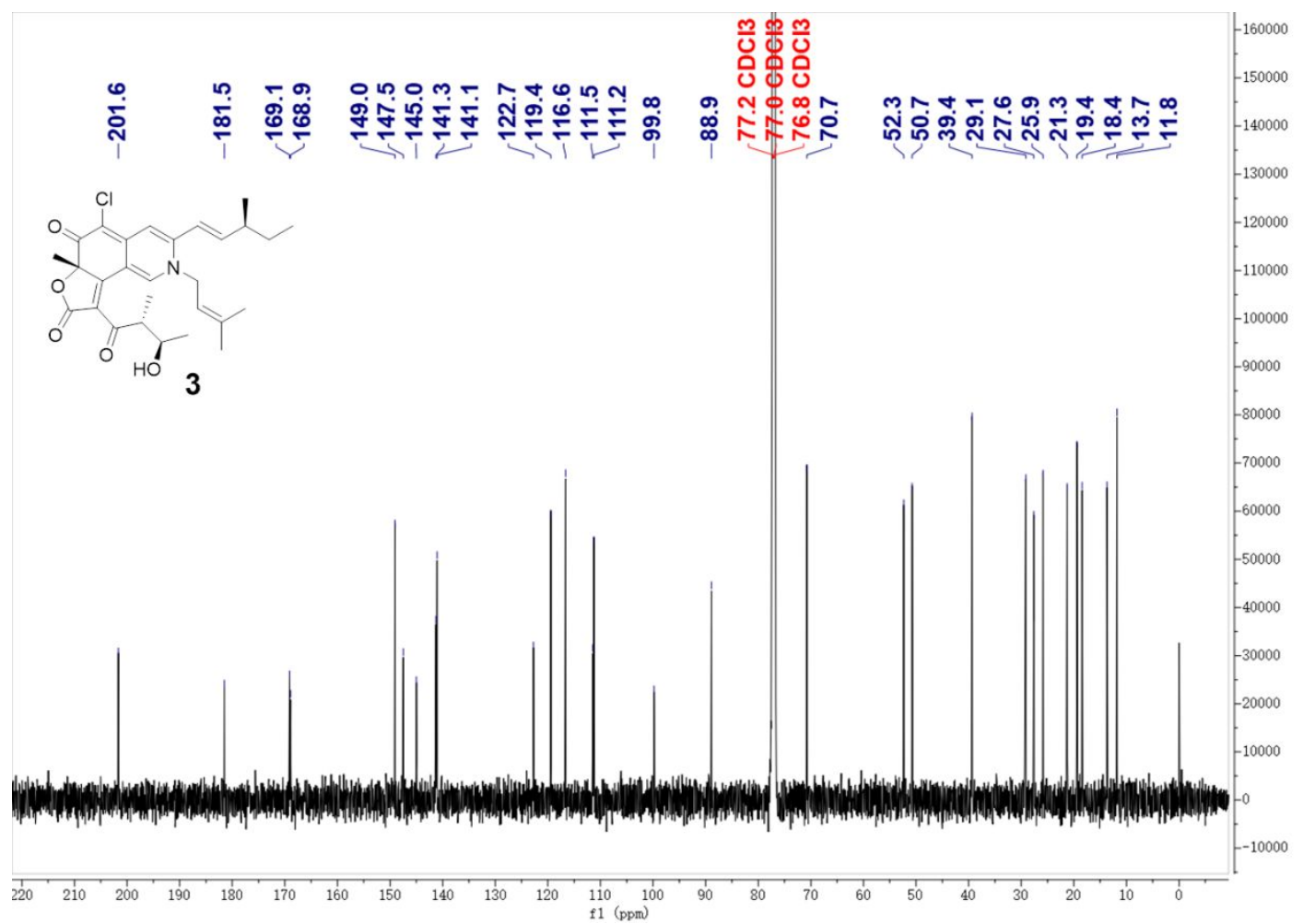

Figure S31. ${ }^{13} \mathrm{C}$ NMR spectrum of compound 3
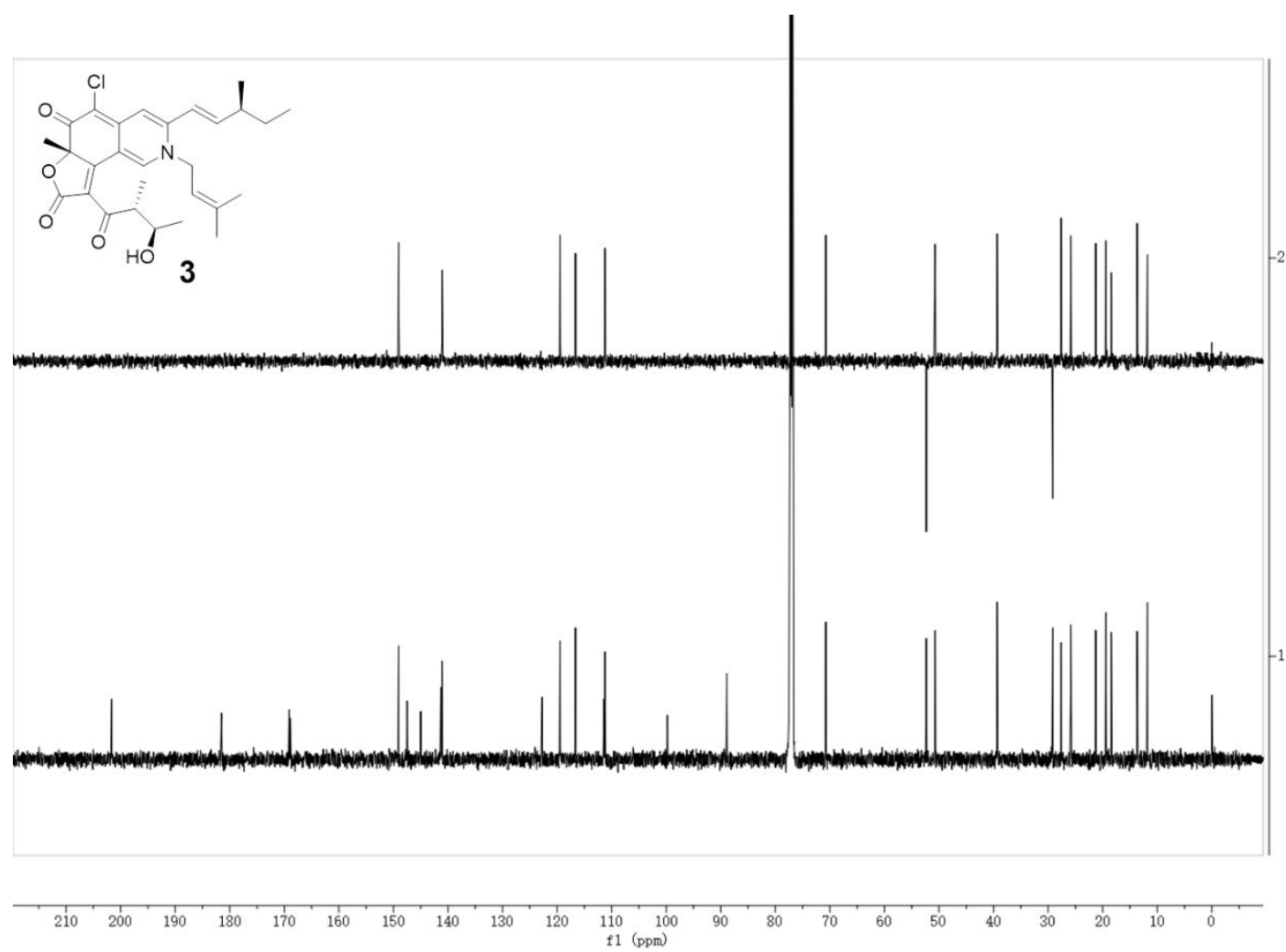

Figure S32. ${ }^{13} \mathrm{C} / \mathrm{DEPT}$ spectrum of compound 3 


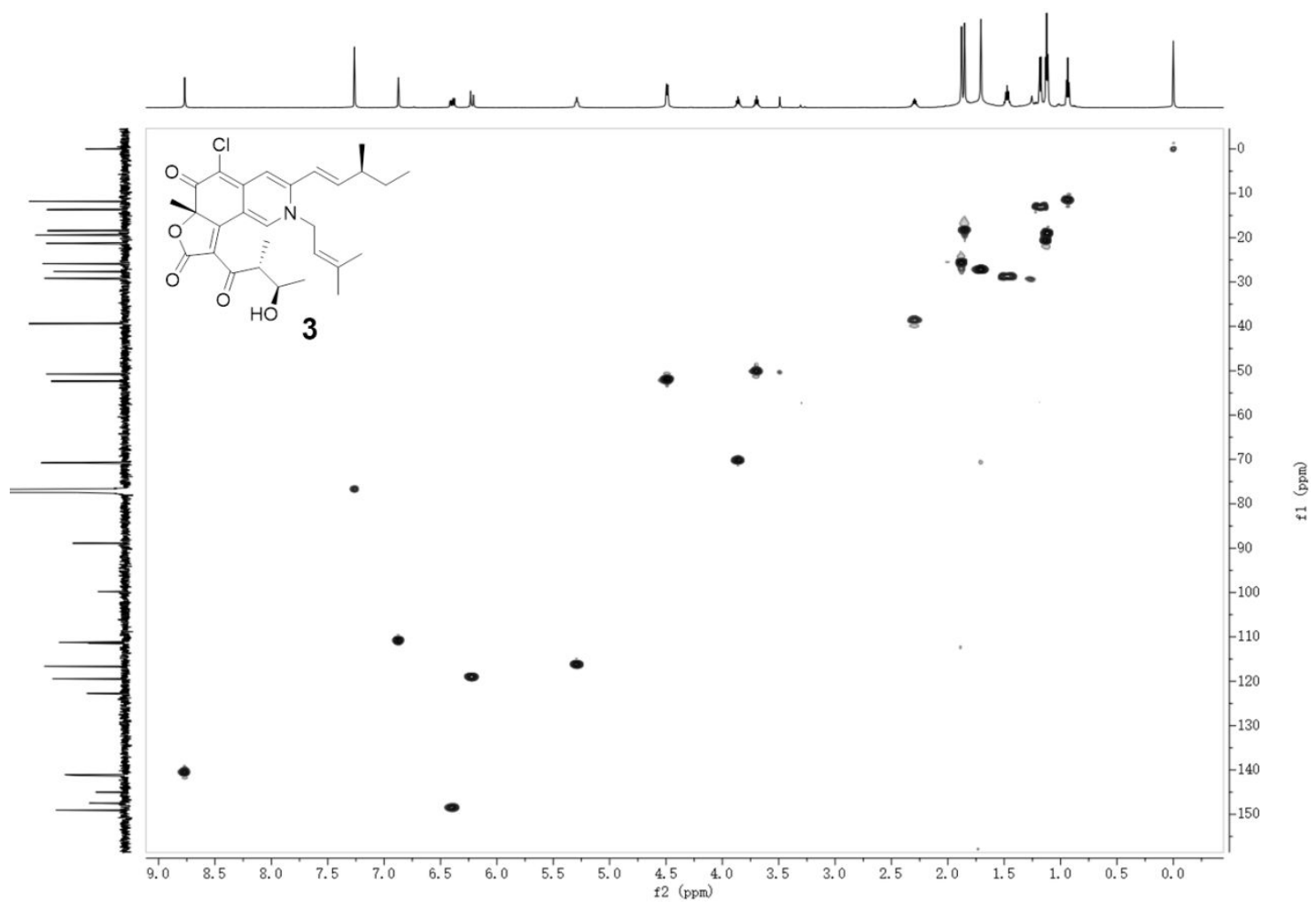

Figure S33. HSQC spectrum of compound 3

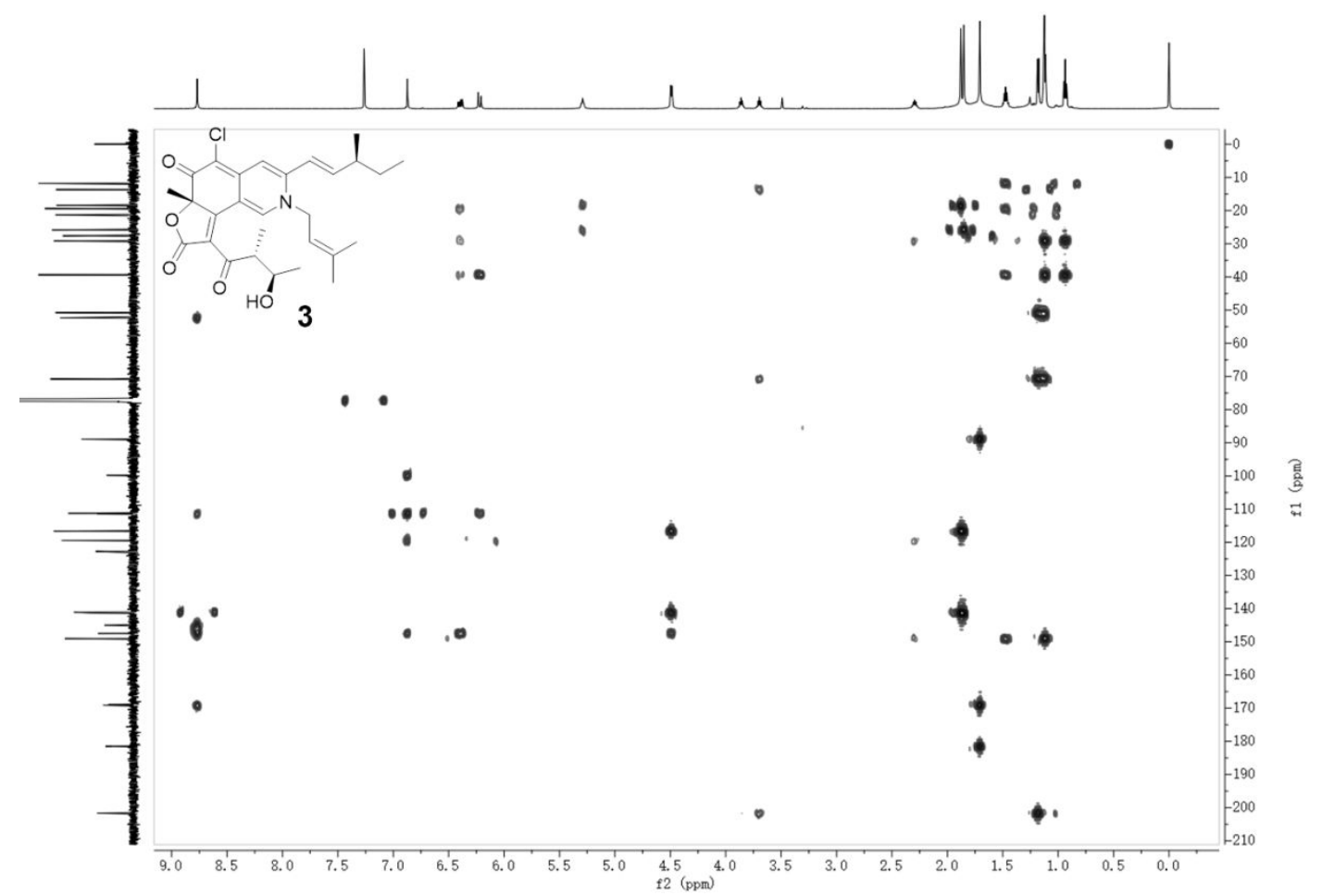

Figure S34. HMBC spectrum of compound 3 


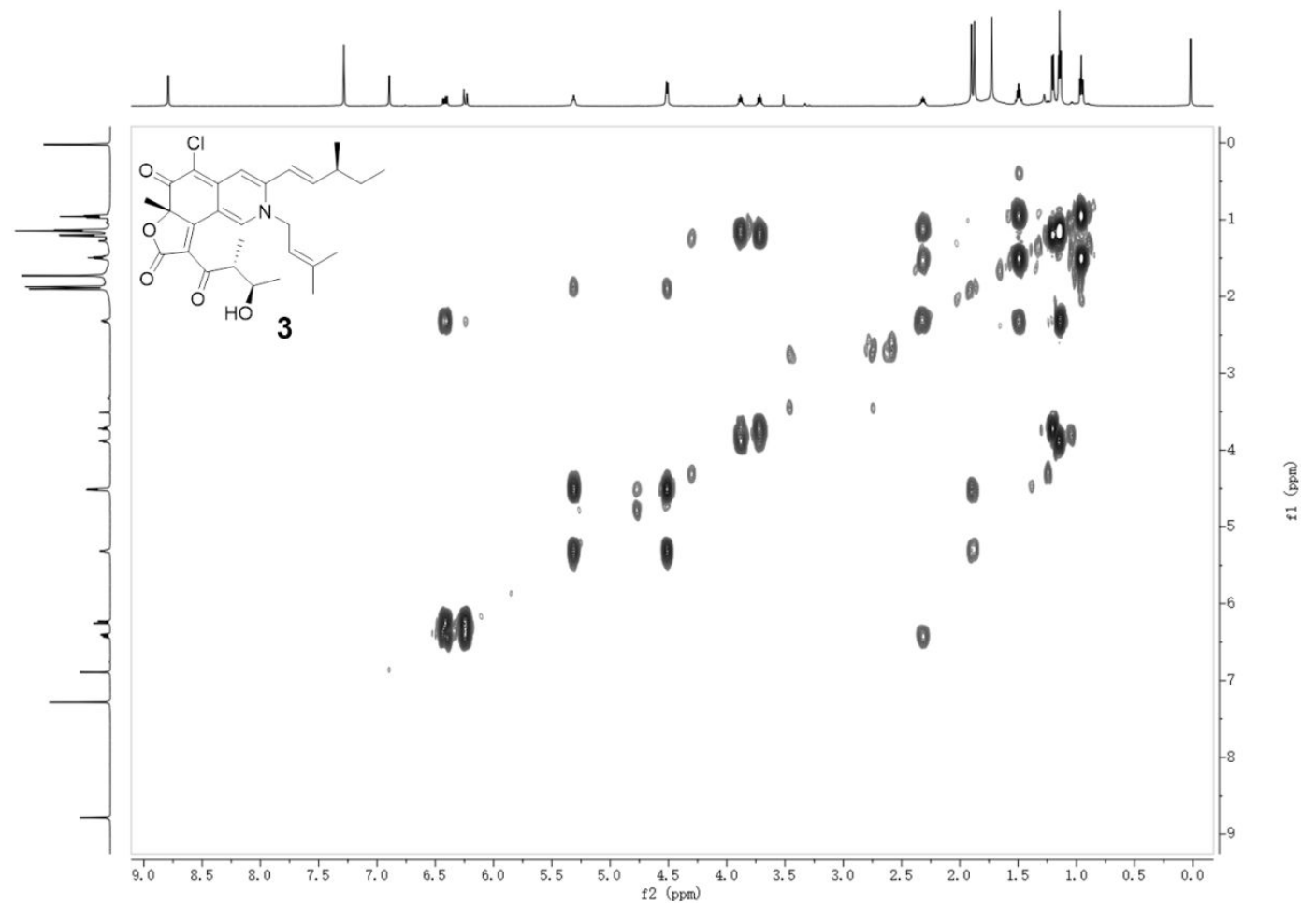

Figure S35. COSY spectrum of compound 3

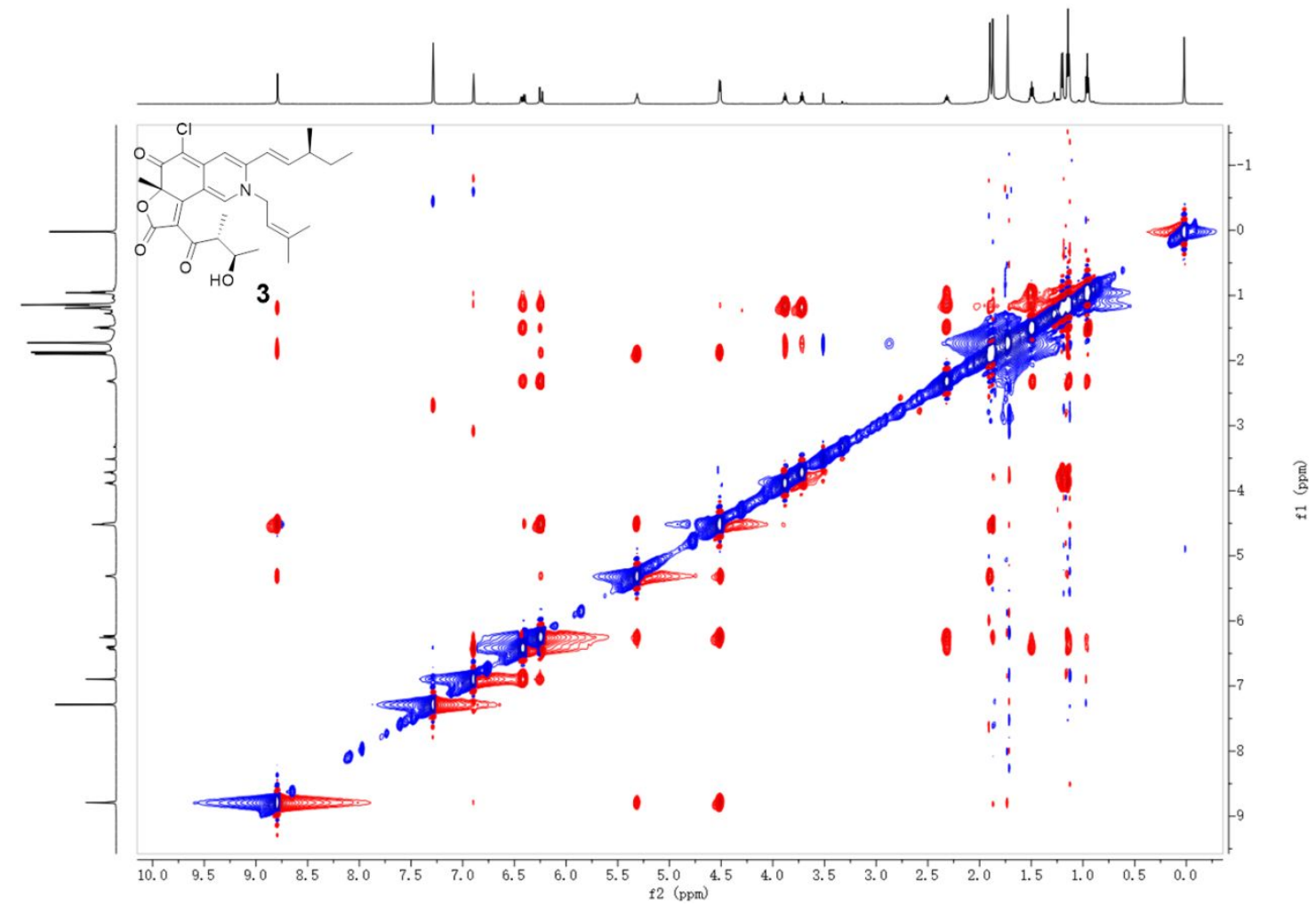

Figure S36. NOESY spectrum of compound $\mathbf{3}$ 


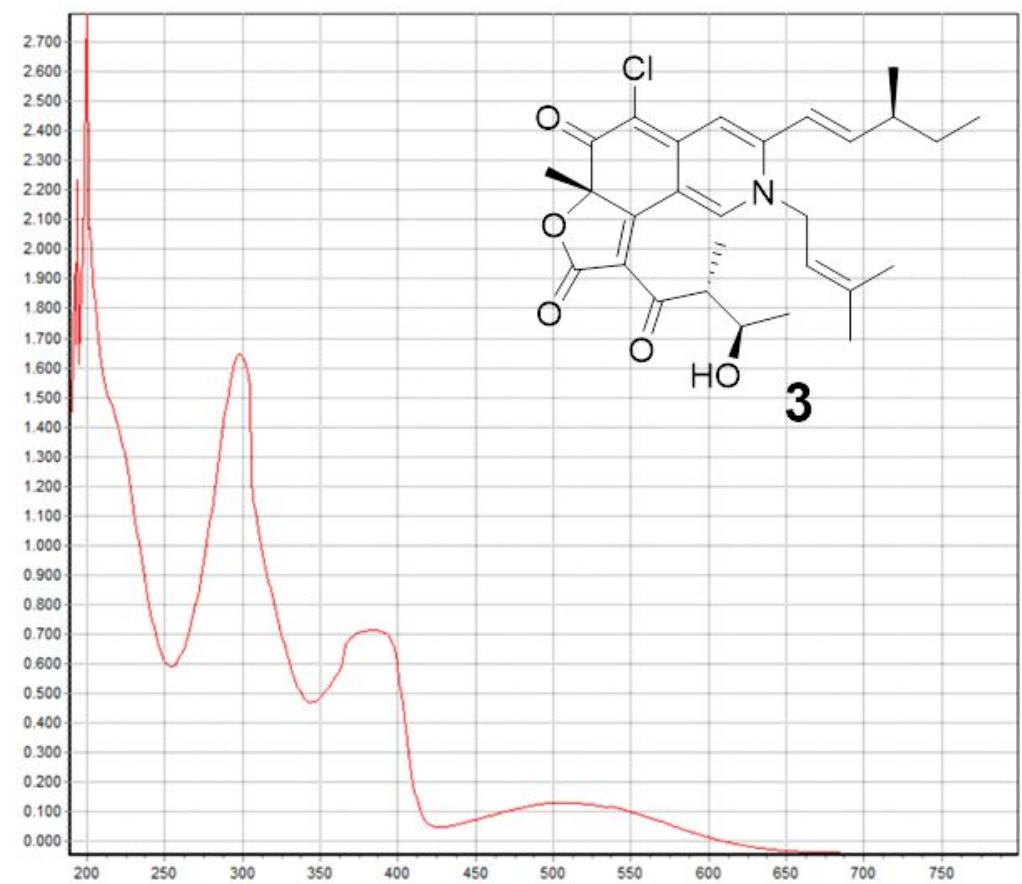

Figure S37. UV spectrum of compound 3

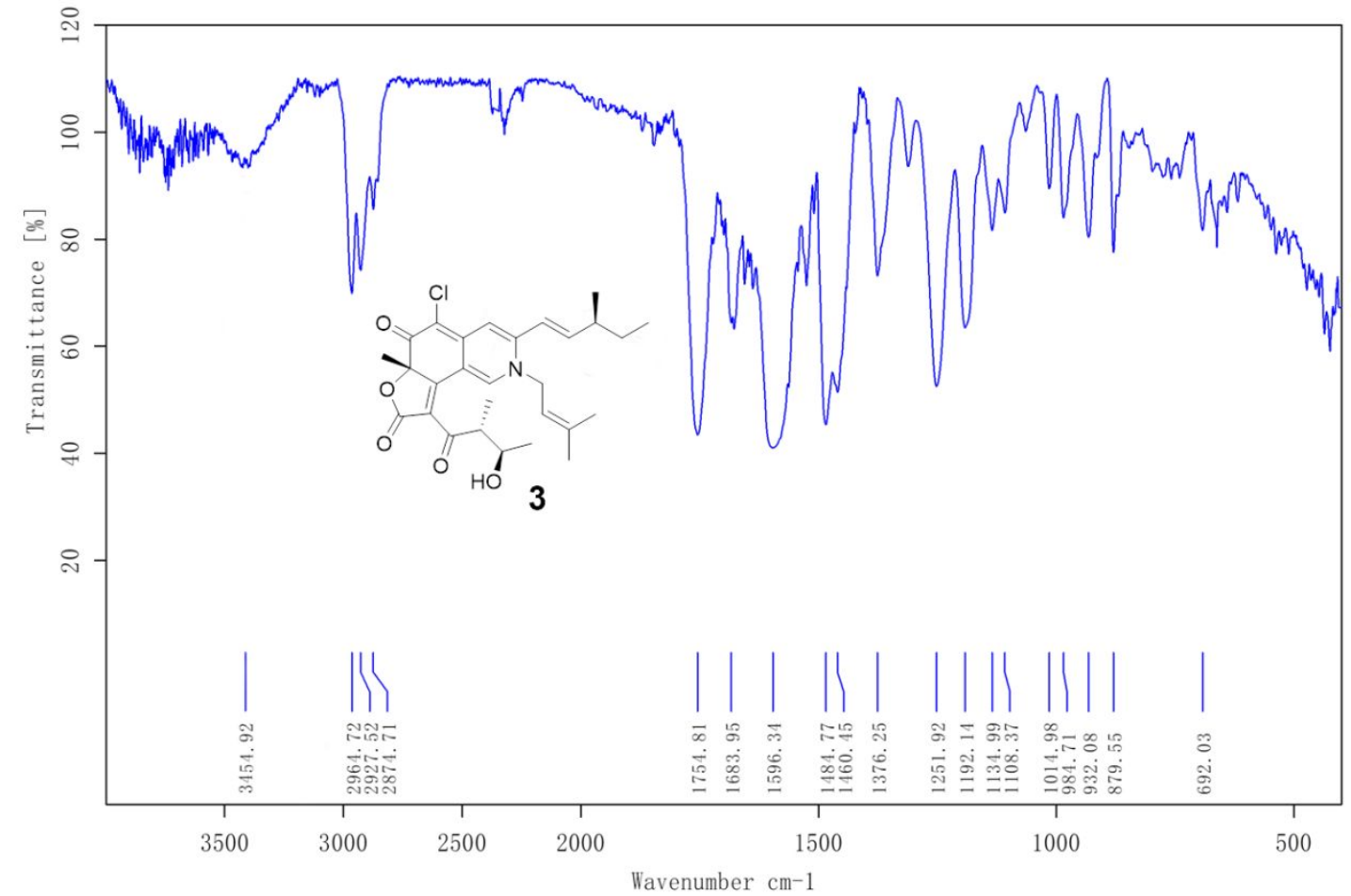

Figure S38. IR spectrum of compound 3 


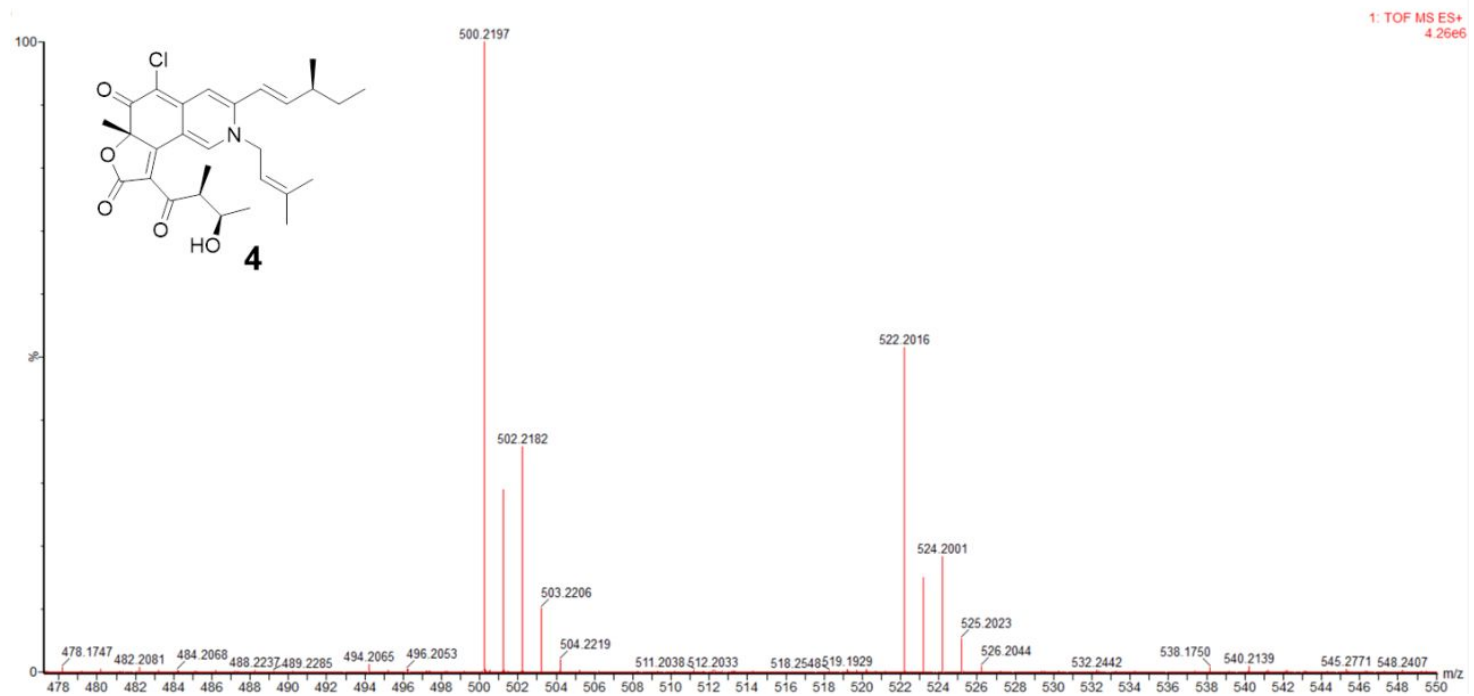

Figure S39. HRESIMS spectrum of compound 4

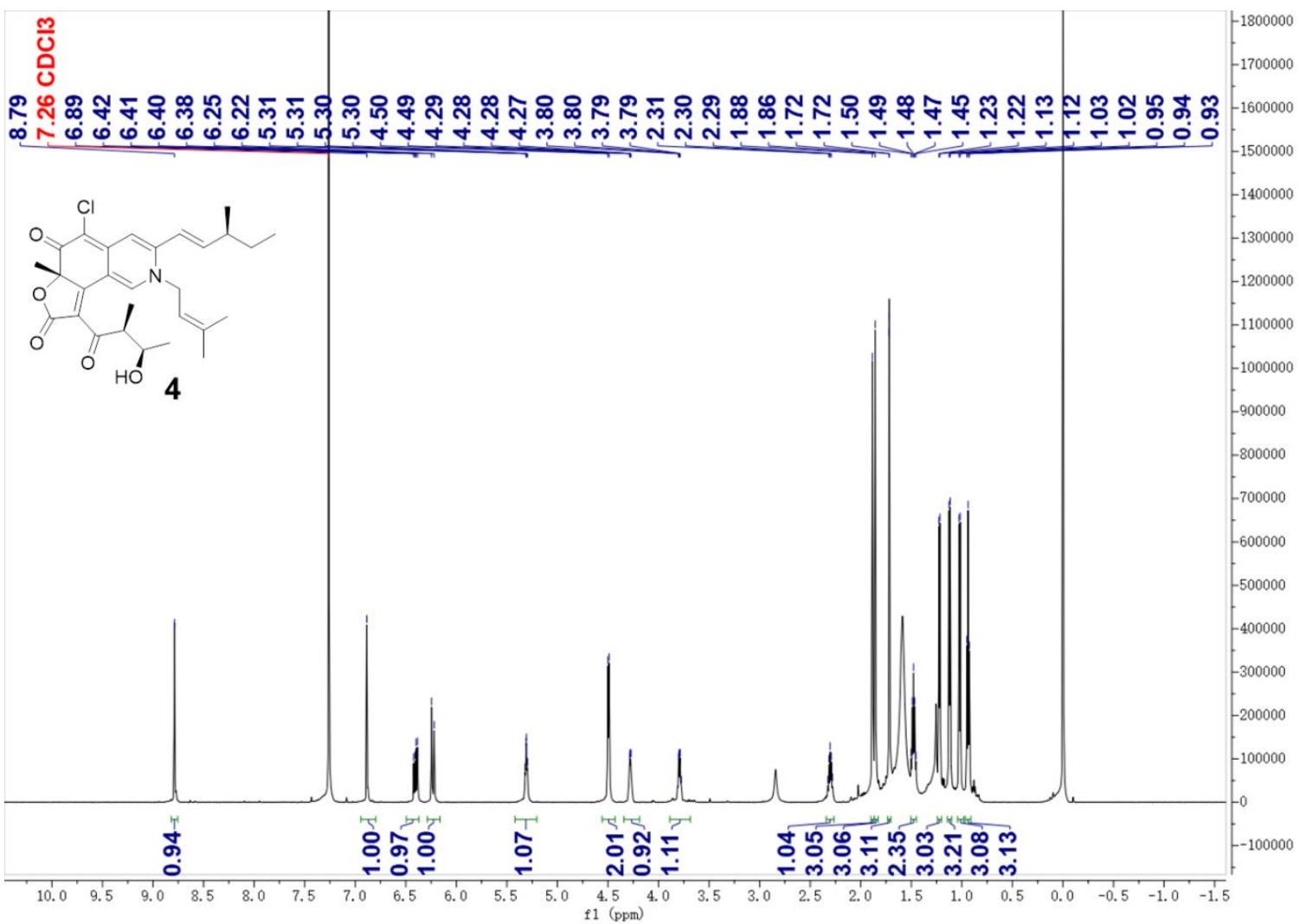

Figure S40. ${ }^{1} \mathrm{H}$ NMR spectrum of compound 4 


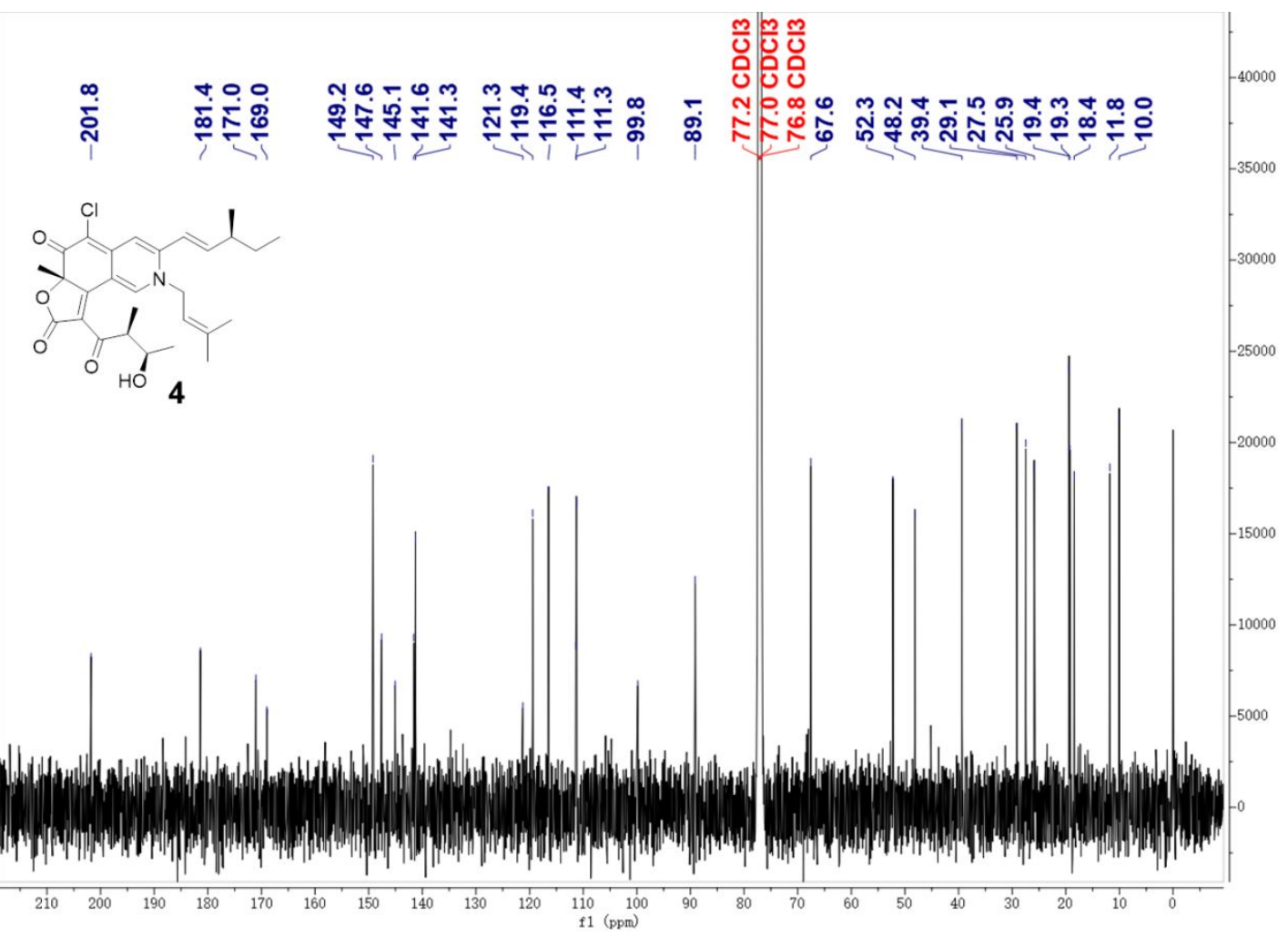

Figure S41. ${ }^{13} \mathrm{C}$ NMR spectrum of compound 4
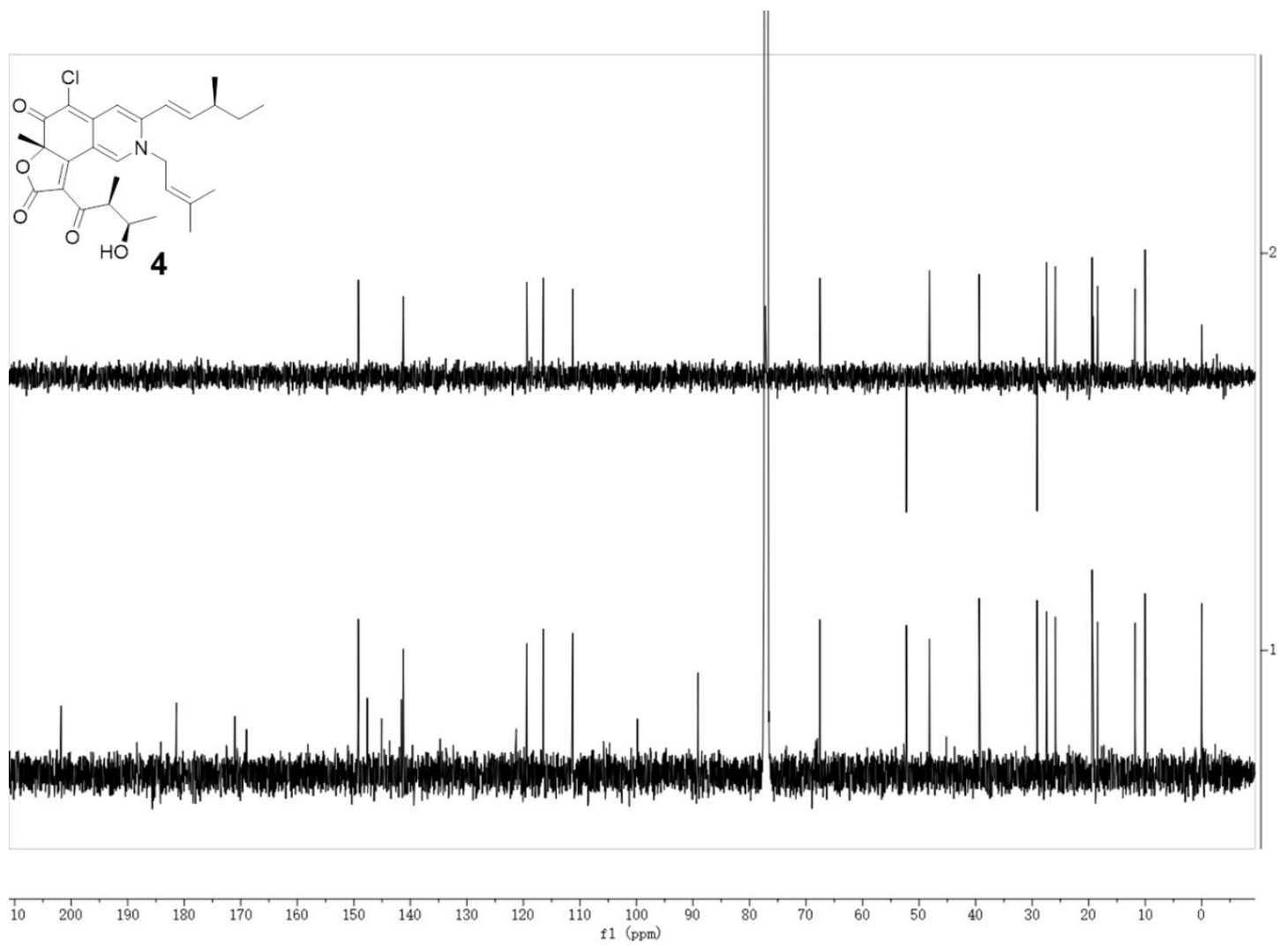

Figure S42. ${ }^{13} \mathrm{C} / \mathrm{DEPT}$ spectrum of compound 4 


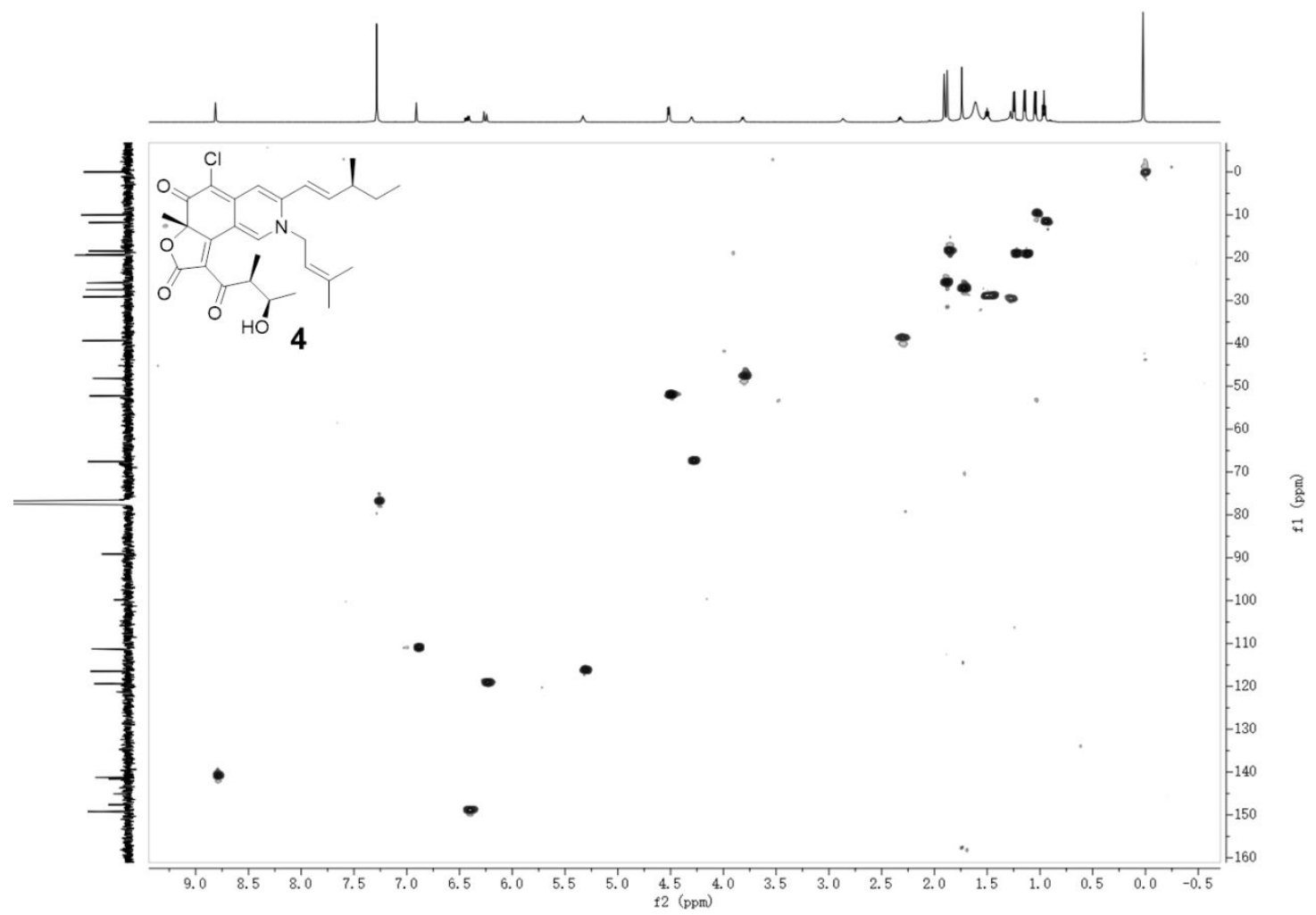

Figure S43. HSQC spectrum of compound 4

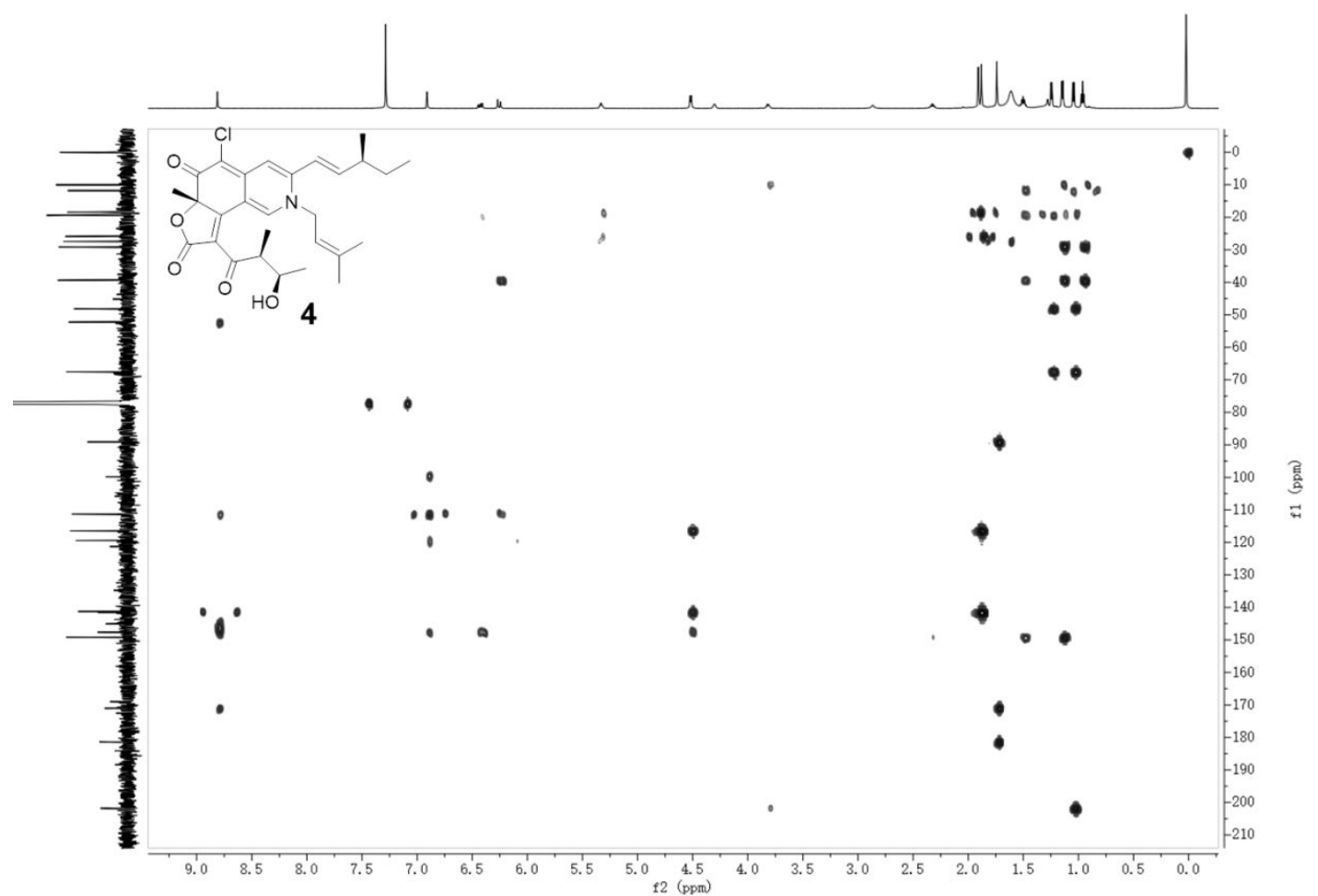

Figure S44. HMBC spectrum of compound 4 


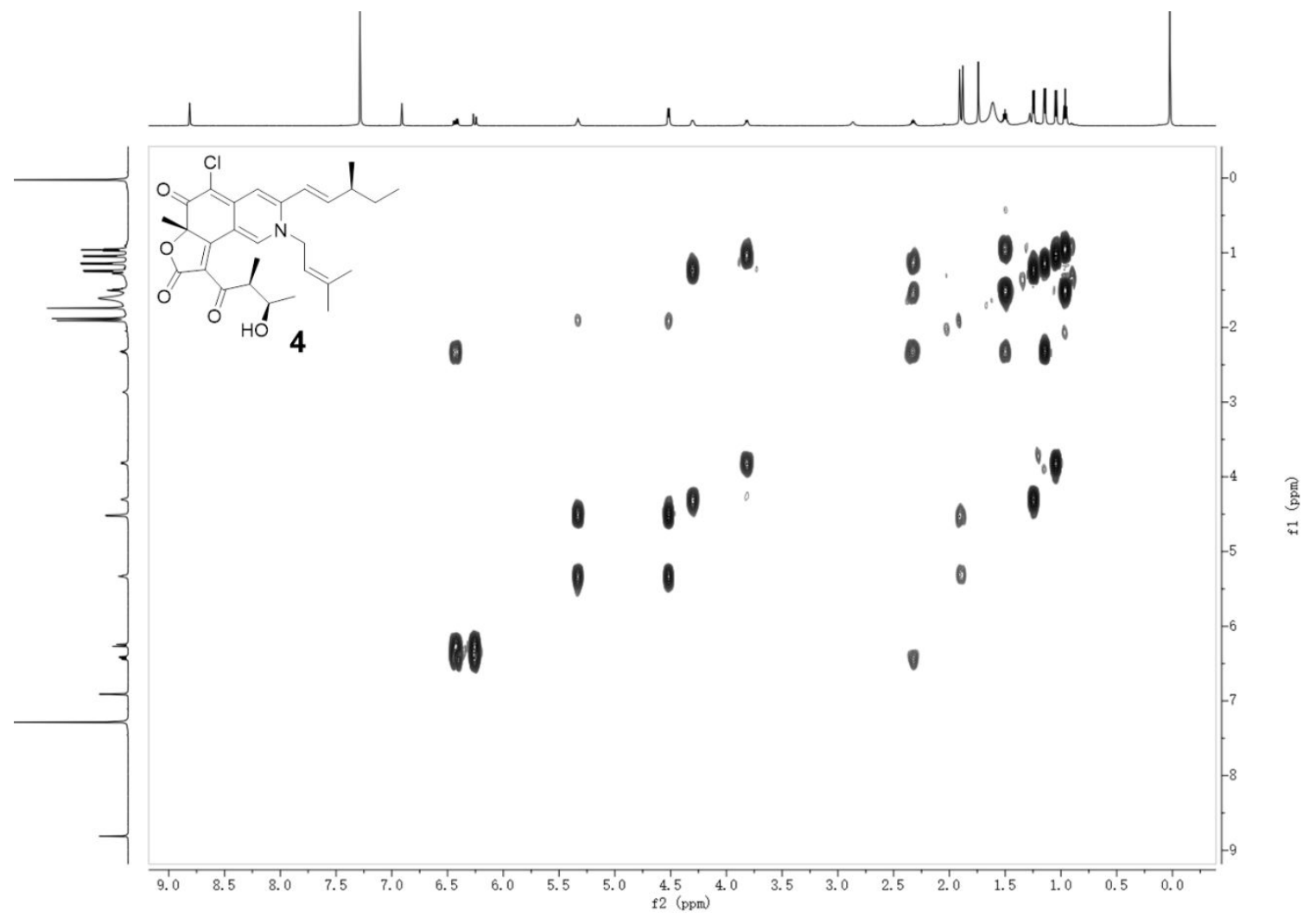

Figure S45. COSY spectrum of compound 4

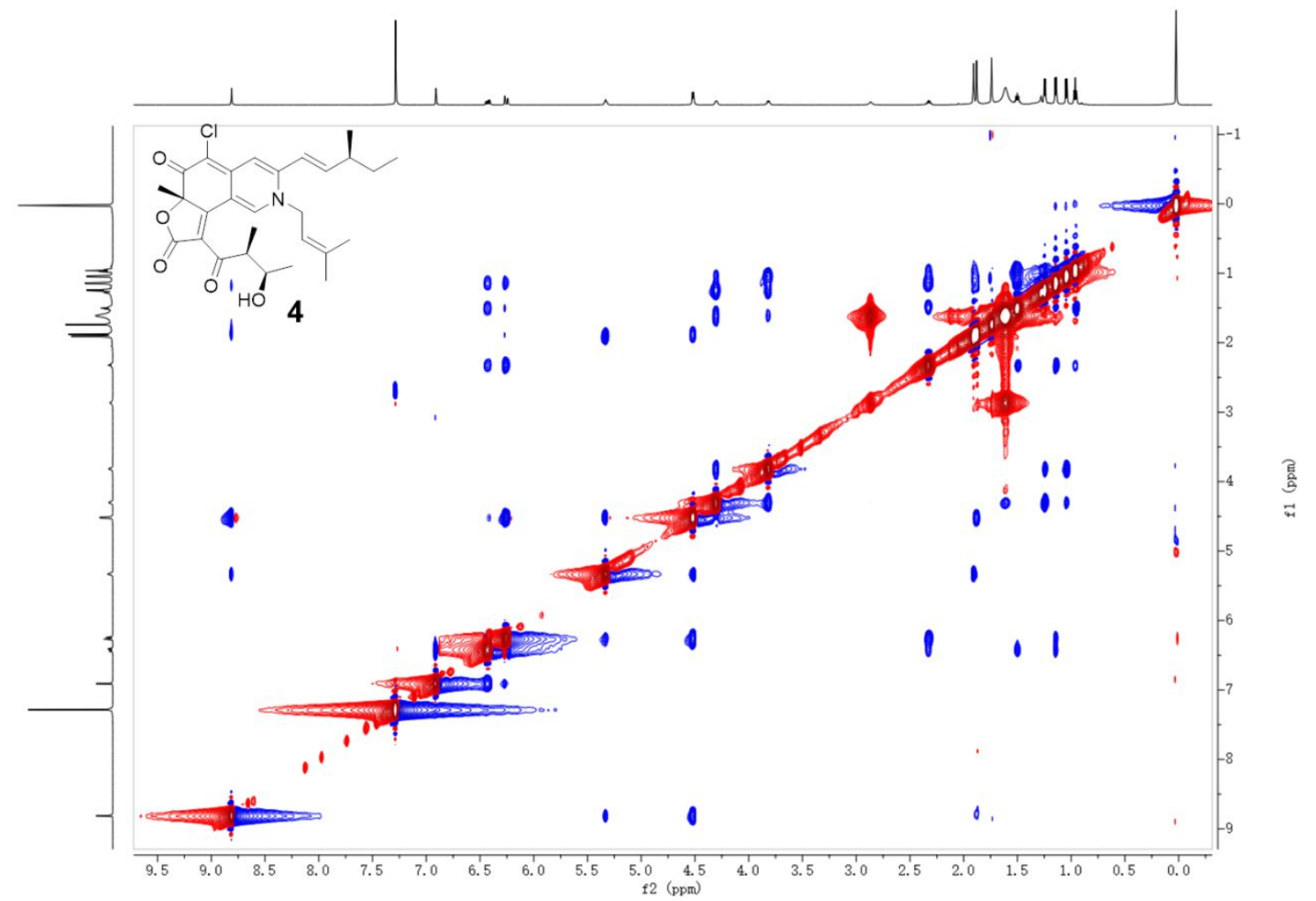

Figure S46. NOESY spectrum of compound 4 


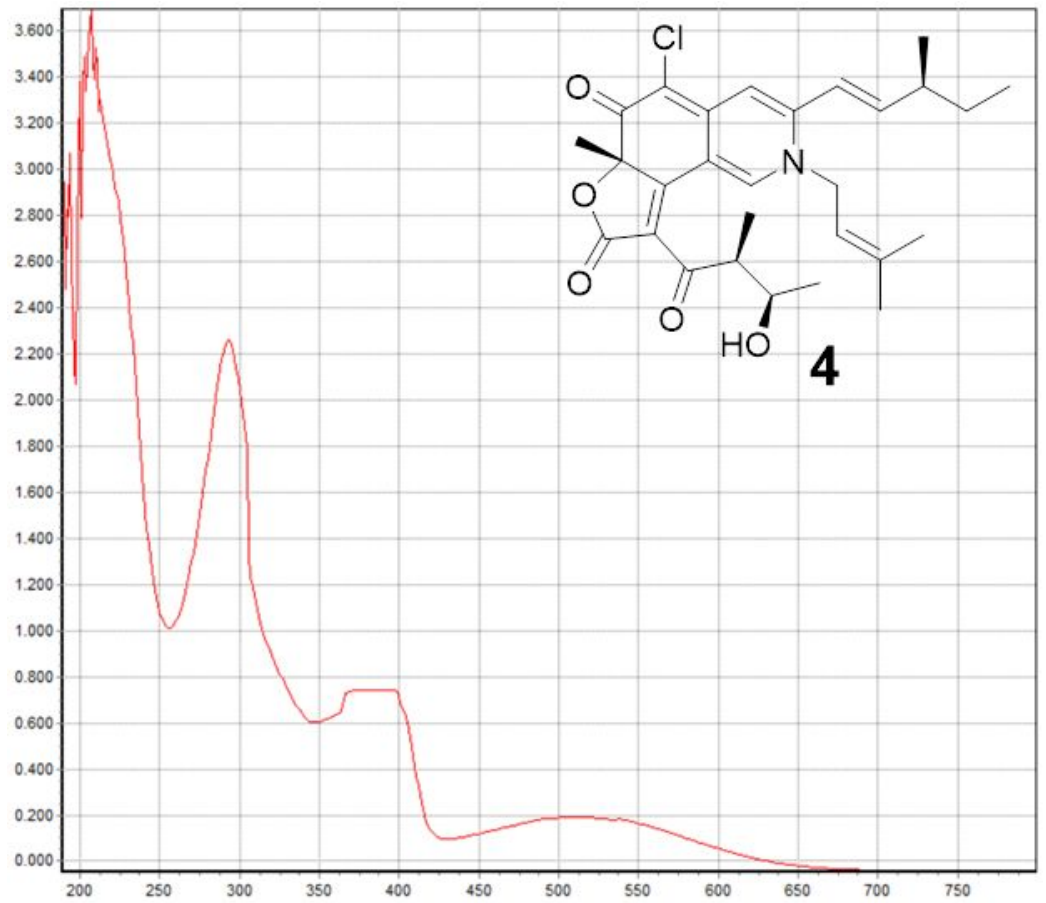

Figure S47. UV spectrum of compound 4

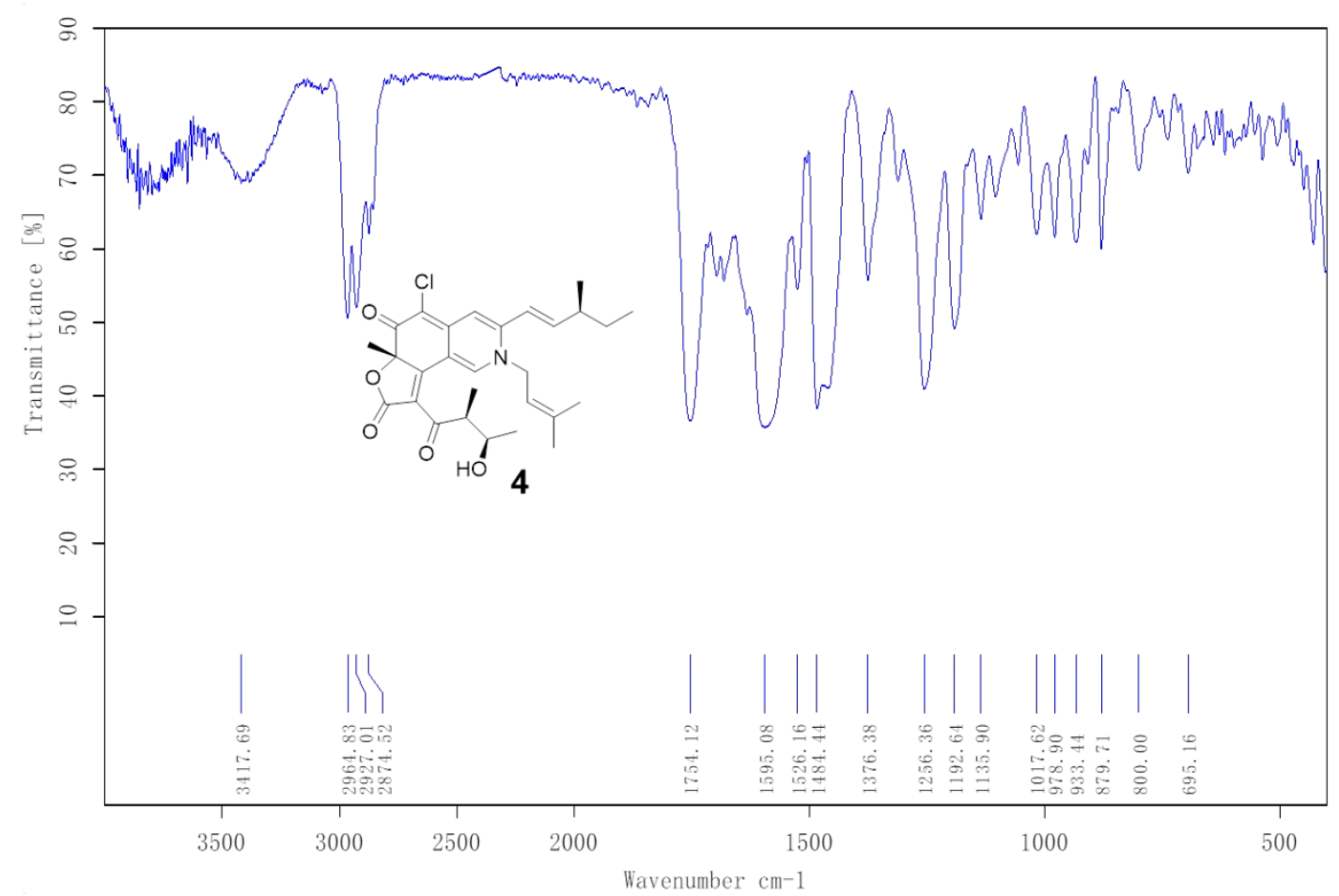

Figure S48. IR spectrum of compound 4 


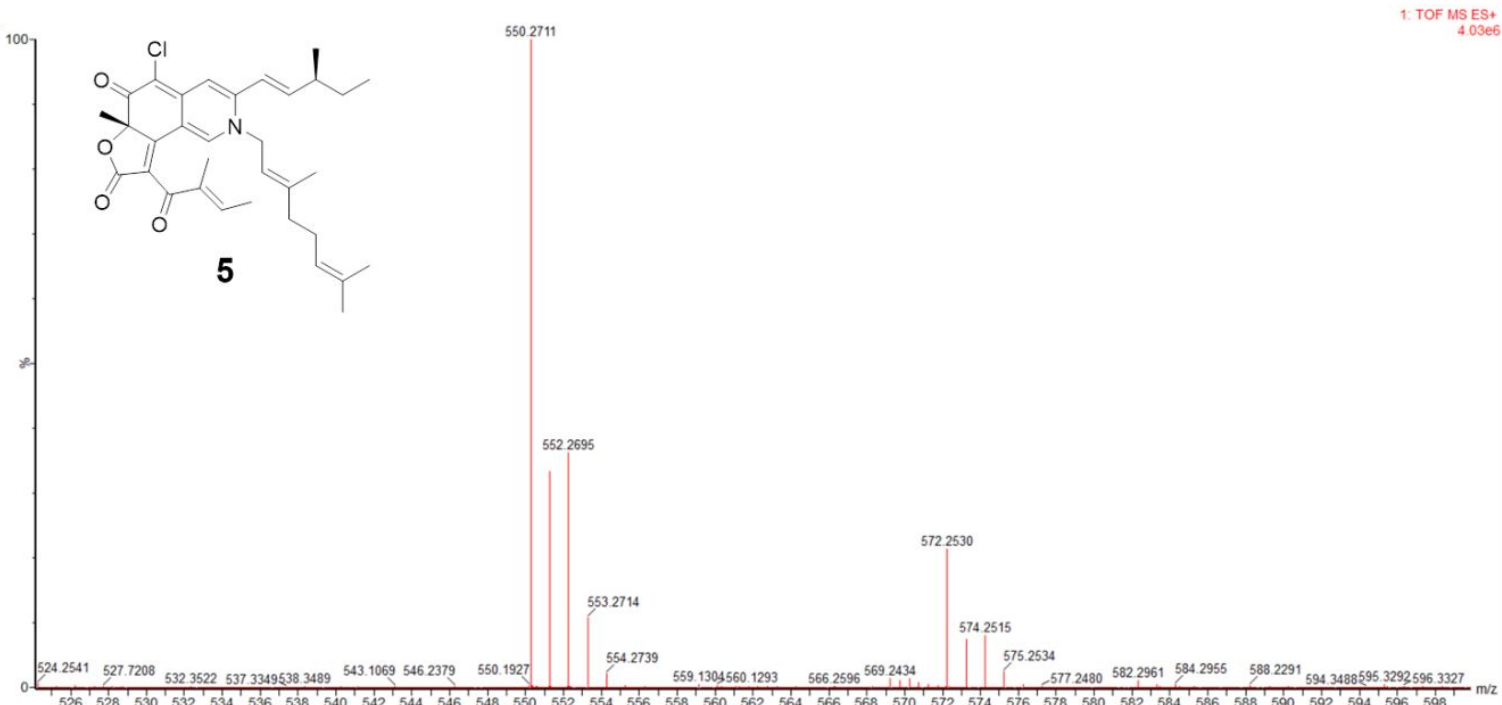

Figure S49. HRESIMS spectrum of compound 5

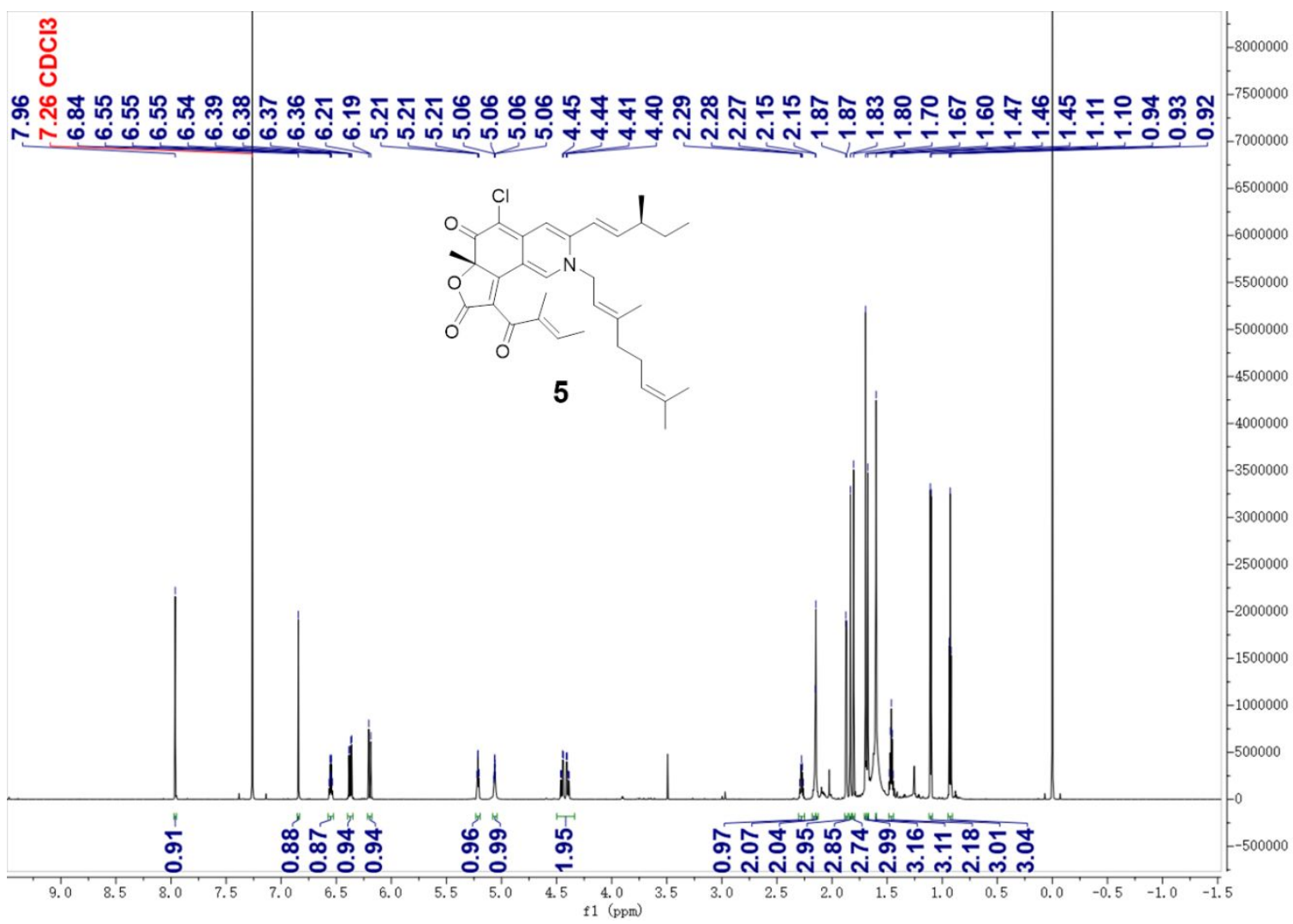

Figure S50. ${ }^{1} \mathrm{H}$ NMR spectrum of compound 5 


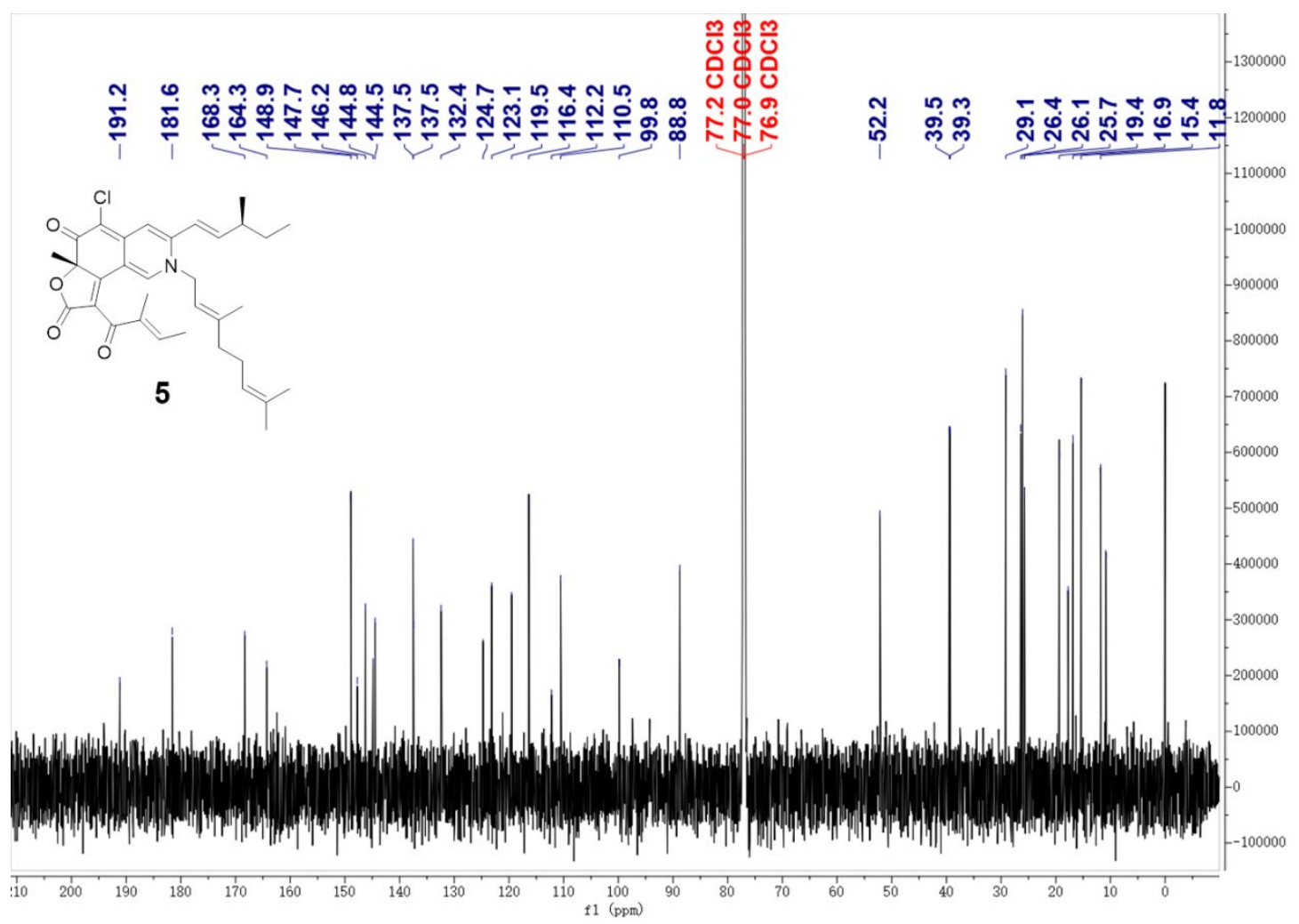

Figure S51. ${ }^{13} \mathrm{C}$ NMR spectrum of compound 5

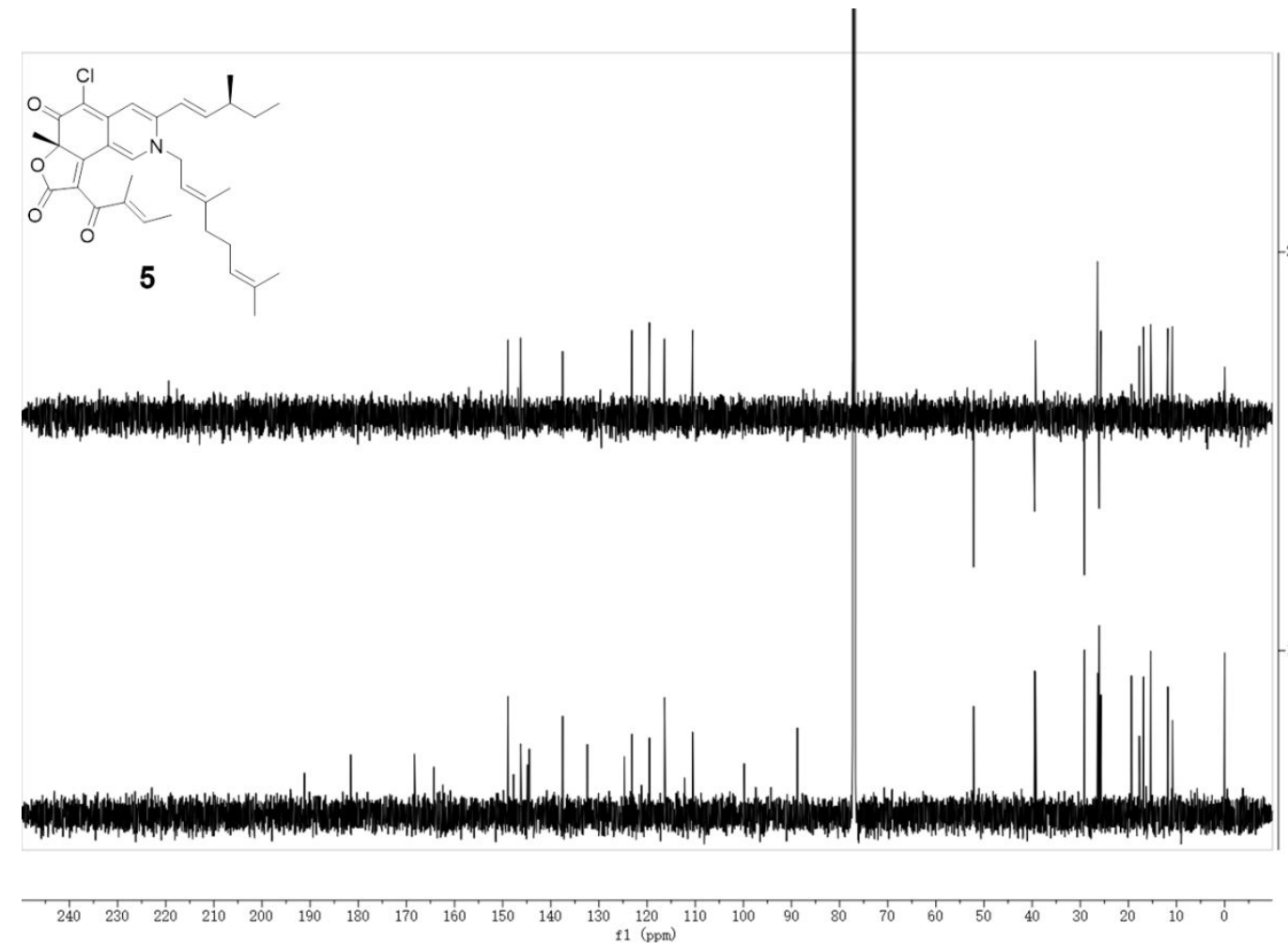

Figure S52. ${ }^{13} \mathrm{C} / \mathrm{DEPT}$ spectrum of compound 5 


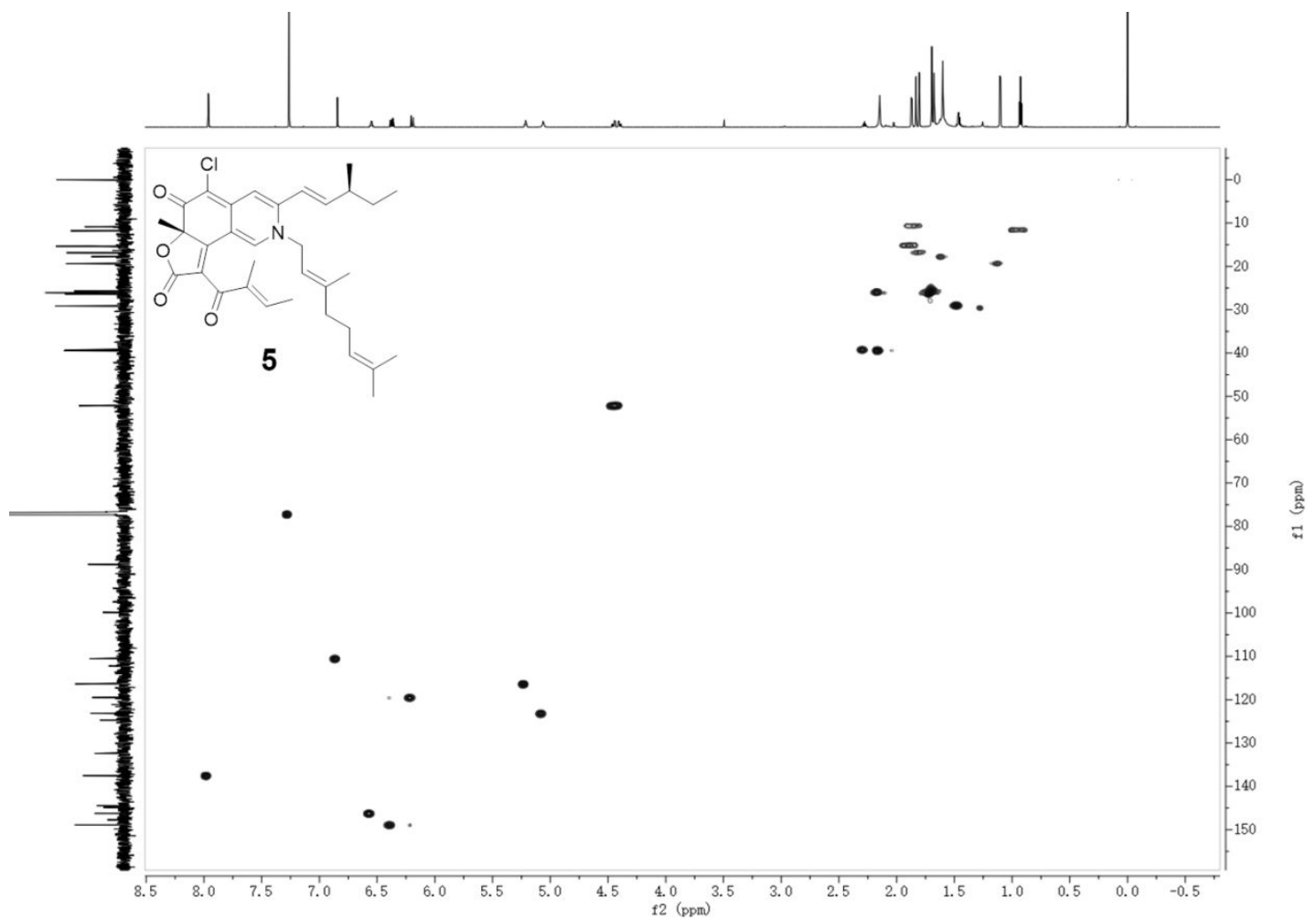

Figure S53. HSQC spectrum of compound 5

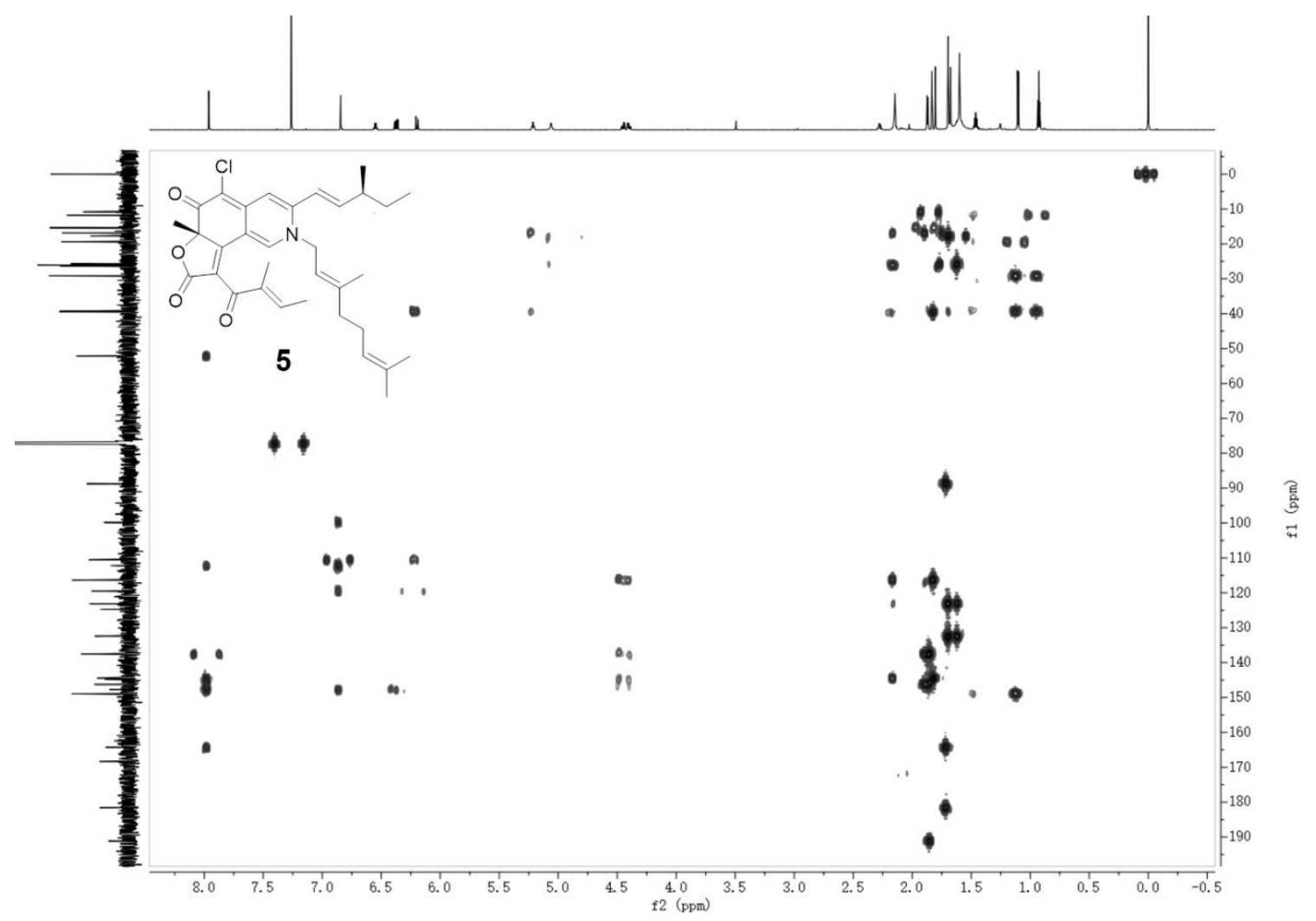

Figure S54. HMBC spectrum of compound 5 


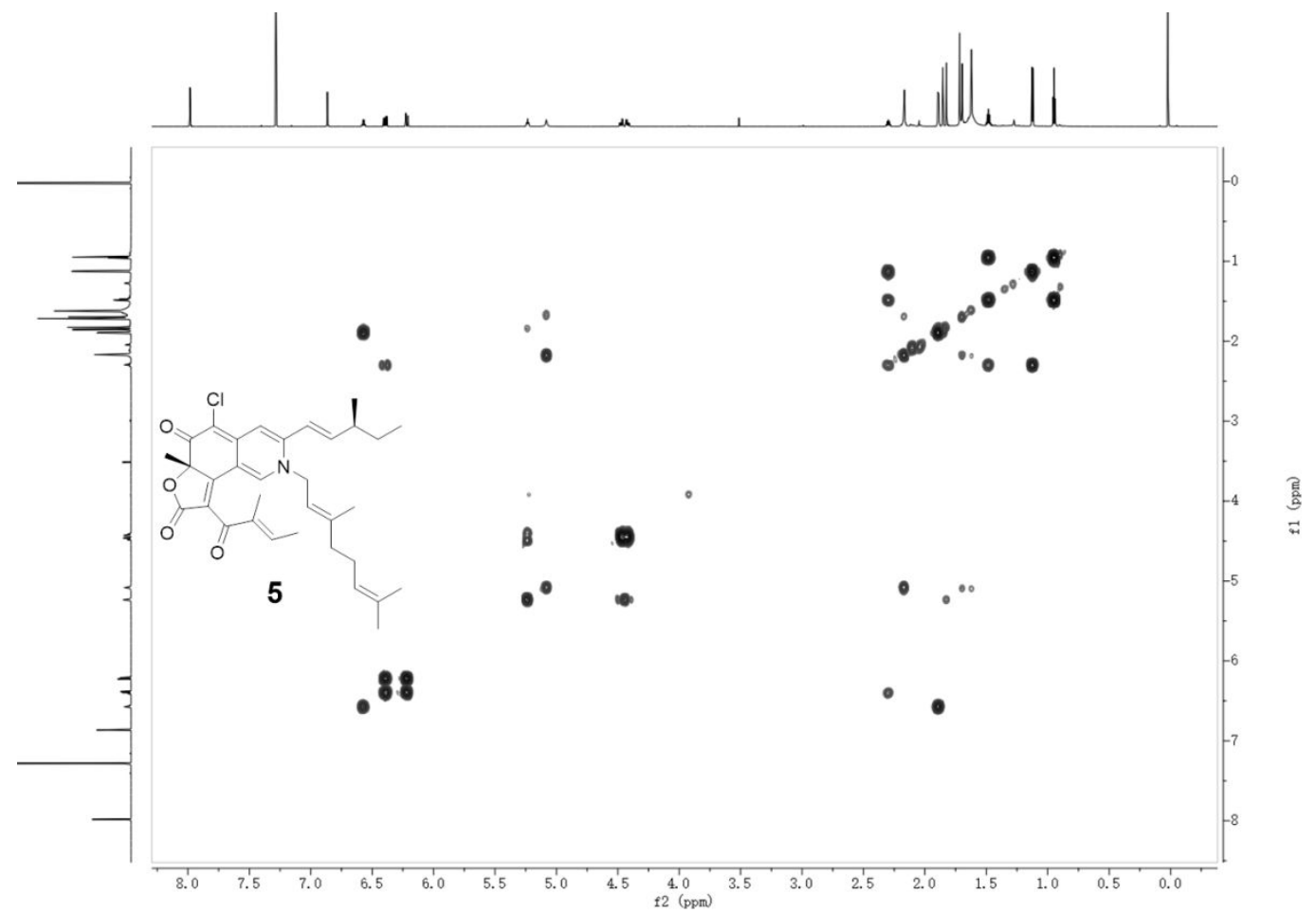

Figure S55. COSY spectrum of compound 5

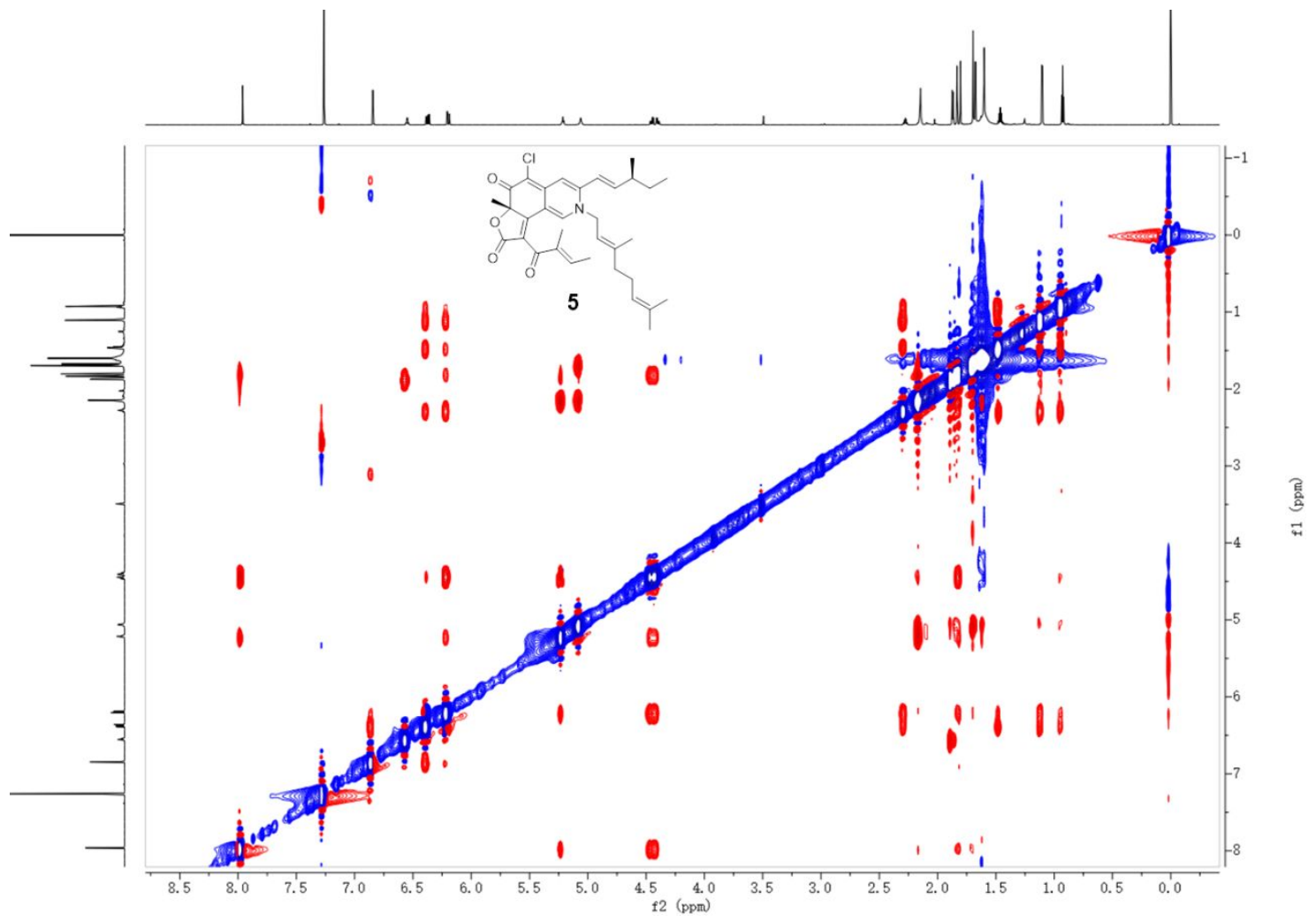

Figure S56. NOESY spectrum of compound $\mathbf{5}$ 


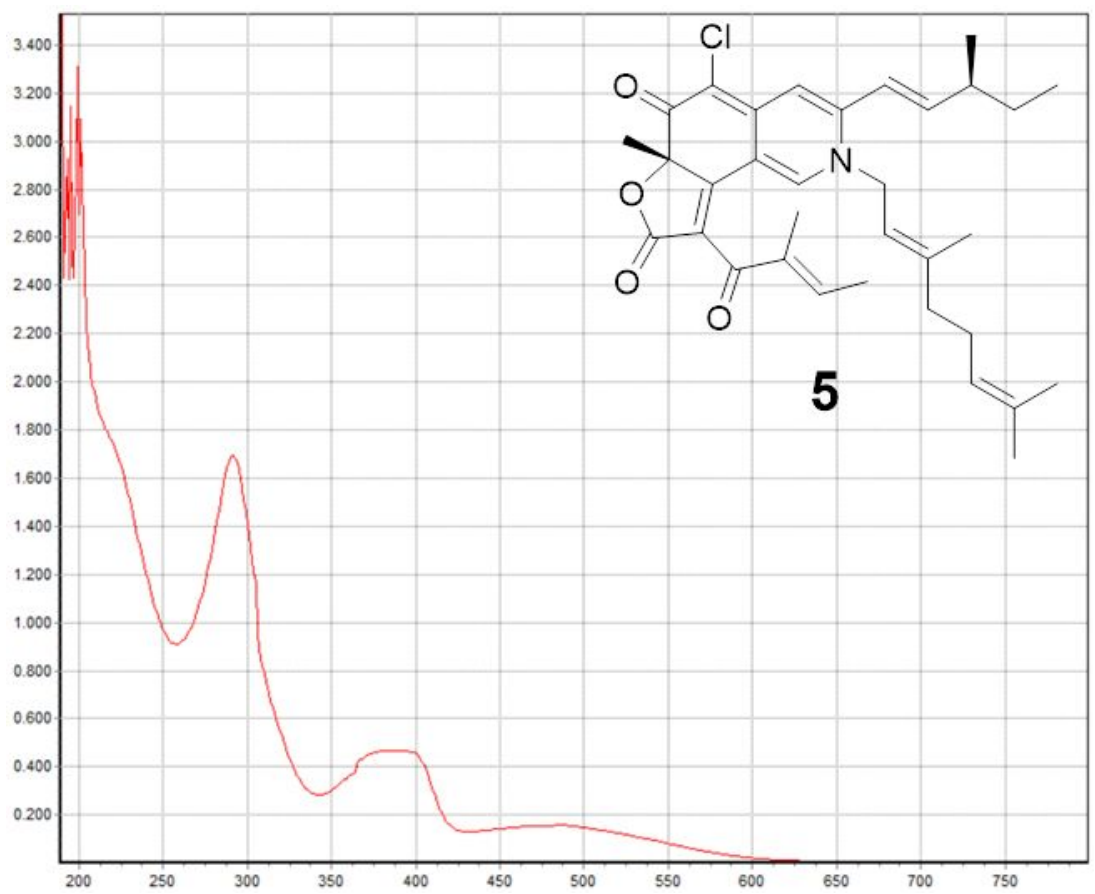

Figure S57. UV spectrum of compound 5

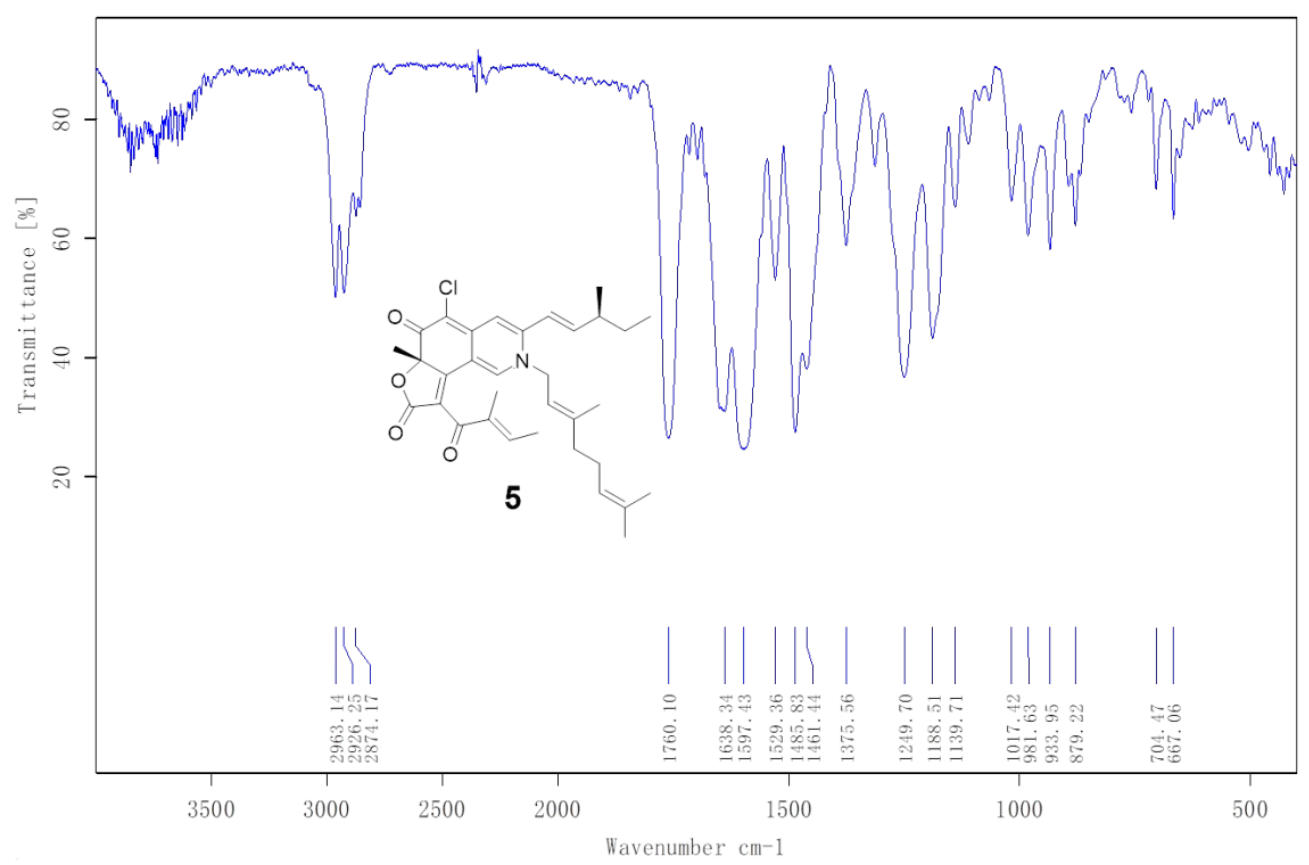

Figure S58. IR spectrum of compound 5 


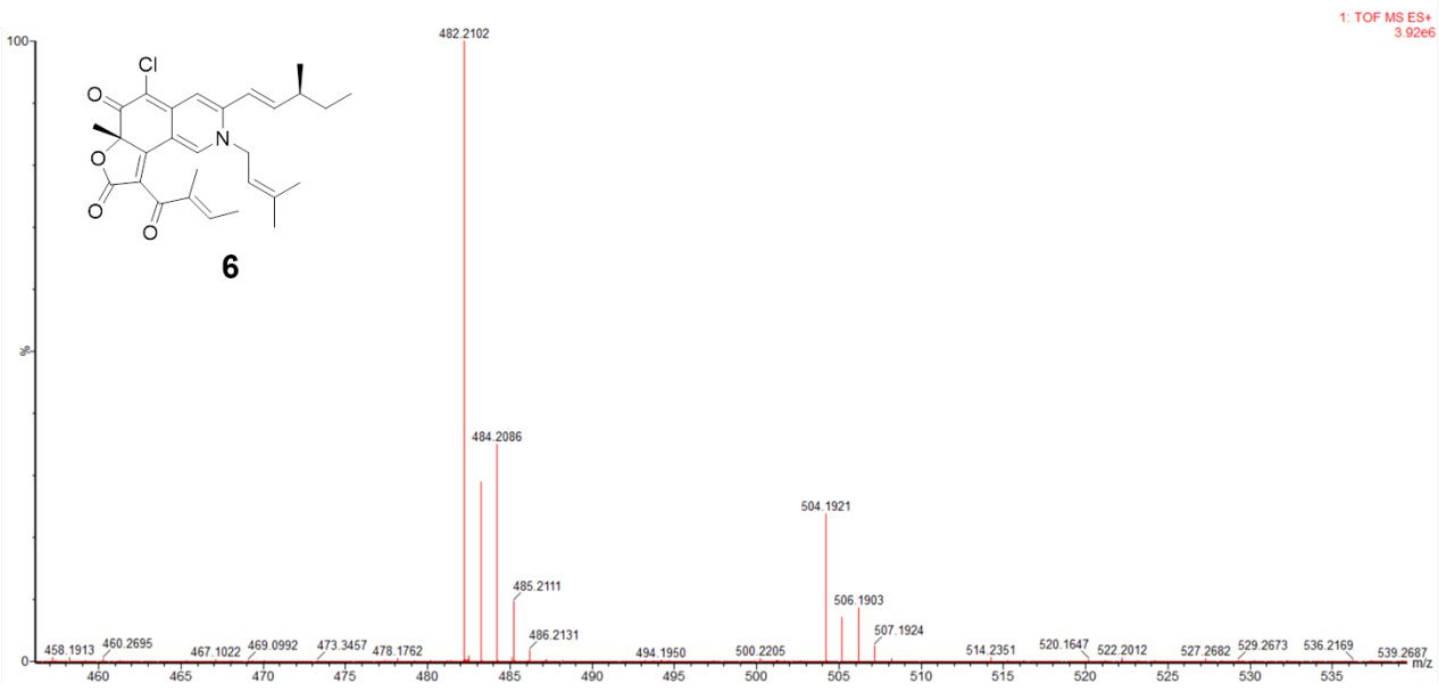

Figure S59. HRESIMS spectrum of compound 6

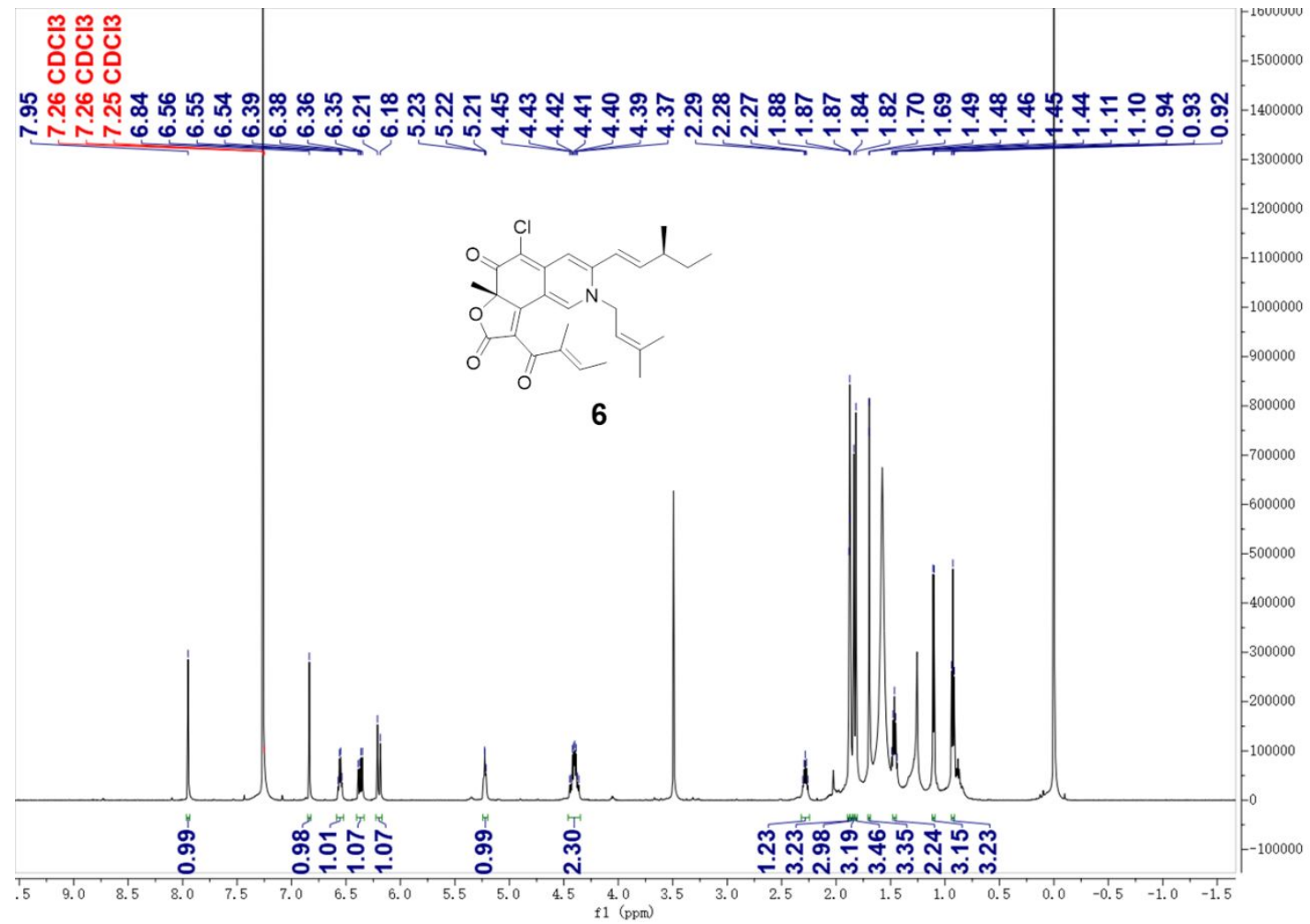

Figure S60. ${ }^{1} \mathrm{H}$ NMR spectrum of compound 6 


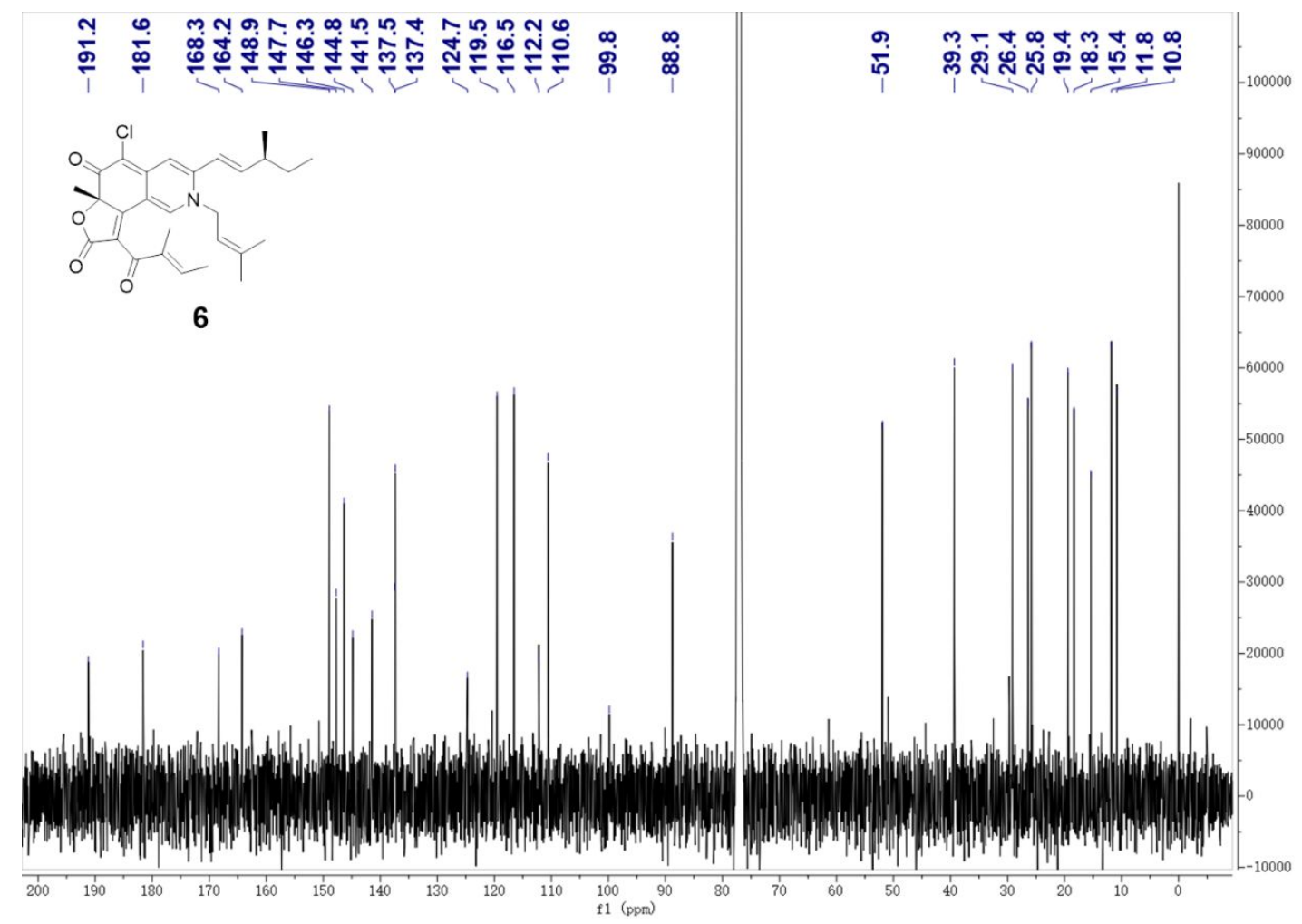

Figure S61. ${ }^{13} \mathrm{C}$ NMR spectrum of compound 6

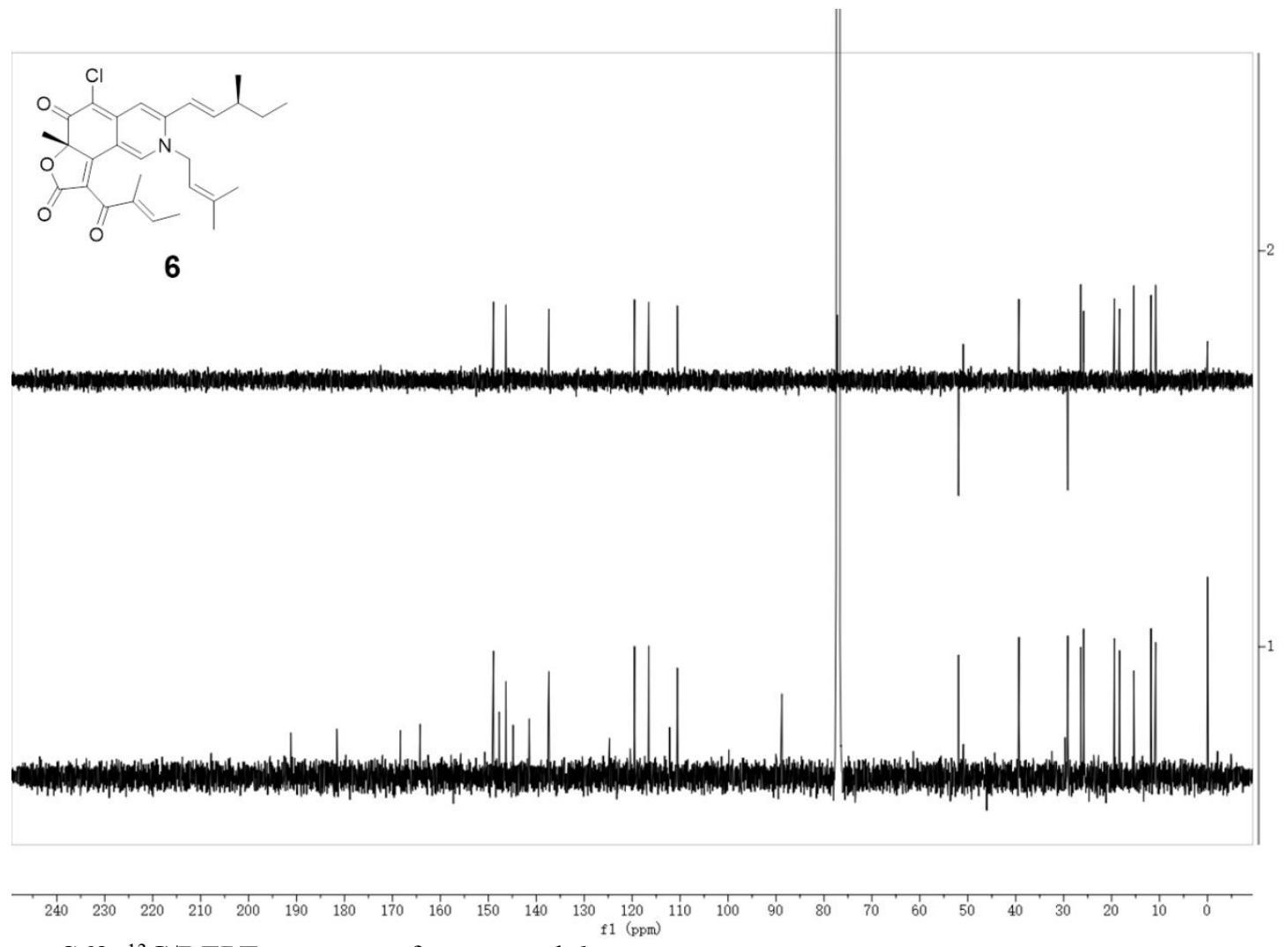

Figure S62. ${ }^{13} \mathrm{C} / \mathrm{DEPT}$ spectrum of compound 6 


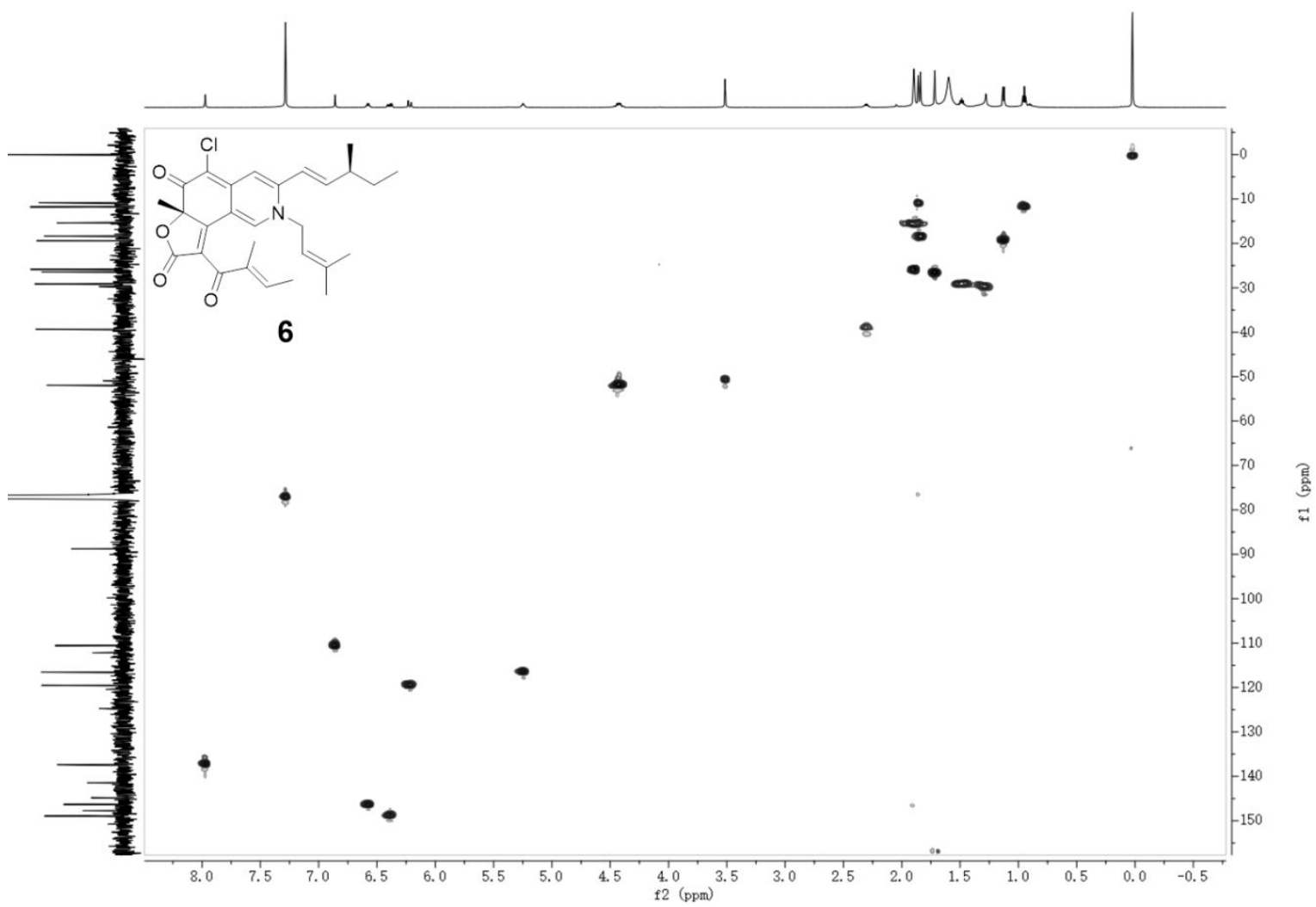

Figure S63. HSQC spectrum of compound 6

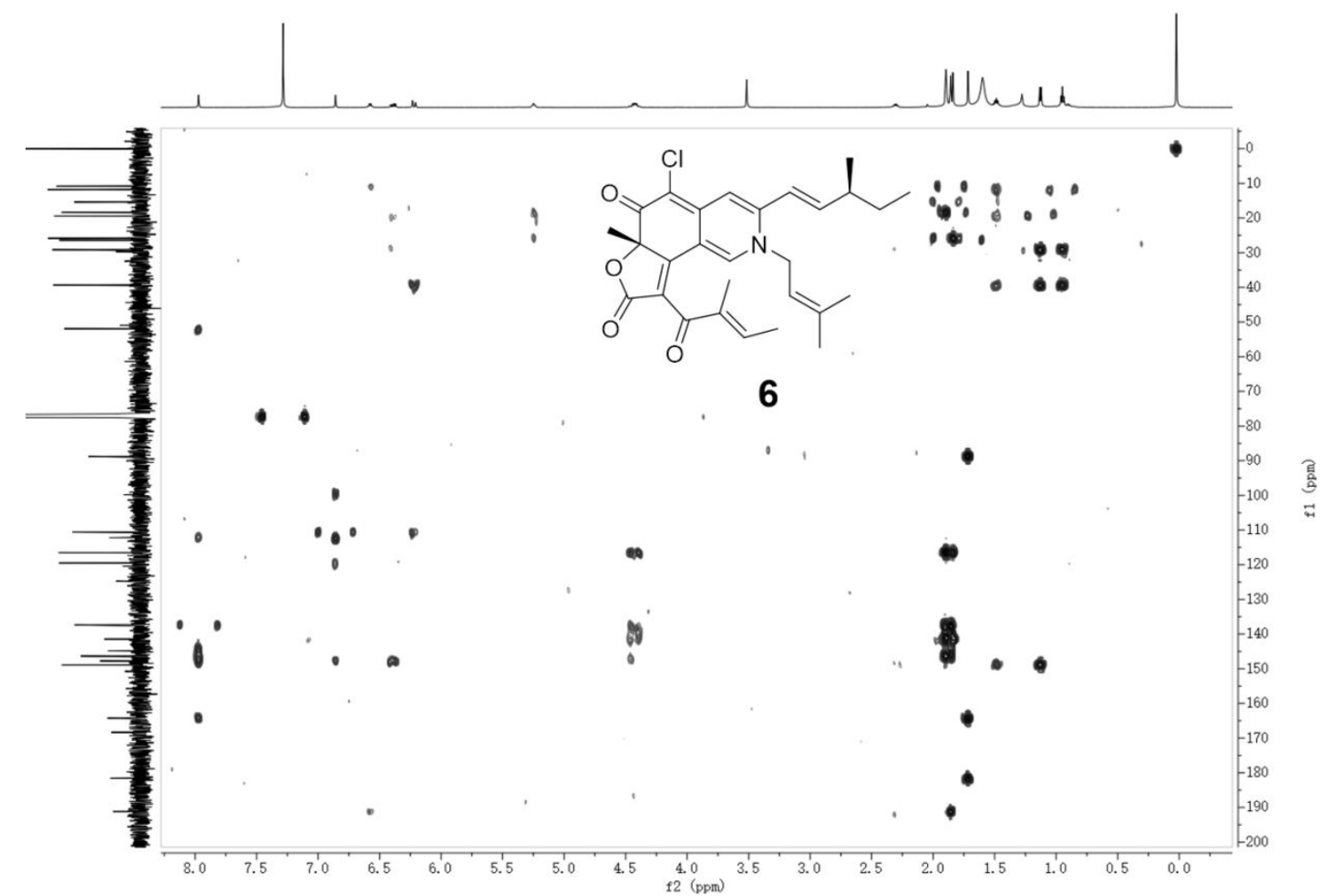

Figure S64. HMBC spectrum of compound 6 


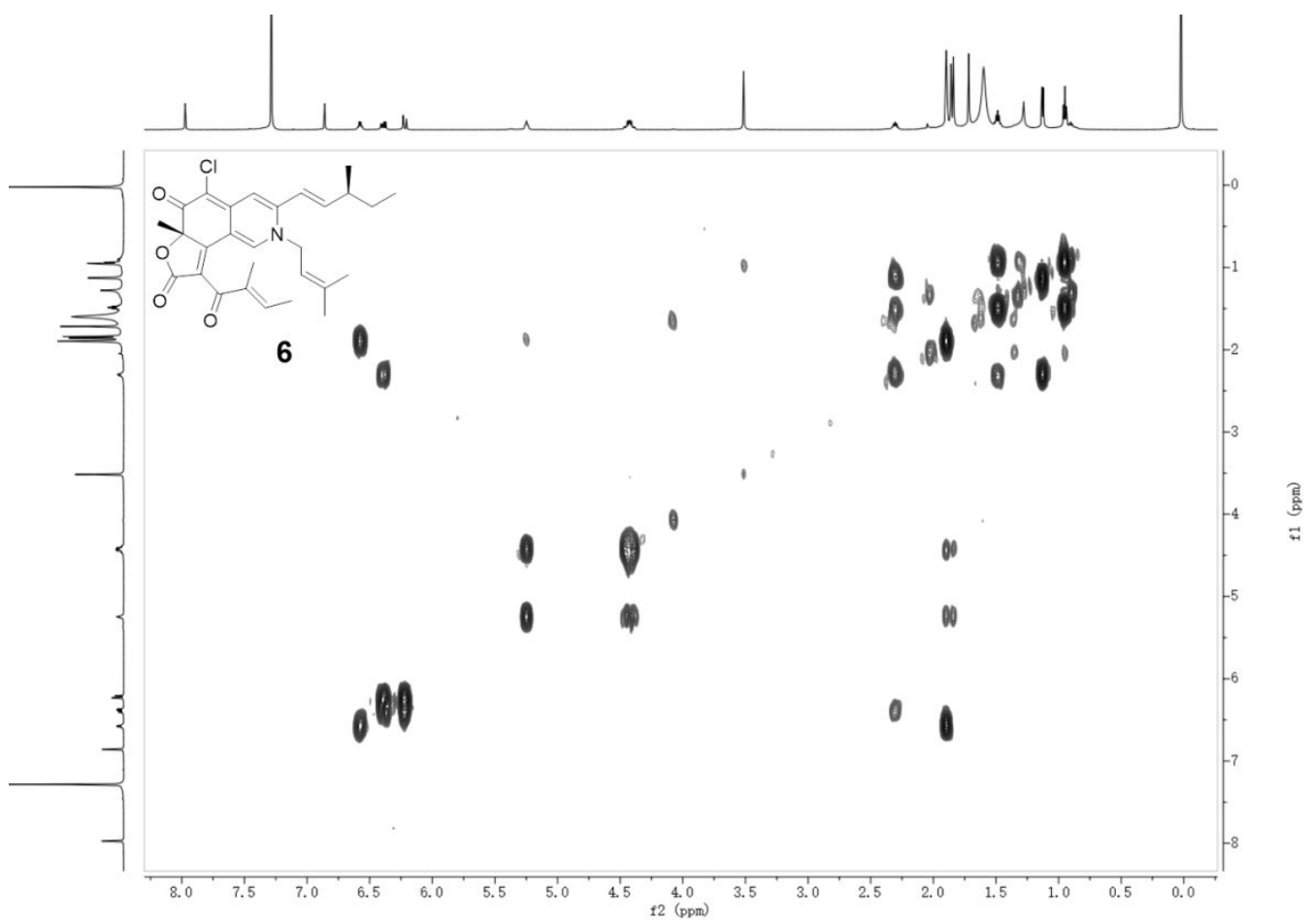

Figure S65. COSY spectrum of compound 6

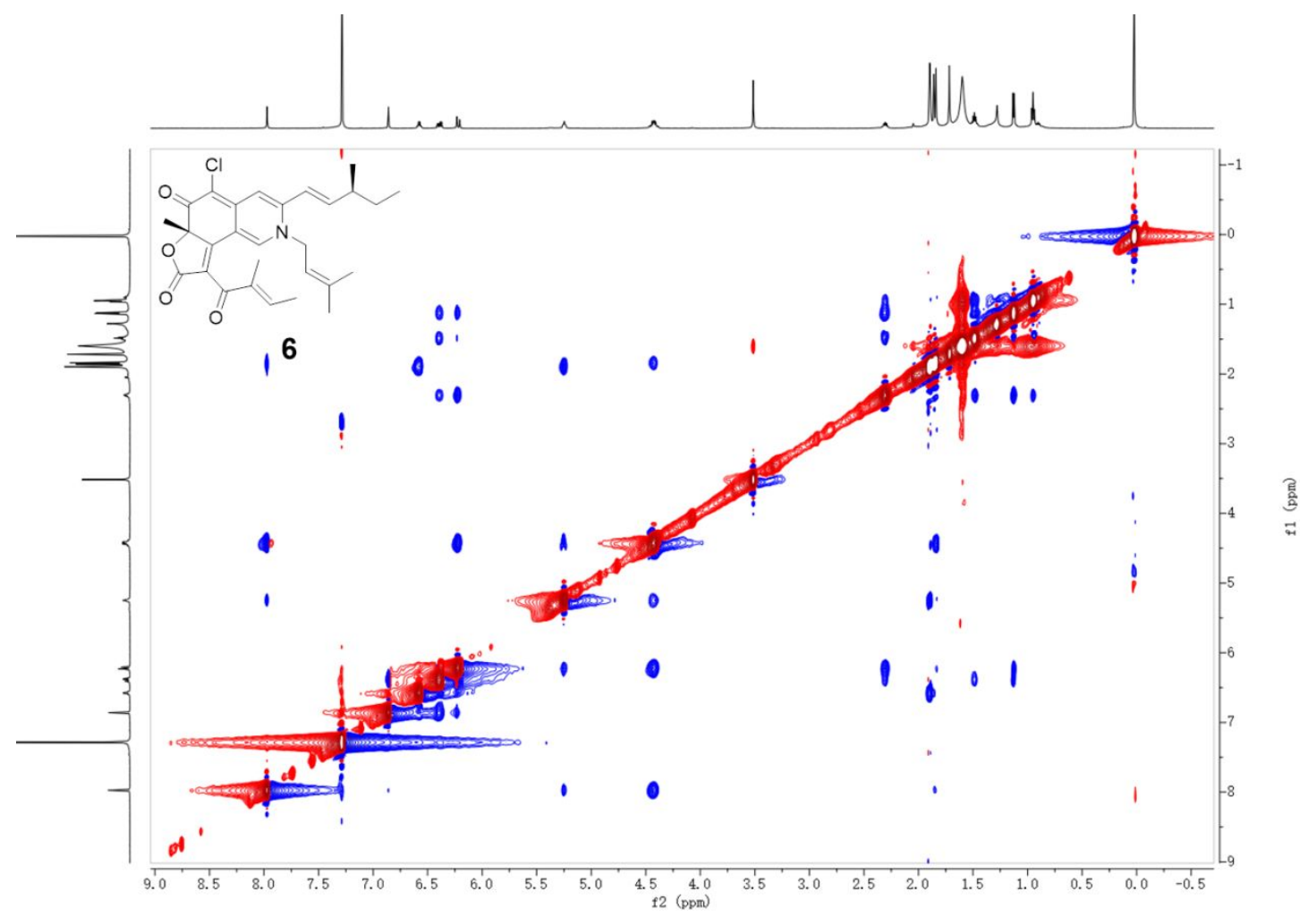

Figure S66. NOESY spectrum of compound 6 


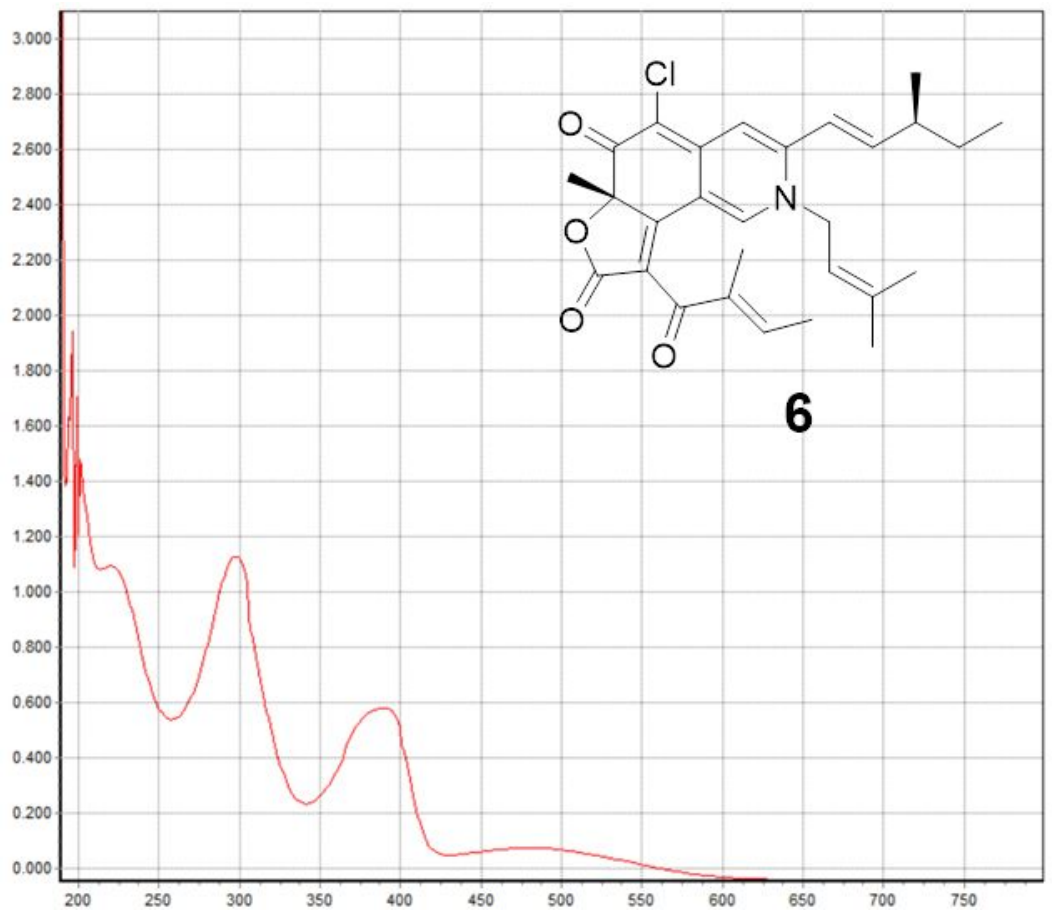

Figure S67. UV spectrum of compound 6

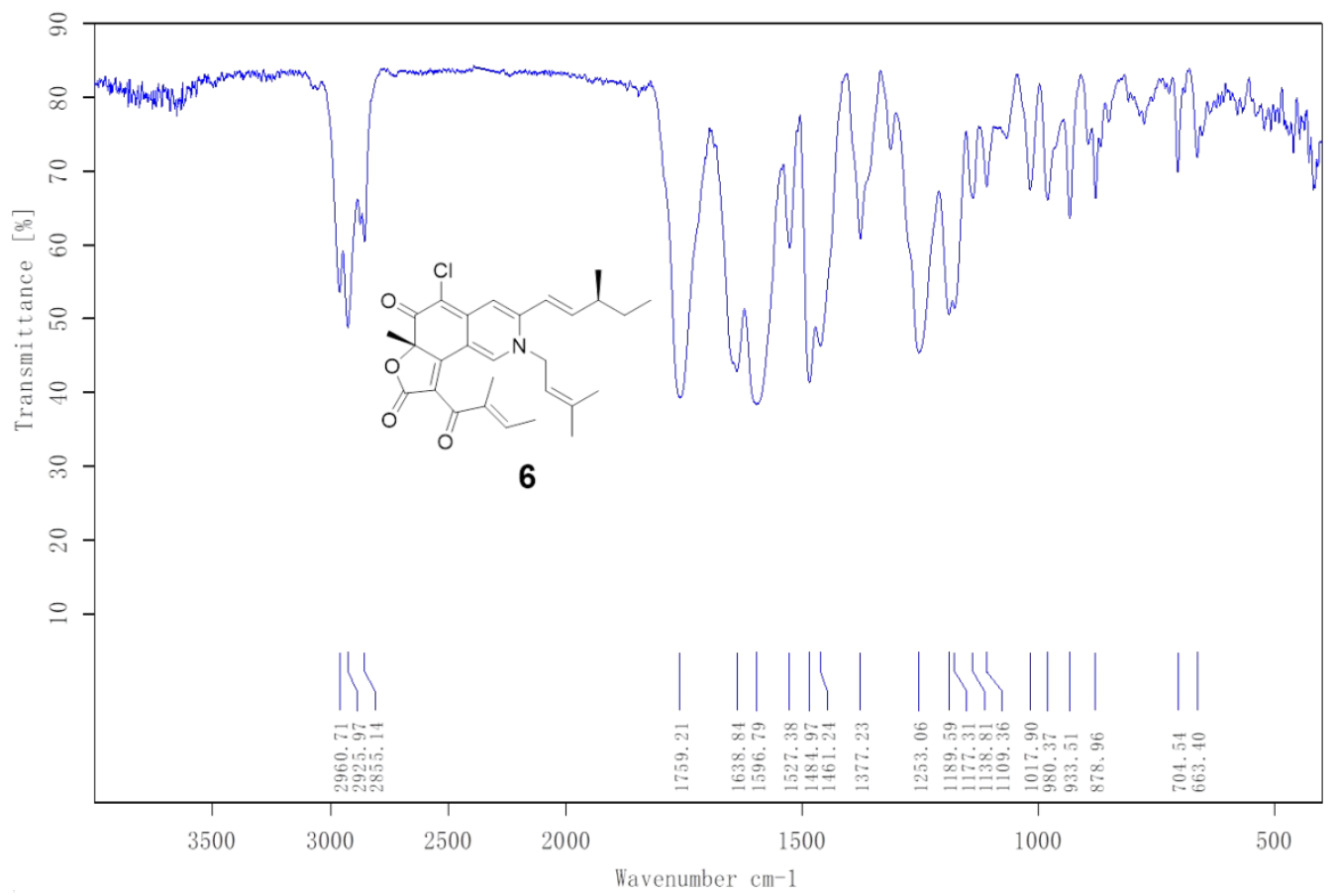

Figure S68. IR spectrum of compound 6 


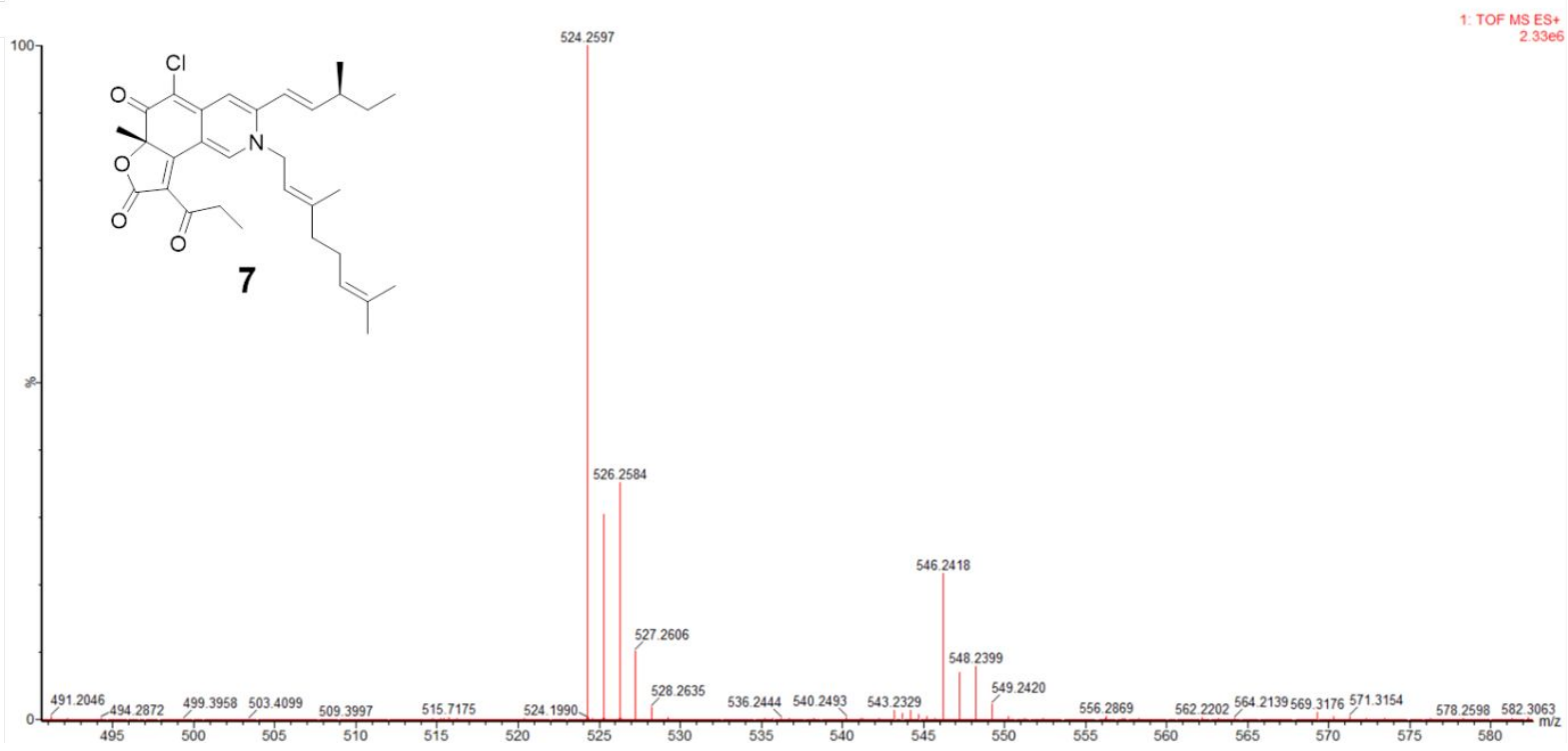

Figure S69. HRESIMS spectrum of compound 7

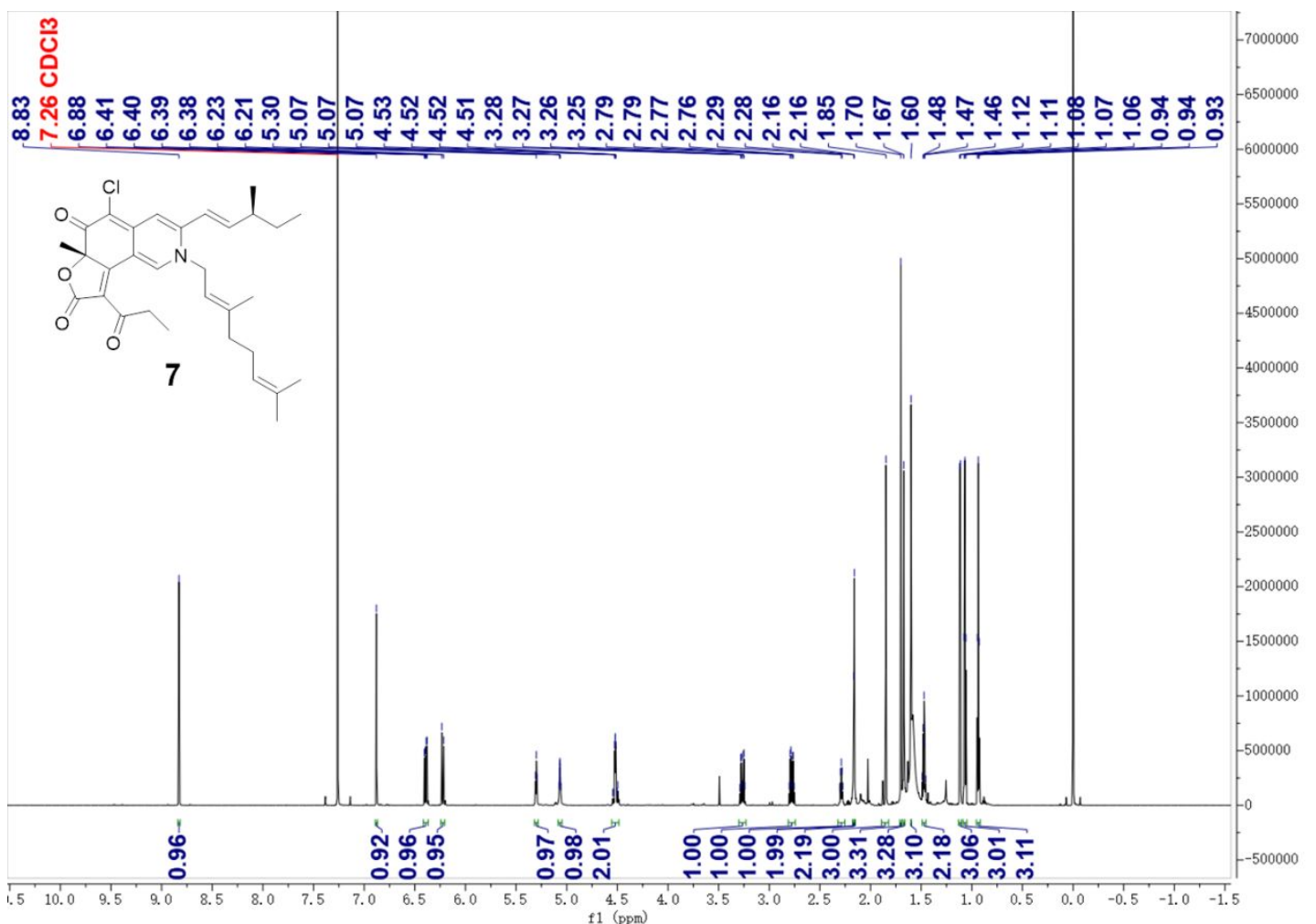

Figure S70. ${ }^{1} \mathrm{H}$ NMR spectrum of compound 7 


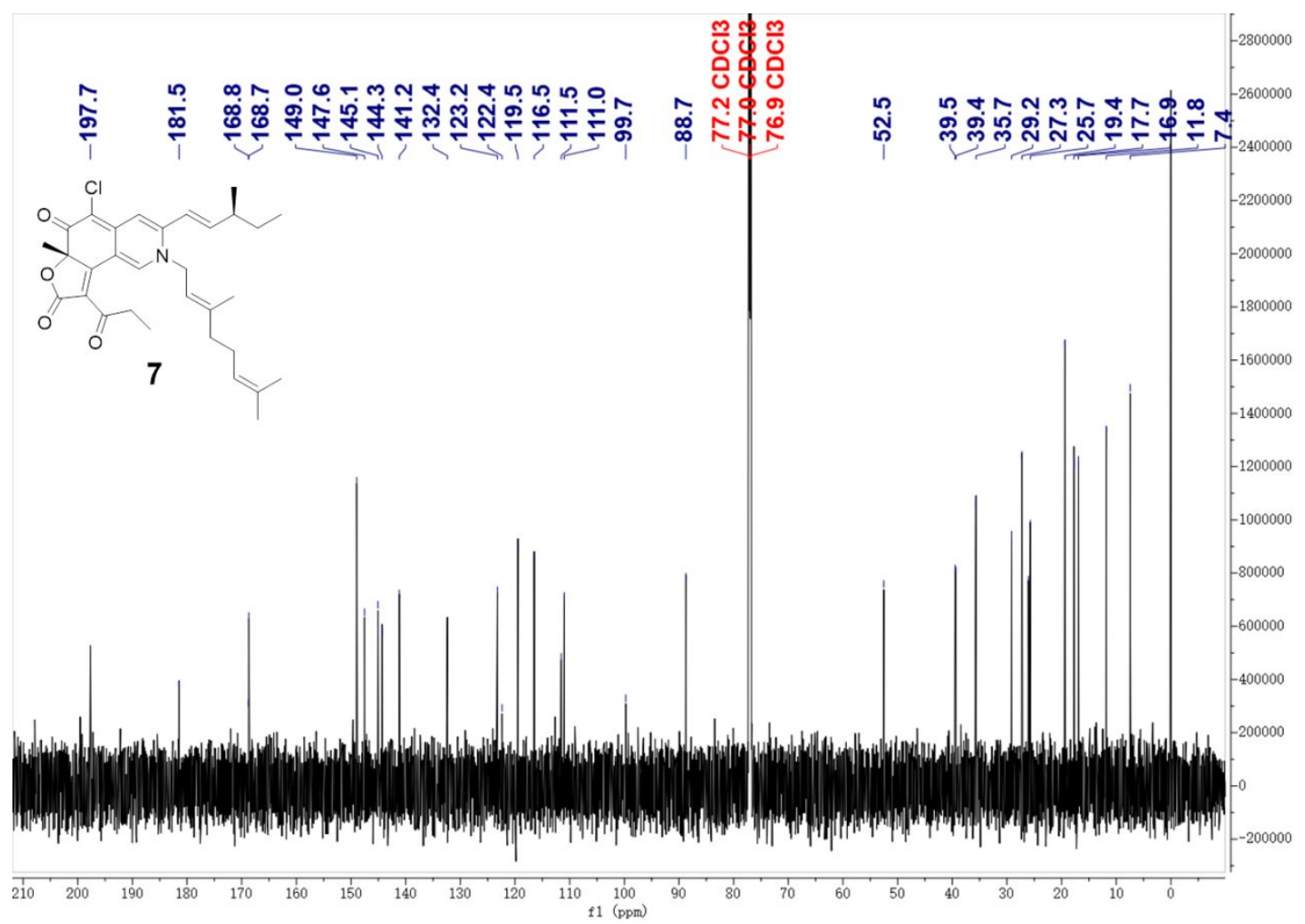

Figure S71. ${ }^{13} \mathrm{C}$ NMR spectrum of compound 7

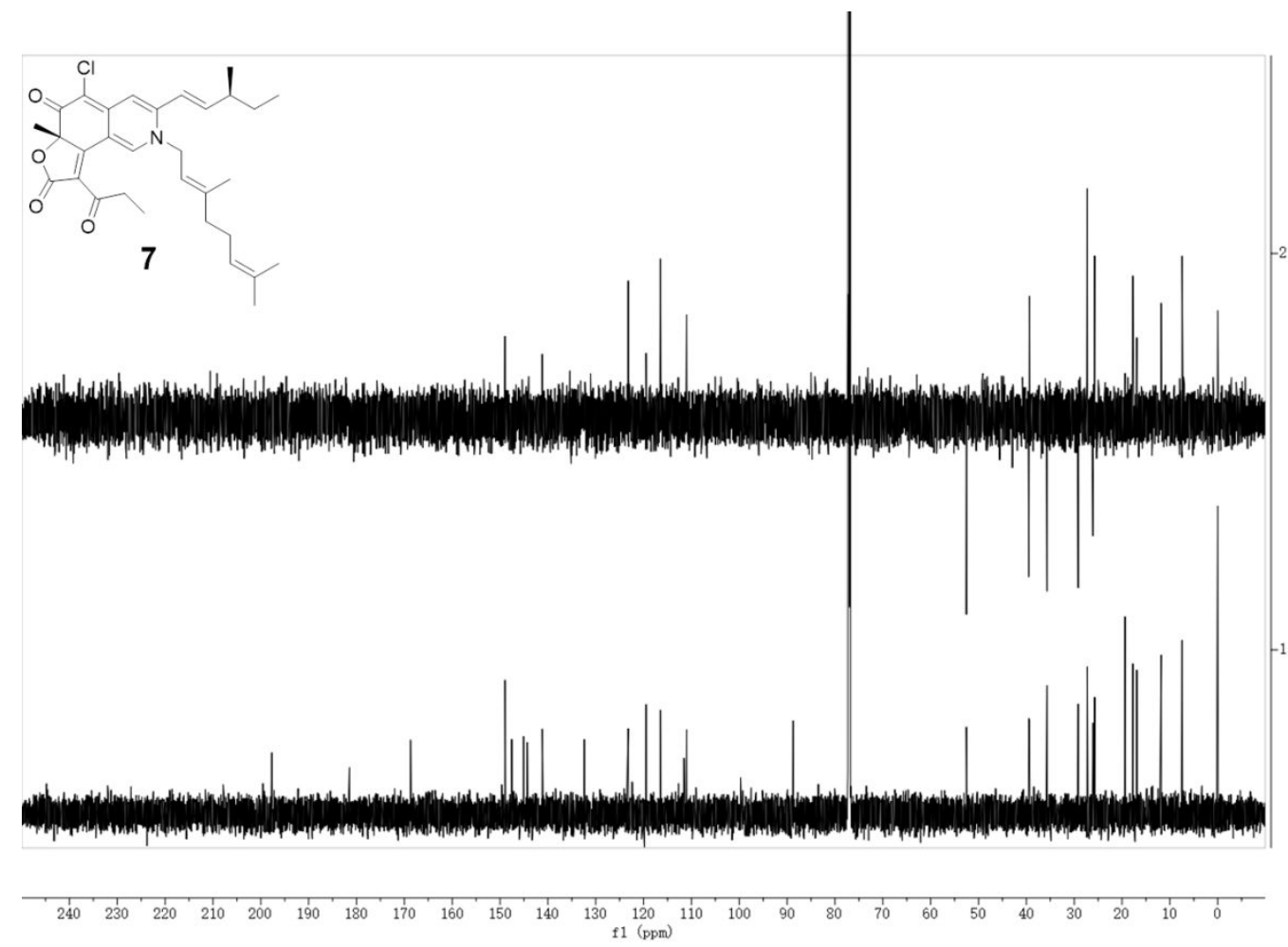

Figure S72. ${ }^{13} \mathrm{C} / \mathrm{DEPT}$ spectrum of compound 7 


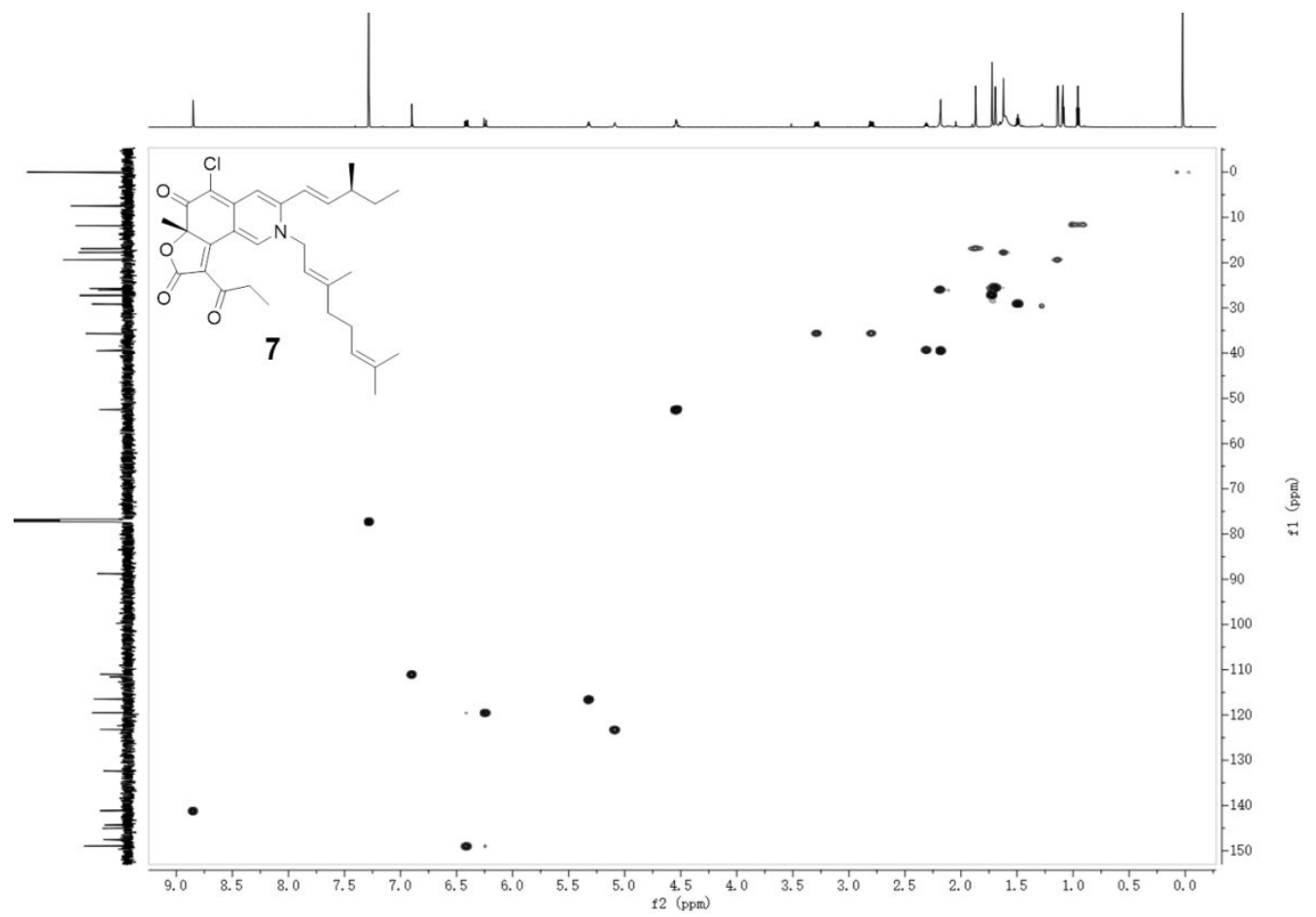

Figure S73. HSQC spectrum of compound 7

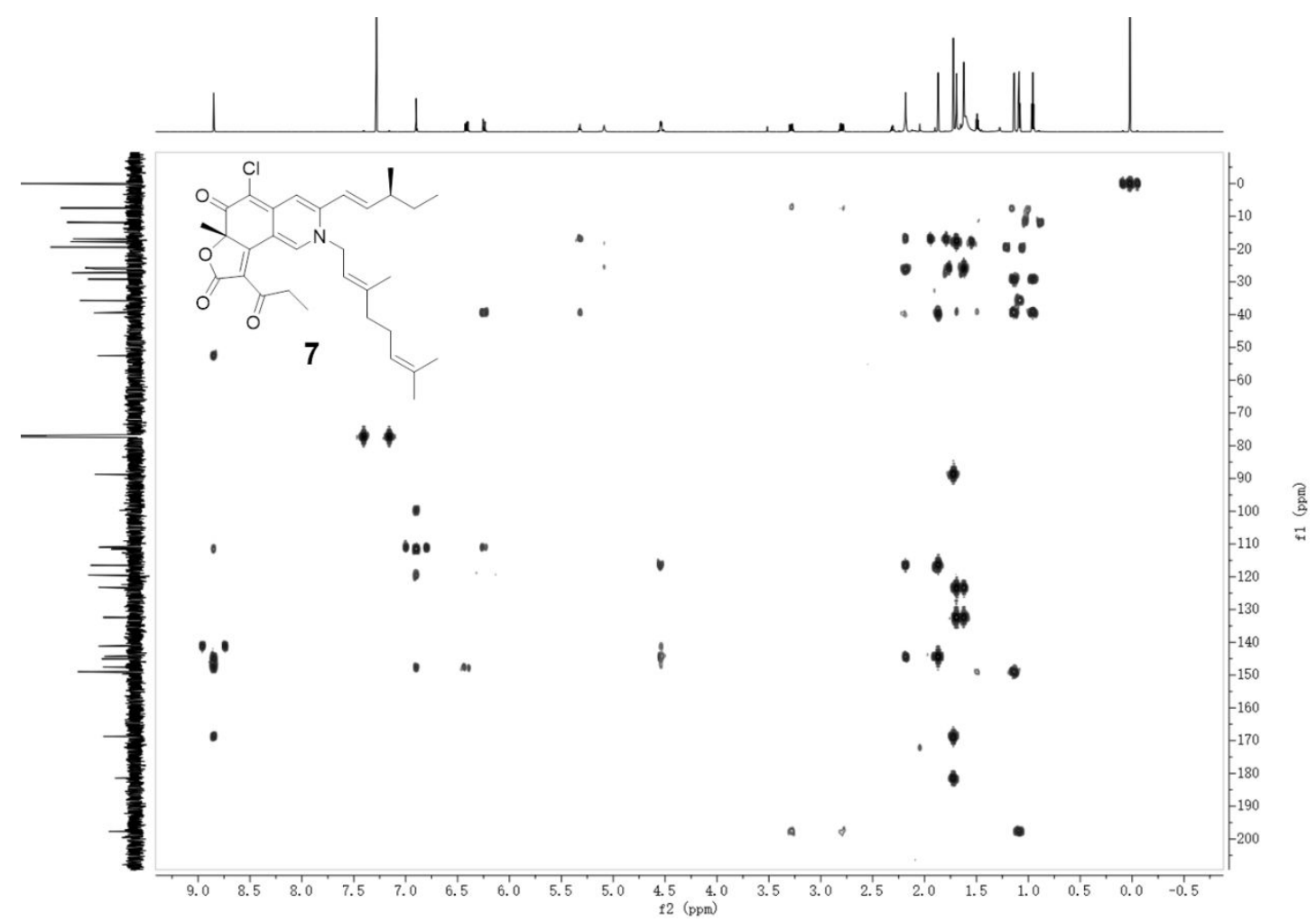

Figure S74. HMBC spectrum of compound 7 


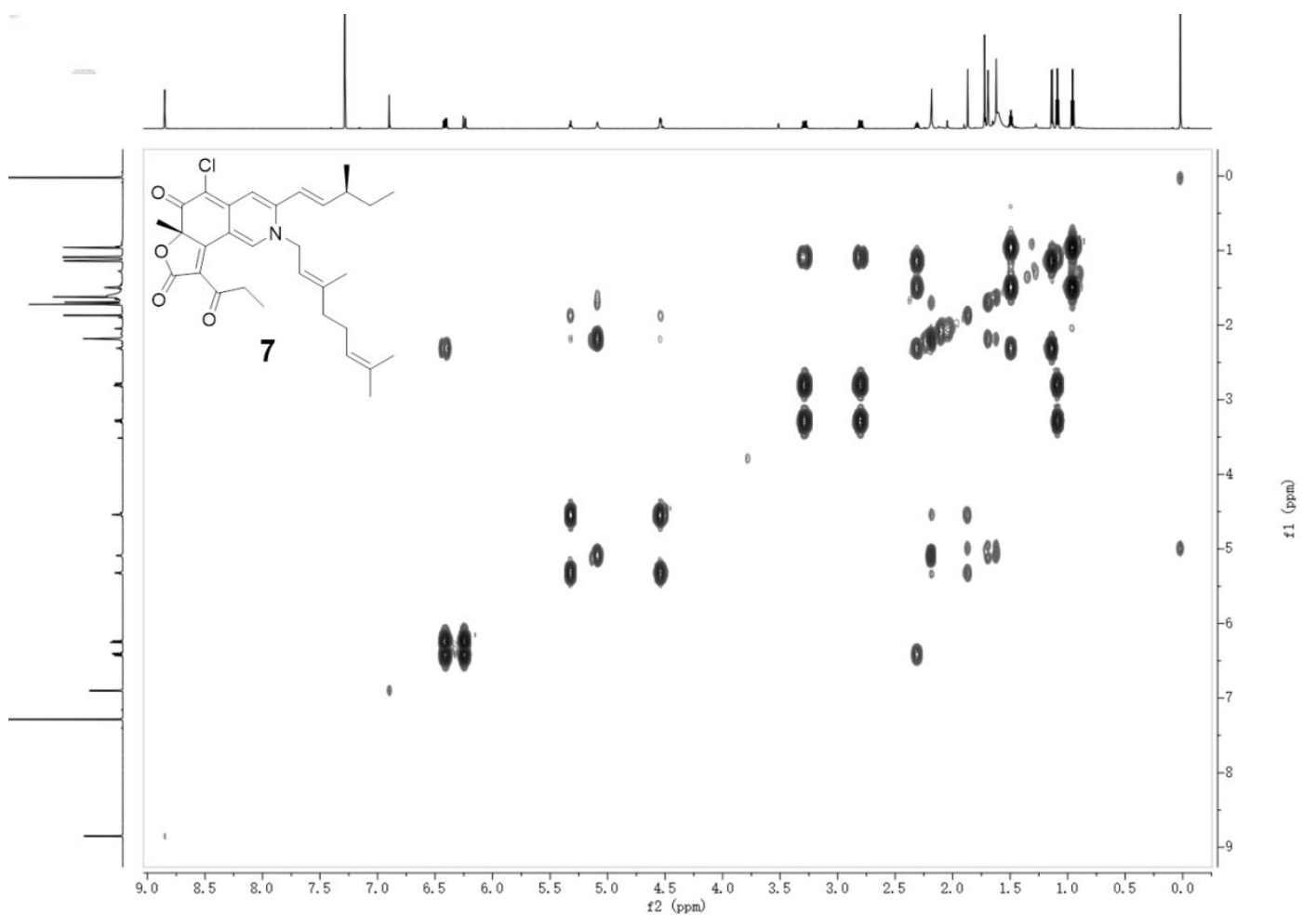

Figure S75. COSY spectrum of compound 7

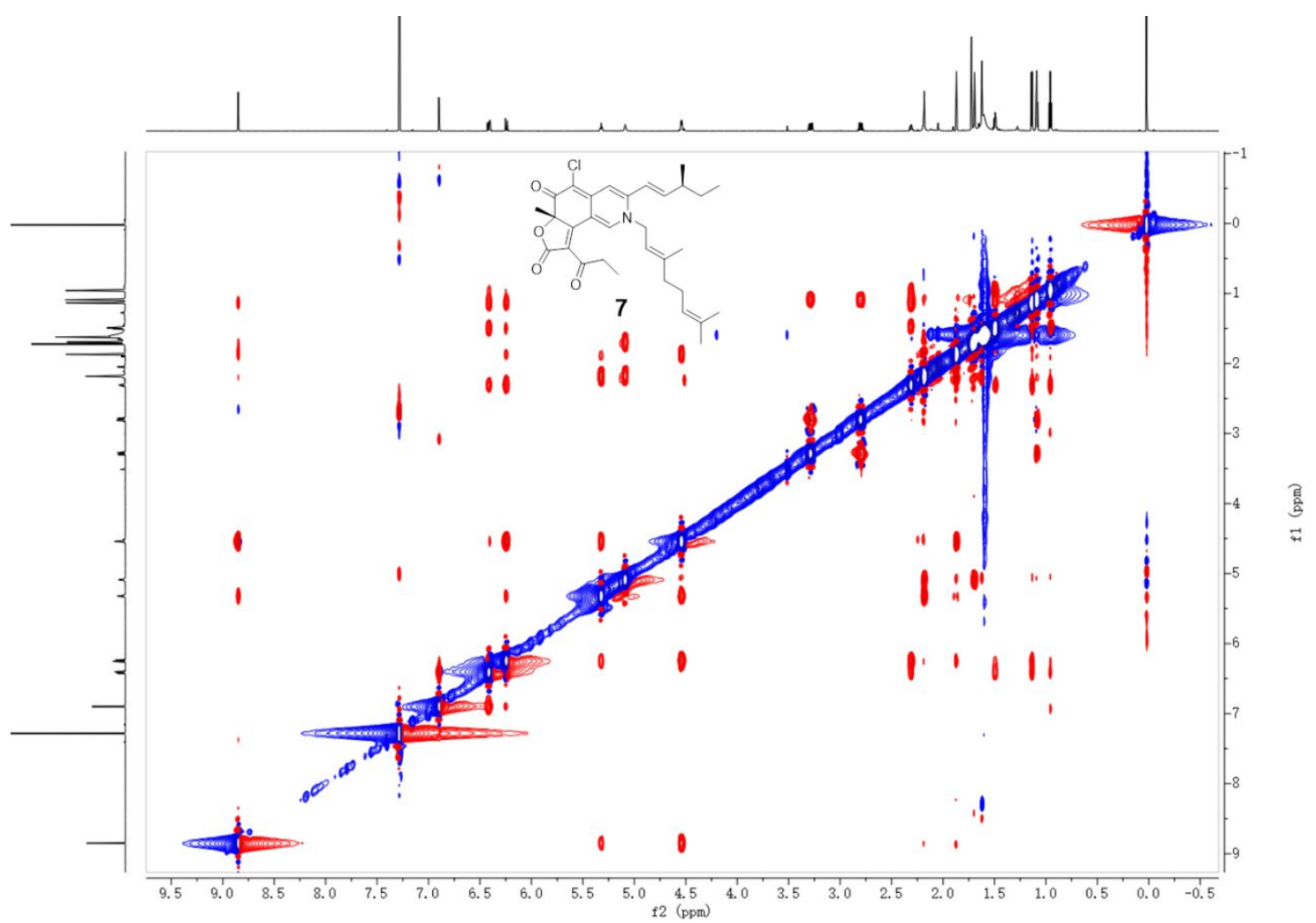

Figure S76. NOESY spectrum of compound 7 


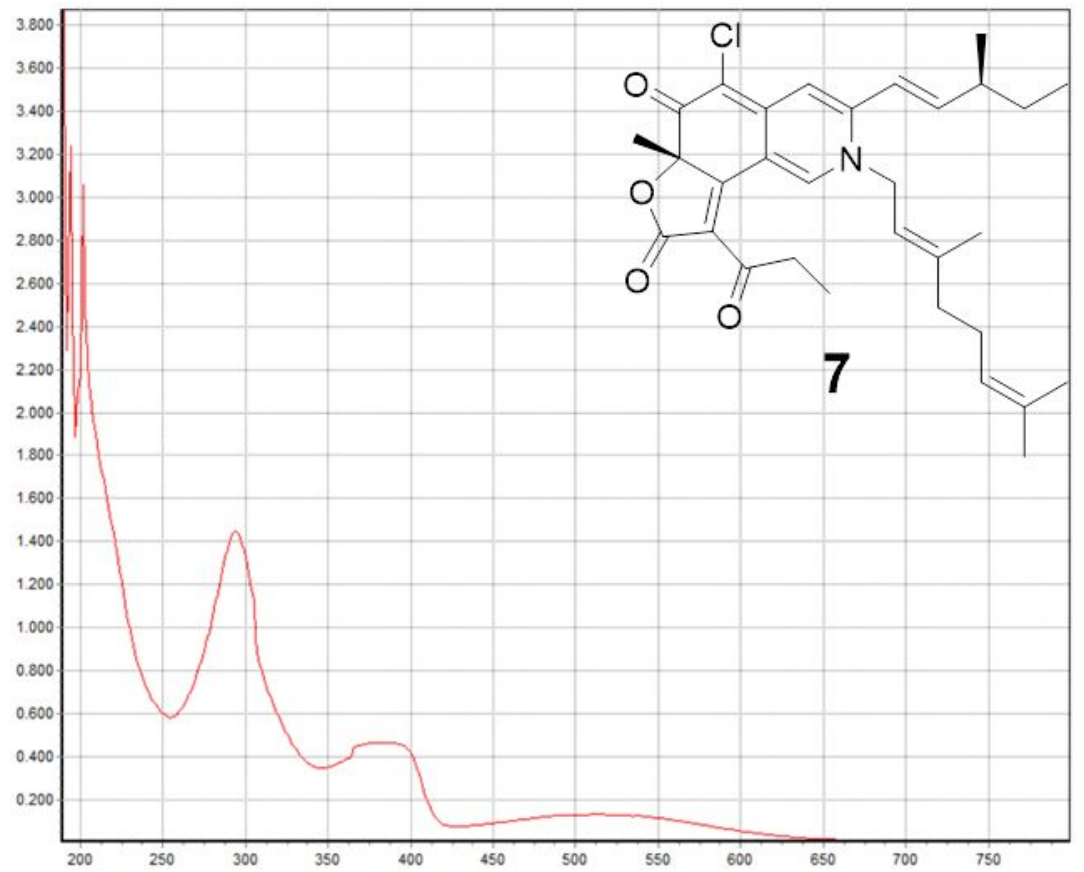

Figure S77. UV spectrum of compound 7

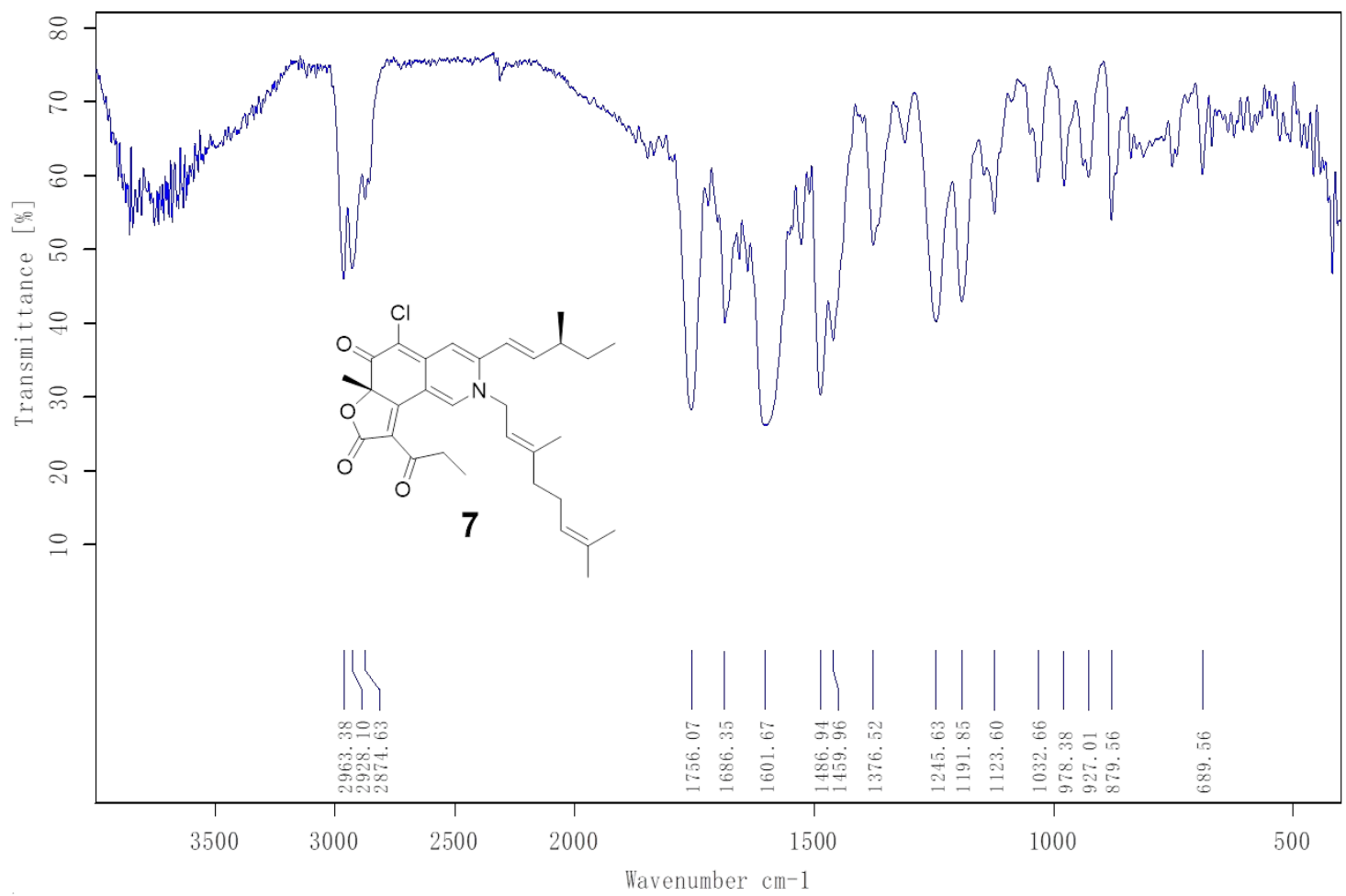

Figure S78. IR spectrum of compound 7 


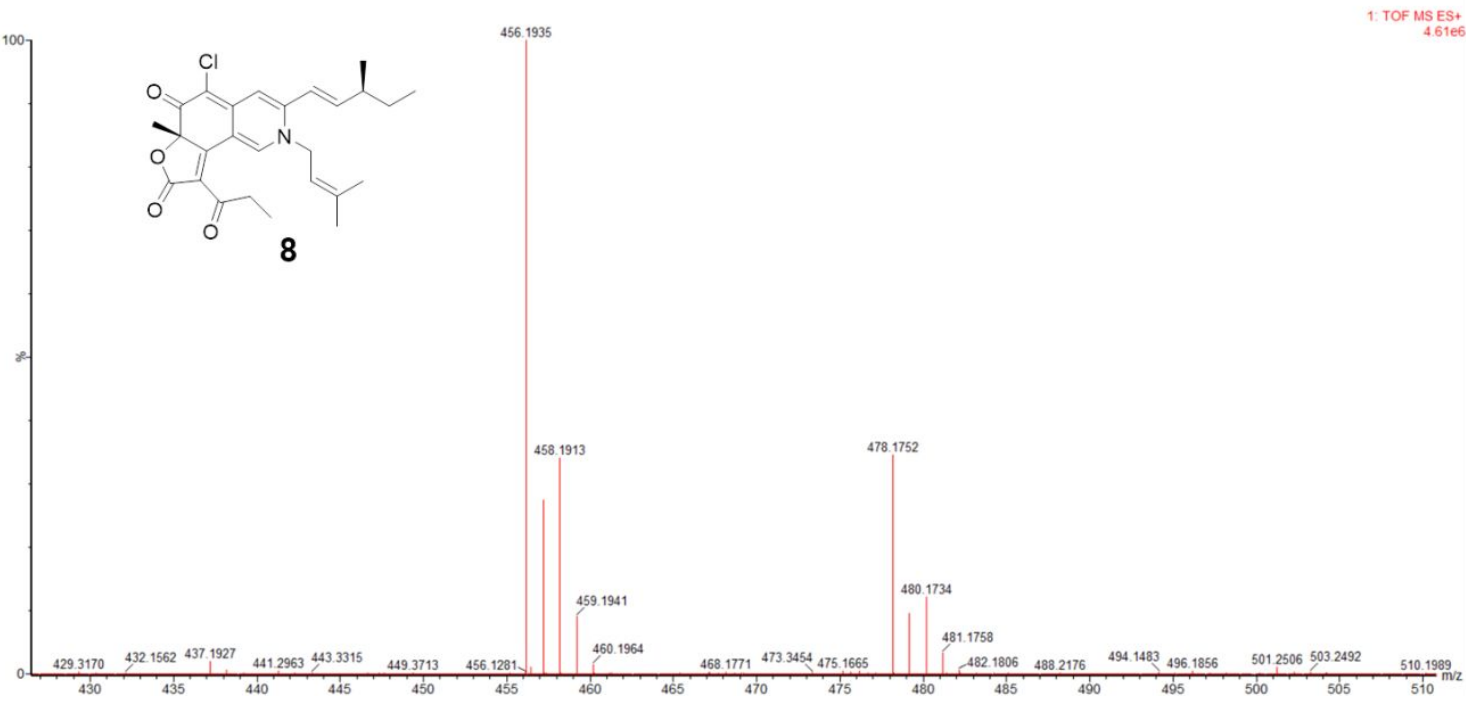

Figure S79. HRESIMS spectrum of compound $\mathbf{8}$

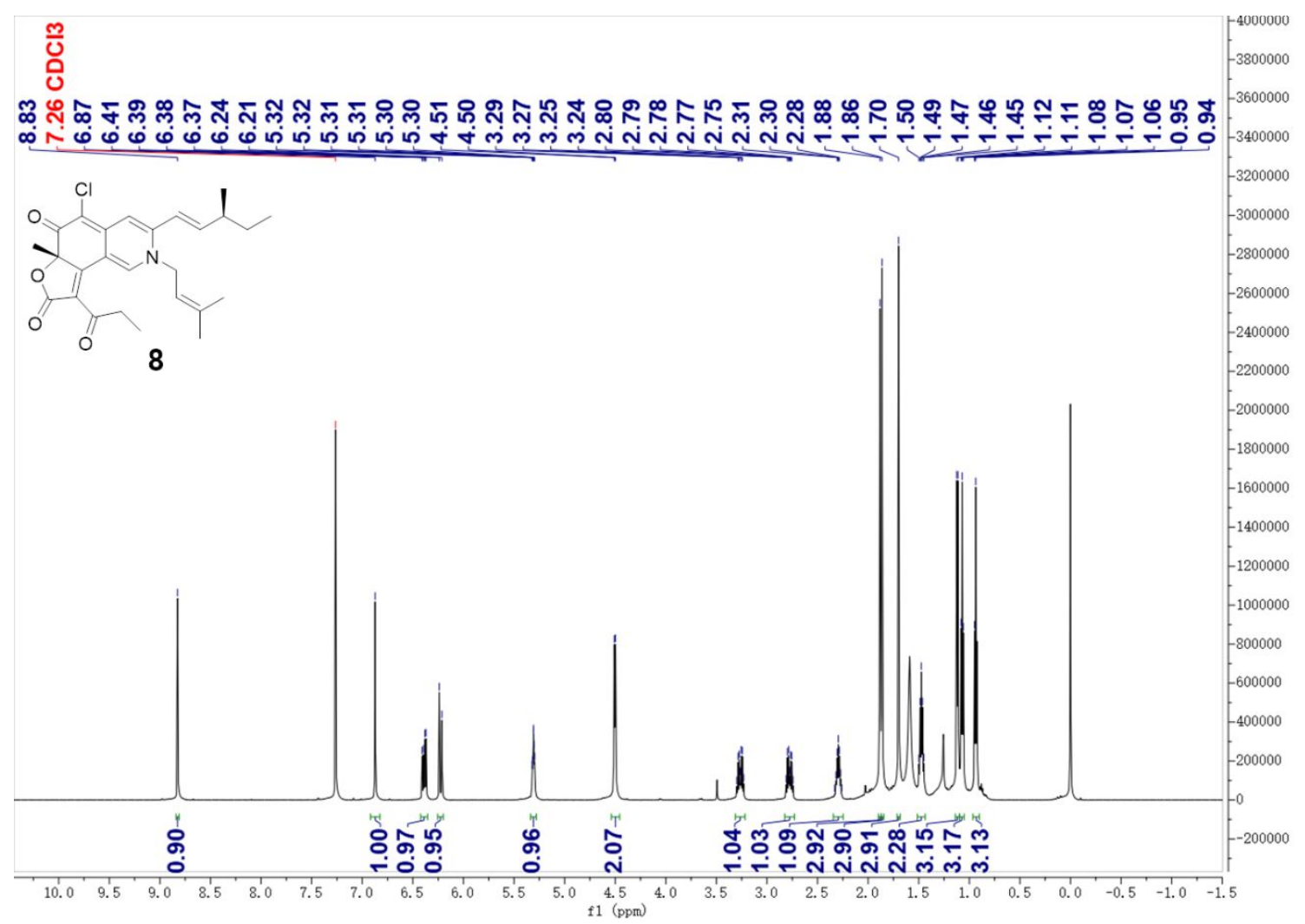

Figure S80. ${ }^{1} \mathrm{H}$ NMR spectrum of compound 8 


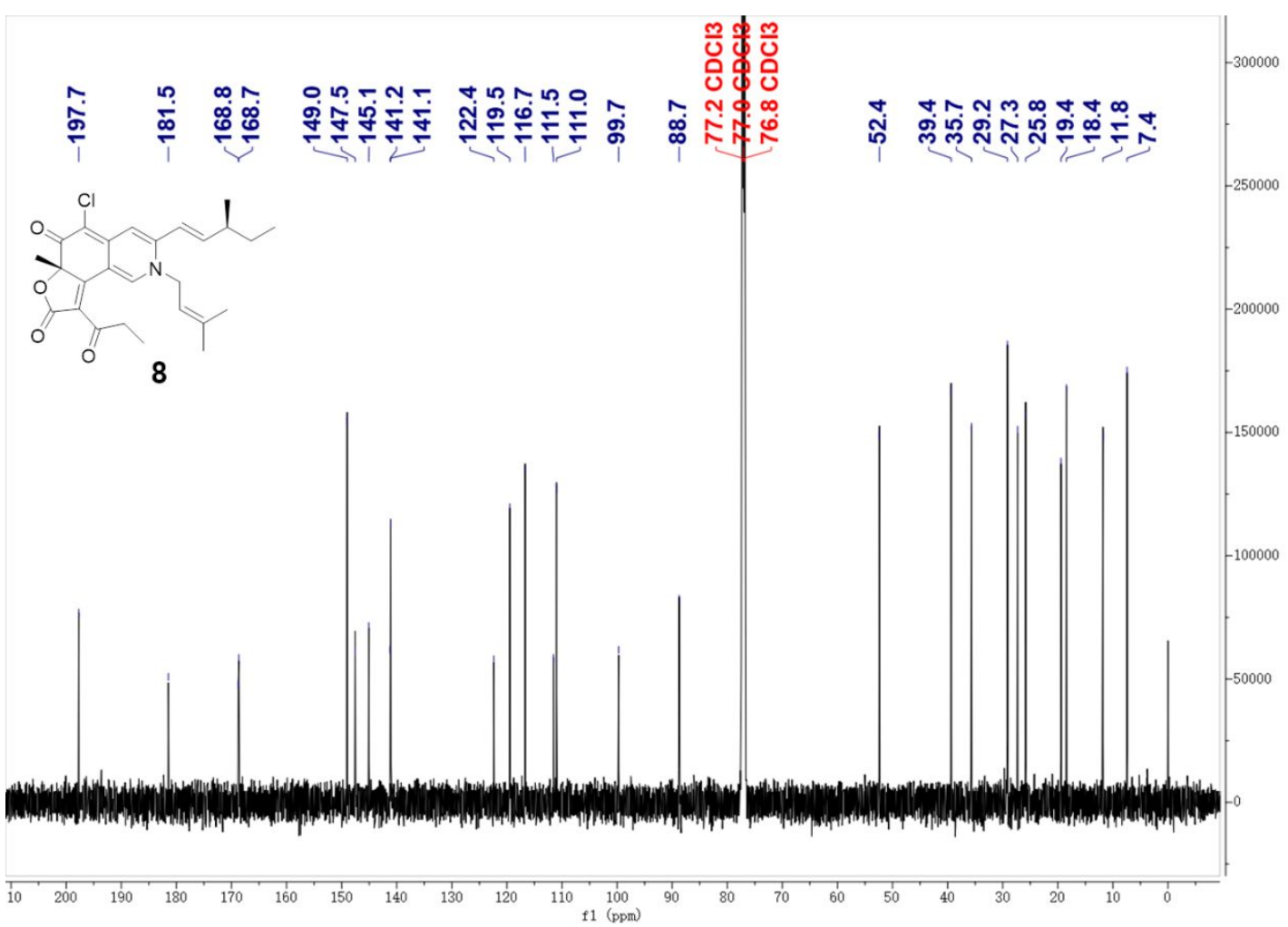

Figure S81. ${ }^{13} \mathrm{C}$ NMR spectrum of compound 8

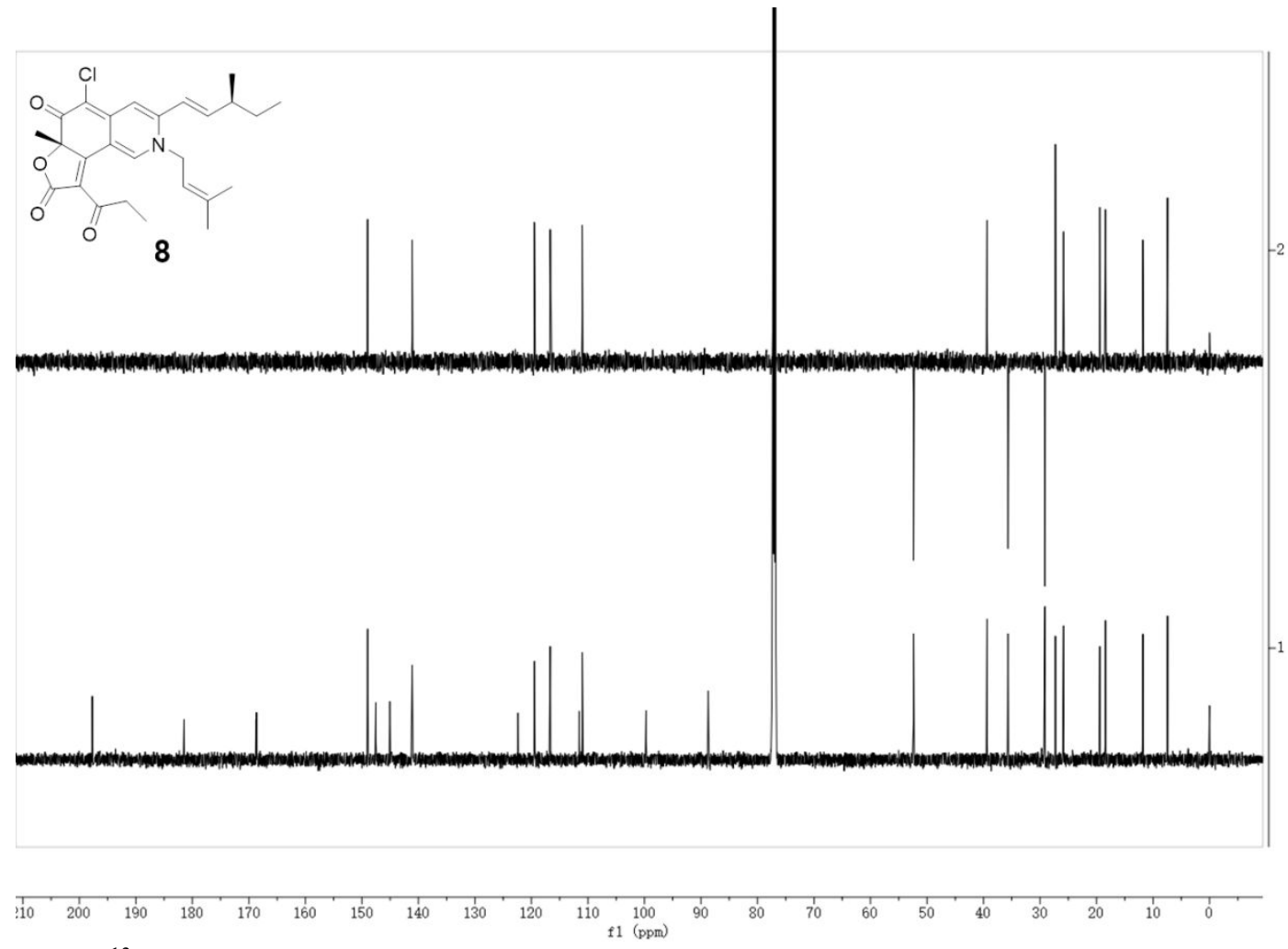

Figure S82. ${ }^{13} \mathrm{C} / \mathrm{DEPT}$ spectrum of compound 8 


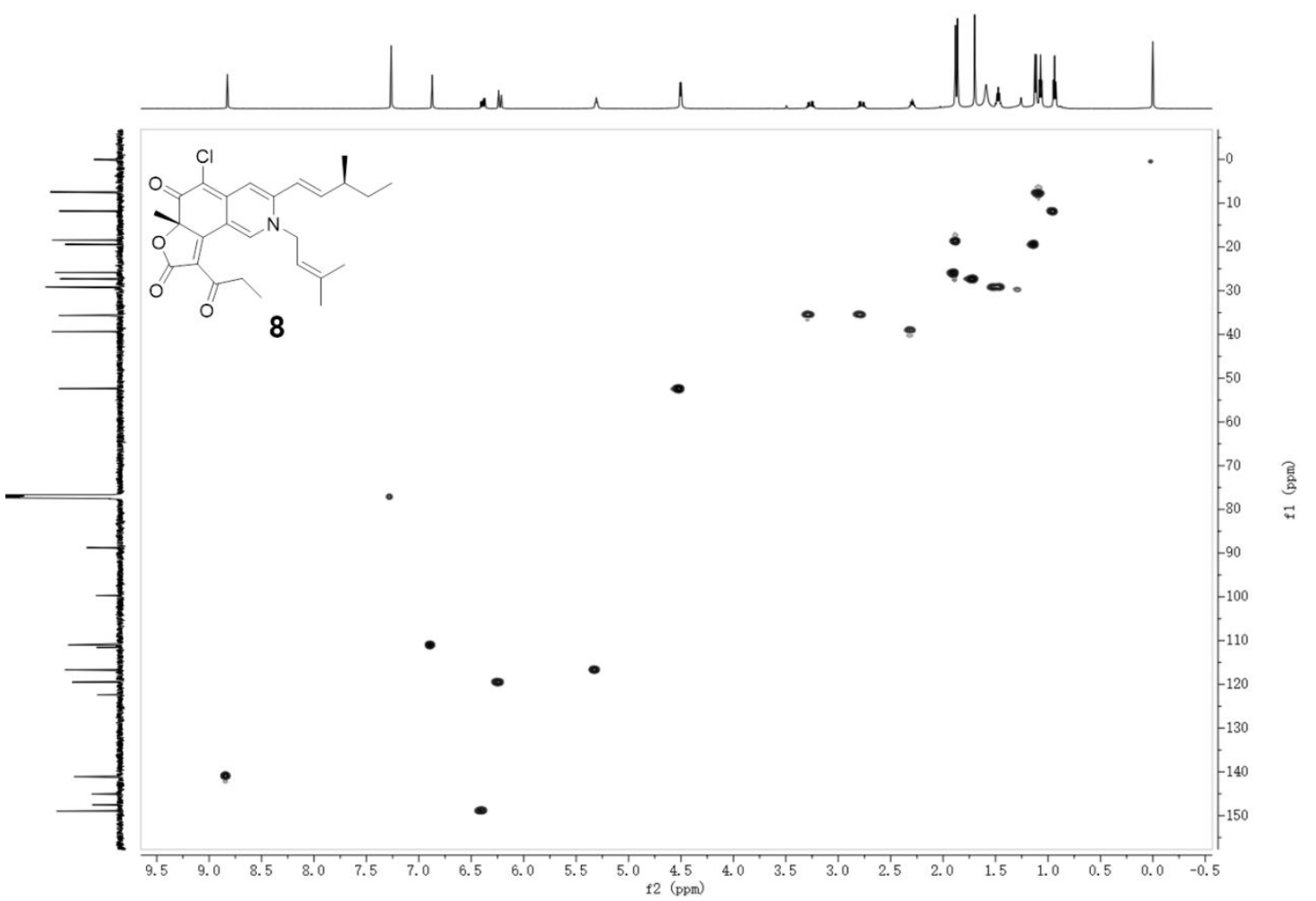

Figure S83. HSQC spectrum of compound 8

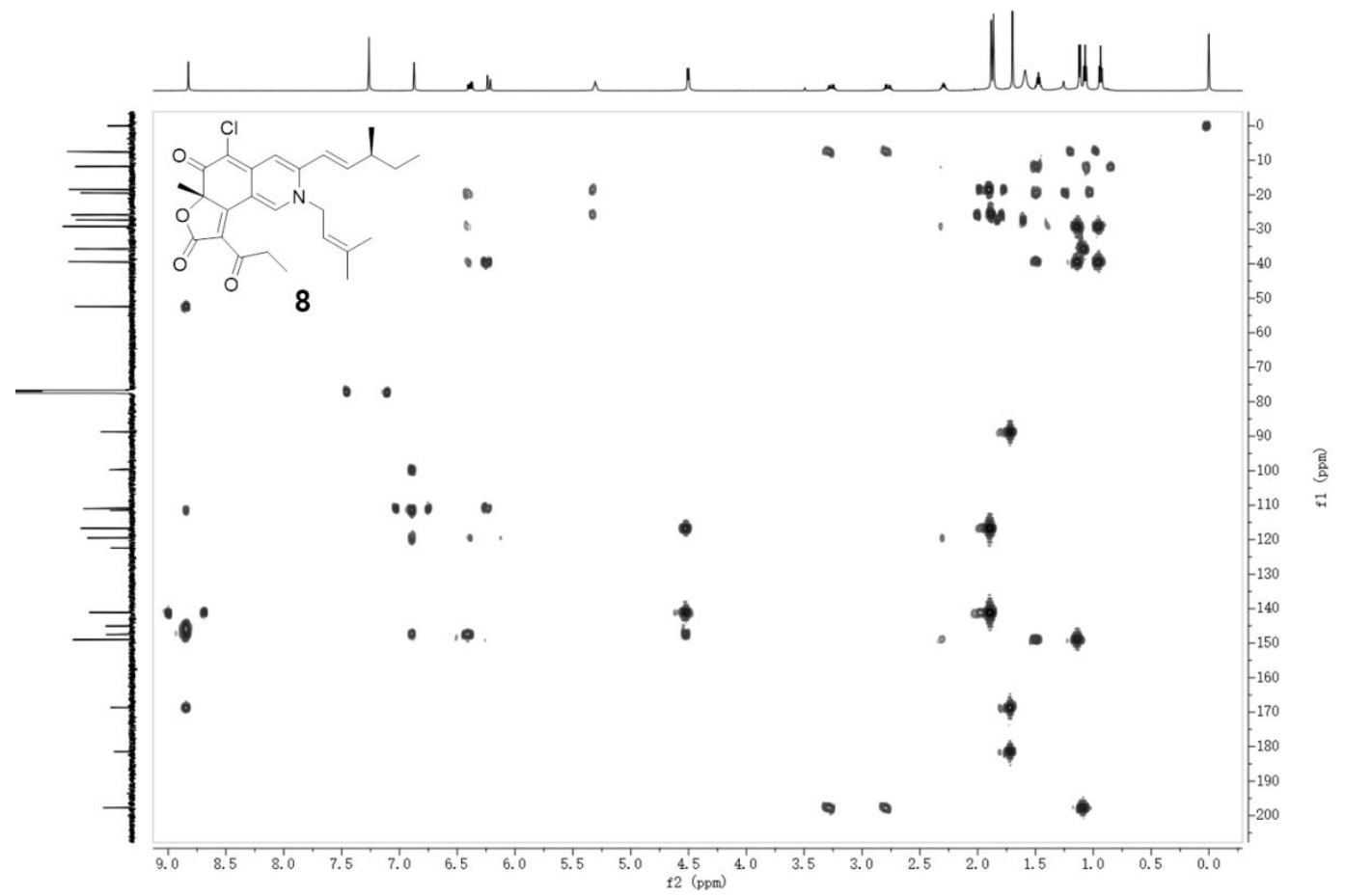

Figure S84. HMBC spectrum of compound 8 


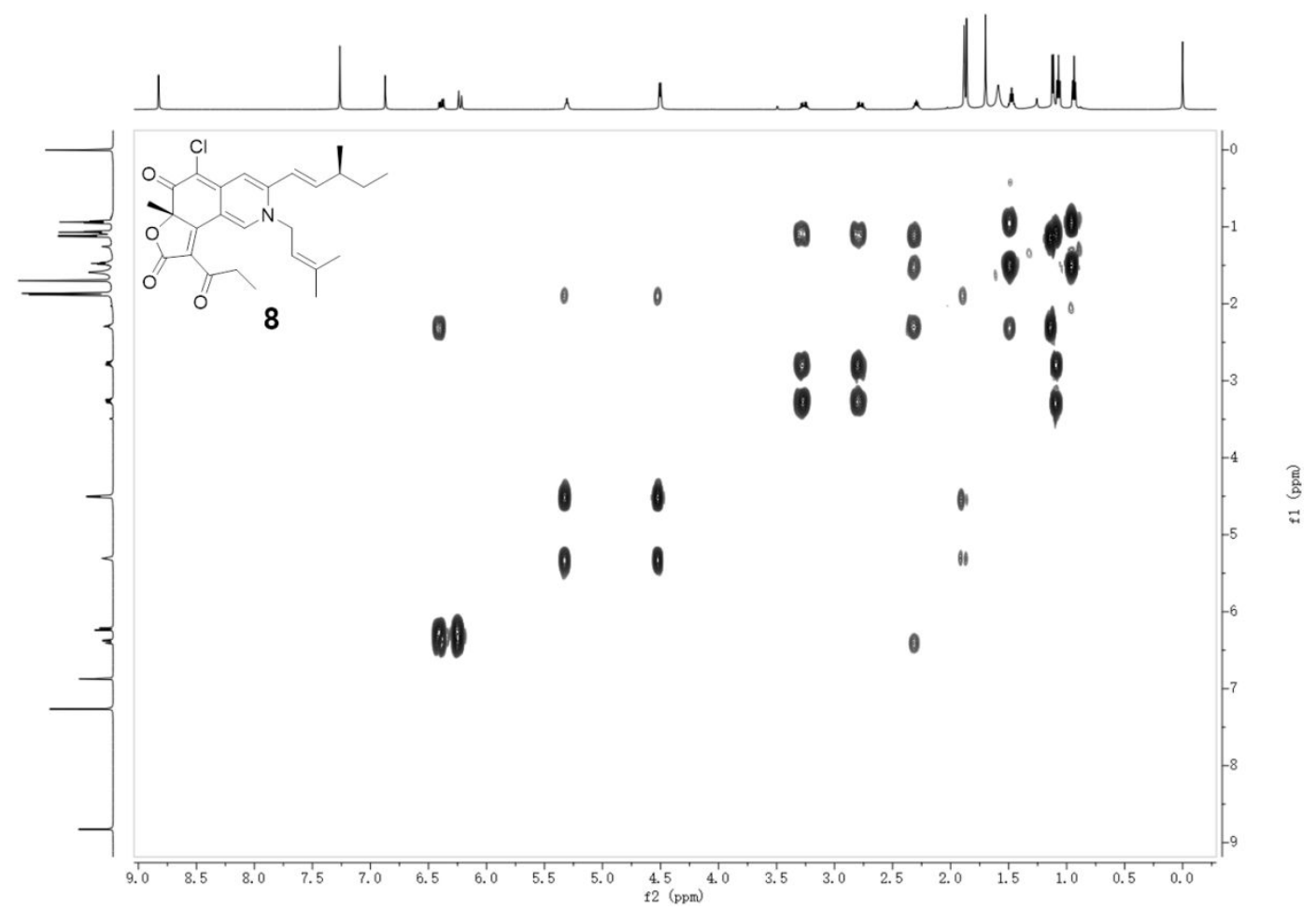

Figure S85. COSY spectrum of compound 8

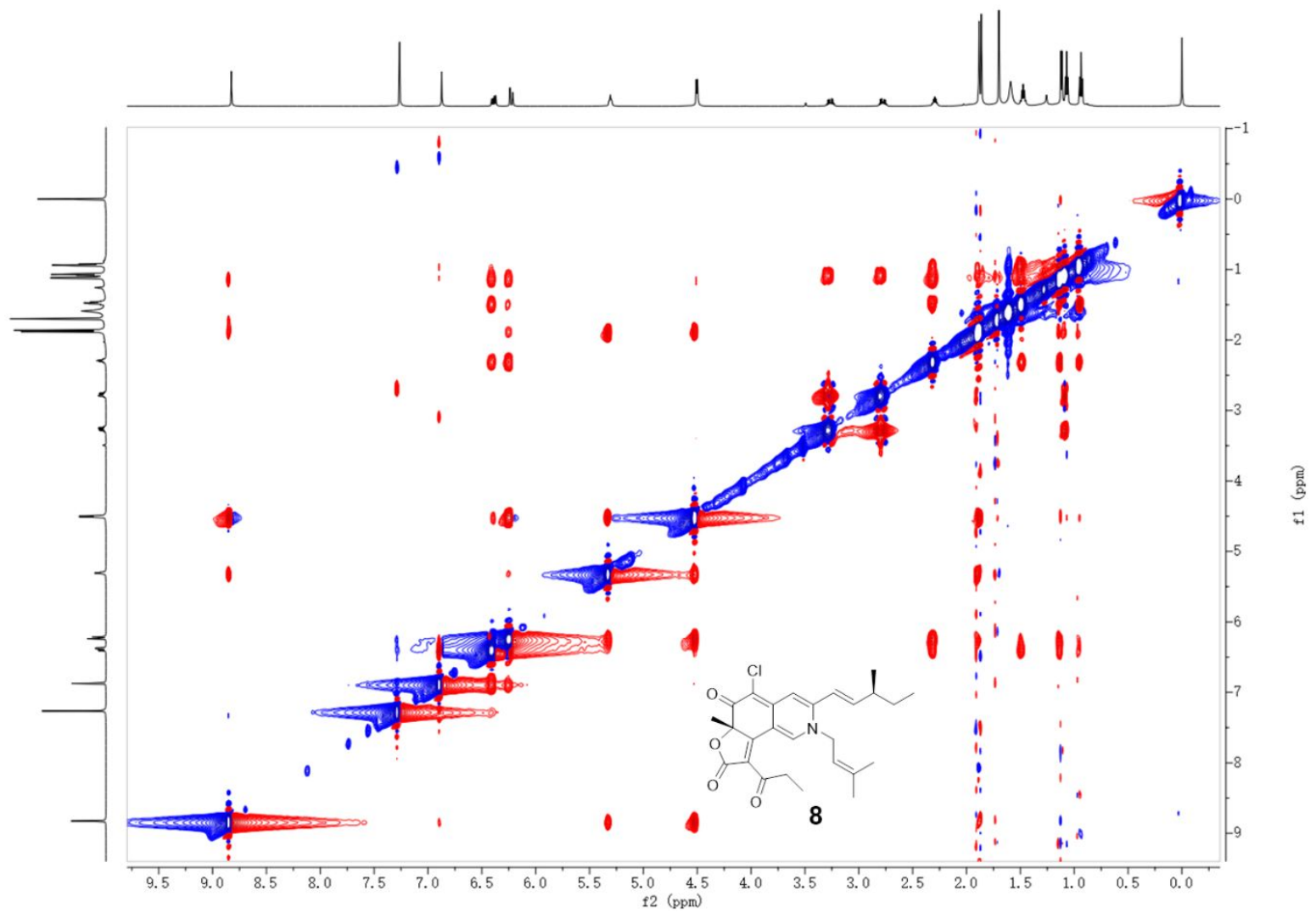

Figure S86. NOESY spectrum of compound $\mathbf{8}$ 


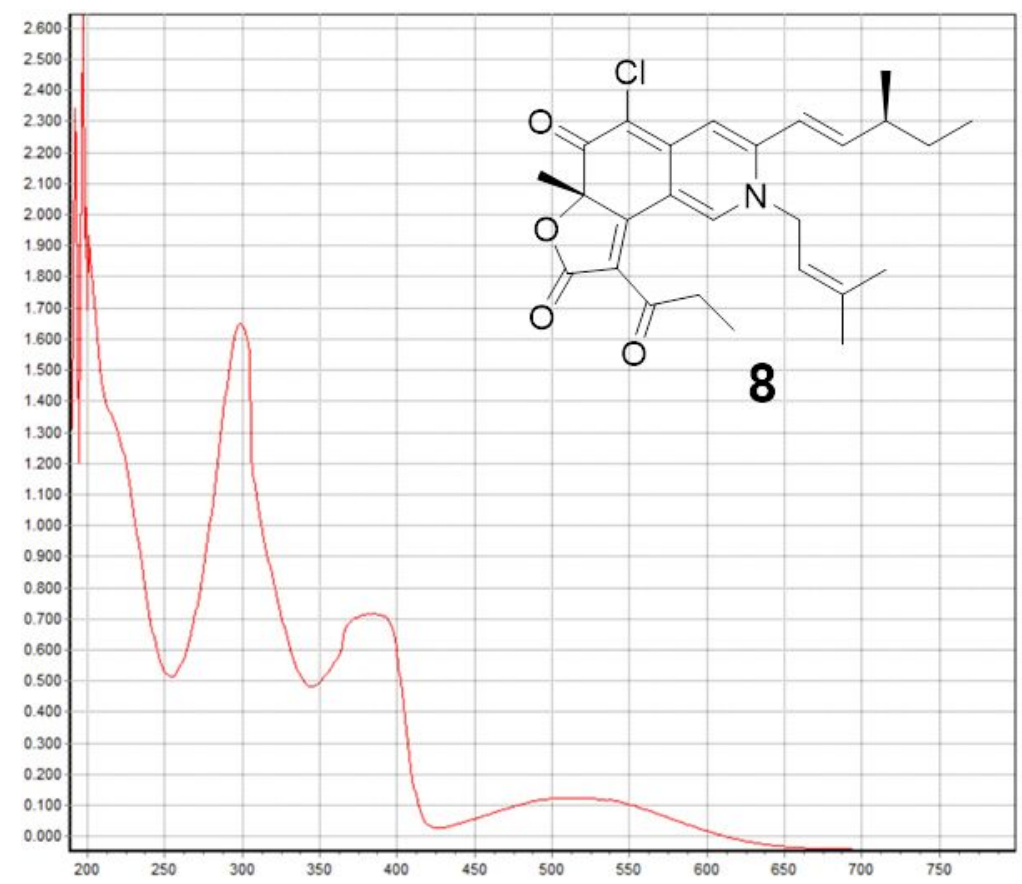

Figure S87. UV spectrum of compound 8

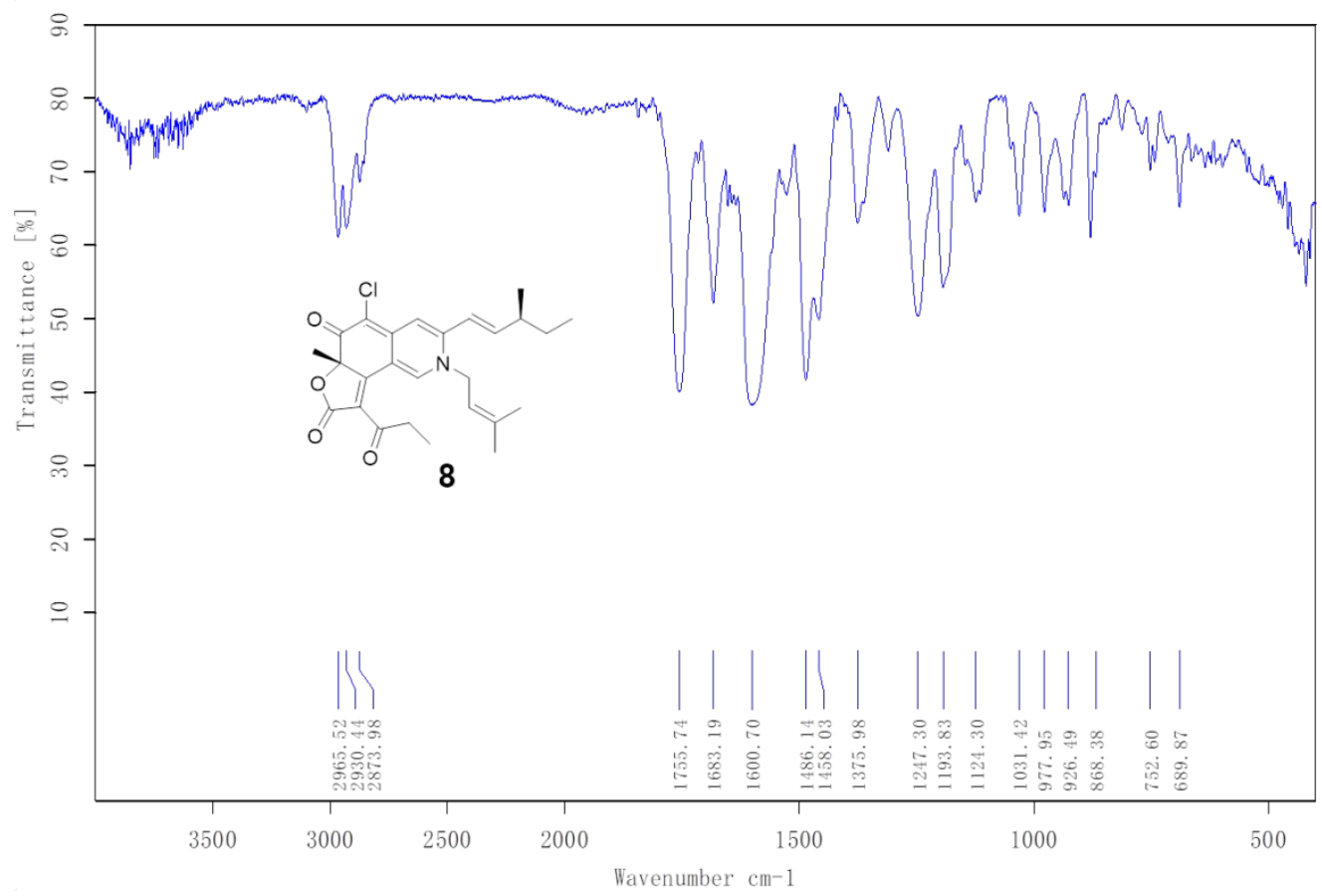

Figure S88. IR spectrum of compound 8 


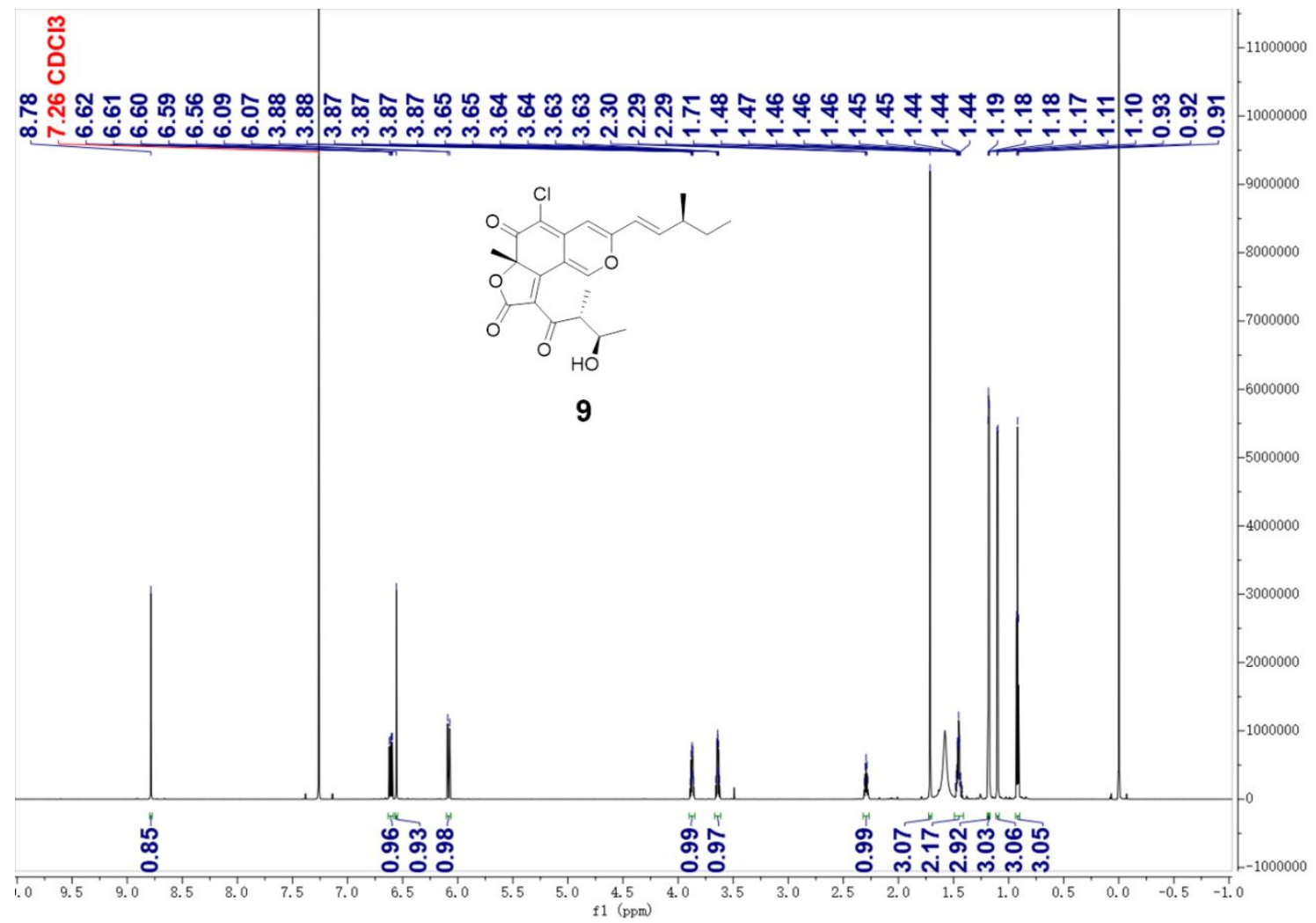

Figure S89. ${ }^{1} \mathrm{H}$ NMR spectrum of compound 9

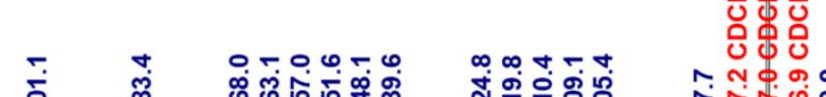

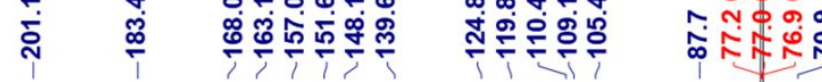

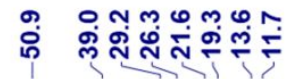

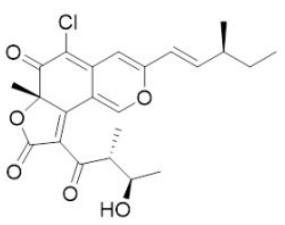

9

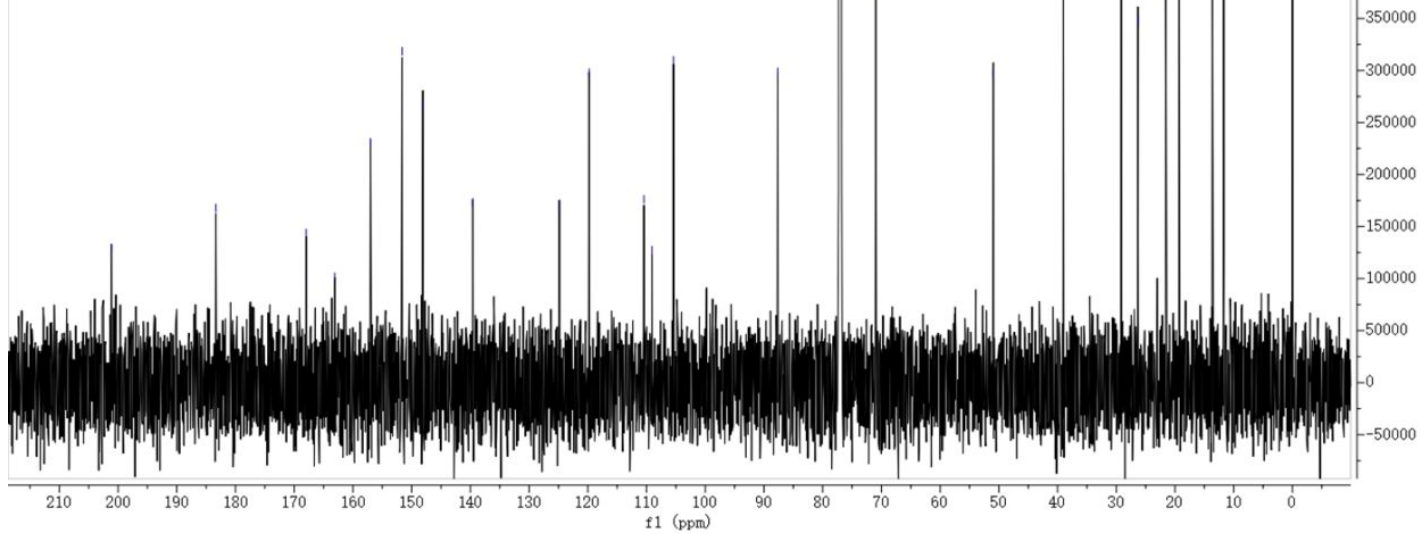

Figure S90. ${ }^{13} \mathrm{C}$ NMR spectrum of compound 9 


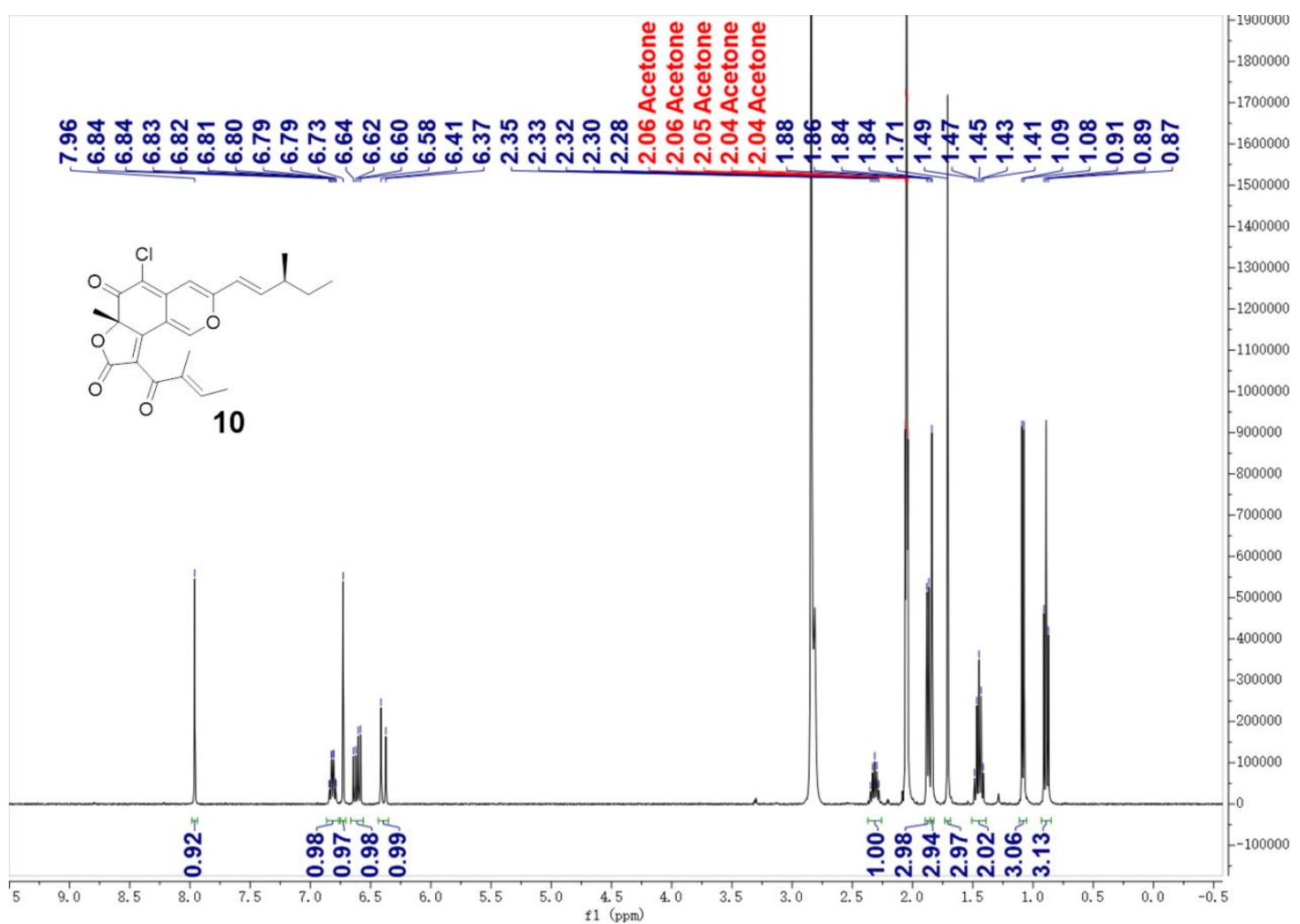

Figure S91. ${ }^{1} \mathrm{H}$ NMR spectrum of compound 10

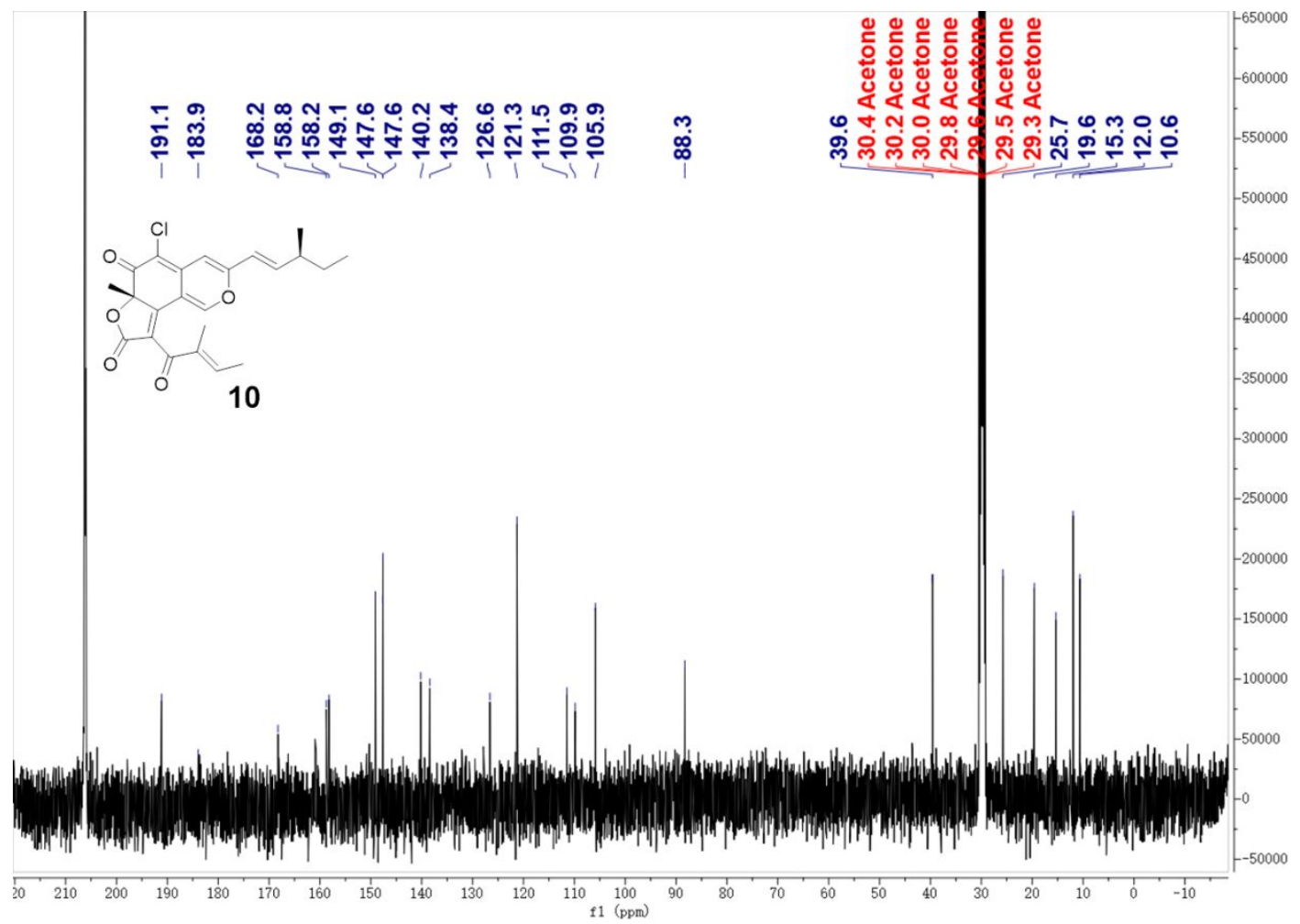

Figure S92. ${ }^{13} \mathrm{C}$ NMR spectrum of compound $\mathbf{1 0}$ 
Table S24. NMR data for compound 9 in chloroform- $d$ (measured in $850 \mathrm{MHz}$ )

\begin{tabular}{|c|c|c|}
\hline \multirow[t]{2}{*}{ No. } & \multicolumn{2}{|c|}{9} \\
\hline & $\delta_{C}$, type & $\delta_{H}(J$ in $\mathrm{Hz})$ \\
\hline 1 & 151.6, CH & $8.78, \mathrm{~s}$ \\
\hline \multicolumn{3}{|l|}{2} \\
\hline 3 & 157.0, C & \\
\hline 4 & $105.4, \mathrm{CH}$ & $6.56, \mathrm{~s}$ \\
\hline $4 \mathrm{a}$ & 139.6, C & \\
\hline 5 & 109.1, C & \\
\hline 6 & 183.4, C & \\
\hline 7 & $87.7, \mathrm{C}$ & \\
\hline 8 & 163.1, C & \\
\hline $8 a$ & $110.4, \mathrm{C}$ & \\
\hline 9 & $119.8, \mathrm{CH}$ & $6.08, \mathrm{~d}(15.6)$ \\
\hline 10 & 148.1, CH & $6.61, \mathrm{dd}(15.8,8.0)$ \\
\hline 11 & $39.0, \mathrm{CH}$ & $2.29, \mathrm{~m}$ \\
\hline 12 & $29.2, \mathrm{CH}_{2}$ & $1.45, \mathrm{~m}$ \\
\hline 13 & $11.7, \mathrm{CH}_{3}$ & $0.92, \mathrm{t}(7.4)$ \\
\hline 14 & $19.3, \mathrm{CH}_{3}$ & $1.10, \mathrm{~d}(6.8)$ \\
\hline 15 & $26.3, \mathrm{CH}_{3}$ & $1.71, \mathrm{~s}$ \\
\hline $1^{\prime}$ & 168.0, C & \\
\hline $2^{\prime}$ & $124.8, \mathrm{C}$ & \\
\hline $3^{\prime}$ & 201.1, C & \\
\hline $4^{\prime}$ & $50.9, \mathrm{CH}$ & $3.64, \mathrm{qd}(6.8,8.0)$ \\
\hline $5^{\prime}$ & $70.9, \mathrm{CH}$ & $3.87, \mathrm{qd}(6.4,8.0)$ \\
\hline $6^{\prime}$ & 21.6, $\mathrm{CH}_{3}$ & $1.18, \mathrm{~d}(6.4)$ \\
\hline $7^{\prime}$ & 13.6, $\mathrm{CH}_{3}$ & $1.17, \mathrm{~d}(6.8)$ \\
\hline
\end{tabular}




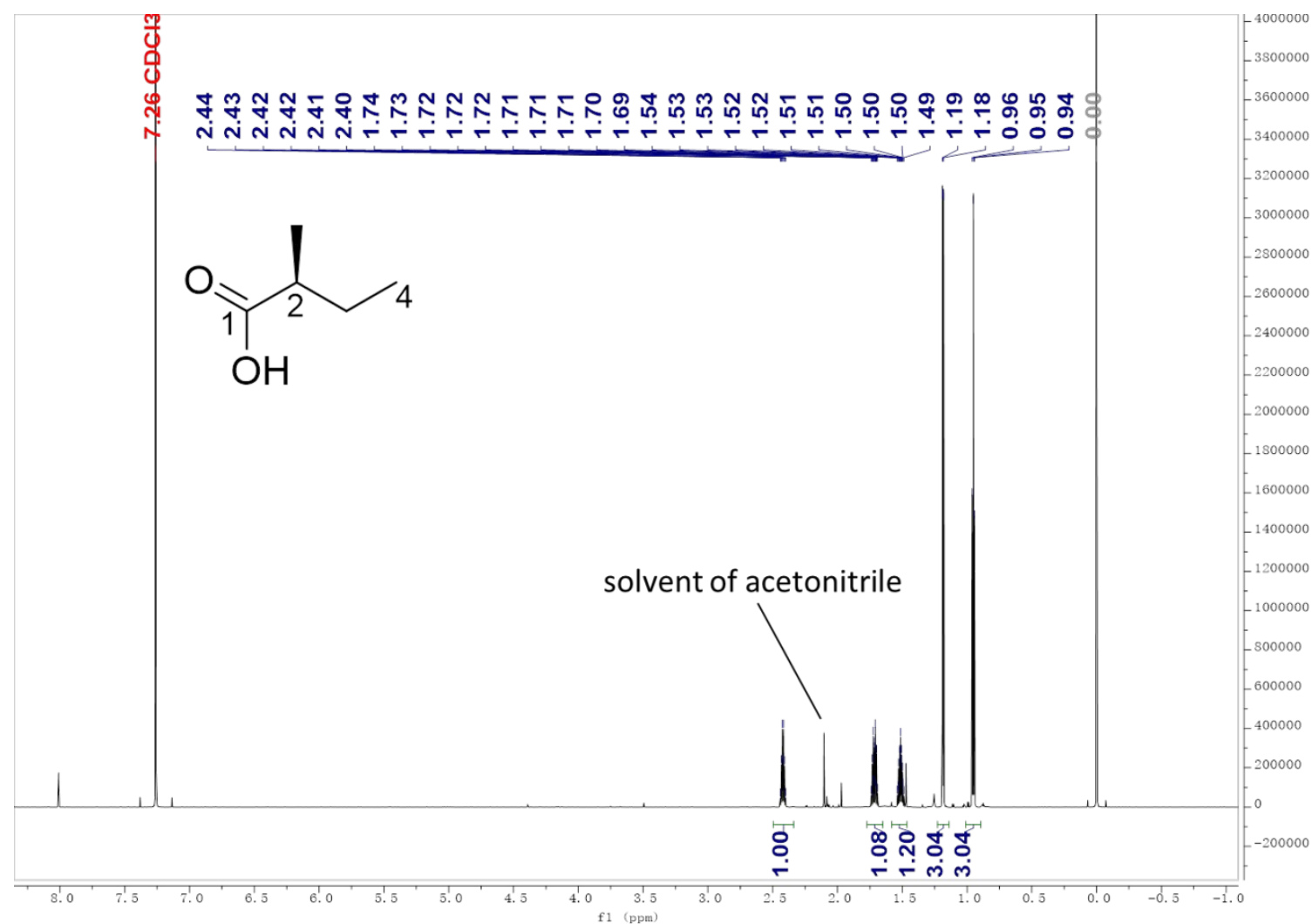

Figure S93. ${ }^{1} \mathrm{H}$ NMR spectrum of 2-methylbutyric acid

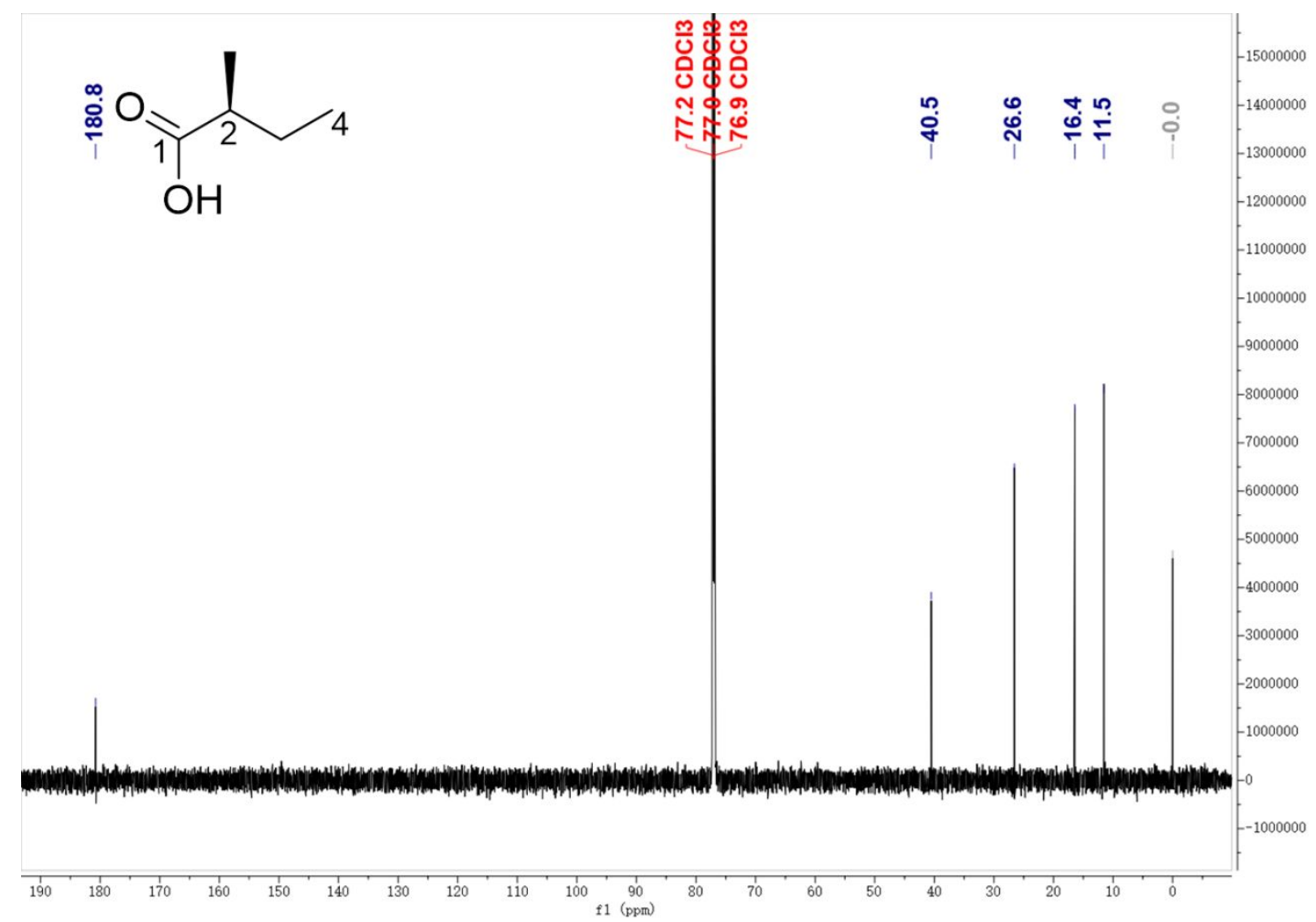

Figure S94. ${ }^{13} \mathrm{C}$ NMR spectrum of 2-methylbutyric acid 


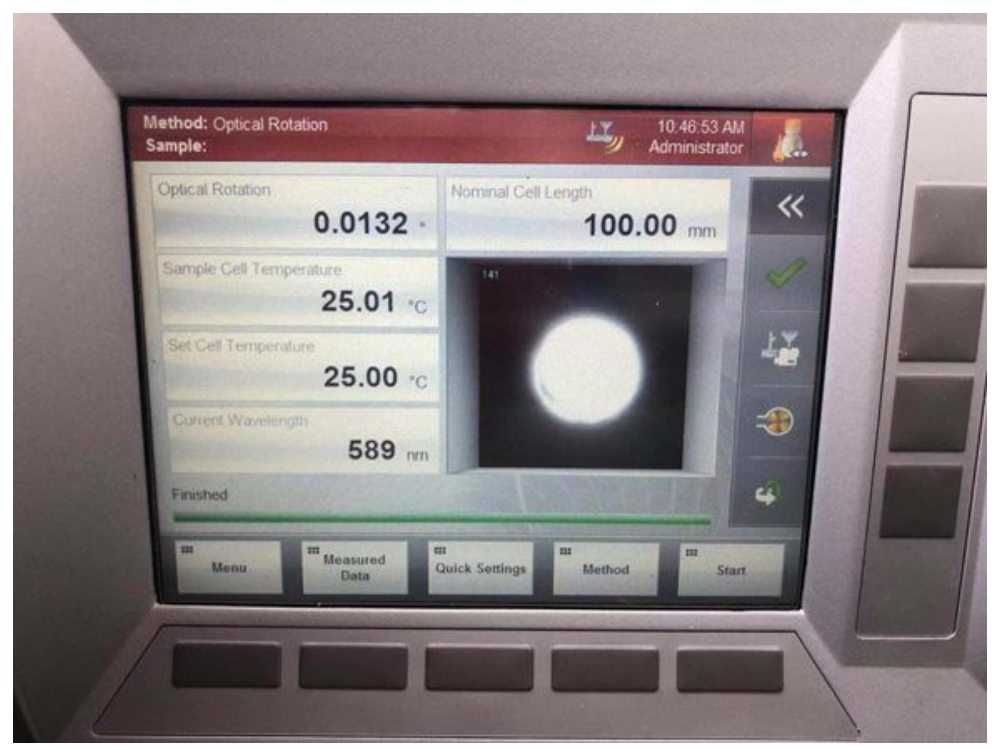

Figure S95. Optical rotation of $(S)-(+)-2$-methylbutyric acid at the concentration of $1 \mathrm{mg} / \mathrm{ml}$ in chloroform.

Table S25. NMR data for 2-methylbutyric acid in chloroform- $d$ (measured in $850 \mathrm{MHz}$ )

\begin{tabular}{ccc}
\hline No. & $\delta_{C}$, type & $\delta_{H}(J$ in $\mathrm{Hz})$ \\
\hline 1 & $180.8, \mathrm{C}$ & - \\
2 & $40.5, \mathrm{CH}$ & $2.42, \mathrm{~m}$ \\
3 & $26.6, \mathrm{CH}_{2}$ & $1,51,1.72, \mathrm{~m}$ \\
4 & $11.5, \mathrm{CH}_{3}$ & $0.97, \mathrm{t}(7.5)$ \\
$2-\mathrm{CH}_{3}$ & $16.4, \mathrm{CH}_{3}$ & $1.19, \mathrm{~d}(7.0)$ \\
\hline
\end{tabular}

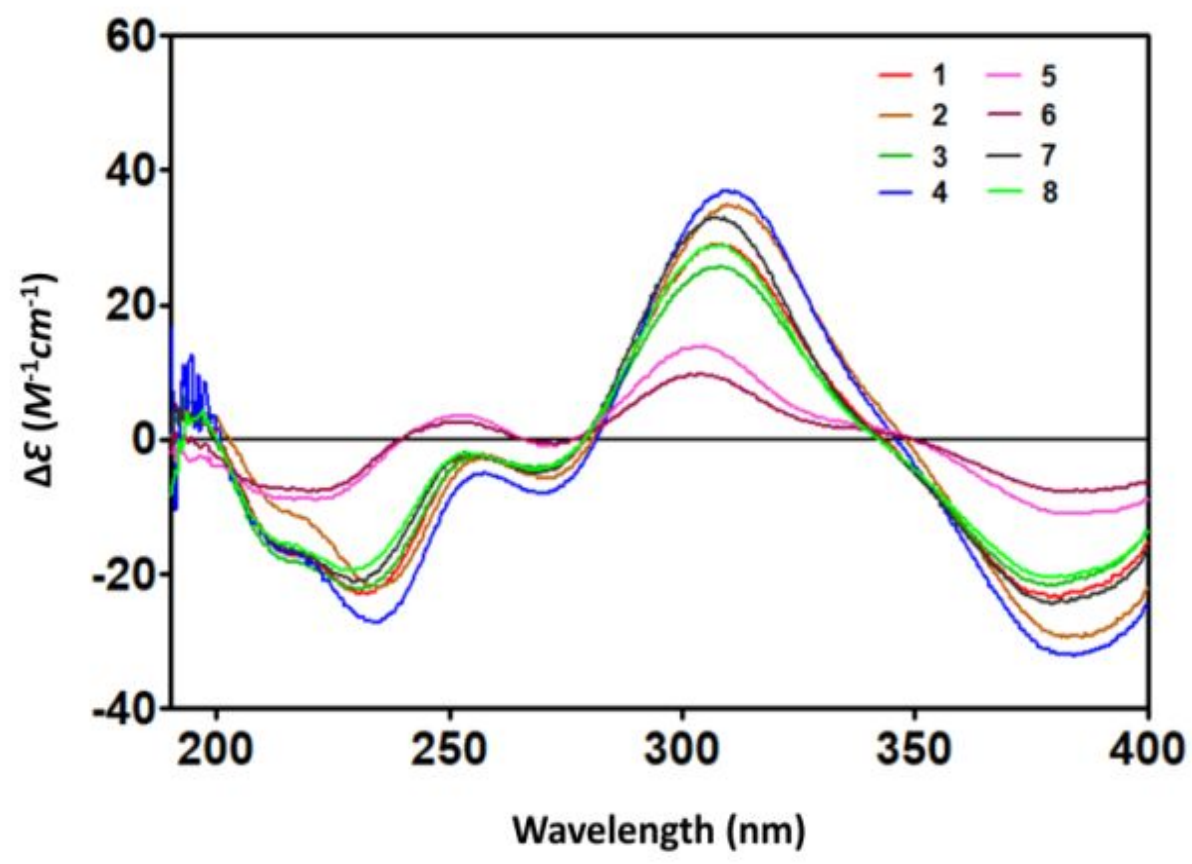


Figure S96. CD spectra of compounds 1-8

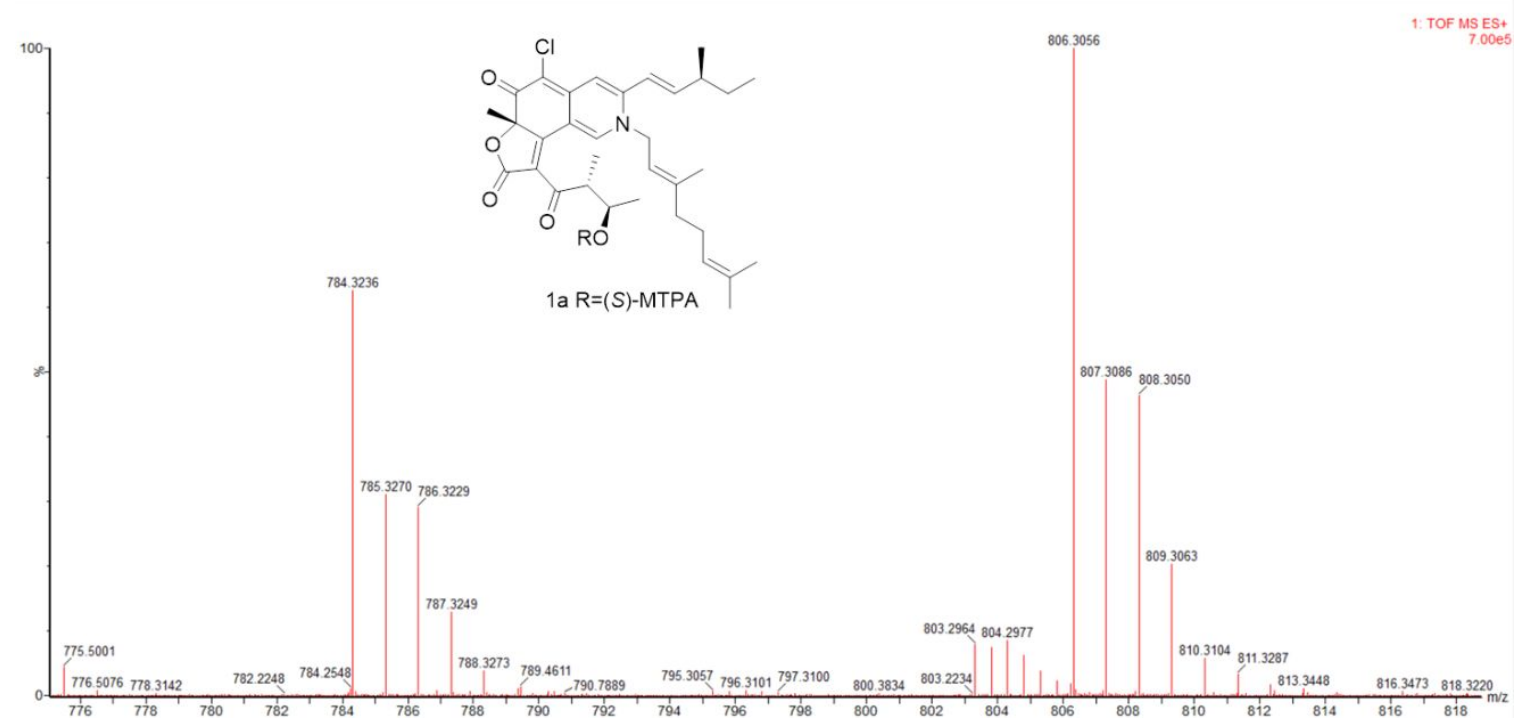

Figure S97. HRESIMS spectrum of (S)-MTPA ester of 1 (1a)

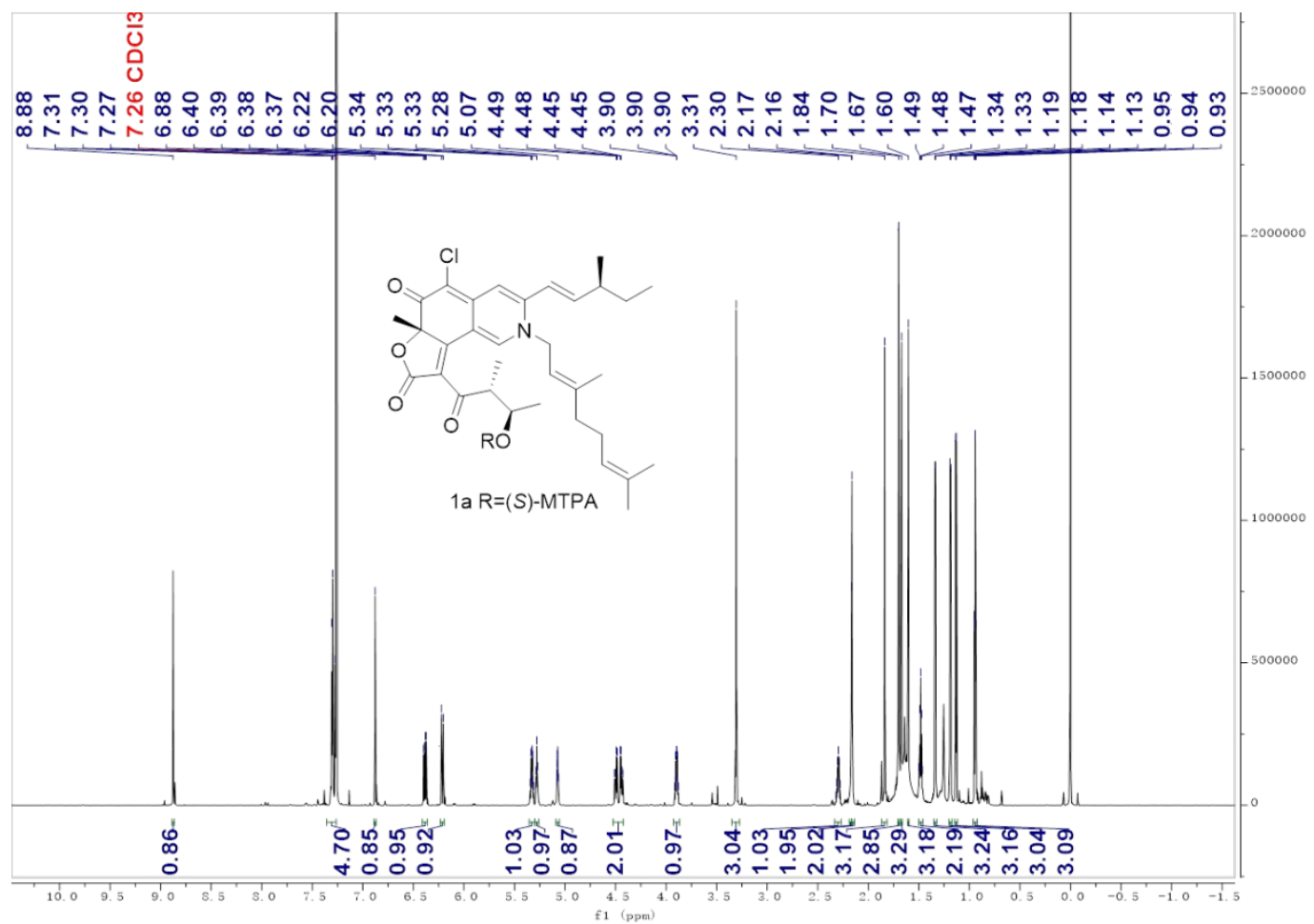

Figure S98. ${ }^{1} \mathrm{H}$ NMR spectrum of (S)-MTPA ester of 1 (1a) 


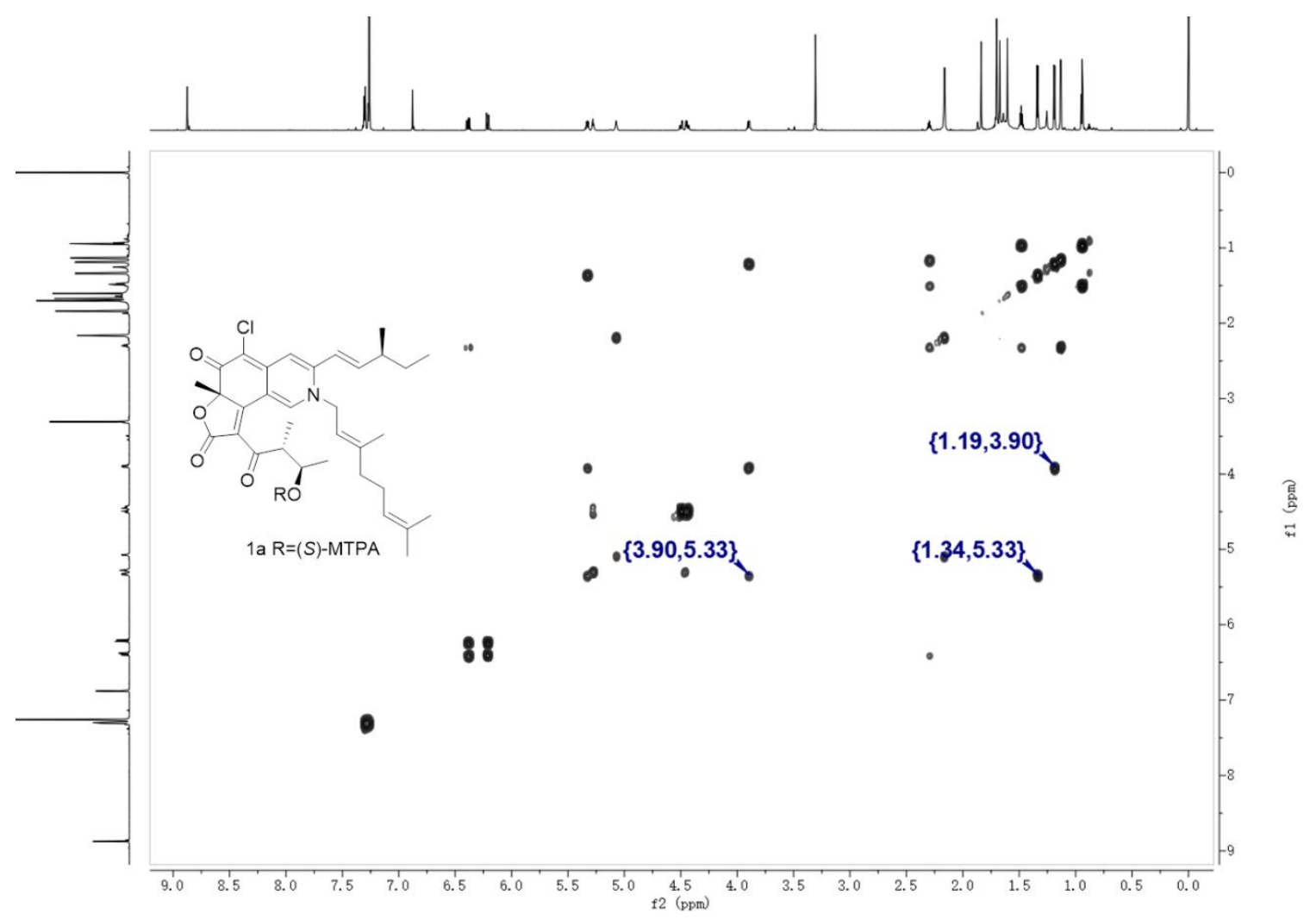

Figure S99. COSY spectrum of (S)-MTPA ester of 1 (1a)

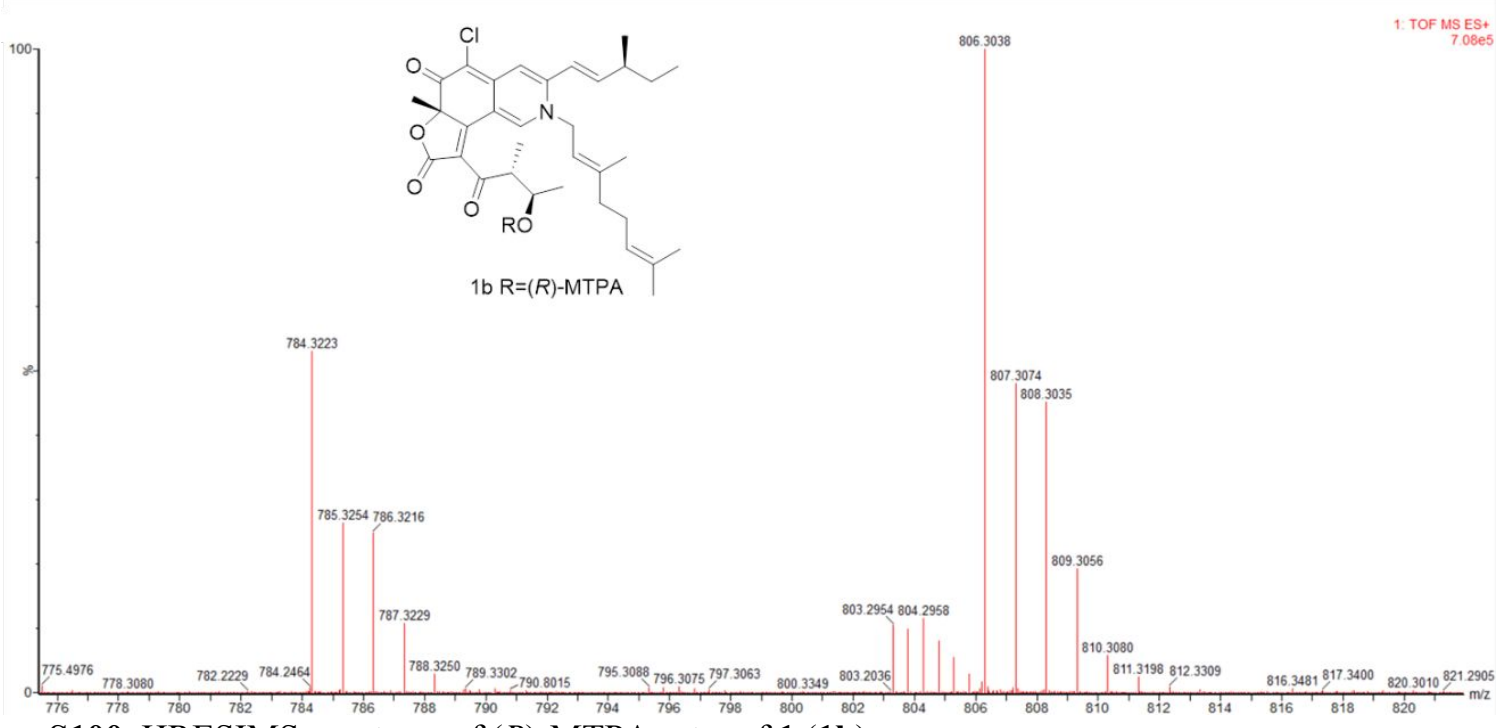

Figure S100. HRESIMS spectrum of $(R)$-MTPA ester of $\mathbf{1}$ (1b) 


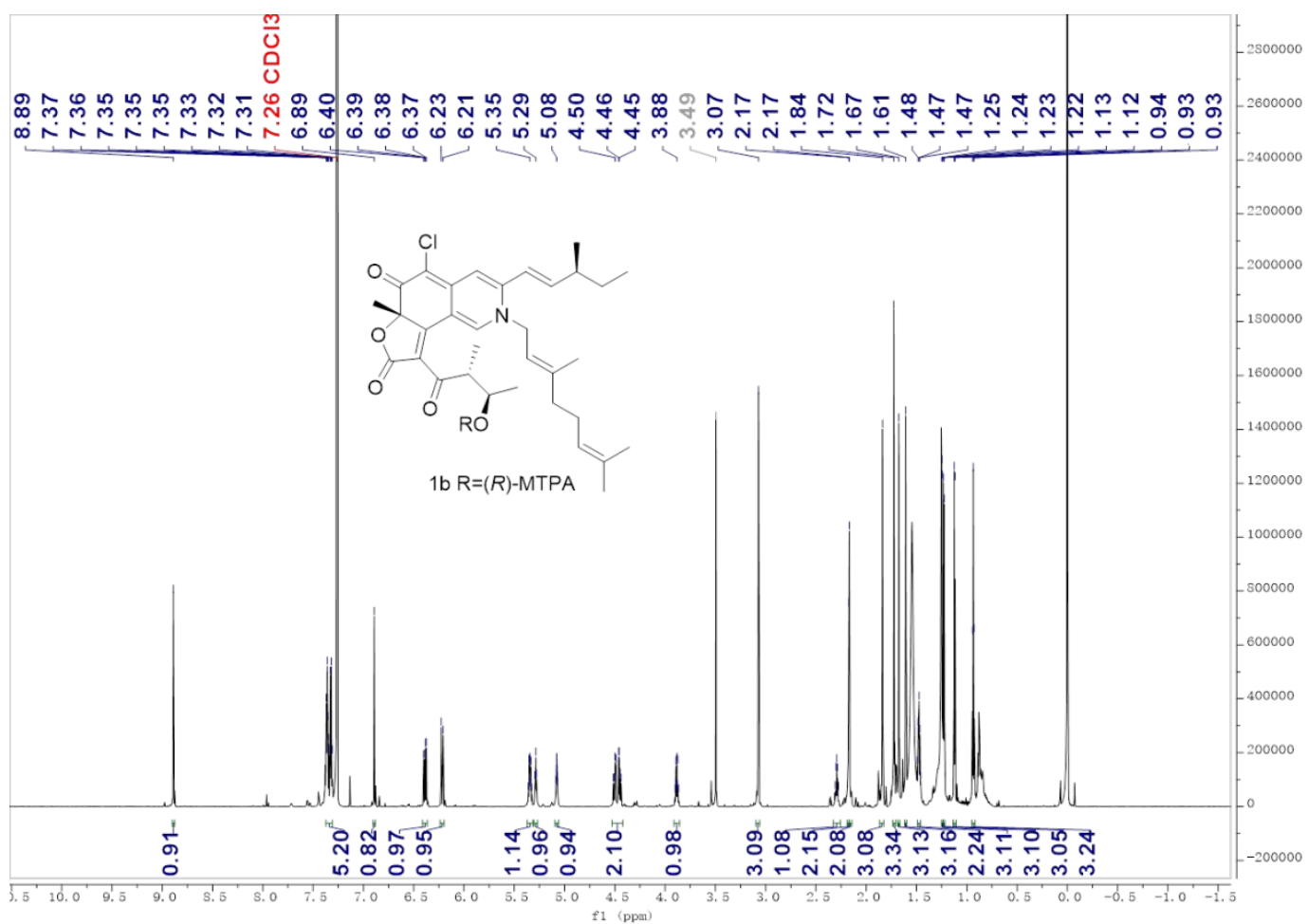

Figure S101. ${ }^{1} \mathrm{H}$ NMR spectrum of $(R)$-MTPA ester of 1 (1b)

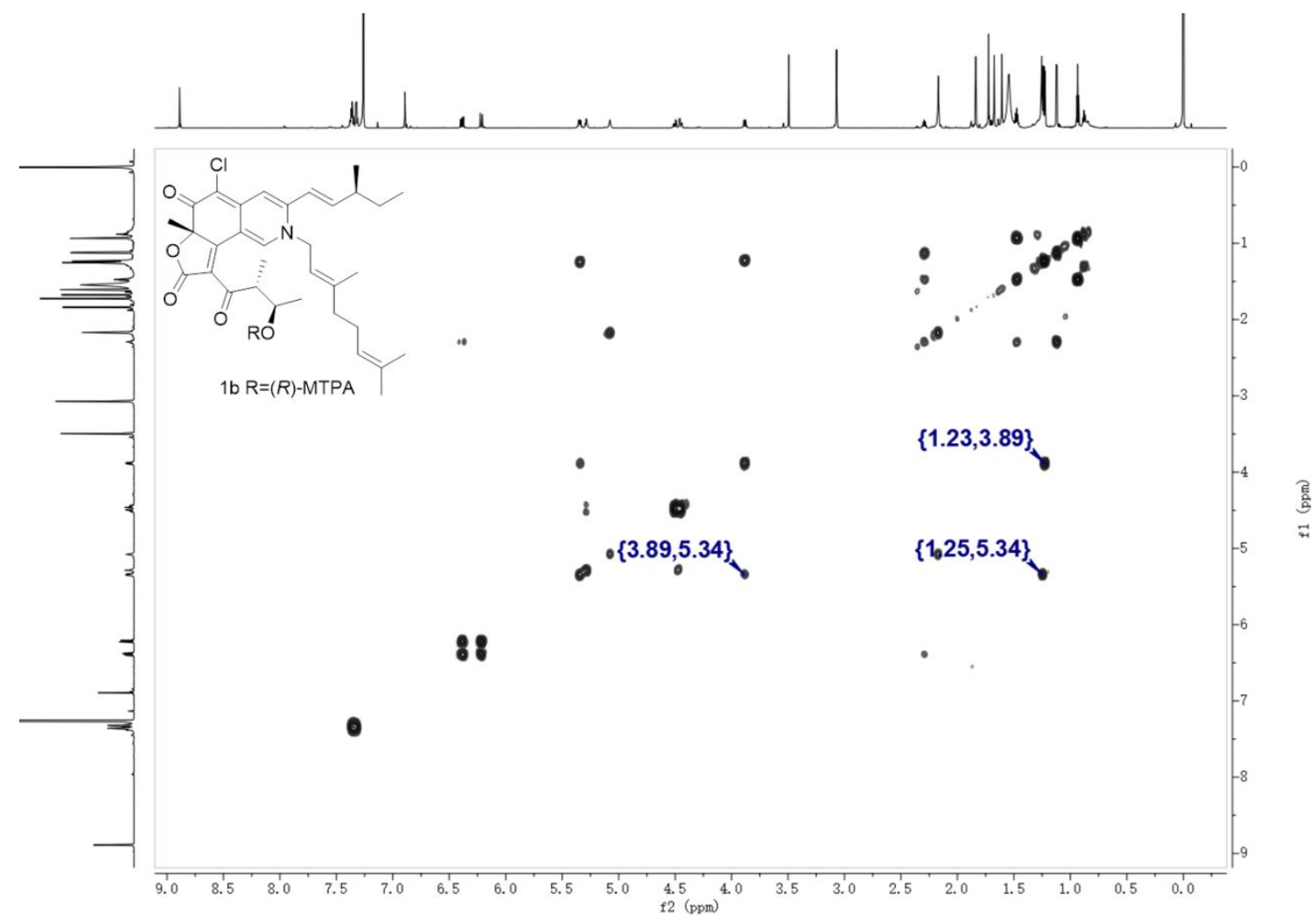

Figure S102. COSY spectrum of $(R)$-MTPA ester of 1 (1b) 


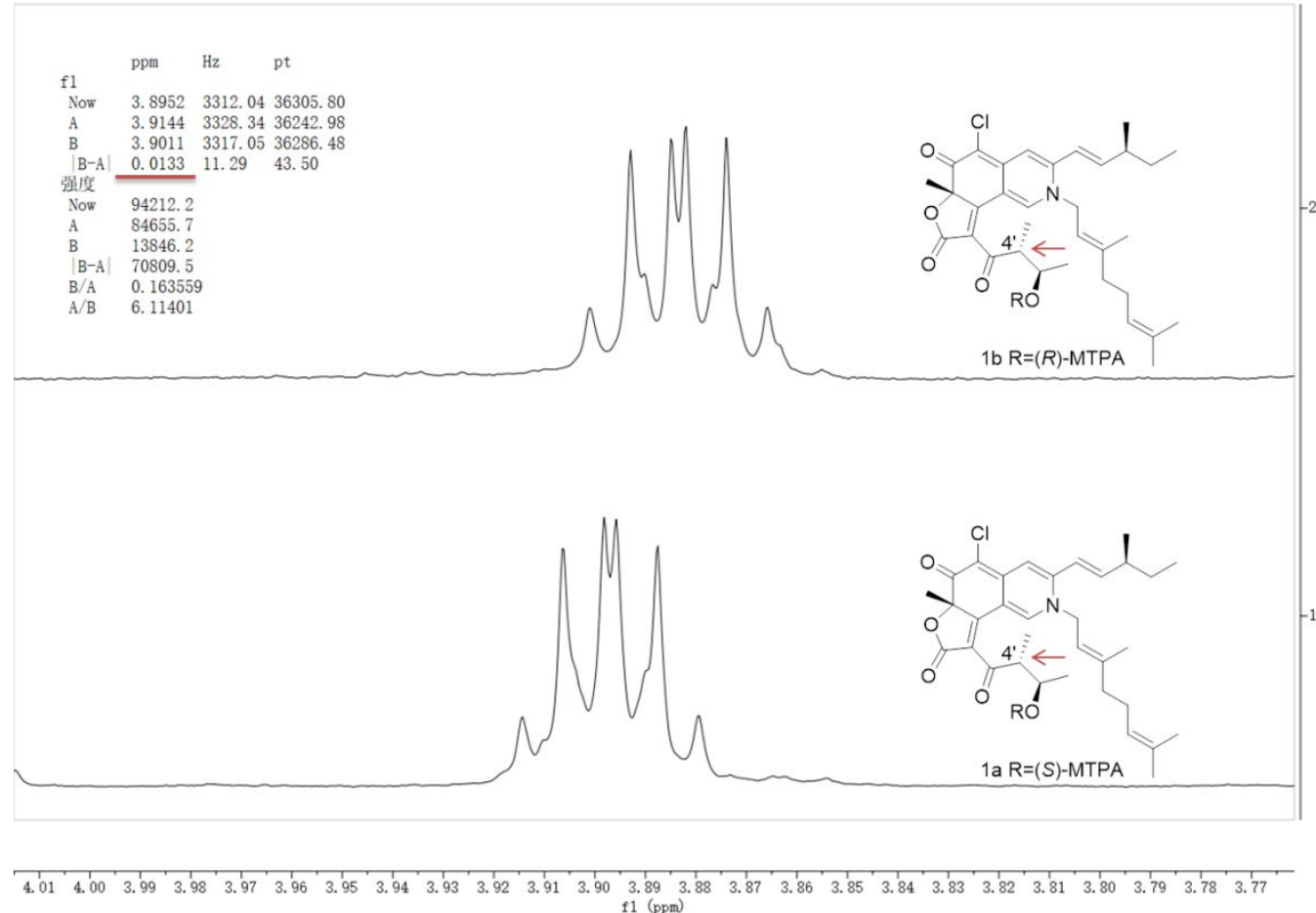

Figure S103. MTPA ester analysis (value was $\Delta \delta_{H}=\delta_{S}-\delta_{R}$ ) of compound 1 at C-4'.

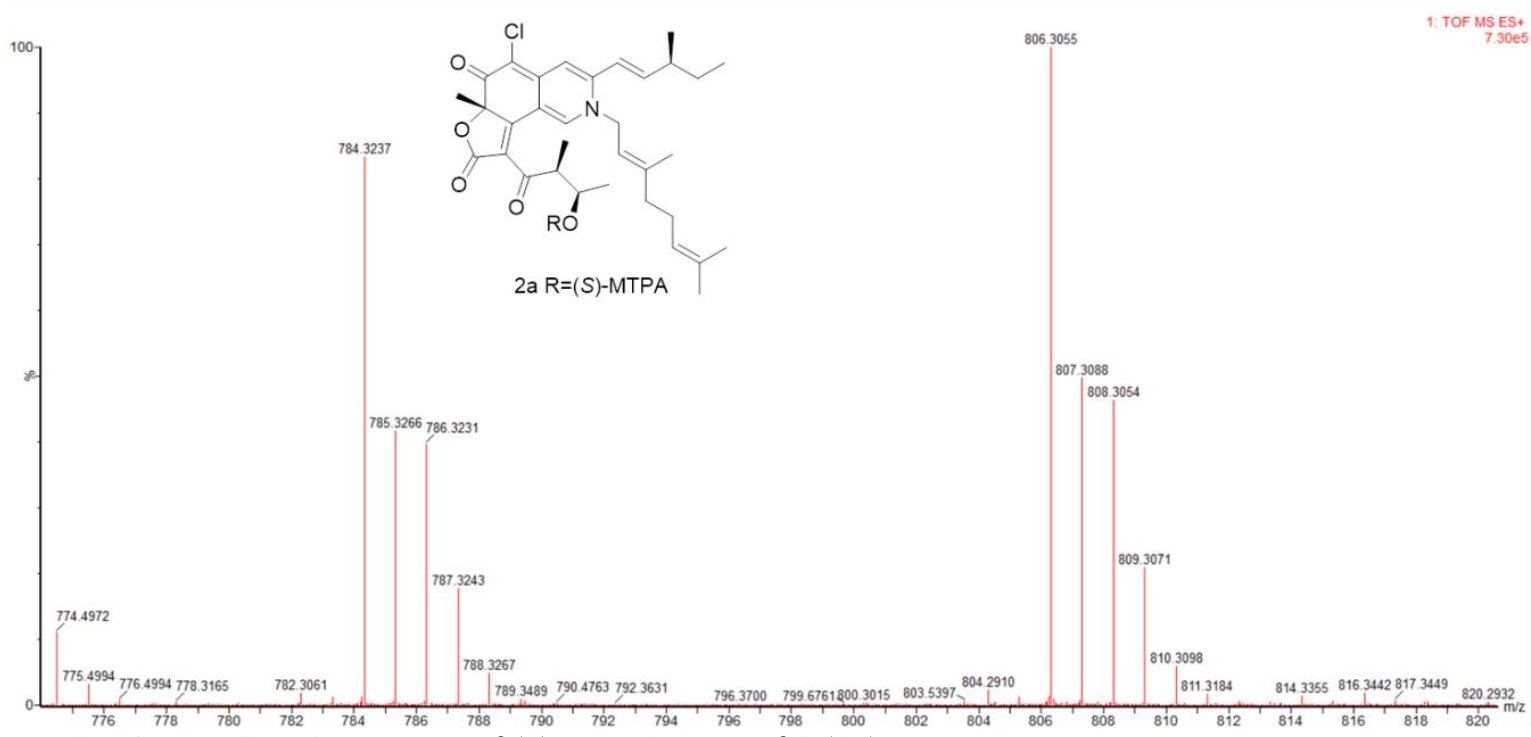

Figure S104. HRESIMS spectrum of (S)-MTPA ester of 2 (2a) 


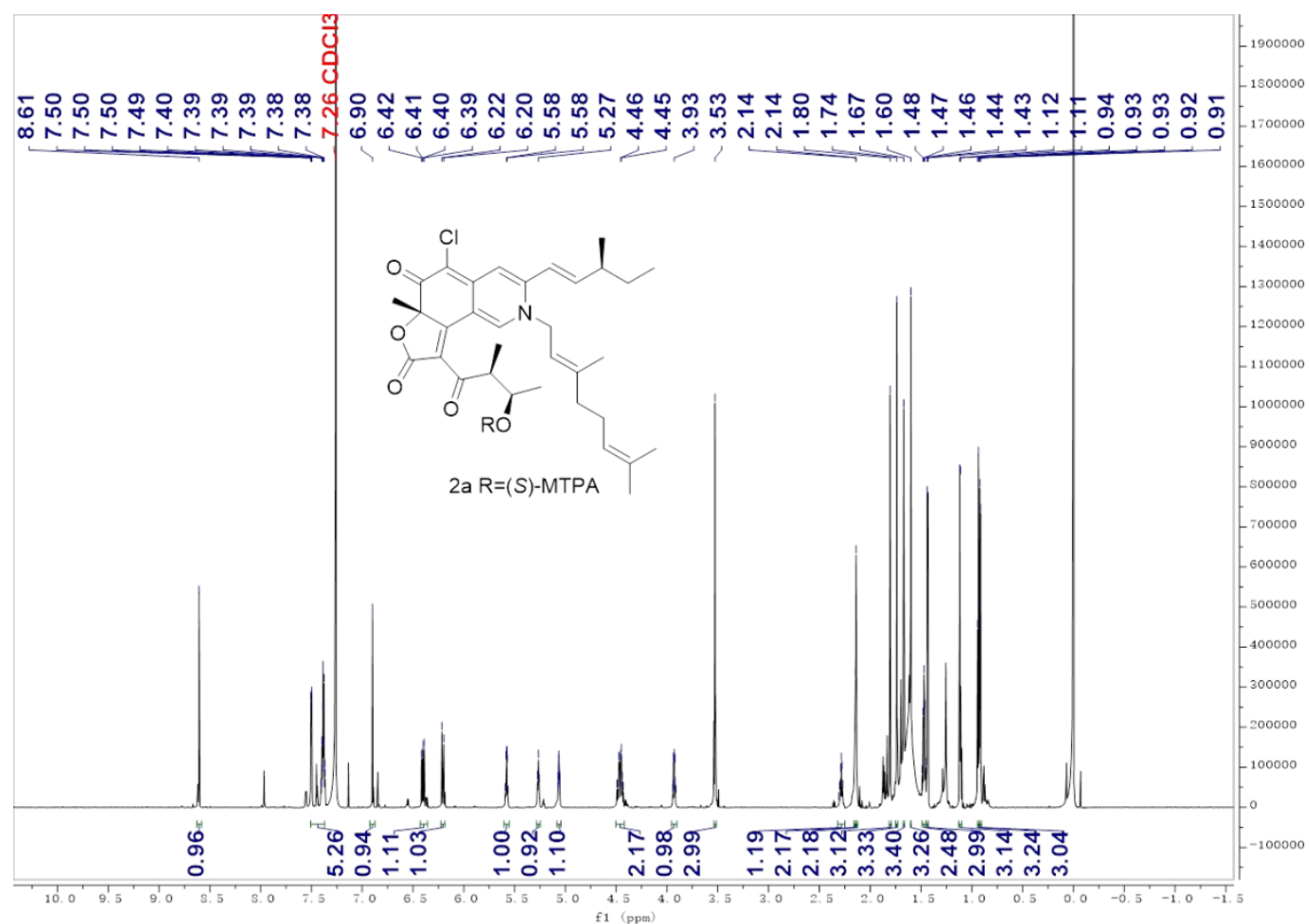

Figure S105. ${ }^{1} \mathrm{H}$ NMR spectrum of $(S)$-MTPA ester of 2 (2a)

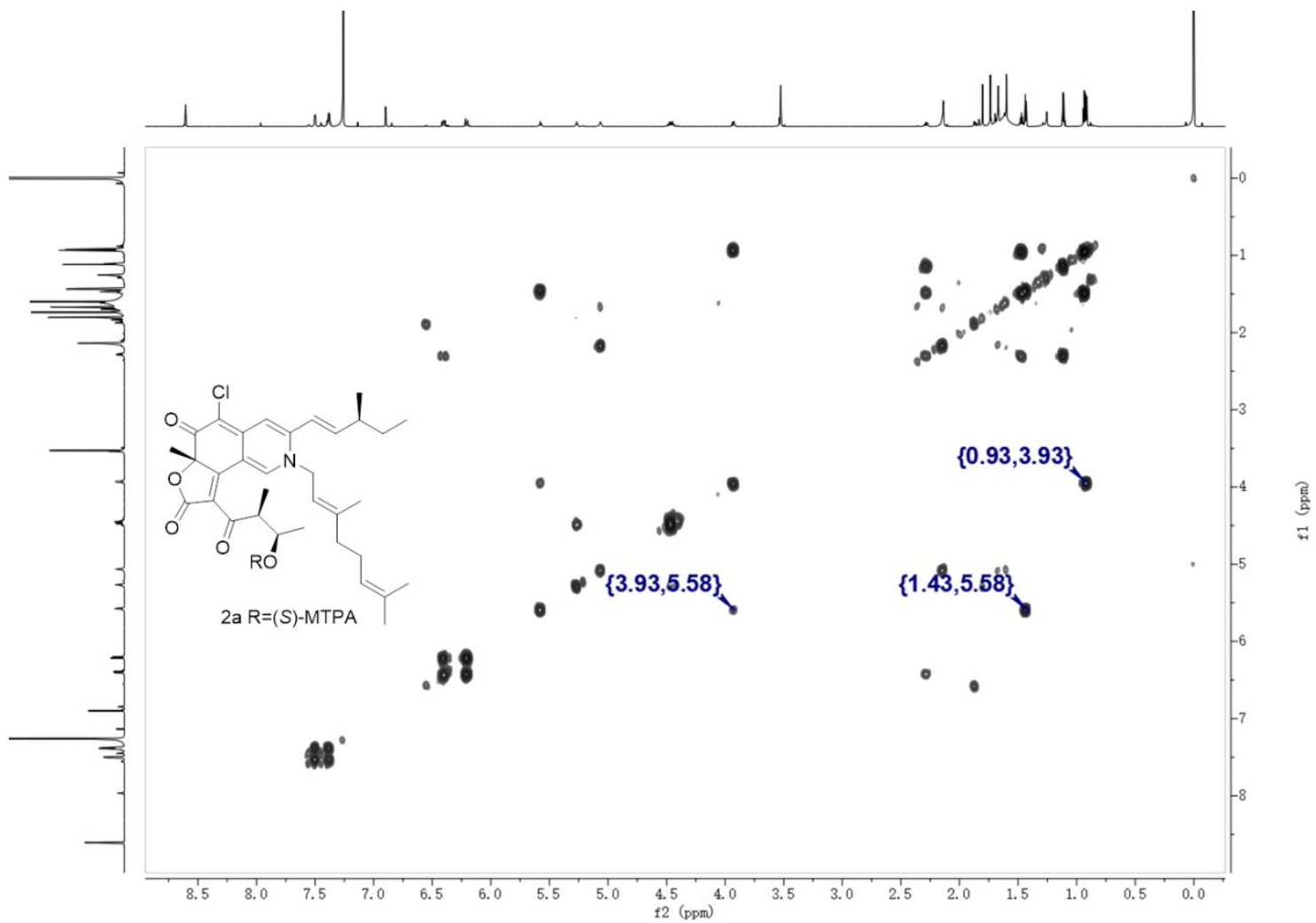

Figure S106. COSY spectrum of (S)-MTPA ester of 1 (2a) 


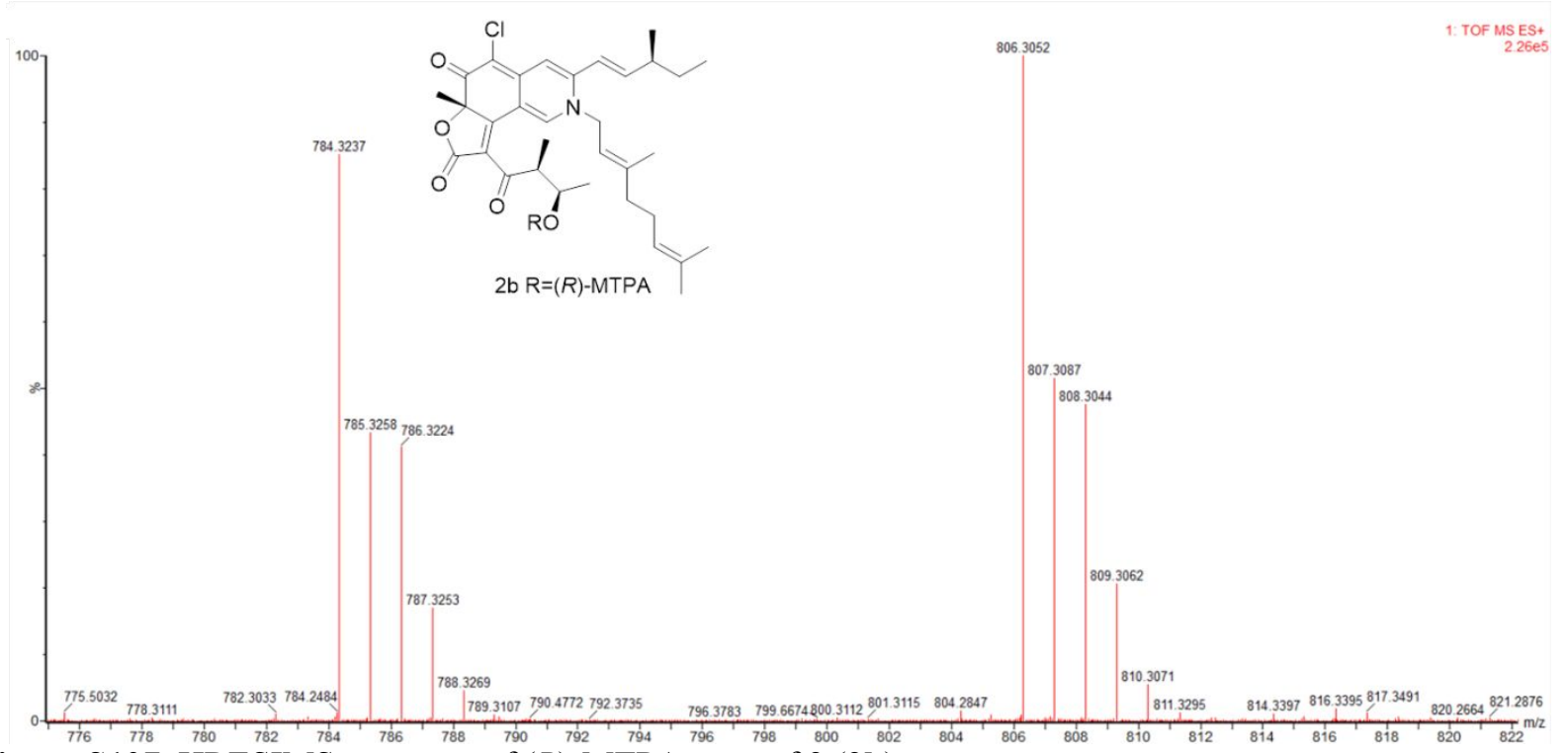

Figure S107. HRESIMS spectrum of (R)-MTPA ester of 2 (2b)

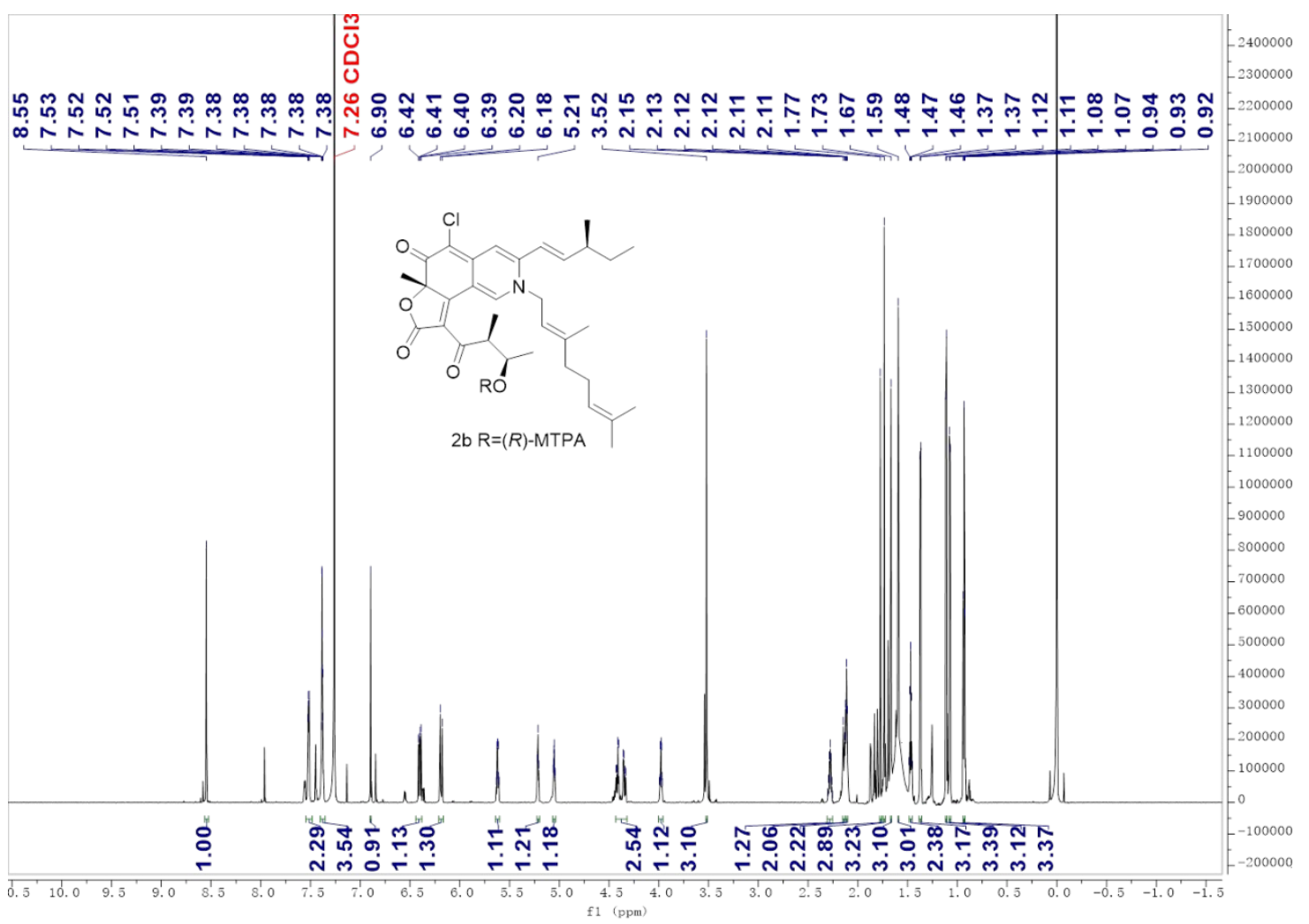

Figure S108. ${ }^{1} \mathrm{H}$ NMR spectrum of $(R)$-MTPA ester of $\mathbf{2}$ (2b) 


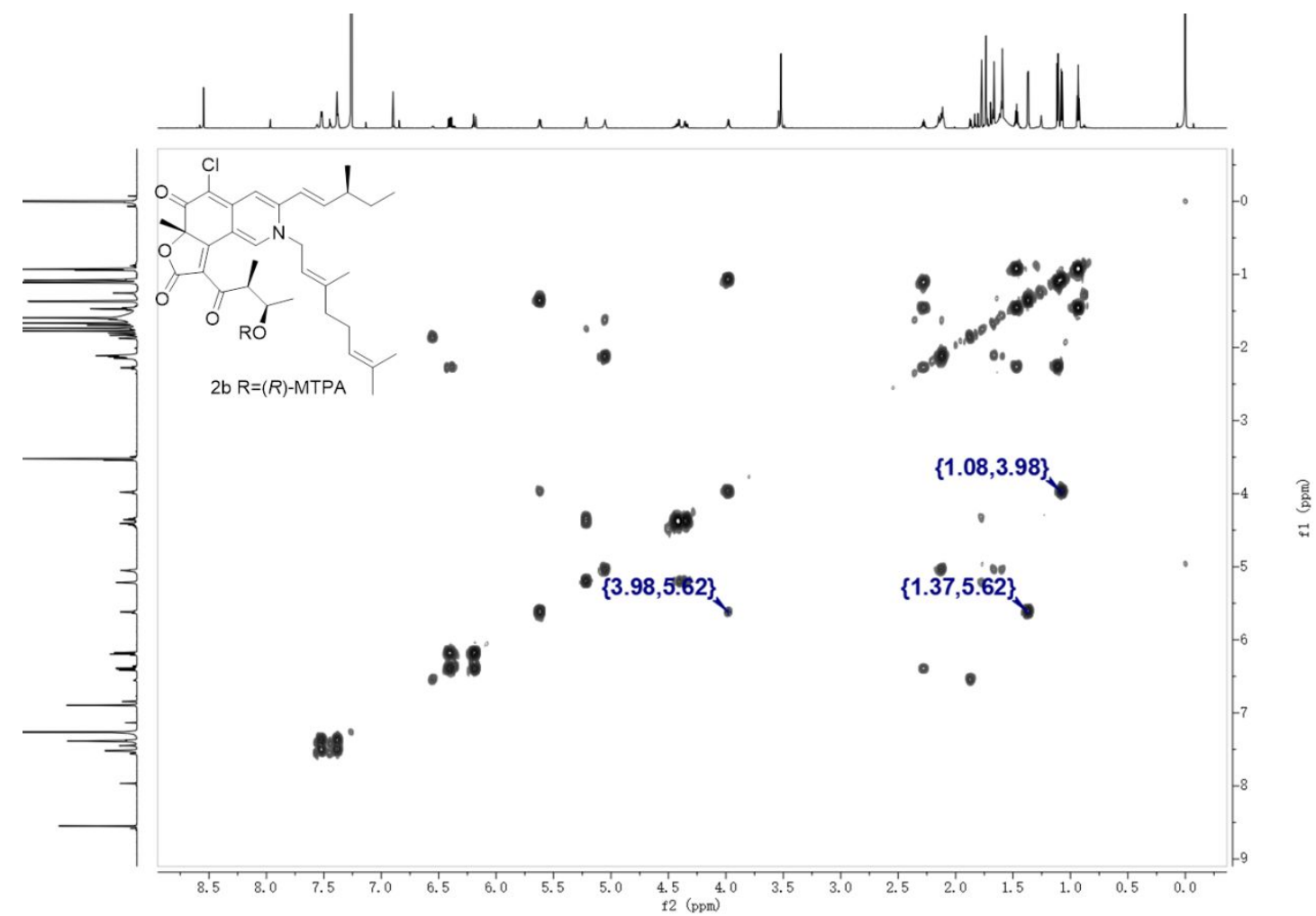

Figure S109. COSY spectrum of (S)-MTPA ester of 2 (2b)

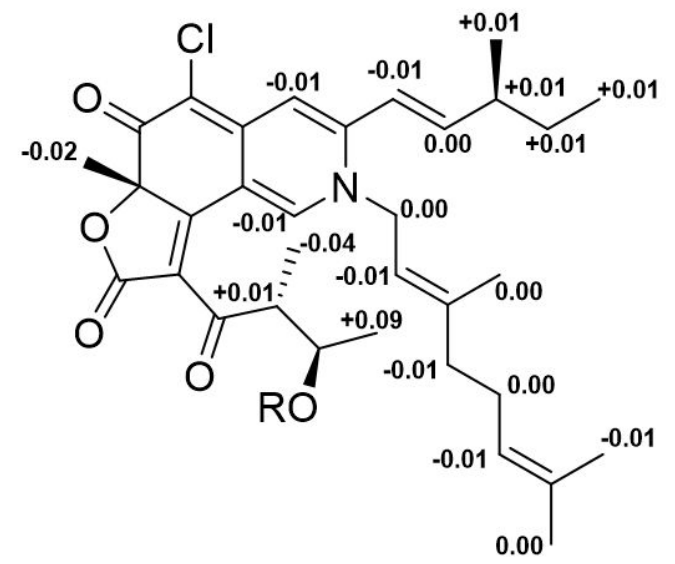

1a $\mathrm{R}=(S)-M T P A$ $1 \mathrm{~b} \mathrm{R}=(R)-\mathrm{MTPA}$

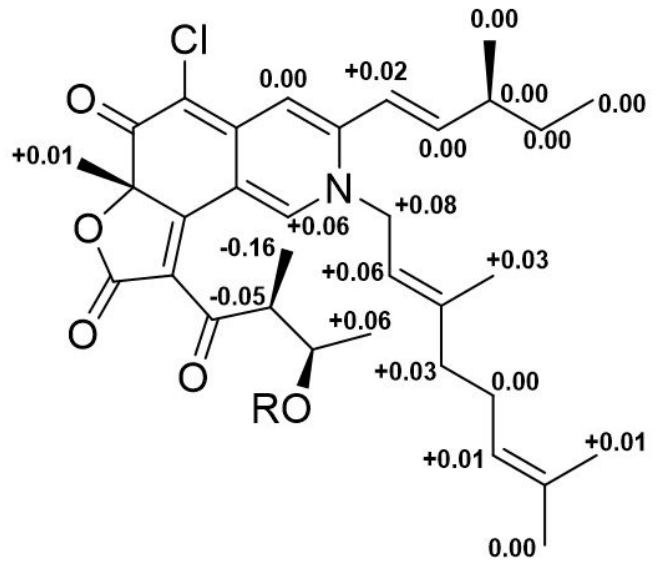

2a $\mathrm{R}=(S)-M T P A$ $2 \mathrm{~b} \mathrm{R}=(R)-\mathrm{MTPA}$

Figure S110. $\Delta \delta_{S-R}$ values for the MTPA derivatives of 1-2 in chloroform- $d$ 


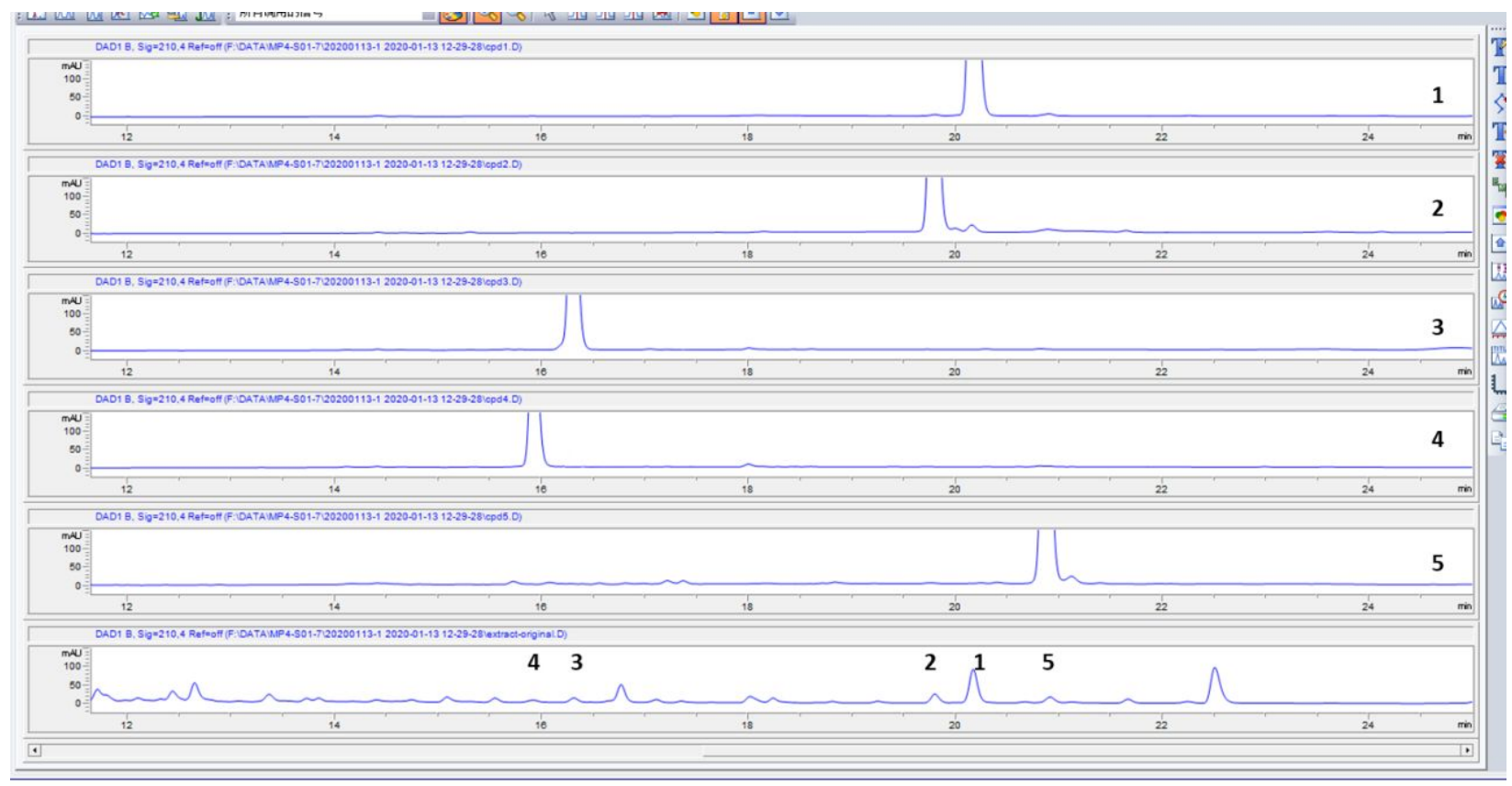

Figure S11. HPLC spectra of extract and compounds 1-5

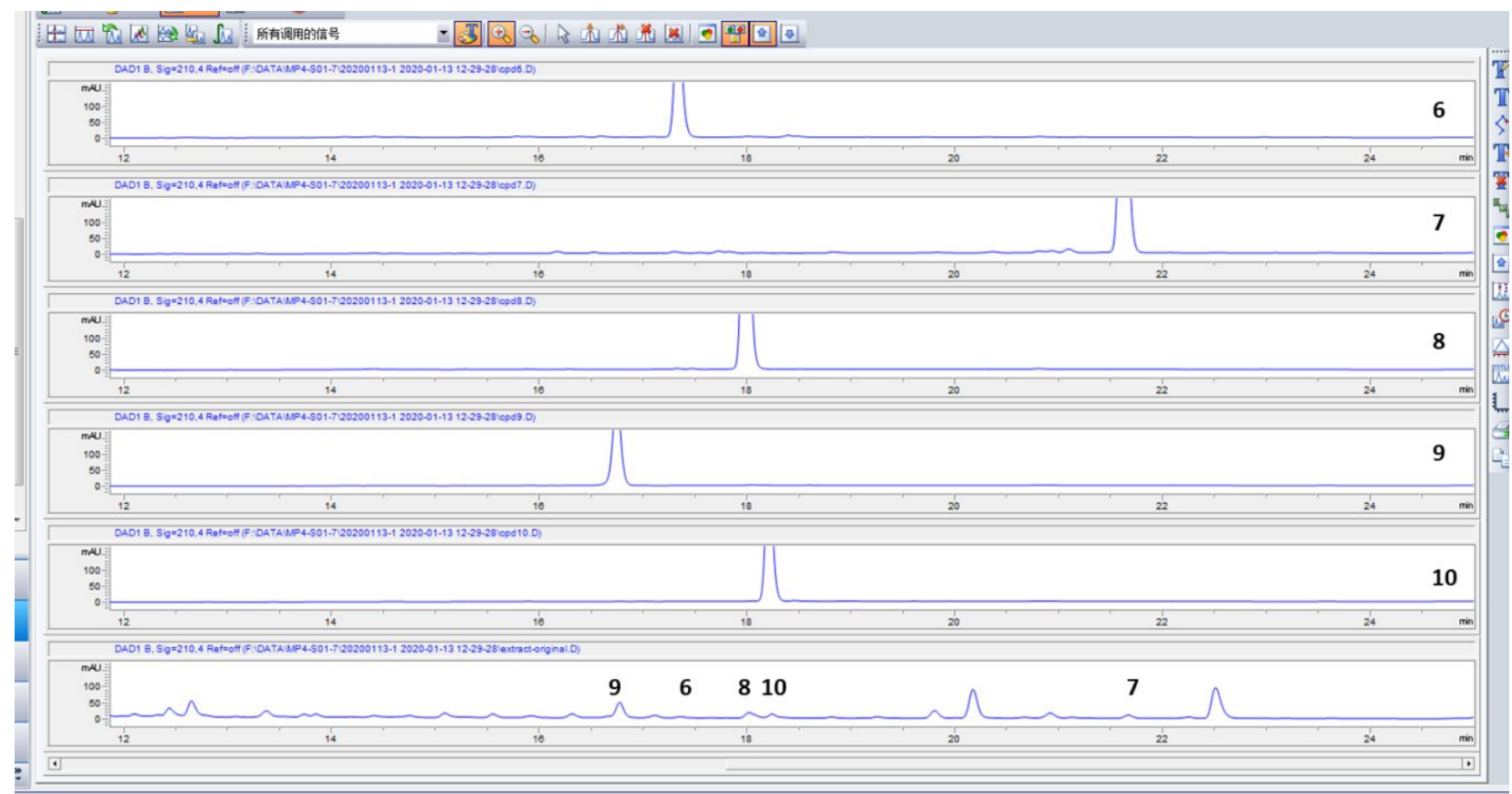

Figure S112. HPLC spectra of extract and compounds 5-10 


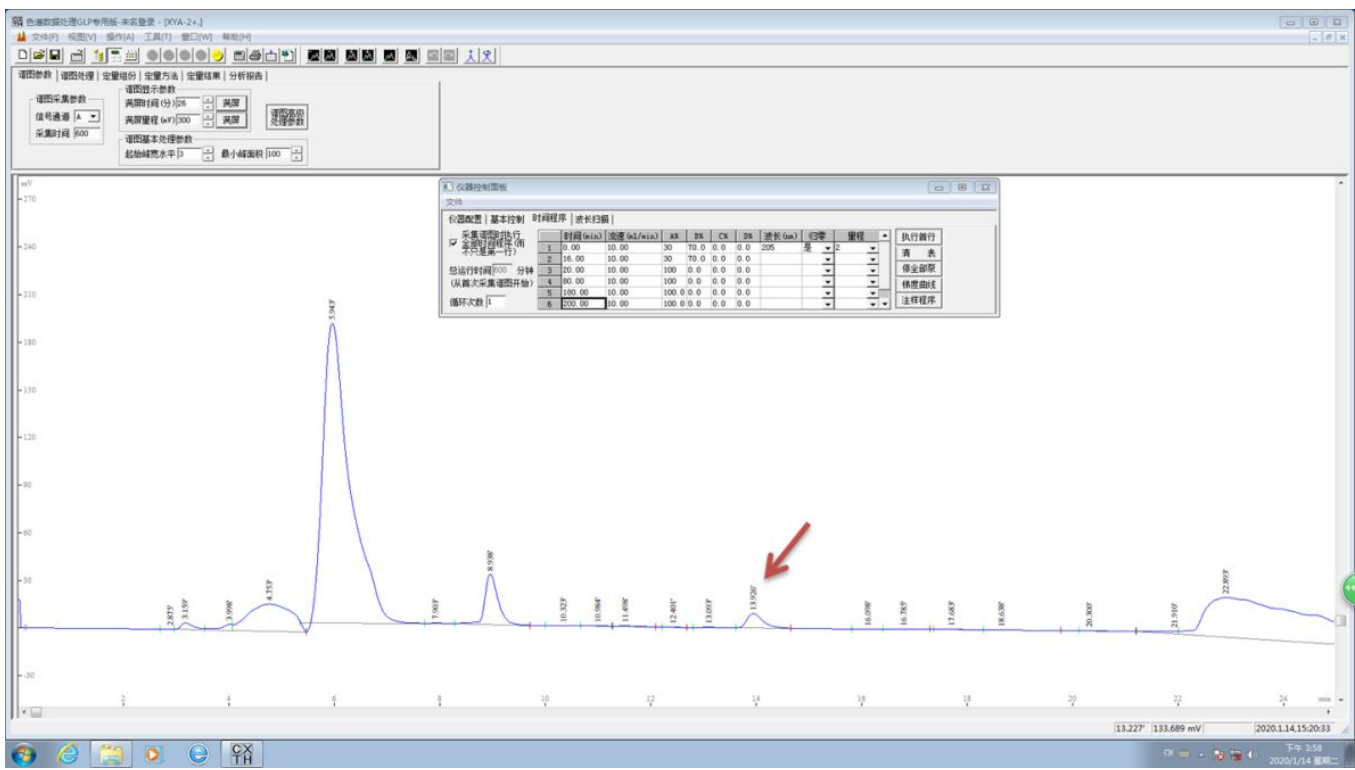

Figure S113. Preparative HPLC spectrum of $(S)$-2-methylbutyric acid from the reaction mixture of chromium trioxide oxidation of compound 1 